Supporting Information

\title{
Cascade Ring-Opening Dual Halogenation of Cyclopropenones with Saturated Oxygen Heterocycles
}

Wei-Hang Miao, Wen-Xia Gao, Xiao-Bo Huang, Miao-Chang Liu, * Yun-Bing Zhou, $*$ and Hua-Yue $\mathrm{Wu}$

College of Chemistry and Materials Engineering, Wenzhou University, Wenzhou, 325035, P. R. of China

\section{Content}

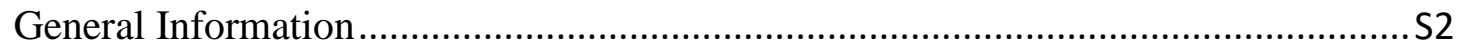

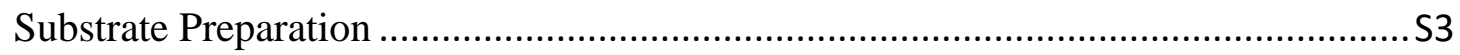

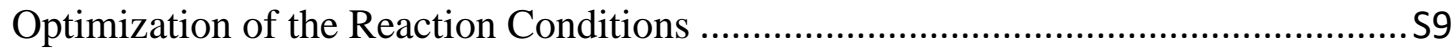

General Experimental Procedures............................................................... S11

Characterization of Products in Details ...................................................................... S13

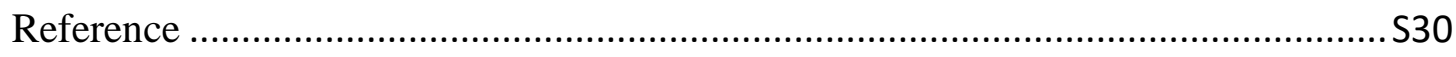

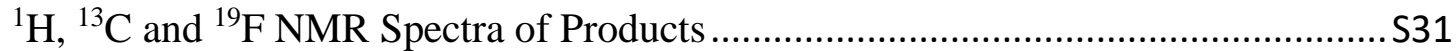




\section{General Information}

All reagents and solvents were purchased from TCI, Sigma-Aldrich, Alfa Aesar, Acros and Meryer. All reactions were conducted using standard Schlenk techniques. Column chromatography was performed using EM silica gel 60 (300-400 mesh). ${ }^{1} \mathrm{H}$ NMR and ${ }^{13} \mathrm{C}$ NMR spectra were measured on a $500 \mathrm{MHz}$ Bruker AVANCE spectrometer (500 $\mathrm{MHz}$ for ${ }^{1} \mathrm{H}, 125 \mathrm{MHz}$ for ${ }^{13} \mathrm{C}, 470 \mathrm{MHz}$ for ${ }^{19} \mathrm{~F}$ ), using DMSO-d6 or $\mathrm{CDCl}_{3}$ as the solvent with tetramethylsilane (TMS) as the internal standard at room temperature. Chemical shifts were reported in ppm. ${ }^{1} \mathrm{H}$ NMR spectra were referenced to $\mathrm{CDCl}_{3}(7.26$ $\mathrm{ppm})$ or DMSO- $d_{6}(2.50 \mathrm{ppm})$, and ${ }^{13} \mathrm{C}-\mathrm{NMR}$ spectra were referenced to $\mathrm{CDCl}_{3}(77.0$ $\mathrm{ppm})$ or DMSO-d$(39.5 \mathrm{ppm})$ were referenced to $\mathrm{CDCl}_{3}$, Peak multiplicities were designated by the following abbreviations: s, singlet; $d$, doublet; t, triplet; m, multiplet. Chemical shifts are given in $\delta$ relative to TMS, the coupling constants $J$ are given in Hz. Analysis of crude reaction mixture was done on the Varian 4000 GC/MS and Agilent 7890A/5975C. High-resolution mass spectra were recorded on a micrOTOF-Q II 10410 mass spectrometer. Saturated oxygen heterocycles for substrates (2) are commercially available. Unless otherwise noted, all reagents and solvents were obtained commercially and used without further purification. 


\section{Substrate Preparation}

(1) General procedure for the synthesis of cyclopropenones $\left(1,3-9\right.$, Method I) ${ }^{1}$<smiles>O=C(O)Cc1cc[R]cc1</smiles>

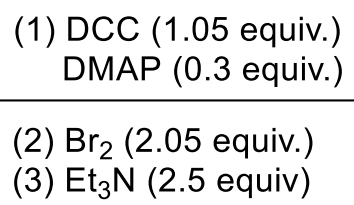

(2) $\mathrm{Br}_{2}$ (2.05 equiv.)

(3) $\mathrm{Et}_{3} \mathrm{~N}$ (2.5 equiv)<smiles>[R][R]1ccc(C2C(=O)C2c2cc#[R]cc2)cc1</smiles>

Substituted 2,3-diphenylcycloprop-2-enones were prepared in three steps from substituted phenylacetic acid. The diaryl ketone was prepared by adding substituted phenylacetic acid (20mmol, 1 equiv) to a stirring solution of DCC (1.05 equiv) and DMAP (0.3 equiv) in THF at room temperature. After $24 \mathrm{~h}$, the reaction was filtered through Celite. The diaryl ketone was purified by flash chromatography on silica gel. The diaryl ketone was then dissolved in acetic acid, and a solution of bromine (2.05 equiv) in acetic acid was added dropwise. After 3 hours, the reaction was poured into water and the product was collected by filtration. The crude product (dibromoketone) was air dried for several hours, then dissolved in dry dichloromethane. This solution was added to a stirring solution of triethylamine (2.5 equiv) in dichloromethane, and stirred at room temperature for 1 hour. The reaction mixture was then washed with $1 \mathrm{M}$ $\mathrm{HCl}$, followed by brine, dried with sodium sulfate, and concentrated under reduced pressure. The product was purified by column chromatography on silica gel.

\section{2,3-diphenylcycloprop-2-en-1-one (1) ${ }^{2}$}<smiles>O=c1c(-c2ccccc2)c1-c1ccccc1</smiles>

The general procedure (Method I) was followed, and 3/1 hexane/ethyl acetate was used as the eluant to afford white solid $(1.39 \mathrm{~g}, 67 \%)$, Melting Point: $119-120{ }^{\circ} \mathrm{C} ;{ }^{1} \mathrm{H}$ NMR (500 MHz, Chloroform- $d$ ) $\delta 8.0-7.97(\mathrm{~m}, 4 \mathrm{H}), 7.62-7.56(\mathrm{~m}, 6 \mathrm{H}) .{ }^{13} \mathrm{C}$ NMR $(125$ MHz, Chloroform- $d$ ) $\delta 155.9,148.3,132.7,131.5,129.4,124.0$.

\section{2,3-bis(4-bromophenyl)cycloprop-2-en-1-one $(3)^{3}$}


<smiles>O=c1c(-c2ccc(Br)cc2)c1-c1ccc(Br)cc1</smiles>

The general procedure (Method I) was followed, and 3/1 hexane/ethyl acetate was used as the eluant to afford white solid $(2.15 \mathrm{~g}, 59 \%)$, Melting Point: $263-264{ }^{\circ} \mathrm{C} ;{ }^{1} \mathrm{H}$ NMR $\left(500 \mathrm{MHz}\right.$, Chloroform- $d$ ) $\delta 7.81(\mathrm{~d}, J=8.0 \mathrm{~Hz}, 4 \mathrm{H}), 7.74(\mathrm{~d}, J=8.0 \mathrm{~Hz}, 4 \mathrm{H}) .{ }^{13} \mathrm{C} \mathrm{NMR}$ $(125 \mathrm{MHz}$, Chloroform- $d$ ) $\delta$ 155.0, 147.6, 132.9, 132.7, 128.0, 122.6.

\section{2,3-bis(4-fluorophenyl)cycloprop-2-en-1-one (4) ${ }^{1}$}<smiles>O=c1c(-c2ccc(F)cc2)c1-c1ccc(F)cc1</smiles>

The general procedure (Method I) was followed, and 3/1 hexane/ethyl acetate was used as the eluant to afford white solid $(1.58 \mathrm{~g}, 65 \%)$, Melting Point: $184-185{ }^{\circ} \mathrm{C}$; ${ }^{1} \mathrm{H}$ NMR $\left(500 \mathrm{MHz}\right.$, Chloroform- $d$ ) $\delta 7.99(\mathrm{dd}, J=6.8,2.3 \mathrm{~Hz}, 4 \mathrm{H}), 7.62-7.56(\mathrm{~m}, 6 \mathrm{H}) .{ }^{13} \mathrm{C}$ NMR (125 MHz, Chloroform- $d$ ) $\delta 165.0(J=255 \mathrm{~Hz}), 154.9,146.2,133.8(J=9.25$ $\mathrm{Hz}), 120.4(J=3.25 \mathrm{~Hz}), 116.9(J=22 \mathrm{~Hz})$.

\section{2,3-bis(4-chlorophenyl)cycloprop-2-en-1-one (5) ${ }^{1}$}<smiles>O=c1c(-c2ccc(Cl)cc2)c1-c1ccc(Cl)cc1</smiles>

The general procedure (Method I) was followed, and 3/1 hexane/ethyl acetate was used as the eluant to afford white solid (1.59 g, 58\%), Melting Point: $204-205{ }^{\circ} \mathrm{C} ;{ }^{1} \mathrm{H}$ NMR $\left(500 \mathrm{MHz}\right.$, Chloroform- $d$ ) $\delta 7.89(\mathrm{~d}, J=8.2 \mathrm{~Hz}, 4 \mathrm{H}), 7.57(\mathrm{~d}, J=8.2 \mathrm{~Hz}, 4 \mathrm{H}) .{ }^{13} \mathrm{C} \mathrm{NMR}$ (125 MHz, Chloroform- $d$ ) $\delta$ 155.0, 147.3, 139.3, 132.8, 132.6, 129.9, 128.7, 122.2.

\section{2,3-di-p-tolylcycloprop-2-en-1-one (6) ${ }^{1}$}<smiles>Cc1ccc(-c2c(-c3ccc(C)cc3)c2=O)cc1</smiles>

The general procedure (Method I) was followed, and 3/1 hexane/ethyl acetate was used as the eluant to afford white solid $(1.39 \mathrm{~g}, 59 \%)$, Melting Point: $166-167{ }^{\circ} \mathrm{C} ;{ }^{1} \mathrm{H}$ NMR $(500 \mathrm{MHz}$, Chloroform- $d$ ) $\delta 7.85(\mathrm{~d}, J=7.8 \mathrm{~Hz}, 4 \mathrm{H}), 7.36(\mathrm{~d}, J=7.8 \mathrm{~Hz}, 4 \mathrm{H}), 2.45$ (s, $6 \mathrm{H}) .{ }^{13} \mathrm{C}$ NMR (125 MHz, Chloroform- $d$ ) $\delta$ 155.8, 146.6, 143.5, 131.5, 130.0, 121.4, 21.8 . 


\section{2,3-bis(4-(tert-butyl)phenyl)cycloprop-2-en-1-one (7)}<smiles>CC(C)(C)c1ccc(-c2c(-c3ccc(C(C)(C)C)cc3)c2=O)cc1</smiles>

The general procedure (Method I) was followed, and 3/1 hexane/ethyl acetate was used as the eluant to afford white solid $(1.57 \mathrm{~g}, 49 \%)$, Melting Point: $239-240{ }^{\circ} \mathrm{C} ;{ }^{1} \mathrm{H}$ NMR $(500 \mathrm{MHz}$, Chloroform- $d$ ) $\delta 7.93(\mathrm{~d}, J=8.2 \mathrm{~Hz}, 4 \mathrm{H}), 7.59(\mathrm{~d}, J=8.2 \mathrm{~Hz}, 4 \mathrm{H}), 1.38(\mathrm{~s}$, $18 \mathrm{H}) .{ }^{13} \mathrm{C}$ NMR (125 MHz, Chloroform- $d$ ) $\delta$ 156.4, 155.8, 146.8, 131.4, 126.3, 121.4, $35.3,31.1$.

2,3-bis(3-bromophenyl)cycloprop-2-en-1-one $(8)^{4}$<smiles>O=c1c(-c2cccc(Br)c2)c1-c1cccc(Br)c1</smiles>

The general procedure (Method I) was followed, and 3/1 hexane/ethyl acetate was used as the eluant to afford white solid (2.08 g, 57\%), Melting Point: $247-248{ }^{\circ} \mathrm{C} ;{ }^{1} \mathrm{H}$ NMR $(500 \mathrm{MHz}$, Chloroform- $d$ ) $\delta 8.10-8.04(\mathrm{~m}, 2 \mathrm{H}), 7.88(\mathrm{~d}, J=7.7 \mathrm{~Hz}, 2 \mathrm{H}), 7.77-7.71$ (m, 2H), 7.49 (t, $J=7.9 \mathrm{~Hz}, 2 \mathrm{H}) .{ }^{13} \mathrm{C}$ NMR (125 MHz, Chloroform- $d$ ) $\delta$ 154.8, 148.4, $135.9,134.1,131.0,129.8,125.3,123.4$.

\section{2,3-bis(3-chlorophenyl)cycloprop-2-en-1-one (9)}<smiles>O=c1c(-c2cccc(Cl)c2)c1-c1cccc(Cl)c1</smiles>

The general procedure (Method I) was followed, and 3/1 hexane/ethyl acetate was used as the eluant to afford white solid $(1.60 \mathrm{~g}, 58 \%)$, Melting Point: $188-189{ }^{\circ} \mathrm{C} ;{ }^{1} \mathrm{H}$ NMR $(500 \mathrm{MHz}$, Chloroform- $d$ ) $\delta 7.93$ (t, $J=1.9 \mathrm{~Hz}, 2 \mathrm{H}), 7.85$ (d, $J=7.4 \mathrm{~Hz}, 2 \mathrm{H}), 7.61-$ $7.58(\mathrm{~m}, 2 \mathrm{H}), 7.55$ (t, $J=7.8 \mathrm{~Hz}, 2 \mathrm{H}) .{ }^{13} \mathrm{C}$ NMR (125 MHz, Chloroform- $d$ ) $\delta$ 154.9, 148.5, 135.5, 133.0, 131.2, 130.8, 129.4, 125.1. HRMS: (ESI) calculated for $\mathrm{C}_{15} \mathrm{H}_{9} \mathrm{Cl}_{2} \mathrm{O}$ $[\mathrm{M}+\mathrm{H}]^{+}$275.0025, found 275.0029 .

(2) General procedure for the synthesis of cyclopropenones (10-17, Method II) ${ }^{5}$ 


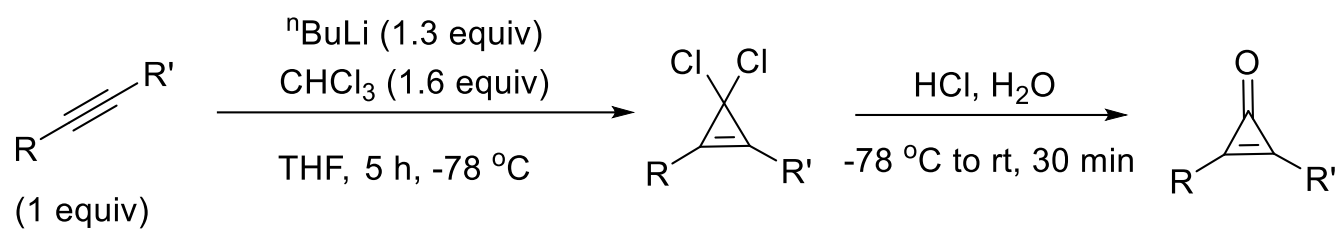

A three-necked $250 \mathrm{~mL}$ flask with a stir bar was equipped with dropping funnel, an outlet tube connected to the nitrogen/vacuum-line and a septum. Then the apparatus was evacuated in high vacuum, heated with a heat gun and backfilled with nitrogen for three times in order to exclude air and moisture. Subsequently, the reaction flask was charged with substituted alkyne (50 mmol, 1.0 equiv), chloroform (reagent-grade, 6.45 $\mathrm{mL}, 9.55 \mathrm{~g}, 80 \mathrm{mmol}, 1.6$ equiv) and dry THF (50 mL, $1 \mathrm{M})$ and the septa was exchanged by a quick-fit with thermometer. The resulting clear reaction solution was cooled down to $-78{ }^{\circ} \mathrm{C}$ and a $1.6 \mathrm{M}^{\mathrm{n}} \mathrm{BuLi}$ solution in hexane $(40.6 \mathrm{~mL}, 65.0 \mathrm{mmol}, 1.3$ equiv) was added dropwise over $1 \mathrm{~h}$ by means of the dropping funnel under stirring. Thereby, the reaction mixture turned black after the addition of a couple of drops of the ${ }^{\mathrm{n}} \mathrm{BuLi}$ solution, while the internal temperature stayed below $-70{ }^{\circ} \mathrm{C}$. In the following, the dropping funnel was rinsed with dry THF $(2 \mathrm{~mL})$ and the reaction mixture was stirred for $4 \mathrm{~h}$ at $-78{ }^{\circ} \mathrm{C}$. Next, aqueous $\mathrm{HCl}$ solution $(37 \mathrm{wt} \%, 25 \mathrm{~mL}$ ) was added dropwise with the aid of the dropping funnel over 30 min under vigorous stirring at $78{ }^{\circ} \mathrm{C}$. Then $\mathrm{CH}_{2} \mathrm{Cl}_{2}(50 \mathrm{~mL})$ was added by means of the dropping funnel within $5 \mathrm{~min}$, after 15 min of stirring the cooling bath was removed and the mixture was allowed to stir for $30 \mathrm{~min}$ at ambient temperature. Subsequently, the mixture was diluted with water $(150 \mathrm{~mL})$ to improve the phase separation, transferred to an extraction funnel, the reaction flask was rinsed with further $\mathrm{CH}_{2} \mathrm{Cl}_{2}(3 \times 10 \mathrm{~mL})$, the phases were separated and the aqueous phase was extracted with $\mathrm{CH}_{2} \mathrm{Cl}_{2}(3 \times 100 \mathrm{~mL})$. The combined organic phases were dried over $\mathrm{MgSO}_{4}$, concentrated under reduced pressure and purified by column chromatography on silica gel.

\section{2-(4-methoxyphenyl)cycloprop-2-en-1-one (10) ${ }^{6}$}

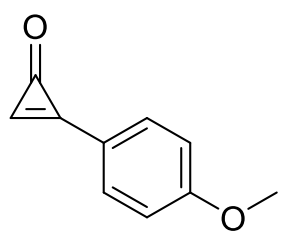


The general procedure (Method II) was followed, and 3/1 hexane/ethyl acetate was used as the eluant to afford brown oil $(3.25 \mathrm{~g}, 40 \%),{ }^{1} \mathrm{H}$ NMR $(500 \mathrm{MHz}$, Chloroform- $d$ ) $\delta$ $8.28(\mathrm{~s}, 1 \mathrm{H}), 7.84-7.80(\mathrm{~m}, 2 \mathrm{H}), 7.06-7.02(\mathrm{~m}, 2 \mathrm{H}), 3.90(\mathrm{~s}, 3 \mathrm{H}) .{ }^{13} \mathrm{C}$ NMR $(125$ $\mathrm{MHz}$, Chloroform- $d$ ) $\delta 163.7,160.8,155.2,136.5,133.5,116.0,114.7,55.6$.

\section{2-(4-bromophenyl)cycloprop-2-en-1-one (11)}<smiles>O=c1cc1-c1ccc(Br)cc1</smiles>

The general procedure (Method II) was followed, and 3/1 hexane/ethyl acetate was used as the eluant to afford brown oil (4.75 g, 45\%), ${ }^{1} \mathrm{H}$ NMR (500 MHz, Chloroform- $d$ ) $\delta$ $8.56(\mathrm{~s}, 1 \mathrm{H}), 7.72(\mathrm{~d}, J=1.8 \mathrm{~Hz}, 4 \mathrm{H}) .{ }^{13} \mathrm{C}$ NMR $(125 \mathrm{MHz}$, Chloroform- $d$ ) $\delta$ 161.0, 154.7, 141.5, 132.8, 132.4 128.8, 121.9. HRMS: (ESI) calculated for $\mathrm{C}_{9} \mathrm{H}_{6} \mathrm{BrO}[\mathrm{M}+\mathrm{H}]^{+}$ 208.9597, found 208.9595.

\section{2-(naphthalen-2-yl)cycloprop-2-en-1-one (12)}<smiles>O=c1cc1-c1ccc2ccccc2c1</smiles>

The general procedure (Method II) was followed, and 3/1 hexane/ethyl acetate was used as the eluant to afford brown oil (4.1 g, 45\%), ${ }^{1} \mathrm{H}$ NMR $(500 \mathrm{MHz}$, Chloroform- $d$ ) $\delta$ $8.53(\mathrm{~s}, 1 \mathrm{H}), 8.38(\mathrm{~s}, 1 \mathrm{H}), 7.92(\mathrm{t}, J=7.3 \mathrm{~Hz}, 2 \mathrm{H}), 7.86(\mathrm{~d}, J=8.1 \mathrm{~Hz}, 1 \mathrm{H}), 7.77(\mathrm{~d}, J$ $=8.5 \mathrm{~Hz}, 1 \mathrm{H}), 7.61(\mathrm{t}, J=7.5 \mathrm{~Hz}, 1 \mathrm{H}), 7.56(\mathrm{t}, J=7.6 \mathrm{~Hz}, 1 \mathrm{H}) .{ }^{13} \mathrm{C} \mathrm{NMR}(125 \mathrm{MHz}$, Chloroform- $d$ ) $\delta 161.8,155.5,140.6,135.3,133.8,132.7,129.3,129.1,128.0,127.3$, 125.4, 120.1. HRMS: (ESI) calculated for $\mathrm{C}_{13} \mathrm{H}_{9} \mathrm{O}[\mathrm{M}+\mathrm{H}]^{+}$181.0648, found 181.0649.

\section{2-methyl-3-phenylcycloprop-2-en-1-one $(13)^{7}$}<smiles>Cc1c(-c2ccccc2)c1=O</smiles>

The general procedure (Method II) was followed, and 3/1 hexane/ethyl acetate was used as the eluant to afford orange solid $(3.05 \mathrm{~g}, 42 \%)$, Melting Point: $72-73{ }^{\circ} \mathrm{C} ;{ }^{1} \mathrm{H}$ NMR $\left(500 \mathrm{MHz}\right.$, Chloroform- $d$ ) $\delta 7.81-7.75(\mathrm{~m}, 2 \mathrm{H}), 7.58-7.50(\mathrm{~m}, 3 \mathrm{H}), 2.49(\mathrm{~s}, 3 \mathrm{H}) .{ }^{13} \mathrm{C}$ NMR (125 MHz, Chloroform- $d$ ) $\delta$ 156.8, 154.7, 151.6, 132.5, 131.0, 129.2, 123.7, 11.5 .

\section{2-isopropyl-3-phenylcycloprop-2-en-1-one $(14)^{8}$}


<smiles>CC(C)c1c(-c2ccccc2)c1=O</smiles>

The general procedure (Method II) was followed, and 3/1 hexane/ethyl acetate was used as the eluant to afford brown oil $(3.55 \mathrm{~g}, 41 \%),{ }^{1} \mathrm{H}$ NMR $(500 \mathrm{MHz}$, Chloroform- $d) \delta$ $7.82-7.77(\mathrm{~m}, 2 \mathrm{H}), 7.58-7.50(\mathrm{~m}, 3 \mathrm{H}), 3.23-3.14(\mathrm{~m}, 1 \mathrm{H}), 1.43(\mathrm{~d}, J=7.2 \mathrm{~Hz}, 6 \mathrm{H})$. ${ }^{13} \mathrm{C}$ NMR (125 MHz, Chloroform- $d$ ) $\delta 159.4,157.4,153.0,132.4,131.4,129.2,123.4$, 27.6, 20.2, 20.2.

\section{(3-oxo-2-phenylcycloprop-1-en-1-yl)methyl acetate (15) ${ }^{9}$}<smiles>CC(=O)OCc1c(-c2ccccc2)c1=O</smiles>

The general procedure (Method II) was followed, and 3/1 hexane/ethyl acetate was used as the eluant to afford brown oil $(5.19 \mathrm{~g}, 51 \%),{ }^{1} \mathrm{H}$ NMR $(500 \mathrm{MHz}$, Chloroform- $d) \delta$ $7.84(\mathrm{~d}, J=7.6 \mathrm{~Hz}, 2 \mathrm{H}), 7.60$ (t, $J=7.4 \mathrm{~Hz}, 1 \mathrm{H}), 7.54(\mathrm{t}, J=7.4 \mathrm{~Hz}, 2 \mathrm{H}), 5.30(\mathrm{~s}, 2 \mathrm{H})$, $2.25(\mathrm{~s}, 3 \mathrm{H}) .{ }^{13} \mathrm{C}$ NMR $(125 \mathrm{MHz}$, Chloroform- $d$ ) $\delta 169.9,153.9,149.7,133.1,131.7$, $129.3,122.8,59.3,20.6$.

\section{2,3-diethylcycloprop-2-en-1-one (16) ${ }^{10}$}<smiles>CCc1c(CC)c1=O</smiles>

The general procedure (Method II) was followed, and 3/1 hexane/ethyl acetate was used as the eluant to afford brown oil $(2.45 \mathrm{~g}, 44 \%),{ }^{1} \mathrm{H}$ NMR $(500 \mathrm{MHz}$, Chloroform- $d$ ) $\delta$ $2.64(\mathrm{q}, J=7.5 \mathrm{~Hz}, 2 \mathrm{H}), 1.31(\mathrm{t}, J=7.6 \mathrm{~Hz}, 3 \mathrm{H}) .{ }^{13} \mathrm{C}$ NMR $(125 \mathrm{MHz}$, Chloroform- $d$ ) $\delta 161.3,159.7,19.7,11.0$.

\section{2,3-dibutylcycloprop-2-en-1-one (17) $)^{11}$}

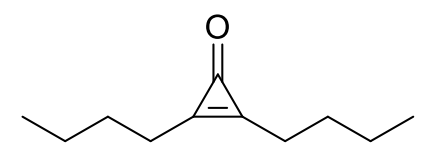

The general procedure (Method II) was followed, and 3/1 hexane/ethyl acetate was used as the eluant to afford brown oil $(4.09 \mathrm{~g}, 49 \%),{ }^{1} \mathrm{H}$ NMR $(400 \mathrm{MHz}$, Chloroform- $d$ ) $\delta$ $2.60(\mathrm{t}, J=7.3 \mathrm{~Hz}, 4 \mathrm{H}), 1.67(\mathrm{p}, J=7.3 \mathrm{~Hz}, 4 \mathrm{H}), 1.47-1.37(\mathrm{~m}, 4 \mathrm{H}), 0.95(\mathrm{t}, J=7.4$ $\mathrm{Hz}, 6 \mathrm{H}) .{ }^{13} \mathrm{C}$ NMR $(125 \mathrm{MHz}$, Chloroform- $d$ ) $\delta 160.7,160.0,28.2,25.9,22.2,13.5$. 


\section{Optimization of the Reaction Conditions}

Table S1. Reaction Optimization for Ring-Opening Chlorination ${ }^{[a]}$

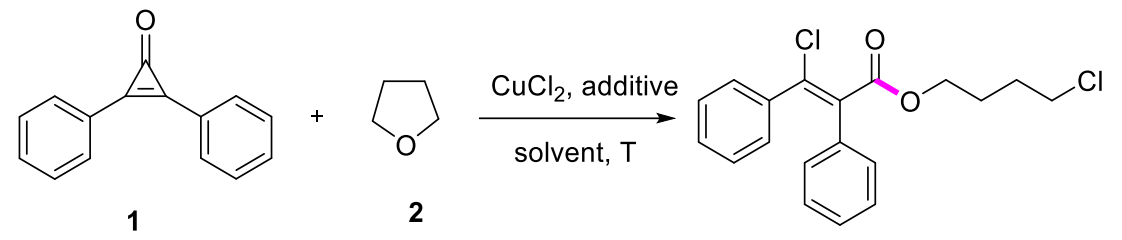

\begin{tabular}{|c|c|c|c|c|c|}
\hline entry & {$[\mathrm{Cu}]$} & additive & solvent & $\mathrm{T}\left({ }^{\circ} \mathrm{C}\right)$ & yield $(\%)$ \\
\hline 1 & $\mathrm{CuCl}_{2}$ & & $\mathrm{THF}$ & 120 & 24 \\
\hline 2 & $\mathrm{CuCl}_{2}$ & $\mathrm{AlCl}_{3}$ & THF & 120 & 75 \\
\hline 3 & $\mathrm{CuCl}_{2}$ & $\mathrm{AlCl}_{3}$ & THF & 100 & 68 \\
\hline 4 & $\mathrm{CuCl}_{2}$ & $\mathrm{AlCl}_{3}$ & THF & 80 & 48 \\
\hline 5 & $\mathrm{CuCl}_{2}$ & $\mathrm{AlCl}_{3}$ & $\mathrm{THF}$ & 60 & 43 \\
\hline 6 & $\mathrm{CuCl}_{2}$ & $\mathrm{AlCl}_{3}$ & THF & 40 & 0 \\
\hline 7 & $\mathrm{CuCl}$ & $\mathrm{AlCl}_{3}$ & $\mathrm{THF}$ & 120 & trace \\
\hline 8 & $\mathrm{CuCl}_{2}$ & $\mathrm{BF}_{3} \cdot \mathrm{Et}_{2} \mathrm{O}$ & THF & 120 & 21 \\
\hline 9 & $\mathrm{CuCl}_{2}$ & $\mathrm{NaCl}$ & THF & 120 & 24 \\
\hline 10 & & $\mathrm{AlCl}_{3}$ & THF & 120 & 0 \\
\hline 11 & $\mathrm{CuCl}_{2}$ & $\operatorname{Sc}(\mathrm{OTf})_{3}$ & THF & 120 & trace \\
\hline 12 & $\mathrm{CuCl}_{2}$ & AgOTf & THF & 120 & trace \\
\hline 13 & $\mathrm{CuCl}_{2}$ & $\mathrm{FeCl}_{3}$ & THF & 120 & trace \\
\hline 14 & $\mathrm{CuCl}_{2}$ & $\mathrm{FeCl}_{2}$ & THF & 120 & trace \\
\hline 15 & $\mathrm{CuCl}_{2}$ & $\mathrm{ZnCl}_{2}$ & THF & 120 & 40 \\
\hline 16 & $\mathrm{CuCl}_{2}$ & $\mathrm{NbCl}_{5}$ & THF & 120 & 55 \\
\hline 17 & $\mathrm{CuCl}_{2}$ & $\mathrm{CoCl}_{2}$ & THF & 120 & 47 \\
\hline 18 & $\mathrm{CuCl}_{2}$ & $\mathrm{SnCl}_{2}$ & THF & 120 & trace \\
\hline 19 & $\mathrm{CuCl}_{2}$ & $\mathrm{SnCl}_{4}$ & THF & 120 & trace \\
\hline 20 & $\mathrm{CuCl}_{2}$ & $\mathrm{TiCl}_{4}$ & $\mathrm{THF}$ & 120 & 44 \\
\hline 21 & $\mathrm{CuCl}_{2}$ & $\mathrm{LaCl}_{3}$ & THF & 120 & 32 \\
\hline 22 & $\mathrm{CuCl}_{2}$ & $\mathrm{CeCl}_{3}$ & THF & 120 & 40 \\
\hline 23 & $\mathrm{CuCl}_{2}$ & $\mathrm{InCl}_{3}$ & THF & 120 & trace \\
\hline $24^{b}$ & $\mathrm{CuCl}_{2}$ & $\mathrm{AlCl}_{3}$ & $\mathrm{CH}_{3} \mathrm{CN}$ & 120 & 31 \\
\hline 25 & $\mathrm{CuCl}_{2}$ & $\mathrm{AlCl}_{3}$ & $\mathrm{CH}_{3} \mathrm{CN}$ & 120 & 80 \\
\hline $26^{c}$ & $\mathrm{CuCl}_{2}$ & $\mathrm{AlCl}_{3}$ & $\mathrm{CH}_{3} \mathrm{CN}$ & 120 & 80 \\
\hline 27 & $\mathrm{CuCl}_{2}$ & $\mathrm{AlCl}_{3}$ & DMSO & 120 & 59 \\
\hline 28 & $\mathrm{CuCl}_{2}$ & $\mathrm{AlCl}_{3}$ & $\mathrm{PhMe}$ & 120 & 45 \\
\hline 29 & $\mathrm{CuCl}_{2}$ & $\mathrm{AlCl}_{3}$ & DMF & 120 & 53 \\
\hline 30 & $\mathrm{CuCl}_{2}$ & $\mathrm{AlCl}_{3}$ & dioxane & 120 & 41 \\
\hline 31 & $\mathrm{CuCl}_{2}$ & $\mathrm{AlCl}_{3}$ & DCE & 120 & 69 \\
\hline $32^{d}$ & $\mathrm{CuCl}_{2}$ & $\mathrm{AlCl}_{3}$ & $\mathrm{CH}_{3} \mathrm{CN}$ & 120 & 73 \\
\hline $33^{e}$ & $\mathrm{CuCl}_{2}$ & $\mathrm{AlCl}_{3}$ & $\mathrm{CH}_{3} \mathrm{CN}$ & 120 & 86 \\
\hline $34^{f}$ & $\mathrm{CuCl}_{2}$ & $\mathrm{AlCl}_{3}$ & $\mathrm{CH}_{3} \mathrm{CN}$ & 120 & 86 \\
\hline
\end{tabular}

${ }^{[\mathrm{a}]}$ Reactions conditions: $1(0.2 \mathrm{mmol}), 2(0.3 \mathrm{mmol}),[\mathrm{Cu}](0.4 \mathrm{mmol})$, additive $(0.2$ mmol) and solvent $(2.0 \mathrm{~mL})$, under air atmosphere, $10 \mathrm{~h}$, isolated yield. ${ }^{b} \mathrm{CuCl}_{2}(0.1$ mmol). ${ }^{c} \mathrm{AlCl}_{3}(0.06 \mathrm{mmol}) .{ }^{d} 24 \mathrm{~h} .{ }^{e}$ Under $\mathrm{N}_{2}$ atmosphere. ${ }^{f}$ Under $\mathrm{O}_{2}$ atmosphere. 
Table S2. Reaction Optimization for Ring-Opening Iodization ${ }^{[a]}$<smiles>O=C(OCCCCI)/C(=C(\I)c1ccccc1)c1ccccc1</smiles>

$\begin{array}{ccccc}\text { entry } & \text { I source } & \text { solvent } & \mathrm{T}\left({ }^{\circ} \mathrm{C}\right) & \text { yield }(\%) \\ 1 & \mathrm{CuI} & \mathrm{THF} & 120 & 0 \\ 2 & \mathrm{I}_{2} & \mathrm{THF} & 120 & 41 \\ 3 & \mathrm{NiI}_{2} & \mathrm{THF} & 120 & 20 \\ 4 & \mathrm{ZnI}_{2} & \mathrm{THF} & 120 & 11 \\ 5 & \mathrm{CdI}_{2} & \mathrm{THF} & 120 & 0 \\ 6 & \mathrm{AlI}_{3} & \mathrm{THF} & 120 & 40 \\ 7 & \mathrm{NIS} & \mathrm{THF} & 120 & 35 \\ 8 & \mathrm{CsI} & \mathrm{THF} & 120 & 0 \\ 9 & \mathrm{AgI} & \mathrm{THF} & 120 & \text { trace } \\ 10 & \mathrm{I} & \mathrm{THF} & 100 & 37 \\ 11 & \mathrm{I}_{2} & \mathrm{THF} & 80 & 32 \\ 12 & \mathrm{I}_{2} & \mathrm{THF} & 60 & 21 \\ 13^{b} & \mathrm{I}_{2} & \mathrm{CH}{ }_{3} \mathrm{CN} & 120 & 23 \\ 14^{c} & \mathrm{I}_{2} & \mathrm{CH}{ }_{3} \mathrm{CN} & 120 & 44 \\ 15 & \mathrm{I}_{2} & \mathrm{CH}{ }_{3} \mathrm{CN} & 120 & 45 \\ 16 & \mathrm{I}_{2} & \mathrm{DMSO} & 120 & 22 \\ 17 & \mathrm{I}_{2} & \mathrm{PhMe} & 120 & 31 \\ 18 & \mathrm{I}_{2} & \mathrm{DMF} & 120 & 26 \\ 19 & \mathrm{I}_{2} & \text { dioxane } & 120 & 19 \\ 20 & \mathrm{I}_{2} & \mathrm{DCE} & 120 & 38 \\ 21^{d} & \mathrm{I}_{2} & \mathrm{CH}{ }_{3} \mathrm{CN} & 120 & 39 \\ 22^{e} & \mathrm{I}_{2} & \mathrm{CH}{ }_{3} \mathrm{CN} & 120 & 45 \\ 23^{f} & \mathrm{I}_{2} & \mathrm{CH}{ }_{3} \mathrm{CN} & 120 & 45\end{array}$

${ }^{[\mathrm{a}]}$ Reactions conditions: $1(0.2 \mathrm{mmol}), 2(0.3 \mathrm{mmol})$, I source $(0.4 \mathrm{mmol})$, and solvent $(2.0 \mathrm{~mL})$, under air atmosphere, $10 \mathrm{~h}$, isolated yield. ${ }^{b} \mathrm{I}_{2}(0.2 \mathrm{mmol}) .{ }^{c} \mathrm{I}_{2}(1 \mathrm{mmol}) .{ }^{d} 24$ h. ${ }^{e}$ Under $\mathrm{N}_{2}$ atmosphere. ${ }^{f}$ Under $\mathrm{O}_{2}$ atmosphere. 


\section{General Experimental Procedures}

(1) General procedure for bromination $(\operatorname{method} A, 1 a-23 a)$

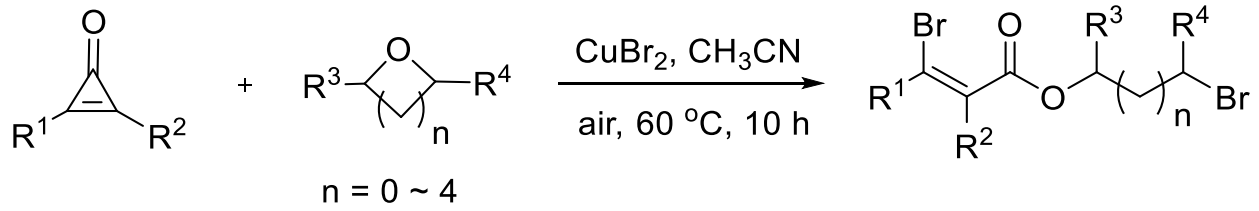

\section{Scheme S1}

A $10 \mathrm{~mL}$ pressure tube equipped with a stir bar was charged with cyclopropenones ( $0.2 \mathrm{mmol}, 1.0$ equiv.), $\mathrm{CuBr}_{2}(0.4 \mathrm{mmol}, 2.0$ equiv), saturated oxygen heterocycle ( 0.3 mmol, 1.5 equiv.) and $\mathrm{CH}_{3} \mathrm{CN}(2 \mathrm{~mL})$. The reaction mixture was stirred in a heating mantle preheated to $60{ }^{\circ} \mathrm{C}$ under air atmosphere for $10 \mathrm{~h}$. After completion of the reaction, the reaction mixture was concentrated under reduced pressure, then $2.0 \mathrm{ml}$ of water was added, and the reaction solution was extracted with ethyl acetate $(3 \times 2 \mathrm{~mL})$, the combined organic layers were dried over anhydrous magnesium sulfate, filtered and evaporated under reduced pressure. The remaining residue was purified by column chromatography on silica gel to afford the corresponding products $(\mathbf{1 a}-\mathbf{2 3 a})$.

(2) General procedure for chlorination $(\operatorname{method} B, 1 \mathrm{~b}-17 \mathrm{~b})$

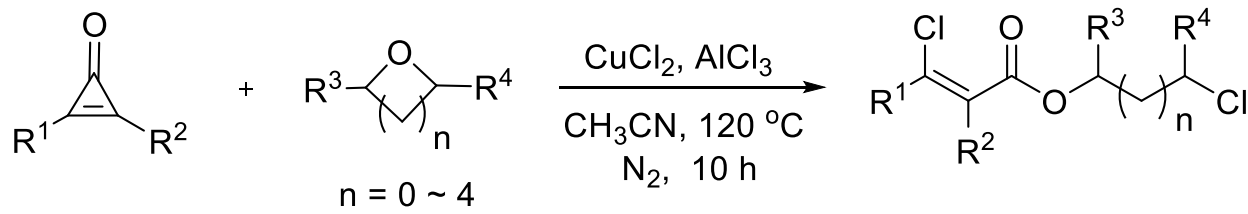

\section{Scheme S2}

Under a nitrogen atmosphere, a $10 \mathrm{~mL}$ pressure tube equipped with a stir bar was charged with cyclopropenones ( $0.2 \mathrm{mmol}, 1.0$ equiv. $), \mathrm{CuCl}_{2}(0.4 \mathrm{mmol}, 2.0$ equiv.), saturated oxygen heterocycle ( $0.3 \mathrm{mmol}, 1.5$ equiv.), $\mathrm{AlCl}_{3}(30 \mathrm{~mol} \%)$ and $\mathrm{CH}_{3} \mathrm{CN}(2$ $\mathrm{mL}$ ). The reaction mixture was stirred in a heating mantle preheated to $120{ }^{\circ} \mathrm{C}$ for $10 \mathrm{~h}$. After completion of the reaction, the reaction mixture was concentrated under reduced pressure, then $2.0 \mathrm{ml}$ of water was added, and the reaction solution was extracted with ethyl acetate $(3 \times 2 \mathrm{~mL})$, the combined organic layers were dried over anhydrous magnesium sulfate, filtered and evaporated under reduced pressure. The remaining residue was purified by column chromatography on silica gel to afford the corresponding products $(\mathbf{1 b}-\mathbf{1 7 b})$.

\section{(3) General procedure for iodization $(\operatorname{method} \mathrm{C}, 1 \mathrm{c}-6 \mathrm{c})$}

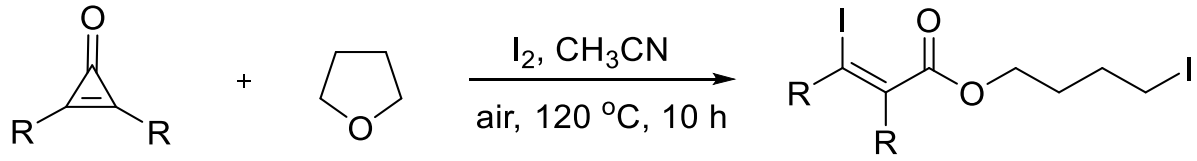

\section{Scheme S3}


A $10 \mathrm{~mL}$ pressure tube equipped with a stir bar was charged with cyclopropenones (0.2 mmol, 1.0 equiv.), $\mathrm{I}_{2}$ ( $0.4 \mathrm{mmol}, 2.0$ equiv.), saturated oxygen heterocycle ( 0.3 mmol, 1.5 equiv.) and $\mathrm{CH}_{3} \mathrm{CN}(2 \mathrm{~mL})$. The reaction mixture was stirred in a heating mantle preheated to $120{ }^{\circ} \mathrm{C}$ under air atmosphere for $10 \mathrm{~h}$. After completion of the reaction, the reaction mixture was concentrated under reduced pressure, then $2.0 \mathrm{ml}$ of water was added, and the reaction solution was extracted with ethyl acetate $(3 \times 2 \mathrm{~mL})$, the combined organic layers were dried over anhydrous magnesium sulfate, filtered and evaporated under reduced pressure. The remaining residue was purified by column chromatography on silica gel to afford the corresponding products $(\mathbf{1 c}-\mathbf{6 c})$.

\section{(4) Procedure for $5.0 \mathrm{mmol}$ scale reaction}

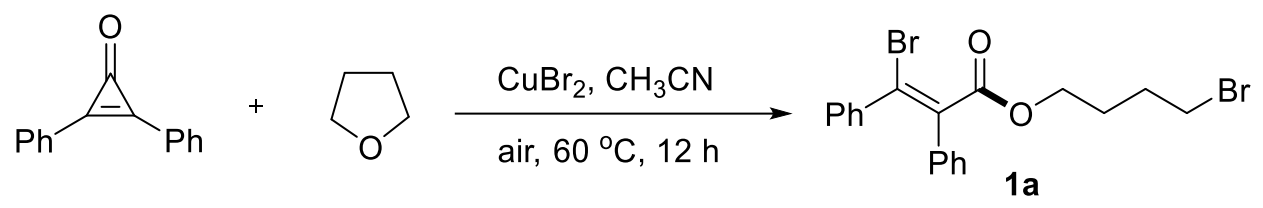

\section{Scheme S4}

A $100 \mathrm{~mL}$ pressure tube equipped with a stir bar was charged with diphenylcyclopropenone ( $5 \mathrm{mmol}, 1.0$ equiv.), $\mathrm{CuBr}_{2}$ (10 mmol, 2.0 equiv.), saturated oxygen heterocycle (7.5 mmol, 1.5 equiv.) and $\mathrm{CH}_{3} \mathrm{CN}(30 \mathrm{~mL})$. The reaction mixture was stirred in a heating mantle preheated to $60{ }^{\circ} \mathrm{C}$ under air atmosphere for $10 \mathrm{~h}$. After completion of the reaction, the reaction mixture was concentrated under reduced pressure, then $30 \mathrm{ml}$ of water was added, and the reaction solution was extracted with ethyl acetate $(3 \times 30 \mathrm{~mL})$, the combined organic layers were dried over anhydrous magnesium sulfate, filtered and evaporated under reduced pressure. The remaining residue was purified by column chromatography on silica gel to afford $1 \mathbf{a}(1.62 \mathrm{~g}, 74 \%)$.

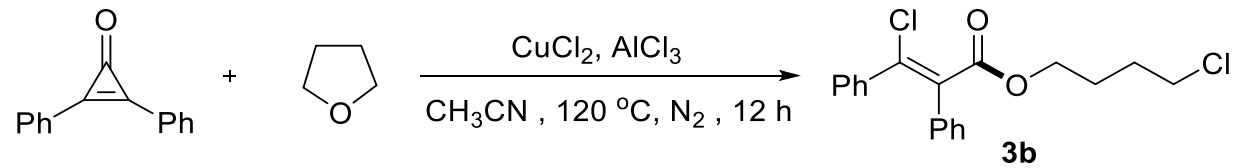

\section{Scheme S5}

Under a nitrogen atmosphere, a $100 \mathrm{~mL}$ pressure tube equipped with a stir bar was charged with diphenylcyclopropenone (5 mmol, 1.0 equiv.), $\mathrm{CuCl}_{2}$ (10 mmol, 2.0 equiv.), saturated oxygen heterocycle ( $7.5 \mathrm{mmol}, 1.5$ equiv.), $\mathrm{AlCl}_{3}(30 \mathrm{~mol} \%)$ and $\mathrm{CH}_{3} \mathrm{CN}(30 \mathrm{~mL})$. The reaction mixture was stirred in a heating mantle preheated to 120 ${ }^{\circ} \mathrm{C}$ for $10 \mathrm{~h}$. After completion of the reaction, the reaction mixture was concentrated under reduced pressure, then $30 \mathrm{ml}$ of water was added, and the reaction solution was extracted with ethyl acetate $(3 \times 30 \mathrm{~mL})$, the combined organic layers were dried over anhydrous magnesium sulfate, filtered and evaporated under reduced pressure. The remaining residue was purified by column chromatography on silica gel to afford $\mathbf{3 b}$ $(1.41 \mathrm{~g}, 81 \%)$ 


\section{Characterization of Products in Details}

\section{4-bromobutyl (Z)-3-bromo-2,3-diphenylacrylate (1a)}<smiles>O=C(OCCCCBr)/C(=C(\Br)c1ccccc1)c1ccccc1</smiles>

The general procedure (Method A) was followed, and 20/1 hexane/ethyl acetate was used as the eluant to afford colorless oil (71.9 mg, 82\%), IR: 2959, 1723, 1172, 695, 556 $\mathrm{cm}^{-1} .{ }^{1} \mathrm{H}$ NMR $(500 \mathrm{MHz}$, Chloroform- $d$ ) $\delta 7.25-7.23(\mathrm{~m}, 2 \mathrm{H}), 7.22-7.14(\mathrm{~m}, 6 \mathrm{H})$, $7.12-7.09(\mathrm{~m}, 2 \mathrm{H}), 4.31(\mathrm{t}, J=6.2 \mathrm{~Hz}, 2 \mathrm{H}), 3.41(\mathrm{t}, J=6.5 \mathrm{~Hz}, 2 \mathrm{H}), 2.00-1.94(\mathrm{~m}$, 2H), $1.92-1.85(\mathrm{~m}, 2 \mathrm{H}) .{ }^{13} \mathrm{C}$ NMR $(125 \mathrm{MHz}$, Chloroform- $d$ ) $\delta$ 167.8, 138.2, 137.1, 134.9, 129.7, 128.8, 128.8, 128.4, 128.1, 128.1, 123.7, 64.7, 32.9, 29.2, 27.2. HRMS: (ESI) calculated for $\mathrm{C}_{19} \mathrm{H}_{19} \mathrm{Br}_{2} \mathrm{O}_{2}[\mathrm{M}+\mathrm{H}]^{+} 436.9746$, found 436.9749 .

\section{4-bromobutyl (Z)-3-bromo-2,3-bis(4-fluorophenyl)acrylate (2a)}<smiles>O=C(OCCCCBr)/C(=C(\Br)c1ccc(F)cc1)c1ccc(F)cc1</smiles>

The general procedure (Method A) was followed, and 20/1 hexane/ethyl acetate was used as the eluant to afford colorless oil (86.3 mg, 91\%), IR: 2961, 1725, 1503, 1157, $539 \mathrm{~cm}^{-1} .{ }^{1} \mathrm{H}$ NMR $(500 \mathrm{MHz}$, Chloroform- $d$ ) $\delta 7.22(\mathrm{dd}, J=8.6,5.4 \mathrm{~Hz}, 2 \mathrm{H}), 7.08(\mathrm{dd}$, $J=8.6,5.4 \mathrm{~Hz}, 2 \mathrm{H}), 6.92-6.87(\mathrm{~m}, 4 \mathrm{H}), 4.31(\mathrm{t}, J=6.2 \mathrm{~Hz}, 2 \mathrm{H}), 3.42(\mathrm{t}, J=6.6 \mathrm{~Hz}$, $3 \mathrm{H}), 2.00-1.94(\mathrm{~m}, 2 \mathrm{H}), 1.93-1.87(\mathrm{~m}, 2 \mathrm{H}) .{ }^{13} \mathrm{C}$ NMR $(125 \mathrm{MHz}$, Chloroform- $d$ ) $\delta$ $167.5,162.6(J=248.8 \mathrm{~Hz}), 162.3(J=248.8 \mathrm{~Hz}), 136.3,134.1(J=3.8 \mathrm{~Hz}), 131.2(J$ $=128.8 \mathrm{~Hz}), 131.1(J=128.8 \mathrm{~Hz}), 130.7(J=3.8 \mathrm{~Hz}), 122.8,115.6(J=36.2 \mathrm{~Hz}), 115.4$ $(J=36.2 \mathrm{~Hz}), 64.8,32.8,29.2,27.1 .{ }^{19} \mathrm{~F}$ NMR (471 MHz, Chloroform- $d$ ) $\delta-110.8$, 112.1. HRMS: (ESI) calculated for $\mathrm{C}_{19} \mathrm{H}_{17} \mathrm{Br}_{2} \mathrm{~F}_{2} \mathrm{O}_{2}[\mathrm{M}+\mathrm{H}]^{+} 472.9558$, found 472.9553 .

\section{4-bromobutyl (Z)-3-bromo-2,3-bis(4-bromophenyl)acrylate (3a)}<smiles>O=C(OCCCCBr)/C(Br)=C(\Br)c1ccc(Br)cc1</smiles>

The general procedure (Method A) was followed, and 20/1 hexane/ethyl acetate was used as the eluant to afford colorless oil (94.2 mg, 79\%), IR: 2959, 1724, 1010, 735, $496 \mathrm{~cm}^{-1} .{ }^{1} \mathrm{H}$ NMR (500 MHz, Chloroform- $d$ ) $\delta 7.35$ (dd, $\left.J=10.6,8.2 \mathrm{~Hz}, 4 \mathrm{H}\right), 7.10$ 
$(\mathrm{d}, J=8.3 \mathrm{fHz}, 2 \mathrm{H}), 6.97(\mathrm{~d}, J=8.3 \mathrm{~Hz}, 2 \mathrm{H}), 4.31(\mathrm{t}, J=6.1 \mathrm{~Hz}, 2 \mathrm{H}), 3.42(\mathrm{t}, J=6.5$ $\mathrm{Hz}, 2 \mathrm{H}), 1.98-1.86(\mathrm{~m}, 4 \mathrm{H}) .{ }^{13} \mathrm{C}$ NMR $(125 \mathrm{MHz}$, Chloroform- $d$ ) $\delta 167.2,136.8,136.5$, 133.5, 131.9, 131.6, 131.2, 130.3, 123.5, 123.0, 122.8, 65.0, 32.8, 29.2, 27.1. HRMS: (ESI) calculated for $\mathrm{C}_{19} \mathrm{H}_{17} \mathrm{Br}_{4} \mathrm{O}_{2}[\mathrm{M}+\mathrm{H}]^{+}$592.7957, found 592.7954 .

\section{4-bromobutyl (Z)-3-bromo-2,3-di-p-tolylacrylate (4a)}<smiles>Cc1ccc(C(Br)=C(C(=O)OCCCCBr)c2ccc(C)cc2)cc1</smiles>

The general procedure (Method A) was followed, and 20/1 hexane/ethyl acetate was used as the eluant to afford colorless oil (69.9 mg, 75\%), IR: 2921, 1725, 1169, 818, $544 \mathrm{~cm}^{-1} .{ }^{1} \mathrm{H}$ NMR $(500 \mathrm{MHz}$, Chloroform- $d) \delta 7.15(\mathrm{~d}, J=7.9 \mathrm{~Hz}, 2 \mathrm{H}), 7.02-6.96(\mathrm{~m}$, $6 \mathrm{H}), 4.30(\mathrm{t}, J=6.2 \mathrm{~Hz}, 2 \mathrm{H}), 3.41(\mathrm{t}, J=5.6 \mathrm{~Hz}, 2 \mathrm{H}), 2.28(\mathrm{~s}, 3 \mathrm{H}), 2.25(\mathrm{~s}, 3 \mathrm{H}), 1.99-$ $1.94(\mathrm{~m}, 2 \mathrm{H}), 1.91-1.87$ (m, 2H). ${ }^{13} \mathrm{C}$ NMR (125 MHz, Chloroform- $d$ ) $\delta$ 168.1, 138.9, 138.0, 136.5, 135.4, 132.1, 129.7, 129.1, 128.8, 128.6, 123.2, 64.6, 32.9, 29.2, 27.2, 21.2, 21.2. HRMS: (ESI) calculated for $\mathrm{C}_{21} \mathrm{H}_{23} \mathrm{Br}_{2} \mathrm{O}_{2}[\mathrm{M}+\mathrm{H}]^{+} 465.0059$, found 465.0053 .

\section{4-bromobutyl (Z)-3-bromo-2,3-bis(4-(tert-butyl)phenyl)acrylate (5a)}<smiles>CC(C)(C)c1ccc(C(Br)=C(C(=O)OCCCCBr)c2ccc(C(C)(C)C)cc2)cc1</smiles>

The general procedure (Method A) was followed, and 20/1 hexane/ethyl acetate was used as the eluant to afford colorless oil (84.8 mg, 77\%), IR: 2961, 1726, 1175, 838, $558 \mathrm{~cm}^{-1} .{ }^{1} \mathrm{H}$ NMR $(500 \mathrm{MHz}$, Chloroform- $d) \delta 7.22-7.15(\mathrm{~m}, 6 \mathrm{H}), 7.02(\mathrm{~d}, J=8.5 \mathrm{~Hz}$, $2 \mathrm{H}), 4.31(\mathrm{t}, J=6.2 \mathrm{~Hz}, 2 \mathrm{H}), 3.41(\mathrm{t}, J=6.4 \mathrm{~Hz}, 2 \mathrm{H}), 2.01-1.97(\mathrm{~m}, 2 \mathrm{H}), 1.92-1.85$ $(\mathrm{m}, 2 \mathrm{H}), 1.26(\mathrm{~s}, 9 \mathrm{H}), 1.24$ (s, 9H). ${ }^{13} \mathrm{C}$ NMR $(125 \mathrm{MHz}$, Chloroform- $d$ ) $\delta$ 168.2, 152.0, 151.1, 135.3, 131.9, 129.5, 128.4, 125.2, 124.9, 123.3, 64.6, 34.6, 34.5, 32.9, 31.1, 29.3, 27.2. HRMS: (ESI) calculated for $\mathrm{C}_{27} \mathrm{H}_{35} \mathrm{Br}_{2} \mathrm{O}_{2}[\mathrm{M}+\mathrm{H}]^{+}$549.0998, found 549.0997.

\section{4-bromobutyl (Z)-3-bromo-2,3-bis(3-bromophenyl)acrylate (6a)}<smiles>O=C(OCCCCBr)/C(=C(\Br)c1cccc(Br)c1)c1cccc(Br)c1</smiles>

The general procedure (Method A) was followed, and 20/1 hexane/ethyl acetate was used as the eluant to afford colorless oil (97.8 mg, 82\%), IR:2955, 1725, 1098, 816, 470 
$\mathrm{cm}^{-1} .{ }^{1} \mathrm{H}$ NMR (400 MHz, Chloroform- $d$ ) $\delta 7.49-7.48(\mathrm{~m}, 1 \mathrm{H}), 7.41-7.38(\mathrm{~m}, 2 \mathrm{H})$, $7.35-7.34(\mathrm{~m}, 1 \mathrm{H}), 7.16-7.06(\mathrm{~m}, 3 \mathrm{H}), 7.04-7.02(\mathrm{~m}, 1 \mathrm{H}), 4.35(\mathrm{t}, J=6.1 \mathrm{~Hz}, 2 \mathrm{H})$, $3.46(\mathrm{t}, J=6.4 \mathrm{~Hz}, 2 \mathrm{H}), 2.03-1.97(\mathrm{~m}, 2 \mathrm{H}), 1.97-1.89(\mathrm{~m}, 2 \mathrm{H}) .{ }^{13} \mathrm{C}$ NMR $(100 \mathrm{MHz}$, Chloroform- $d$ ) $\delta 167.0,139.7,136.6,136.3,132.6,132.3,131.6,131.5,130.1,129.7$, 128.2, 127.7, 123.1, 122.5, 122.2, 65.1, 32.9, 29.2, 27.2. HRMS: (ESI) calculated for $\mathrm{C}_{19} \mathrm{H}_{17} \mathrm{Br}_{4} \mathrm{O}_{2}[\mathrm{M}+\mathrm{H}]^{+}$592.7957, found 592.7957 .

\section{4-bromobutyl (Z)-3-bromo-2,3-bis(3-chlorophenyl)acrylate (7a)}<smiles>O=C(OCCCCBr)/C(Br)=C(\Br)c1cccc(Cl)c1</smiles>

The general procedure (Method A) was followed, and 20/1 hexane/ethyl acetate was used as the eluant to afford colorless oil (87.3 mg, 86\%), IR: 2959, 1723, 1176, 812, $409 \mathrm{~cm}^{-1} .{ }^{1} \mathrm{H}$ NMR (400 MHz, Chloroform- $d$ ) $\delta 7.31-7.26(\mathrm{~m}, 1 \mathrm{H}), 7.25-7.17(\mathrm{~m}$, $2 \mathrm{H}), 7.15-7.10(\mathrm{~m}, 3 \mathrm{H}), 7.07-7.02(\mathrm{~m}, 1 \mathrm{H}), 6.99-6.91(\mathrm{~m}, 1 \mathrm{H}), 4.32(\mathrm{t}, J=6.0 \mathrm{~Hz}$, 2H), $3.42(\mathrm{t}, J=6.2 \mathrm{~Hz}, 2 \mathrm{H}), 2.01-1.94(\mathrm{~m}, 2 \mathrm{H}), 1.93-1.87(\mathrm{~m}, 2 \mathrm{H}) .{ }^{13} \mathrm{C}$ NMR $(100$ MHz, Chloroform- $d$ ) $\delta$ 167.0, 139.6, 136.7, 136.1, 134.5, 134.3, 129.8, 129.7, 129.5, 129.4, 128.7, 128.7, 127.8, 127.2, 123.2, 65.1, 32.9, 29.2, 27.2. HRMS: (ESI) calculated for $\mathrm{C}_{19} \mathrm{H}_{17} \mathrm{Br}_{2} \mathrm{Cl}_{2} \mathrm{O}_{2}[\mathrm{M}+\mathrm{H}]^{+}$504.8967, found 504.8966.

\section{4-bromobutyl (Z)-3-bromo-2-ethylpent-2-enoate (8a)}<smiles>CCC(CC)=C(Br)C(=O)OCCCCBr</smiles>

The general procedure (Method A) was followed, and 20/1 hexane/ethyl acetate was used as the eluant to afford colorless oil (49.3 mg, 72\%), IR: 2971, 1724, 1150, 892, $651 \mathrm{~cm}^{-1} .{ }^{1} \mathrm{H}$ NMR $(500 \mathrm{MHz}$, Chloroform- $d$ ) $\delta 4.24(\mathrm{t}, J=5.9 \mathrm{~Hz}, 2 \mathrm{H}), 3.58(\mathrm{t}, J=6.1$ $\mathrm{Hz}, 2 \mathrm{H}), 2.46-2.42(\mathrm{~m}, 2 \mathrm{H}), 2.37-2.32(\mathrm{~m}, 2 \mathrm{H}), 1.94-1.84(\mathrm{~m}, 4 \mathrm{H}), 1.17(\mathrm{t}, J=7.5$ $\mathrm{Hz}, 3 \mathrm{H}), 1.06$ (t, $J=7.6 \mathrm{~Hz}, 3 \mathrm{H}) .{ }^{13} \mathrm{C}$ NMR $(125 \mathrm{MHz}$, Chloroform- $d$ ) $\delta 168.0,136.8$, 131.7, 64.0, 44.3, 29.1, 28.6, 26.0, 24.0, 13.2, 12.2. HRMS: (ESI) calculated for $\mathrm{C}_{11} \mathrm{H}_{19} \mathrm{Br}_{2} \mathrm{O}_{2}[\mathrm{M}+\mathrm{H}]^{+}$340.9746, found 340.9747.

4-bromobutyl (Z)-3-bromo-2-(naphthalen-2-yl)acrylate and 4-bromobutyl ( $Z$ )-3bromo-3-(naphthalen-2-yl)acrylate (9a and 9a')<smiles>O=C(/C=C(\Br)c1ccc2ccccc2c1)OCCCCBr</smiles> 
The general procedure (Method A) was followed, and 20/1 hexane/ethyl acetate was used as the eluant to afford colorless oil (60.2 mg, 73\%), IR: 2959, 1715, 1223, 743, $474 \mathrm{~cm}^{-1} .{ }^{1} \mathrm{H}$ NMR $(500 \mathrm{MHz}$, Chloroform- $d) \delta 7.87-7.80(\mathrm{~m}, 3 \mathrm{H}), 7.78-7.77(\mathrm{~m}$, $1 \mathrm{H}), 7.52-7.48(\mathrm{~m}, 2 \mathrm{H}), 7.45-7.43(\mathrm{~m}, 1 \mathrm{H}), 6.92(\mathrm{~s}, 1 \mathrm{H}), 4.39(\mathrm{t}, J=6.2 \mathrm{~Hz}, 1 \mathrm{H})$, $4.23(\mathrm{t}, J=6.2 \mathrm{~Hz}, 1 \mathrm{H}), 3.42(\mathrm{t}, J=6.5 \mathrm{~Hz}, 1 \mathrm{H}), 3.36(\mathrm{t}, J=6.5 \mathrm{~Hz}, 1 \mathrm{H}), 2.04-1.93$ $(\mathrm{m}, 2 \mathrm{H}), 1.93-1.85(\mathrm{~m}, 2 \mathrm{H}) .{ }^{13} \mathrm{C}$ NMR $(125 \mathrm{MHz}$, Chloroform- $d) \delta 166.6,140.9,133.2$, 133.1, 132.3, 128.9, 128.6, 128.3, 128.2, 127.7, 127.7, 126.8, 126.7, 126.5, 126.2, 126.1, 124.4, 123.7, 109.3, 64.7, 32.8, 32.8, 29.2, 27.2, 27.2. HRMS: (ESI) calculated for $\mathrm{C}_{17} \mathrm{H}_{17} \mathrm{Br}_{2} \mathrm{O}_{2}[\mathrm{M}+\mathrm{H}]^{+}$410.9590, found 410.9588 .

4-bromobutyl (Z)-3-bromo-2-methyl-3-phenylacrylate and 4-bromobutyl (E)-3bromo-2-phenylbut-2-enoate (10a and 10a')<smiles>C/C(C(=O)OCCCCBr)=C(\Br)c1ccccc1</smiles><smiles>C/C(Br)=C(\C(=O)OCCCCBr)c1ccccc1</smiles>

The general procedure (Method A) was followed, and 20/1 hexane/ethyl acetate was used as the eluant to afford colorless oil (58.0 mg, 77\%), IR: 2959, 1724, 1204, 708, $560 \mathrm{~cm}^{-1} .{ }^{1} \mathrm{H}$ NMR $(500 \mathrm{MHz}$, Chloroform- $d$ ) $\delta 7.41-7.30(\mathrm{~m}, 3 \mathrm{H}), 7.31-7.25(\mathrm{~m}$, $2 \mathrm{H}), 4.21(\mathrm{t}, J=6.2 \mathrm{~Hz}, 2 \mathrm{H}), 3.37(\mathrm{t}, J=6.5 \mathrm{~Hz}, 2 \mathrm{H}), 2.31(\mathrm{~s}, 2 \mathrm{H}), 1.99-1.87(\mathrm{~m}, 3 \mathrm{H})$, $1.89-1.76(\mathrm{~m}, 2 \mathrm{H}) .{ }^{13} \mathrm{C}$ NMR $(125 \mathrm{MHz}$, Chloroform- $d$ ) $\delta 167.4,136.1,134.9,128.6$, 128.6, 128.4, 128.4, 128.3, 124.3, 64.4, 64.3, 32.9, 32.9, 29.3, 29.2, 27.2, 27.1, 26.1, 18.7. HRMS: (ESI) calculated for $\mathrm{C}_{14} \mathrm{H}_{17} \mathrm{Br}_{2} \mathrm{O}_{2}[\mathrm{M}+\mathrm{H}]^{+} 374.9590$, found 374.9586 .

\section{4-bromobutyl (Z)-2-(acetoxymethyl)-3-bromo-3-phenylacrylate (11a)}<smiles>CC(=O)OCC(C(=O)OCCCCBr)=C(Br)c1ccccc1</smiles>

The general procedure (Method A) was followed, and 20/1 hexane/ethyl acetate was used as the eluant to afford colorless oil (60.8 mg, 70\%), IR: 2960, 1727, 1197, 1025, $554 \mathrm{~cm}^{-1} .{ }^{1} \mathrm{H}$ NMR (400 MHz, Chloroform- $d$ ) $\delta 7.44-7.35(\mathrm{~m}, 3 \mathrm{H}), 7.32-7.29(\mathrm{~m}$, $2 \mathrm{H}), 4.75(\mathrm{~s}, 2 \mathrm{H}), 4.25(\mathrm{t}, J=6.0 \mathrm{~Hz}, 2 \mathrm{H}), 3.38(\mathrm{t}, J=6.4 \mathrm{~Hz}, 2 \mathrm{H}), 2.10(\mathrm{~s}, 3 \mathrm{H}), 1.94-$ $1.89(\mathrm{~m}, 2 \mathrm{H}), 1.89-1.81(\mathrm{~m}, 2 \mathrm{H}) .{ }^{13} \mathrm{C}$ NMR $(125 \mathrm{MHz}$, Chloroform- $d$ ) $\delta 170.1,166.7$, 140.7, 133.6, 129.2, 128.8, 128.2, 121.4, 64.9, 64.8, 32.8, 29.1, 27.1, 20.7. HRMS: (ESI) calculated for $\mathrm{C}_{16} \mathrm{H}_{19} \mathrm{Br}_{2} \mathrm{O}_{4}[\mathrm{M}+\mathrm{H}]^{+} 432.9645$, found 432.9642 .

5-bromopentan-2-yl (Z)-3-bromo-2,3-diphenylacrylate and 4-bromopentyl ( $Z$ )-3bromo-2,3-diphenylacrylate (12a and 12a')<smiles>CC(CCCBr)OC(=O)/C(=C(\Br)c1ccccc1)c1ccccc1</smiles><smiles>CC(Br)CCCOC(=O)/C(=C(\Br)c1ccccc1)c1ccccc1</smiles> 
The general procedure (Method A) was followed, and 20/1 hexane/ethyl acetate was used as the eluant to afford colorless oil (70.6 mg, 78\%), IR: 2963, 1722, 1172, 692, $557 \mathrm{~cm}^{-1} .{ }^{1} \mathrm{H}$ NMR $(500 \mathrm{MHz}$, Chloroform- $d) \delta 7.25-7.24(\mathrm{~m}, 2 \mathrm{H}), 7.19-7.14(\mathrm{~m}$, $6 \mathrm{H}), 7.11-7.09(\mathrm{~m}, 2 \mathrm{H}), 4.35-4.28(\mathrm{~m}, 1 \mathrm{H}), 4.14-4.08(\mathrm{~m}, 1 \mathrm{H}), 3.40-3.32(\mathrm{~m}, 1 \mathrm{H})$, $1.99-1.76(\mathrm{~m}, 4 \mathrm{H}), 1.68(\mathrm{~d}, J=6.6 \mathrm{~Hz}, 2 \mathrm{H}), 1.34(\mathrm{~d}, J=6.2 \mathrm{~Hz}, 1 \mathrm{H}) .{ }^{13} \mathrm{C}$ NMR $(125$ MHz, Chloroform- $d$ ) $\delta 167.8,138.3,135.0,129.8,129.7,128.8,128.8,128.7,128.4$, 128.1, 128.1, 123.7, 71.8, 64.8, 50.5, 37.4, 34.3, 33.1, 28.5, 26.9, 26.4, 19.8. HRMS: (ESI) calculated for $\mathrm{C}_{20} \mathrm{H}_{21} \mathrm{Br}_{2} \mathrm{O}_{2}[\mathrm{M}+\mathrm{H}]^{+} 450.9903$, found 450.9909 .

\section{1,5-dibromopentan-2-yl (Z)-3-bromo-2,3-diphenylacrylate (13a)}<smiles>O=C(OC(CBr)CCCBr)C(=C(Br)c1ccccc1)c1ccccc1</smiles>

The general procedure (Method A) was followed, and 20/1 hexane/ethyl acetate was used as the eluant to afford colorless oil (59.5mg, 56\%), IR: 2937, 1722, 1179, 692, 551 $\mathrm{cm}^{-1} .{ }^{1} \mathrm{H}$ NMR (500 MHz, Chloroform- $d$ ) $\delta 7.25-7.24(\mathrm{~m}, 2 \mathrm{H}), 7.20-7.17(\mathrm{~m}, 6 \mathrm{H})$, $7.13-7.11(\mathrm{~m}, 2 \mathrm{H}), 5.24-5.20(\mathrm{~m}, 1 \mathrm{H}), 3.54(\mathrm{~d}, J=5.2 \mathrm{~Hz}, 2 \mathrm{H}), 3.38(\mathrm{t}, J=6.0 \mathrm{~Hz}$, 2H), $2.02-1.91(\mathrm{~m}, 4 \mathrm{H}) .{ }^{13} \mathrm{C}$ NMR (125 MHz, Chloroform- $d$ ) $\delta 167.1,138.0,136.7$, 134.7, 129.7, 128.9, 128.9, 128.4, 128.2, 128.1, 124.0, 73.2, 32.8, 32.6, 31.0, 28.1. HRMS: (ESI) calculated for $\mathrm{C}_{20} \mathrm{H}_{20} \mathrm{Br}_{3} \mathrm{O}_{2}[\mathrm{M}+\mathrm{H}]^{+} 528.9008$, found 528.9008 .

\section{2-bromocyclopentyl (Z)-3-bromo-2,3-diphenylacrylate (14a)}<smiles>O=C(O[C@H]1CCC[C@H]1Br)/C(=C(\Br)c1ccccc1)c1ccccc1</smiles>

The general procedure (Method A) was followed, and 20/1 hexane/ethyl acetate was used as the eluant to afford colorless oil (48.7 mg, 54\%), IR: 2924, 1725, 1171, 692, $555 \mathrm{~cm}^{-1} .{ }^{1} \mathrm{H}$ NMR $(500 \mathrm{MHz}$, Chloroform- $d$ ) $\delta$ 7.25- $7.23(\mathrm{~m}, 3 \mathrm{H}), 7.21-7.16(\mathrm{~m}, 5 \mathrm{H})$, $7.10-7.08(\mathrm{~m}, 2 \mathrm{H}), 5.46(\mathrm{~d}, J=5.9 \mathrm{~Hz}, 1 \mathrm{H}), 4.41-4.35(\mathrm{~m}, 1 \mathrm{H}), 2.42-2.32(\mathrm{~m}, 2 \mathrm{H})$, $2.12-2.07(\mathrm{~m}, 1 \mathrm{H}), 2.01-1.95(\mathrm{~m}, 1 \mathrm{H}), 1.91-1.85(\mathrm{~m}, 2 \mathrm{H}) .{ }^{13} \mathrm{C}$ NMR $(125 \mathrm{MHz}$, Chloroform- $d$ ) $\delta 166.8,138.2,136.7,134.7,129.7,128.9,128.8,128.4,128.2,128.1$, 123.8, 83.5, 52.5, 34.6, 29.0, 21.6. HRMS: (ESI) calculated for $\mathrm{C}_{20} \mathrm{H}_{19} \mathrm{Br}_{2} \mathrm{O}_{2}[\mathrm{M}+\mathrm{H}]^{+}$ 448.9746 , found 448.9740 .

\section{6-bromo-2,2-dimethyl-1,3-dioxepan-5-yl (Z)-3-bromo-2,3-diphenylacrylate (15a)}<smiles>CC1(C)OCC(Br)[C@H](OC(=O)/C(Br)=C(\Br)c2ccccc2)CO1</smiles>

The general procedure (Method A) was followed, and 20/1 hexane/ethyl acetate was used as the eluant to afford colorless oil (43.0 mg, 42\%), IR: 3363, 1726, 1169, 691, $555 \mathrm{~cm}^{-1} .{ }^{1} \mathrm{H}$ NMR $(500 \mathrm{MHz}$, Chloroform- $d) \delta 7.25-7.23(\mathrm{~m}, 2 \mathrm{H}), 7.21-7.15(\mathrm{~m}$, 
$6 \mathrm{H}), 7.13-7.11(\mathrm{~m}, 2 \mathrm{H}), 4.64-4.55(\mathrm{~m}, \mathrm{,} 2 \mathrm{H}), 4.33-4.30(\mathrm{~m}, 1 \mathrm{H}), 4.24-4.20(\mathrm{~m}$, $1 \mathrm{H}), 4.05-4.02(\mathrm{~m}, 1 \mathrm{H}), 3.87(\mathrm{dd}, J=8.6,6.2 \mathrm{~Hz}, 1 \mathrm{H}), 1.47(\mathrm{~s}, 3 \mathrm{H}), 1.34(\mathrm{~s}, 3 \mathrm{H}) .{ }^{13} \mathrm{C}$ NMR (125 MHz, Chloroform-d) $\delta$ 167.0, 138.1, 136.4, 134.7, 129.7, 128.9, 128.9, 128.4, 128.2, 128.1, 124.6, 110.2, 75.1, 67.1, 65.9, 49.6, 26.1, 25.2. HRMS: (ESI) calculated for $\mathrm{C}_{22} \mathrm{H}_{23} \mathrm{Br}_{2} \mathrm{O}_{4}[\mathrm{M}+\mathrm{H}]^{+}$508.9958, found 508.9956 .

1-bromopropan-2-yl (Z)-3-bromo-2,3-diphenylacrylate and 2-bromopropyl (Z)-3bromo-2,3-diphenylacrylate (16a and 16a')<smiles>CC(CBr)OC(=O)/C(=C(\Br)c1ccccc1)c1ccccc1</smiles><smiles>CC(Br)COC(=O)/C(=C(\Br)c1ccccc1)c1ccccc1</smiles>

The general procedure (Method A) was followed, and 20/1 hexane/ethyl acetate was used as the eluant to afford colorless oil (50.9 mg, 60\%), IR: 3058, 1726, 1171, 692, $556 \mathrm{~cm}^{-1} .{ }^{1} \mathrm{H}$ NMR $(500 \mathrm{MHz}$, Chloroform- $d) \delta 7.27-7.23(\mathrm{~m}, 2 \mathrm{H}), 7.23-7.09(\mathrm{~m}$, $8 \mathrm{H}), 5.32-5.26(\mathrm{~m}, J=6.1 \mathrm{~Hz}, 1 \mathrm{H}), 3.56-3.48(\mathrm{~m}, 1 \mathrm{H}), 1.71(\mathrm{~d}, J=6.7 \mathrm{~Hz}, 1 \mathrm{H}), 1.47$ $(\mathrm{d}, J=6.4 \mathrm{~Hz}, 2 \mathrm{H}) .{ }^{13} \mathrm{C}$ NMR $(125 \mathrm{MHz}$, Chloroform- $d$ ) $\delta 167.0,138.2,136.8,134.7$, 129.8, 129.7, 128.9, 128.9, 128.9, 128.4, 128.3, 128.2, 128.2, 128.1, 71.1, 69.9, 43.9, 34.4, 22.6, 18.4. HRMS: (ESI) calculated for $\mathrm{C}_{18} \mathrm{H}_{17} \mathrm{Br}_{2} \mathrm{O}_{2}[\mathrm{M}+\mathrm{H}]^{+} 422.9590$, found 422.9585 .

1-bromo-3-chloropropan-2-yl (Z)-3-bromo-2,3-diphenylacrylate and 2-bromo-3chloropropyl (Z)-3-bromo-2,3-diphenylacrylate (17a and 17a')<smiles>O=C(OC(CCl)CBr)/C(=C(\Br)c1ccccc1)c1ccccc1</smiles><smiles>O=C(OCC(Br)CCl)/C(=C(\Br)c1ccccc1)c1ccccc1</smiles>

The general procedure (Method A) was followed, and 20/1 hexane/ethyl acetate was used as the eluant to afford colorless oil (52.3 mg, 57\%), IR: 3058, 1730, 1167, 692, $556 \mathrm{~cm}^{-1} .{ }^{1} \mathrm{H}$ NMR $(500 \mathrm{MHz}$, Chloroform- $d$ ) $\delta 7.50-7.48(\mathrm{~m}, 1 \mathrm{H}), 7.45-7.38(\mathrm{~m}$, $1 \mathrm{H}), 7.28-7.24(\mathrm{~m}, 2 \mathrm{H}), 7.21-7.11(\mathrm{~m}, 6 \mathrm{H}), 5.39-5.34(\mathrm{~m}, 1 \mathrm{H}), 3.87(\mathrm{~d}, J=5.2 \mathrm{~Hz}$, 2H), 3.72 - 3.66 (m, 2H). ${ }^{13} \mathrm{C}$ NMR (125 MHz, Chloroform- $d$ ) $\delta 166.6,138.0,136.2$, 134.4, 129.7, 129.5, 129.0, 128.9, 128.8, 128.7, 128.4, 128.4, 128.4, 128.3, 128.1, 124.6, 72.8, 72.4, 43.0, 42.5, 29.7, 29.3. HRMS: (ESI) calculated for $\mathrm{C}_{18} \mathrm{H}_{16} \mathrm{Br}_{2} \mathrm{ClO}_{2}[\mathrm{M}+\mathrm{H}]^{+}$ 456.9200 , found 456.9205 .

\section{3-bromo-2,2-bis(chloromethyl)propyl (Z)-3-bromo-2,3-diphenylacrylate (18a)}<smiles>O=C(OCC(CCl)(CCl)CBr)/C(=C(\Br)c1ccccc1)c1ccccc1</smiles>

The general procedure (Method A) was followed, and 20/1 hexane/ethyl acetate was used as the eluant to afford colorless oil (89.7 mg, 86\%), IR: 2969, 1739, 1180, 709, $548 \mathrm{~cm}^{-1} .{ }^{1} \mathrm{H}$ NMR $(500 \mathrm{MHz}$, Chloroform- $d$ ) $\delta 7.25-7.11(\mathrm{~m}, 8 \mathrm{H}), 7.09-6.99(\mathrm{~m}$, 
2H), $4.36(\mathrm{~s}, 2 \mathrm{H}), 3.62(\mathrm{~s}, 4 \mathrm{H}), 3.48(\mathrm{~s}, 2 \mathrm{H}) .{ }^{13} \mathrm{C}$ NMR (125 MHz, Chloroform- $d$ ) $\delta$ 166.7, 138.2, 136.1, 135.0, 129.7, 129.0, 128.8, 128.5, 128.3, 128.1, 125.6, 63.7, 44.7, 44.2, 32.8. HRMS: (ESI) calculated for $\mathrm{C}_{20} \mathrm{H}_{19} \mathrm{Br}_{2} \mathrm{Cl}_{2} \mathrm{O}_{2}[\mathrm{M}+\mathrm{H}]^{+} 518.9123$, found 518.9125 .

3-bromo-2-(bromomethyl)-2-methylpropyl (19a)<smiles>CC(CBr)(CBr)COC(=O)/C(=C(\Br)c1ccccc1)c1ccccc1</smiles>

(Z)-3-bromo-2,3-diphenylacrylate

The general procedure (Method A) was followed, and 20/1 hexane/ethyl acetate was used as the eluant to afford colorless oil (84.0 mg, 79\%), IR: 2920, 1718, 1186, 714, $556 \mathrm{~cm}^{-1} .{ }^{1} \mathrm{H}$ NMR (500 MHz, Chloroform- $d$ ) $\delta 7.25-7.22(\mathrm{~m}, 2 \mathrm{H}), 7.21-7.15(\mathrm{~m}$, $6 \mathrm{H}), 7.10-7.07(\mathrm{~m}, 2 \mathrm{H}), 4.26(\mathrm{~s}, 2 \mathrm{H}), 3.42(\mathrm{~s}, 4 \mathrm{H}), 1.19(\mathrm{~s}, 3 \mathrm{H}) .{ }^{13} \mathrm{C} \mathrm{NMR}(126 \mathrm{MHz}$, Chloroform- $d$ ) $\delta 167.1,138.2,136.5,135.0,129.7,128.9,128.9,128.4,128.2,128.1$, 125.0, 68.1, 39.4, 38.1, 20.2. HRMS: (ESI) calculated for $\mathrm{C}_{20} \mathrm{H}_{20} \mathrm{Br}_{3} \mathrm{O}_{2}[\mathrm{M}+\mathrm{H}]^{+}$ 528.9008 , found 528.9011 .

\section{3-bromo-2,2-bis(bromomethyl)propyl (Z)-3-bromo-2,3-diphenylacrylate (20a)}<smiles>O=C(OCC(CBr)(CBr)CBr)/C(=C(\Br)c1ccccc1)c1ccccc1</smiles>

The general procedure (Method A) was followed, and 20/1 hexane/ethyl acetate was used as the eluant to afford colorless oil ( $83.0 \mathrm{mg}, 68 \%)$, IR: 2920, 1735, 1177, 696, $603 \mathrm{~cm}^{-1} .{ }^{1} \mathrm{H}$ NMR (500 MHz, Chloroform- $d$ ) $\delta 7.25-7.24(\mathrm{~m}, 2 \mathrm{H}), 7.22-7.17(\mathrm{~m}$, $6 \mathrm{H}), 7.10-7.06(\mathrm{~m}, 2 \mathrm{H}), 4.38$ (s, 2H), 3.49 (s, 6H). ${ }^{13} \mathrm{C}$ NMR (125 MHz, Chloroformd) $\delta 166.6,138.2,136.1,135.0,129.7,129.0,128.9,128.5,128.2,128.1,125.7,64.5$, 43.0, 33.8. HRMS: (ESI) calculated for $\mathrm{C}_{20} \mathrm{H}_{19} \mathrm{Br}_{4} \mathrm{O}_{2}[\mathrm{M}+\mathrm{H}]^{+} 606.8113$, found 606.8108 .

\section{3-bromopropyl (Z)-3-bromo-2,3-diphenylacrylate (21a)}<smiles>O=C(OCCCBr)/C(=C(\Br)c1ccccc1)c1ccccc1</smiles>

The general procedure (Method A) was followed, and 20/1 hexane/ethyl acetate was used as the eluant to afford colorless oil $(71.3 \mathrm{mg}, 84 \%)$, IR: 2962, 1725, 1171, 691, $556 \mathrm{~cm}^{-1} .{ }^{1} \mathrm{H}$ NMR (500 MHz, Chloroform- $d$ ) $\delta 7.26-7.23(\mathrm{~m}, 2 \mathrm{H}), 7.23-7.14(\mathrm{~m}$, $6 \mathrm{H}), 7.11-7.09(\mathrm{~m}, 2 \mathrm{H}), 4.42(\mathrm{t}, J=5.9 \mathrm{~Hz}, 2 \mathrm{H}), 3.47(\mathrm{t}, J=6.5 \mathrm{~Hz}, 2 \mathrm{H}), 2.30-2.25$ (m, 2H). ${ }^{13} \mathrm{C}$ NMR (125 MHz, Chloroform- $d$ ) $\delta 167.7,138.2,136.9,134.8,129.7,128.9$, 128.8, 128.4, 128.2, 128.1, 123.9, 63.3, 31.5, 29.3. HRMS: (ESI) calculated for $\mathrm{C}_{18} \mathrm{H}_{17} \mathrm{Br}_{2} \mathrm{O}_{2}[\mathrm{M}+\mathrm{H}]^{+}$422.9590, found 422.9587 . 


\section{5-bromopentyl (Z)-3-bromo-2,3-diphenylacrylate (22a)}<smiles>O=C(OCCCCCBr)/C(=C(\Br)c1ccccc1)c1ccccc1</smiles>

The general procedure (Method A) was followed, and 20/1 hexane/ethyl acetate was used as the eluant to afford colorless oil (42.6 mg, 47\%), IR: 2938, 1723, 1172, 692, $557 \mathrm{~cm}^{-1} .{ }^{1} \mathrm{H}$ NMR (500 MHz, Chloroform- $d$ ) $\delta 7.25-7.24(\mathrm{~m}, 2 \mathrm{H}), 7.20-7.16(\mathrm{~m}$, $6 \mathrm{H}), 7.12-7.10(\mathrm{~m}, 2 \mathrm{H}), 4.30(\mathrm{t}, J=6.5 \mathrm{~Hz}, 2 \mathrm{H}), 3.38(\mathrm{t}, J=6.7 \mathrm{~Hz}, 2 \mathrm{H}), 1.90-1.85$ $(\mathrm{m}, 2 \mathrm{H}), 1.79-1.73(\mathrm{~m}, 2 \mathrm{H}), 1.57-1.51(\mathrm{~m}, 2 \mathrm{H}) .{ }^{13} \mathrm{C}$ NMR $(125 \mathrm{MHz}$, Chloroformd) $\delta 167.9,138.3,137.2,134.9,129.7,128.8,128.3,128.1,128.1,123.6,65.4,33.3$, 32.2, 27.6, 24.6. HRMS: (ESI) calculated for $\mathrm{C}_{20} \mathrm{H}_{21} \mathrm{Br}_{2} \mathrm{O}_{2}[\mathrm{M}+\mathrm{H}]^{+} 450.9903$, found 450.9899 .

\section{6-bromohexyl (Z)-3-bromo-2,3-diphenylacrylate (23a)}<smiles>O=C(OCCCCCCBr)/C(=C(\Br)c1ccccc1)c1ccccc1</smiles>

The general procedure (Method A) was followed, and 20/1 hexane/ethyl acetate was used as the eluant to afford colorless oil (37.3 mg, 40\%), IR: 2934, 1723, 1173, 692, $557 \mathrm{~cm}^{-1} .{ }^{1} \mathrm{H}$ NMR $(500 \mathrm{MHz}$, Chloroform- $d) \delta 7.26-7.22(\mathrm{~m}, 2 \mathrm{H}), 7.20-7.14(\mathrm{~m}$, $6 \mathrm{H}), 7.12-7.09(\mathrm{~m}, 2 \mathrm{H}), 4.29(\mathrm{t}, J=6.6 \mathrm{~Hz}, 2 \mathrm{H}), 3.38(\mathrm{t}, J=6.8 \mathrm{~Hz}, 2 \mathrm{H}), 1.87-1.81$ $(\mathrm{m}, 2 \mathrm{H}), 1.78-1.72(\mathrm{~m}, 2 \mathrm{H}), 1.48-1.38(\mathrm{~m}, 4 \mathrm{H}) .{ }^{13} \mathrm{C}$ NMR $(125 \mathrm{MHz}$, Chloroformd) $\delta 167.9,138.3,137.2,135.0,129.7,128.8,128.8,128.3,128.1,123.5,65.6,33.5$, 32.6, 28.2, 27.6, 25.1. HRMS: (ESI) calculated for $\mathrm{C}_{21} \mathrm{H}_{23} \mathrm{Br}_{2} \mathrm{O}_{2}[\mathrm{M}+\mathrm{H}]^{+} 465.0059$, found 465.0058 .

\section{2-chlorocyclohexyl (Z)-3-chloro-2,3-diphenylacrylate (1b)}<smiles>O=C(O[C@H]1CCCC[C@H]1Cl)/C(=C(\Cl)c1ccccc1)c1ccccc1</smiles>

The general procedure (Method B) was followed, and 20/1 hexane/ethyl acetate was used as the eluant to afford colorless oil (66.9 mg, 89\%), IR: 2940, 1729, 1233, 692, $563 \mathrm{~cm}^{-1} .{ }^{1} \mathrm{H}$ NMR (500 MHz, Chloroform- $d$ ) $\delta 7.31-7.26(\mathrm{~m}, 2 \mathrm{H}), 7.23-7.13(\mathrm{~m}$, $8 \mathrm{H}), 5.06-5.01(\mathrm{~m}, 1 \mathrm{H}), 3.99-3.93(\mathrm{~m}, 1 \mathrm{H}), 2.26-2.23(\mathrm{~m}, 2 \mathrm{H}), 1.80-1.71(\mathrm{~m}, 3 \mathrm{H})$, $1.53-1.42(\mathrm{~m}, 2 \mathrm{H}), 1.35-1.29(\mathrm{~m}, 1 \mathrm{H}) .{ }^{13} \mathrm{C}$ NMR $(125 \mathrm{MHz}$, Chloroform- $d$ ) $\delta 166.4$, 136.7, 134.4, 133.7, 133.2, 129.6, 129.2, 129.0, 128.3, 128.1, 128.0, 60.0, 34.6, 30.1, 24.2, 23.0. HRMS: (ESI) calculated for $\mathrm{C}_{21} \mathrm{H}_{21} \mathrm{Cl}_{2} \mathrm{O}_{2}[\mathrm{M}+\mathrm{H}]^{+}$375.0913, found 375.0915 .

\section{3-chloropropyl (Z)-3-chloro-2,3-diphenylacrylate (2b)}


<smiles>O=C(OCCCCl)/C(=C(/Cl)c1ccccc1)c1ccccc1</smiles>

The general procedure (Method B) was followed, and 20/1 hexane/ethyl acetate was used as the eluant to afford colorless oil (55.7 mg, 83\%), IR: 2965, 1725, 1173, 692, $567 \mathrm{~cm}^{-1} .{ }^{1} \mathrm{H}$ NMR (400 MHz, Chloroform- $d$ ) $\delta 7.25-7.23(\mathrm{~m}, 2 \mathrm{H}), 7.24-7.15(\mathrm{~m}$, $6 \mathrm{H}), 7.15-7.06(\mathrm{~m}, 2 \mathrm{H}), 4.43(\mathrm{t}, J=6.0 \mathrm{~Hz}, 2 \mathrm{H}), 3.59(\mathrm{t}, J=6.4 \mathrm{~Hz}, 2 \mathrm{H}), 2.20-2.14$ $(\mathrm{m}, 2 \mathrm{H}) .{ }^{13} \mathrm{C}$ NMR $(125 \mathrm{MHz}$, Chloroform- $d) \delta 167.2,136.5,134.4,133.6,133.4,129.6$, 129.1, 129.0, 128.4, 128.2, 128.1, 62.2, 41.1, 31.5. HRMS: (ESI) calculated for $\mathrm{C}_{18} \mathrm{H}_{17} \mathrm{Cl}_{2} \mathrm{O}_{2}[\mathrm{M}+\mathrm{H}]^{+}$335.0600, found 335.0605.

\section{4-chlorobutyl (Z)-3-chloro-2,3-diphenylacrylate (3b)}<smiles>O=C(OCCCCCl)/C(=C(\Cl)c1ccccc1)c1ccccc1</smiles>

The general procedure (Method B) was followed, and 20/1 hexane/ethyl acetate was used as the eluant to afford colorless oil (60.1 mg, 86\%), IR: 2959, 1724, 1174, 722, $692 \mathrm{~cm}^{-1} .{ }^{1} \mathrm{H}$ NMR $(400 \mathrm{MHz}$, Chloroform- $d) \delta 7.33-7.22(\mathrm{~m}, 8 \mathrm{H}), 7.18-7.15(\mathrm{~m}$, $2 \mathrm{H}), 4.37(\mathrm{t}, J=4.8 \mathrm{~Hz}, 2 \mathrm{H}), 3.64-3.56(\mathrm{~m}, 2 \mathrm{H}), 1.98-1.88(\mathrm{~m}, 4 \mathrm{H}) .{ }^{13} \mathrm{C}$ NMR $(125$ $\mathrm{MHz}$, Chloroform- $d$ ) $\delta 136.6,134.5,133.6,133.4,129.6,129.0,128.4,128.1,128.0$, 64.7, 44.3, 29.0, 25.9. HRMS: (ESI) calculated for $\mathrm{C}_{19} \mathrm{H}_{19} \mathrm{Cl}_{2} \mathrm{O}_{2}[\mathrm{M}+\mathrm{H}]^{+}$349.0757, found 335.0751 .

\section{4-chlorobutyl (Z)-3-chloro-2,3-bis(4-fluorophenyl)acrylate (4b)}<smiles>O=C(OCCCCCl)/C(=C(\Cl)c1ccc(F)cc1)c1ccc(F)cc1</smiles>

The general procedure (Method B) was followed, and 20/1 hexane/ethyl acetate was used as the eluant to afford colorless oil (70.2 mg, 91\%), IR: 2960, 1725, 1504, 1158, $558 \mathrm{~cm}^{-1} .{ }^{1} \mathrm{H}$ NMR $(500 \mathrm{MHz}$, Chloroform- $d$ ) $\delta 7.25-7.21(\mathrm{~m}, 2 \mathrm{H}), 7.11-7.07(\mathrm{~m}$, $2 \mathrm{H}), 6.93-6.88(\mathrm{~m}, 4 \mathrm{H}), 4.32(\mathrm{t}, J=5.8 \mathrm{~Hz}, 2 \mathrm{H}), 3.55(\mathrm{t}, J=5.9 \mathrm{~Hz}, 2 \mathrm{H}), 1.92-1.81$ $(\mathrm{m}, 4 \mathrm{H}) .{ }^{13} \mathrm{C}$ NMR $(125 \mathrm{MHz}$, Chloroform- $d) \delta 167.0,163.6(\mathrm{~J}=45 \mathrm{~Hz}), 161.6(\mathrm{~J}=45$ $\mathrm{Hz}), 132.6(J=41.2 \mathrm{~Hz}), 132.6(J=41.2 \mathrm{~Hz}), 131.5(J=7.5 \mathrm{~Hz}), 130.9(J=8.8 \mathrm{~Hz})$, $130.4(J=3.8 \mathrm{~Hz}), 115.6(J=42.5 \mathrm{~Hz}), 115.4(J=42.5 \mathrm{~Hz}), 64.9,44.2,29.0,25.9 .{ }^{19} \mathrm{~F}$ NMR (471 MHz, Chloroform- $d$ ) $\delta-110.5,-112.2$. HRMS: (ESI) calculated for $\mathrm{C}_{19} \mathrm{H}_{17} \mathrm{Cl}_{2} \mathrm{~F}_{2} \mathrm{O}_{2}[\mathrm{M}+\mathrm{H}]^{+}$385.0568, found 385.0570.

\section{4-chlorobutyl (Z)-2,3-bis(4-bromophenyl)-3-chloroacrylate (5b)}


<smiles>O=C(OCCCCCl)/C(=C(\Cl)c1ccc(Br)cc1)c1ccc(Br)cc1</smiles>

The general procedure (Method B) was followed, and 20/1 hexane/ethyl acetate was used as the eluant to afford colorless oil (78.1 mg, 77\%), IR: 2958, 1724, 1175, 740, $412 \mathrm{~cm}^{-1} .{ }^{1} \mathrm{H}$ NMR $(400 \mathrm{MHz}$, Chloroform- $d$ ) $\delta 7.43-7.39(\mathrm{~m}, 4 \mathrm{H}), 7.19-7.15(\mathrm{~m}, 2 \mathrm{H})$, $7.05-7.02(\mathrm{~m}, 2 \mathrm{H}), 4.38-4.34(\mathrm{~m}, 2 \mathrm{H}), 3.63-3.58(\mathrm{~m}, 2 \mathrm{H}), 1.96-1.90(\mathrm{~m}, 4 \mathrm{H}) .{ }^{13} \mathrm{C}$ NMR (125 MHz, Chloroform- $d$ ) $\delta$ 166.6, 135.1, 133.0, 131.9, 131.5, 131.0, 130.5, 123.7, 122.8, 65.0, 44.2, 29.0, 25.9. HRMS: (ESI) calculated for $\mathrm{C}_{19} \mathrm{H}_{17} \mathrm{Br}_{2} \mathrm{Cl}_{2} \mathrm{O}_{2}$ $[\mathrm{M}+\mathrm{H}]^{+}$504.8967, found 504.8961.

\section{4-chlorobutyl (Z)-3-chloro-2,3-di-p-tolylacrylate (6b)}<smiles>Cc1ccc(C(Cl)=C(C(=O)OCCCCCl)c2ccc(C)cc2)cc1</smiles>

The general procedure (Method B) was followed, and 20/1 hexane/ethyl acetate was used as the eluant to afford colorless oil (59.7 mg, 79\%), IR: 2958, 1724, 1171, 785, $500 \mathrm{~cm}^{-1}{ }^{1}{ }^{\mathrm{H}} \mathrm{NMR}(400 \mathrm{MHz}$, Chloroform- $d$ ) $\delta 7.24-7.19(\mathrm{~m}, 2 \mathrm{H}), 7.06-7.04(\mathrm{~m}$, $6 \mathrm{H}), 4.42-4.29(\mathrm{~m}, 2 \mathrm{H}), 3.62-3.57(\mathrm{~m}, 2 \mathrm{H}), 2.35-2.31(\mathrm{~m}, 6 \mathrm{H}), 1.96-1.90(\mathrm{~m}$, $4 \mathrm{H}) .{ }^{13} \mathrm{C}$ NMR $(125 \mathrm{MHz}$, Chloroform- $d$ ) $\delta$ 167.6, 139.0, 137.9, 133.7, 131.7, 129.4, 129.1, 128.7, 64.6, 44.3, 29.0, 25.9, 21.1. HRMS: (ESI) calculated for $\mathrm{C}_{21} \mathrm{H}_{23} \mathrm{Cl}_{2} \mathrm{O}_{2}$ $[\mathrm{M}+\mathrm{H}]^{+} 377.1070$, found 377.1072 .

\section{4-chlorobutyl (Z)-2,3-bis(4-(tert-butyl)phenyl)-3-chloroacrylate (7b)}<smiles>CC(C)(C)c1ccc(C(Cl)=C(C(=O)OCCCCCl)c2ccc(C(C)(C)C)cc2)cc1</smiles>

The general procedure (Method B) was followed, and 20/1 hexane/ethyl acetate was used as the eluant to afford colorless oil (70.2 mg, 79\%), IR: 2961, 1727, 1177, 820, $560 \mathrm{~cm}^{-1} .{ }^{1} \mathrm{H}$ NMR (400 MHz, Chloroform- $d$ ) $\delta 7.29-7.23(\mathrm{~m}, 6 \mathrm{H}), 7.12-7.06(\mathrm{~m}$, $2 \mathrm{H}), 4.40-4.36(\mathrm{~m}, 2 \mathrm{H}), 3.62-3.58(\mathrm{~m}, 2 \mathrm{H}), 1.96-1.92(\mathrm{~m}, 4 \mathrm{H}), 1.34-1.30(\mathrm{~m}$, $18 \mathrm{H}) .{ }^{13} \mathrm{C}$ NMR $(125 \mathrm{MHz}$, Chloroform- $d$ ) $\delta 167.7,152.2,151.1,133.0,129.3,128.6$, 125.3, 124.9, 64.6, 44.3, 34.6, 34.5, 31.1, 31.1, 29.1, 26.0. HRMS: (ESI) calculated for $\mathrm{C}_{27} \mathrm{H}_{35} \mathrm{Cl}_{2} \mathrm{O}_{2}[\mathrm{M}+\mathrm{H}]^{+}$461.2009, found 461.2008. 


\section{4-chlorobutyl (Z)-2,3-bis(3-bromophenyl)-3-chloroacrylate (8b)}<smiles>O=C(OCCCCCl)/C(=C(\Cl)c1cccc(Br)c1)c1cccc(Br)c1</smiles>

The general procedure (Method B) was followed, and 20/1 hexane/ethyl acetate was used as the eluant to afford colorless oil (70.1 mg, 75\%), IR: 2958, 1727, 1179, 686, $433 \mathrm{~cm}^{-1} .{ }^{1} \mathrm{H}$ NMR (400 MHz, Chloroform- $d$ ) $\delta 7.48-7.47(\mathrm{~m}, 1 \mathrm{H}), 7.44-7.33(\mathrm{~m}$, $2 \mathrm{H}), 7.32-7.31(\mathrm{~m}, 1 \mathrm{H}), 7.12-7.04(\mathrm{~m}, 3 \mathrm{H}), 7.03-6.99(\mathrm{~m}, 1 \mathrm{H}), 4.32(\mathrm{t}, J=5.8 \mathrm{~Hz}$, 2H), $3.58-3.52(\mathrm{~m}, 2 \mathrm{H}), 1.90-1.86(\mathrm{~m}, 4 \mathrm{H}) .{ }^{13} \mathrm{C}$ NMR $(125 \mathrm{MHz}$, Chloroform- $d) \delta$ 166.4, 138.0, 136.0, 133.1, 133.0, 132.5, 132.4, 131.7, 131.6, 130.1, 129.7, 128.1, 127.8, 122.5, 122.2, 65.1, 44.3, 29.0, 25.9. HRMS: (ESI) calculated for $\mathrm{C}_{19} \mathrm{H}_{17} \mathrm{Cl}_{4} \mathrm{O}_{2}[\mathrm{M}+\mathrm{H}]^{+}$ 416.9977, found 416.9976 .

\section{4-chlorobutyl (Z)-3-chloro-2-ethylpent-2-enoate (9b)}<smiles>CCC(Cl)=C(CC)C(=O)OCCCCCl</smiles>

The general procedure (Method B) was followed, and 20/1 hexane/ethyl acetate was used as the eluant to afford colorless oil (37.5 mg, 74\%), IR: 2971, 1723, 1140, 754, $447 \mathrm{~cm}^{-1} .{ }^{1} \mathrm{H}$ NMR $(500 \mathrm{MHz}$, Chloroform- $d$ ) $\delta 4.24(\mathrm{t}, J=6.3 \mathrm{~Hz}, 2 \mathrm{H}), 3.45(\mathrm{t}, J=6.6$ $\mathrm{Hz}, 2 \mathrm{H}), 2.54(\mathrm{q}, J=7.4 \mathrm{~Hz}, 2 \mathrm{H}), 2.34(\mathrm{q}, J=7.6 \mathrm{~Hz}, 2 \mathrm{H}), 2.03-1.98(\mathrm{~m}, 2 \mathrm{H}), 1.91-$ $1.84(\mathrm{~m}, 2 \mathrm{H}), 1.16(\mathrm{t}, J=7.4 \mathrm{~Hz}, 3 \mathrm{H}), 1.07(\mathrm{t}, J=7.6 \mathrm{~Hz}, 3 \mathrm{H}) .{ }^{13} \mathrm{C} \mathrm{NMR}(125 \mathrm{MHz}$, Chloroform- $d$ ) $\delta 168.6,135.3,127.9,63.9,32.9,30.5,29.3,27.2,24.6,13.0,13.0$. HRMS: (ESI) calculated for $\mathrm{C}_{11} \mathrm{H}_{19} \mathrm{Cl}_{2} \mathrm{O}_{2}[\mathrm{M}+\mathrm{H}]^{+}$253.0757, found 253.0759.

\section{4-chlorobutyl (Z)-2-butyl-3-chlorohept-2-enoate (10b)}<smiles>CC(C)(C)C(Cl)=C(C(=O)O)C(=O)OCCCCCl</smiles>

The general procedure (Method B) was followed, and 20/1 hexane/ethyl acetate was used as the eluant to afford colorless oil (48.3 mg, 78\%), IR: 2958, 1725, 1272, 1148, $652 \mathrm{~cm}^{-1} .{ }^{1} \mathrm{H}$ NMR $(500 \mathrm{MHz}$, Chloroform- $d) \delta 4.23(\mathrm{t}, J=5.9 \mathrm{~Hz}, 2 \mathrm{H}), 3.58(\mathrm{t}, J=6.2$ $\mathrm{Hz}, 2 \mathrm{H}), 2.43-2.37(\mathrm{~m}, 2 \mathrm{H}), 2.35-2.28(\mathrm{~m}, 2 \mathrm{H}), 1.93-1.83(\mathrm{~m}, 4 \mathrm{H}), 1.62-1.55(\mathrm{~m}$, 2H), $1.42-1.30(\mathrm{~m}, 8 \mathrm{H}), 0.96-0.89(\mathrm{~m}, 6 \mathrm{H}) .{ }^{13} \mathrm{C}$ NMR $(125 \mathrm{MHz}$, Chloroform- $d) \delta$ 168.2, 135.4, 131.3, 69.9, 63.9, 44.3, 34.9, 30.6, 29.5, 29.2, 27.0, 26.0, 22.2, 22.0, 13.8. HRMS: (ESI) calculated for $\mathrm{C}_{15} \mathrm{H}_{27} \mathrm{Cl}_{2} \mathrm{O}_{2}[\mathrm{M}+\mathrm{H}]^{+} 309.1383$, found 309.1383.

\section{4-chlorobutyl (Z)-3-chloro-3-(4-methoxyphenyl)acrylate (11b)}


<smiles>COc1ccc(/C(Cl)=C/C(=O)OCCCCCl)cc1</smiles>

The general procedure (Method B) was followed, and 20/1 hexane/ethyl acetate was used as the eluant to afford colorless oil (39.5 mg, 65\%), IR: 2959, 1725, 1176, 815, $579 \mathrm{~cm}^{-1} .{ }^{1} \mathrm{H}$ NMR $(500 \mathrm{MHz}$, Chloroform- $d) \delta 7.27-7.23(\mathrm{~m}, 2 \mathrm{H}), 6.90-6.86(\mathrm{~m}$, $2 \mathrm{H}), 6.53(\mathrm{~s}, 1 \mathrm{H}), 4.35-4.32(\mathrm{~m}, 2 \mathrm{H}), 3.81(\mathrm{~s}, 3 \mathrm{H}), 3.57-3.55(\mathrm{~m}, 2 \mathrm{H}), 1.91-1.87$ (m, 4H). ${ }^{13} \mathrm{C}$ NMR (125 MHz, Chloroform- $d$ ) $\delta 166.1,160.1,137.2,128.0,126.6,119.4$, 114.2, 64.6, 55.3, 44.3, 29.1, 26.0. HRMS: (ESI) calculated for $\mathrm{C}_{14} \mathrm{H}_{17} \mathrm{Cl}_{2} \mathrm{O}_{3}[\mathrm{M}+\mathrm{H}]^{+}$ 303.0549 , found 303.0550 .

\section{4-chlorobutyl (Z)-3-(4-bromophenyl)-3-chloroacrylate (12b)}<smiles>O=C(/C=C(\Cl)c1ccc(Br)cc1)OCCCCCl</smiles>

The general procedure (Method B) was followed, and 20/1 hexane/ethyl acetate was used as the eluant to afford colorless oil (42.3 mg, 60\%), IR: 2959, 1726, 1195, 807, $405 \mathrm{~cm}^{-1} .{ }^{1} \mathrm{H}$ NMR (400 MHz, Chloroform- $d$ ) $\delta 7.56-7.45(\mathrm{~m}, 2 \mathrm{H}), 7.23-7.16(\mathrm{~m}$, $2 \mathrm{H}), 6.64(\mathrm{~s}, 1 \mathrm{H}), 4.37-4.31(\mathrm{~m}, 2 \mathrm{H}), 3.63-3.58(\mathrm{~m}, 2 \mathrm{H}), 1.90-1.87(\mathrm{~m}, 4 \mathrm{H}) .{ }^{13} \mathrm{C}$ NMR (125 MHz, Chloroform- $d$ ) $\delta$ 165.4, 136.5, 133.2, 131.9, 128.4, 123.1, 122.5, 64.8, 44.3, 29.7, 29.0, 25.9. HRMS: (ESI) calculated for $\mathrm{C}_{13} \mathrm{H}_{14} \mathrm{BrCl}_{2} \mathrm{O}_{2}[\mathrm{M}+\mathrm{H}]^{+} 350.9549$, found 350.9551 .

\section{4-chlorobutyl (Z)-3-chloro-3-(naphthalen-2-yl)acrylate (13b)}<smiles>O=C(/C=C(\Cl)c1ccc2ccccc2c1)OCCCCCl</smiles>

The general procedure (Method B) was followed, and 20/1 hexane/ethyl acetate was used as the eluant to afford colorless oil (55.6 mg, 86\%), IR: 2959, 1725, 1177, 809, $474 \mathrm{~cm}^{-1} .{ }^{1} \mathrm{H}$ NMR $(500 \mathrm{MHz}$, Chloroform- $d) \delta 7.83-7.80(\mathrm{~m}, 3 \mathrm{H}), 7.77-7.76(\mathrm{~m}, 1 \mathrm{H})$, $7.51-7.49(\mathrm{~m}, 2 \mathrm{H}), 7.45-7.42(\mathrm{~m}, 1 \mathrm{H}), 6.75(\mathrm{~s}, 1 \mathrm{H}), 4.40-4.36(\mathrm{~m}, 2 \mathrm{H}), 3.57-3.54$ (m, 2H), $1.92-1.87$ (m, 4H). ${ }^{13} \mathrm{C}$ NMR (125 MHz, Chloroform- $d$ ) $\delta 165.9,137.8,133.2$, 133.1, 131.5, 128.5, 128.2, 127.6, 126.8, 126.7, 126.2, 124.0, 121.6, 64.7, 44.3, 29.1, 26.0. HRMS: (ESI) calculated for $\mathrm{C}_{17} \mathrm{H}_{17} \mathrm{Cl}_{2} \mathrm{O}_{2}[\mathrm{M}+\mathrm{H}]^{+}$323.0600, found 323.0597.

4-chlorobutyl (Z)-3-chloro-2-methyl-3-phenylacrylate and 4-chlorobutyl (Z)-3chloro-2-phenylbut-2-enoate (14b and 14b')<smiles>C/C(C(=O)OCCCCCl)=C(/Cl)c1ccccc1</smiles><smiles>C/C(Cl)=C(\C(=O)OCCCCCl)c1ccccc1</smiles> 
The general procedure (Method B) was followed, and 20/1 hexane/ethyl acetate was used as the eluant to afford colorless oil (47.2 mg, 82\%), IR (14b): 2959, 1725, 1205, $698,599 \mathrm{~cm}^{-1} .{ }^{1} \mathrm{H}$ NMR $(500 \mathrm{MHz}$, Chloroform- $d, \mathbf{1 4 b}) \delta 7.42-7.34(\mathrm{~m}, 5 \mathrm{H}), 4.31(\mathrm{t}$, $J=5.8 \mathrm{~Hz}, 2 \mathrm{H}), 3.61(\mathrm{t}, J=5.9 \mathrm{~Hz}, 2 \mathrm{H}), 1.98-1.88(\mathrm{~m}, 7 \mathrm{H}) .{ }^{13} \mathrm{C} \mathrm{NMR}(125 \mathrm{MHz}$, Chloroform- $d$, 14b) $\delta 168.2,137.3,132.4,129.0,128.6,128.3,127.8,64.3,44.3,29.1$, 26.0, 18.1. HRMS: (ESI, 14b) calculated for $\mathrm{C}_{14} \mathrm{H}_{17} \mathrm{Cl}_{2} \mathrm{O}_{2}[\mathrm{M}+\mathrm{H}]^{+} 287.0600$, found 287.0599. IR (14b'): 2924, 1722, 1209, 691, 591 $\mathrm{cm}^{-1} .{ }^{1} \mathrm{H}$ NMR (500 MHz, Chloroform- $d$, 14b') $\delta 7.39-7.33(\mathrm{~m}, 3 \mathrm{H}), 7.29-7.26(\mathrm{~m}, 2 \mathrm{H}), 4.22(\mathrm{t}, J=4.2 \mathrm{~Hz}$, 2H), $3.53-3.47(\mathrm{~m}, 2 \mathrm{H}), 2.13(\mathrm{~s}, 3 \mathrm{H}), 1.82-1.80(\mathrm{~m}, 4 \mathrm{H}) .{ }^{13} \mathrm{C}$ NMR $(125 \mathrm{MHz}$, Chloroform- $d$, 14b') $\delta 166.7,134.8,134.3,132.7,128.7,128.6,128.3,64.4,44.3,29.0$, 25.9, 23.7. HRMS: (ESI, 14b') calculated for $\mathrm{C}_{14} \mathrm{H}_{17} \mathrm{Cl}_{2} \mathrm{O}_{2}[\mathrm{M}+\mathrm{H}]^{+} 287.0600$, found 287.0599 .

4-chlorobutyl (Z)-2-(chloro(phenyl)methylene)-3-methylbutanoate and 4chlorobutyl (Z)-3-chloro-4-methyl-2-phenylpent-2-enoate (15b and 15b')<smiles>CCCC(C(=O)OCCCCCl)=C(Cl)c1ccccc1</smiles><smiles>CCCC(Cl)=C(C(=O)OCCCCCl)c1ccccc1</smiles>

The general procedure (Method B) was followed, and 20/1 hexane/ethyl acetate was used as the eluant to afford colorless oil (42.9 mg, 68\%), IR: 2965, 1724, 1265, 697, $592 \mathrm{~cm}^{-1} .{ }^{1} \mathrm{H}$ NMR $(500 \mathrm{MHz}$, Chloroform- $d$ ) $\delta 7.41-7.34(\mathrm{~m}, 4 \mathrm{H}), 7.29-7.28(\mathrm{~m}, 1 \mathrm{H})$, $4.32(\mathrm{t}, J=5.8 \mathrm{~Hz}, 1 \mathrm{H}), 4.20(\mathrm{t}, J=5.5 \mathrm{~Hz}, 1 \mathrm{H}), 3.61(\mathrm{t}, J=5.9 \mathrm{~Hz}, 1 \mathrm{H}), 3.49(\mathrm{t}, J=$ $5.7 \mathrm{~Hz}, 1 \mathrm{H}), 2.73-2.65(\mathrm{~m}, 1 \mathrm{H}), 1.99-1.89(\mathrm{~m}, 3 \mathrm{H}), 1.82-1.78(\mathrm{~m}, 1 \mathrm{H}), 1.10-1.06$ $(\mathrm{m}, 6 \mathrm{H}) .{ }^{13} \mathrm{C}$ NMR $(125 \mathrm{MHz}$, Chloroform- $d$ ) $\delta 167.2,139.4,136.9,128.9,128.6,128.5$, 128.5, 128.4, 128.2, 64.3, 64.2, 44.3, 44.3, 32.2, 31.0, 29.2, 29.0, 26.1, 25.9, 21.3, 20.4.HRMS: (ESI) calculated for $\mathrm{C}_{16} \mathrm{H}_{21} \mathrm{Cl}_{2} \mathrm{O}_{2}[\mathrm{M}+\mathrm{H}]^{+} 315.0913$, found 315.0908 .

\section{5-chloropentyl (Z)-3-chloro-2,3-diphenylacrylate (16b)}<smiles>O=C(OCCCCCCl)/C(=C(/Cl)c1ccccc1)c1ccccc1</smiles>

The general procedure (Method B) was followed, and 20/1 hexane/ethyl acetate was used as the eluant to afford colorless oil (37.8 mg, 52\%), IR: 2955, 1723, 1186, 722, $693 \mathrm{~cm}^{-1} .{ }^{1} \mathrm{H}$ NMR (500 MHz, Chloroform- $d$ ) $\delta 7.27-7.25(\mathrm{~m}, 2 \mathrm{H}), 7.23-7.17(\mathrm{~m}$, $6 \mathrm{H}), 7.13-7.11(\mathrm{~m}, 2 \mathrm{H}), 4.29(\mathrm{t}, J=6.5 \mathrm{~Hz}, 2 \mathrm{H}), 3.51(\mathrm{t}, J=6.6 \mathrm{~Hz}, 2 \mathrm{H}), 1.82-1.72$ $(\mathrm{m}, 4 \mathrm{H}), 1.56-1.47(\mathrm{~m}, 2 \mathrm{H}) .{ }^{13} \mathrm{C}$ NMR $(125 \mathrm{MHz}$, Chloroform- $d$ ) $\delta 167.3,136.6,134.6$, 133.7, 133.2, 129.5, 129.0, 129.0, 128.4, 128.1, 128.0, 65.3, 44.6, 32.0, 27.8, 23.3. HRMS: (ESI) calculated for $\mathrm{C}_{20} \mathrm{H}_{21} \mathrm{Cl}_{2} \mathrm{O}_{2}[\mathrm{M}+\mathrm{H}]^{+} 363.0913$, found 363.0916.

6-chlorohexyl (Z)-3-chloro-2,3-diphenylacrylate (17b) 
<smiles>O=C(OCCCCCCCl)/C(=C(/Cl)c1ccccc1)c1ccccc1</smiles>

The general procedure (Method B) was followed, and 20/1 hexane/ethyl acetate was used as the eluant to afford colorless oil (32.5 mg, 43\%), IR: 2936, 1724, 1175, 722, $693 \mathrm{~cm}^{-1} .{ }^{1} \mathrm{H}$ NMR (400 MHz, Chloroform- $d$ ) $\delta 7.26-7.14(\mathrm{~m}, 8 \mathrm{H}), 7.13-7.07(\mathrm{~m}$, 2H), $4.27(\mathrm{t}, J=6.5 \mathrm{~Hz}, 2 \mathrm{H}), 3.49(\mathrm{t}, J=6.6 \mathrm{~Hz}, 2 \mathrm{H}), 1.78-1.69(\mathrm{~m}, 4 \mathrm{H}), 1.47-1.35$ $(\mathrm{m}, 4 \mathrm{H}) .{ }^{13} \mathrm{C}$ NMR $(125 \mathrm{MHz}$, Chloroform- $d$ ) $\delta 167.4,136.6,134.6,133.8,133.1,129.6$, 129.0, 129.0, 128.4, 128.1, 128.0, 65.5, 44.8, 32.4, 28.3, 26.4, 25.2. HRMS: (ESI) calculated for $\mathrm{C}_{21} \mathrm{H}_{23} \mathrm{Cl}_{2} \mathrm{O}_{2}[\mathrm{M}+\mathrm{H}]^{+}$377.1070, found 377.1075.

\section{4-iodobutyl (Z)-3-iodo-2,3-diphenylacrylate (1c)}<smiles>O=C(OCCCCI)/C(=C(\I)c1ccccc1)c1ccccc1</smiles>

The general procedure (Method C) was followed, and 20/1 hexane/ethyl acetate was used as the eluant to afford colorless oil (47.9 mg, 45\%), IR: 2915, 1722, 1221, 690, $548 \mathrm{~cm}^{-1} .{ }^{1} \mathrm{H}$ NMR (400 MHz, Chloroform- $d$ ) $\delta 7.30-7.29(\mathrm{~m}, 2 \mathrm{H}), 7.24-7.13(\mathrm{~m}$, $8 \mathrm{H}), 7.14-7.08(\mathrm{~m}, 2 \mathrm{H}), 4.34(\mathrm{t}, J=6.1 \mathrm{~Hz}, 2 \mathrm{H}), 3.23(\mathrm{t}, J=6.7 \mathrm{~Hz}, 2 \mathrm{H}), 2.02-1.95$ $(\mathrm{m}, 2 \mathrm{H}), 1.93$ - $1.86(\mathrm{~m}, 2 \mathrm{H}) .{ }^{13} \mathrm{C}$ NMR $(125 \mathrm{MHz}$, Chloroform- $d$ ) $\delta$ 168.7, 143.6, 141.9, 135.2, 129.4, 128.8, 128.3, 128.2, 128.0, 100.5, 64.6, 29.9, 29.4, 5.7. HRMS: (ESI) calculated for $\mathrm{C}_{19} \mathrm{H}_{19} \mathrm{I}_{2} \mathrm{O}_{2}[\mathrm{M}+\mathrm{H}]^{+} 532.9469$, found 532.9475 .

\section{4-iodobutyl (Z)-2,3-bis(4-(tert-butyl)phenyl)-3-iodoacrylate (2c)}<smiles>CC(C)(C)c1ccc(C(C(=O)OCCCCI)=C(I)c2ccc(Br)cc2)cc1</smiles>

The general procedure (Method C) was followed, and 20/1 hexane/ethyl acetate was used as the eluant to afford colorless oil (64.5 mg, 50\%), IR: 2960, 1723, 1171, 837, $557 \mathrm{~cm}^{-1} .{ }^{1} \mathrm{H}$ NMR $(500 \mathrm{MHz}$, Chloroform- $d) \delta 7.19-7.11(\mathrm{~m}, 6 \mathrm{H}), 6.98(\mathrm{~d}, J=8.4$ $\mathrm{Hz}, 2 \mathrm{H}), 4.30(\mathrm{t}, J=6.2 \mathrm{~Hz}, 2 \mathrm{H}), 3.19(\mathrm{t}, J=6.8 \mathrm{~Hz}, 2 \mathrm{H}), 1.99-1.93(\mathrm{~m}, 2 \mathrm{H}), 1.90-$ $1.83(\mathrm{~m}, 2 \mathrm{H}), 1.25$ (s, 9H), 1.22 (s, 9H). ${ }^{13} \mathrm{C}$ NMR (125 MHz, Chloroform- $d$ ) $\delta 169.0$, 151.4, 150.9, 143.1, 139.1, 132.2, 129.1, 128.4, 125.0, 124.8, 100.2, 64.4, 34.5, 34.5, 31.1, 31.1, 30.0, 29.4, 5.7. HRMS: (ESI) calculated for $\mathrm{C}_{27} \mathrm{H}_{35} \mathrm{I}_{2} \mathrm{O}_{2}[\mathrm{M}+\mathrm{H}]^{+}$645.0721, found 645.0726 .

\section{4-iodobutyl (Z)-2,3-bis(4-fluorophenyl)-3-iodoacrylate (3c)}


<smiles>O=C(OCCCCI)/C(=C(\I)c1ccc(F)cc1)c1ccc(F)cc1</smiles>

The general procedure (Method C) was followed, and 20/1 hexane/ethyl acetate was used as the eluant to afford colorless oil (63.7 mg, 56\%), IR: 2956, 1721, 1501, 1156, $520 \mathrm{~cm}^{-1} .{ }^{1} \mathrm{H}$ NMR (500 MHz, Chloroform- $d$ ) $\delta 7.18-7.14(\mathrm{~m}, 2 \mathrm{H}), 7.07-7.03(\mathrm{~m}$, 2H), $6.89-6.83(\mathrm{~m}, 4 \mathrm{H}), 4.30(\mathrm{t}, \mathrm{J}=6.2 \mathrm{~Hz}, 2 \mathrm{H}), 3.20(\mathrm{t}, J=6.8 \mathrm{~Hz}, 2 \mathrm{H}), 1.97-1.91$ $(\mathrm{m}, 2 \mathrm{H}), 1.89-1.82(\mathrm{~m}, 2 \mathrm{H}) .{ }^{13} \mathrm{C}$ NMR $(125 \mathrm{MHz}$, Chloroform- $d) \delta 168.3,162.2(\mathrm{~J}=$ $247.8 \mathrm{~Hz}), 162.2(\mathrm{~J}=248.5 \mathrm{~Hz}), 142.9,137.9(\mathrm{~J}=3.7 \mathrm{~Hz}), 130.9(\mathrm{~J}=74.8 \mathrm{~Hz}), 130.9$ $(J=74.8 \mathrm{~Hz}), 131.0(J=3.5 \mathrm{~Hz}), 115.4(J=32.5 \mathrm{~Hz}), 115.3(J=32.5 \mathrm{~Hz}), 99.3,64.7$, 29.9, 29.3, 5.4. ${ }^{19} \mathrm{~F}$ NMR (471 MHz, Chloroform- $d$ ) $\delta$-111.7, -112.2. HRMS: (ESI) calculated for $\mathrm{C}_{19} \mathrm{H}_{17} \mathrm{~F}_{2} \mathrm{I}_{2} \mathrm{O}_{2}[\mathrm{M}+\mathrm{H}]^{+}$568.9280, found 568.9275.

\section{4-iodobutyl (Z)-2,3-bis(4-chlorophenyl)-3-iodoacrylate (4c)}<smiles>O=C(OCCCCI)/C(=C(\I)c1ccc(Cl)cc1)c1ccc(Cl)cc1</smiles>

The general procedure (Method C) was followed, and 20/1 hexane/ethyl acetate was used as the eluant to afford colorless oil (60.2 mg, 50\%), IR: 2955, 1721, 1169, 746, $488 \mathrm{~cm}^{-1} .{ }^{1} \mathrm{H}$ NMR $(500 \mathrm{MHz}$, Chloroform- $d) \delta 7.18-7.09(\mathrm{~m}, 6 \mathrm{H}), 7.02-7.00(\mathrm{~m}$, $2 \mathrm{H}), 4.31-4.28(\mathrm{~m}, 2 \mathrm{H}), 3.19(\mathrm{t}, J=6.6 \mathrm{~Hz}, 2 \mathrm{H}), 1.93(\mathrm{~m}, 2 \mathrm{H}), 1.84(\mathrm{~m}, 2 \mathrm{H}) .{ }^{13} \mathrm{C}$ NMR $(125 \mathrm{MHz}$, Chloroform- $d$ ) $\delta 168.1,143.0,140.2,134.5,134.4,133.3,130.6$, 130.0, 128.7, 128.5, 99.5, 64.8, 29.9, 29.3, 5.3. HRMS: (ESI) calculated for $\mathrm{C}_{19} \mathrm{H}_{17} \mathrm{Cl}_{2} \mathrm{I}_{2} \mathrm{O}_{2}[\mathrm{M}+\mathrm{H}]^{+}$600.8689, found 600.8689 .

\section{4-iodobutyl (Z)-2,3-bis(4-bromophenyl)-3-iodoacrylate (5c)}<smiles>O=C(OCCCCI)/C(=C(\I)c1ccc(Br)cc1)c1ccc(Br)cc1</smiles>

The general procedure (Method C) was followed, and 20/1 hexane/ethyl acetate was used as the eluant to afford colorless oil (62.1mg, 45\%), IR: 2955, 1721, 1168, 736, 500 $\mathrm{cm}^{-1} .{ }^{1} \mathrm{H}$ NMR (500 MHz, Chloroform- $d$ ) $\delta 7.33-7.30(\mathrm{~m}, 4 \mathrm{H}), 7.06-7.04(\mathrm{~m}, 2 \mathrm{H})$, $6.96-6.93(\mathrm{~m}, 2 \mathrm{H}), 4.29(\mathrm{t}, J=6.2 \mathrm{~Hz}, 2 \mathrm{H}), 3.19(\mathrm{t}, J=6.8 \mathrm{~Hz}, 2 \mathrm{H}), 1.97-1.91(\mathrm{~m}$, 2H), $1.88-1.80(\mathrm{~m}, 2 \mathrm{H}) .{ }^{13} \mathrm{C}$ NMR (125 MHz, Chloroform- $d$ ) $\delta$ 168.0, 143.0, 140.7, 
133.8, 131.7, 131.5, 130.8, 130.3, 122.8, 122.6, 99.5, 64.8, 29.9, 29.3, 5.4. HRMS: (ESI) calculated for $\mathrm{C}_{19} \mathrm{H}_{17} \mathrm{Br}_{2} \mathrm{I}_{2} \mathrm{O}_{2}[\mathrm{M}+\mathrm{H}]^{+} 688.7679$, found 688.7674 .

\section{4-iodobutyl (Z)-2-ethyl-3-iodopent-2-enoate (6c)}<smiles>CCC(C(=O)OCCCCI)=C(C)I</smiles>

The general procedure (Method C) was followed, and 20/1 hexane/ethyl acetate was used as the eluant to afford colorless oil (35.8 mg, 41\%), IR: 2969, 1721, 1132, 809, $491 \mathrm{~cm}^{-1} .{ }^{1} \mathrm{H}$ NMR (500 MHz, Chloroform- $d$ ) $\delta 4.22(\mathrm{t}, J=6.3 \mathrm{~Hz}, 2 \mathrm{H}), 3.24(\mathrm{t}, J=6.8$ $\mathrm{Hz}, 2 \mathrm{H}), 2.60(\mathrm{q}, J=7.4 \mathrm{~Hz}, 2 \mathrm{H}), 2.38(\mathrm{q}, J=7.5 \mathrm{~Hz}, 2 \mathrm{H}), 2.03-1.94(\mathrm{~m}, 2 \mathrm{H}), 1.88-$ $1.80(\mathrm{~m}, 2 \mathrm{H}), 1.10(\mathrm{t}, J=7.4 \mathrm{~Hz}, 3 \mathrm{H}), 1.06(\mathrm{t}, J=7.6 \mathrm{~Hz}, 3 \mathrm{H}) .{ }^{13} \mathrm{C} \mathrm{NMR}(125 \mathrm{MHz}$, Chloroform- $d$ ) $\delta$ 169.4, 141.8, 106.5, 63.8, 34.5, 30.0, 29.4, 24.7, 14.0, 13.2, 5.7. HRMS: (ESI) calculated for $\mathrm{C}_{11} \mathrm{H}_{19} \mathrm{I}_{2} \mathrm{O}_{2}[\mathrm{M}+\mathrm{H}]^{+} 436.9469$, found 436.9474 .

\section{4-bromobutyl (Z)-5-(4-ethylphenyl)-2,3-diphenylpent-2-en-4-ynoate (1d)}<smiles>CCc1ccc(C#CC(=C(C(=O)OCCCCBr)c2ccccc2)c2ccccc2)cc1</smiles>

The general procedure was followed $\left(12 \mathrm{~h}, 25^{\circ} \mathrm{C}\right)$, and 20/1 hexane/ethyl acetate was used as the eluant to afford colorless oil (67.3 mg, 69\%), IR: 2953, 1718, 1223, 1172, $697 \mathrm{~cm}^{-1} .{ }^{1} \mathrm{H}$ NMR (400 MHz, Chloroform- $d$ ) $\delta 7.40(\mathrm{~d}, J=8.1 \mathrm{~Hz}, 2 \mathrm{H}), 7.32-7.27$ $(\mathrm{m}, 2 \mathrm{H}), 7.21-7.16(\mathrm{~m}, 8 \mathrm{H}), 7.14-7.10(\mathrm{~m}, 2 \mathrm{H}), 4.32(\mathrm{t}, J=6.0 \mathrm{~Hz}, 2 \mathrm{H}), 3.28(\mathrm{t}, J=$ $6.2 \mathrm{~Hz}, 2 \mathrm{H}), 2.66(\mathrm{q}, J=7.7 \mathrm{~Hz}, 2 \mathrm{H}), 1.92-1.84(\mathrm{~m}, 4 \mathrm{H}), 1.23(\mathrm{~d}, J=7.6 \mathrm{~Hz}, 3 \mathrm{H}) .{ }^{13} \mathrm{C}$ NMR (125 MHz, Chloroform-d) $\delta$ 168.4, 145.4, 139.0, 137.3, 135.4, 131.7, 129.7, 129.6, 128.1, 128.0, 127.9, 127.9, 127.6, 120.0, 97.8, 88.7, 64.3, 33.0, 29.7, 29.3, 28.9, 27.3, 15.3. HRMS: (ESI) calculated for $\mathrm{C}_{29} \mathrm{H}_{28} \mathrm{BrO}_{2}[\mathrm{M}+\mathrm{H}]^{+} 487.1267$, found 487.1273.

\section{4-bromobutyl (Z)-2,3-diphenyl-3-(p-tolyl)acrylate (2d)}<smiles>Cc1ccc(C(=C(C(=O)OCCCCBr)c2ccccc2)c2ccccc2)cc1</smiles> 
The general procedure was followed $\left(12 \mathrm{~h}, 60^{\circ} \mathrm{C}\right)$, and 20/1 hexane/ethyl acetate was used as the eluant to afford colorless oil (54.9 mg, 61\%), IR: 2920, 1707, 1217, 731, $693 \mathrm{~cm}^{-1} .{ }^{1} \mathrm{H}$ NMR (400 MHz, Chloroform- $d$ ) $\delta 7.17-7.08(\mathrm{~m}, 12 \mathrm{H}), 7.01-6.98(\mathrm{~m}$, $2 \mathrm{H}), 4.01(\mathrm{t}, J=5.8 \mathrm{~Hz}, 2 \mathrm{H}), 3.26-3.18(\mathrm{~m}, 2 \mathrm{H}), 2.37(\mathrm{~s}, 3 \mathrm{H}), 1.57-1.52(\mathrm{~m}, 4 \mathrm{H})$. ${ }^{13} \mathrm{C}$ NMR (125 MHz, Chloroform- $d$ ) $\delta 170.7,146.2,140.7,139.6,138.0,137.7,133.0$, 130.9, 129.8, 129.1, 128.9, 128.1, 127.8, 127.6, 127.3, 63.9, 33.0, 29.0, 26.9, 21.3. HRMS: (ESI) calculated for $\mathrm{C}_{26} \mathrm{H}_{26} \mathrm{BrO}_{2}[\mathrm{M}+\mathrm{H}]^{+} 449.1111$, found 449.1112 .

but-3-en-1-yl (Z)-3-bromo-2,3-diphenylacrylate (3d)<smiles>C=CCCOC(=O)C(=C(Br)c1ccccc1)c1ccccc1</smiles>

The general procedure was followed $\left(4 \mathrm{~h}, 25^{\circ} \mathrm{C}\right)$, and 20/1 hexane/ethyl acetate was used as the eluant to afford colorless oil (45.1 mg, 63\%), IR: 2957, 1725, 1173, 692, $602 \mathrm{~cm}^{-1} .{ }^{1} \mathrm{H}$ NMR (400 MHz, Chloroform- $d$ ) $\delta 7.32-7.27(\mathrm{~m}, 2 \mathrm{H}), 7.25-7.17(\mathrm{~m}$, $6 \mathrm{H}), 7.16-7.12(\mathrm{~m}, 2 \mathrm{H}), 5.90$ - $5.79(\mathrm{~m}, 1 \mathrm{H}), 5.18-5.08(\mathrm{~m}, 2 \mathrm{H}), 4.40$ - $4.35(\mathrm{~m}, 2 \mathrm{H})$, $2.56-2.50(\mathrm{~m}, 2 \mathrm{H}) .{ }^{13} \mathrm{C}$ NMR (125 MHz, Chloroform- $d$ ) $\delta 167.8,138.3,137.1,134.9$, 133.6, 129.7, 128.9, 128.8, 128.3, 128.3, 128.1, 123.6, 117.4, 64.8, 32.8. HRMS: (ESI) calculated for $\mathrm{C}_{19} \mathrm{H}_{18} \mathrm{BrO}_{2}[\mathrm{M}+\mathrm{H}]^{+} 357.0485$, found 357.0491.

\section{4-(naphthalen-1-yloxy)butyl (Z)-3-bromo-2,3-diphenylacrylate (4d)}<smiles>O=C(OCCCCOc1cccc2ccccc12)/C(Br)=C(\Br)c1ccccc1</smiles>

The general procedure was followed $\left(24 \mathrm{~h}, 60{ }^{\circ} \mathrm{C}\right)$, and 20/1 hexane/ethyl acetate was used as the eluant to afford colorless oil (93.3 mg, 93\%), IR: 2956, 1723, 1267, 727, $692 \mathrm{~cm}^{-1} .{ }^{1} \mathrm{H}$ NMR (400 MHz, Chloroform- $d$ ) $\delta 8.28(\mathrm{~d}, J=7.4 \mathrm{~Hz}, 1 \mathrm{H}), 7.84-7.79$ (m, 1H), $7.55-7.28(\mathrm{~m}, 5 \mathrm{H}), 7.26-7.11(\mathrm{~m}, 9 \mathrm{H}), 6.80(\mathrm{dd}, J=7.6,2.4 \mathrm{~Hz}, 1 \mathrm{H}), 4.48$ $-4.40(\mathrm{~m}, 2 \mathrm{H}), 4.20-4.17(\mathrm{~m}, 2 \mathrm{H}), 2.08-2.06(\mathrm{~m}, 4 \mathrm{H}) .{ }^{13} \mathrm{C}$ NMR (125 MHz, Chloroform- $d$ ) $\delta 167.8,154.4,138.0,134.7,134.3,129.6,128.6,128.2,127.9,127.2$, 126.1, 125.6, 124.9, 123.4, 121.8, 120.0, 104.4, 67.1, 65.3, 25.8, 25.3. HRMS: (ESI) calculated for $\mathrm{C}_{29} \mathrm{H}_{26} \mathrm{BrO}_{3}[\mathrm{M}+\mathrm{H}]^{+}$501.1060, found 501.1061. 


\section{Reference}

1. Yao, L.; Hu, Q.; Bao, L.; Zhu, W.; Hu, Y., Fully Substituted Conjugate Benzofuran Core: Multiyne Cascade Coupling and Oxidation of Cyclopropenone. Org. Lett. 2021, 23, 4971-4975.

2. Nanda, T.; Biswal, P.; Pati, B. V.; Banjare, S. K.; Ravikumar, P. C., PalladiumCatalyzed C-C Bond Activation of Cyclopropenone: Modular Access to Trisubstituted $\alpha, \beta$-Unsaturated Esters and Amides. J. Org. Chem. 2021, 86, 2682-2695.

3. Chen, J.; Tang, B.; Liu, X.; Lv, G.; Shi, Y.; Huang, T.; Xing, H.; Guo, X.; Hai, L.; $\mathrm{Wu}, \mathrm{Y}$. , Ruthenium(ii)-catalyzed $[5+1]$ annulation reaction: a facile and efficient approach to construct 6-ethenyl phenanthridines utilizing a primary amine as a directing group. Org. Chem. Front. 2020, 7, 2944-2949.

4. JOSE, R. D. M. a. A., Reactions of Huisgen Zwitterions with Diphenyl Cyclopropenone: A Novel Strategy for the Synthesis of Oxazinone Derivatives. Asian J. Org. Chem. 2018, 30, 1075-1077.

5. Stach, T.; Dräger, J.; Huy, P. H., Nucleophilic Substitutions of Alcohols in High Levels of Catalytic Efficiency. Org. Lett. 2018, 20, 2980-2983.

6. Nguyen, S. S.; Ferreira, A. J.; Long, Z. G.; Heiss, T. K.; Dorn, R. S.; Row, R. D.; Prescher, J. A., Butenolide Synthesis from Functionalized Cyclopropenones. Org. Lett. 2019, 21, 8695-8699.

7. Row, R. D.; Prescher, J. A., A Cyclopropenethione-Phosphine Ligation for Rapid Biomolecule Labeling. Org. Lett. 2018, 20, 5614-5617.

8. Singh, S.; Ramamurthy, V., Regioselectivity in .alpha.-cleavage reactions: arylalkylcyclopropenethiones. J. Org. Chem. 1985, 50, 3732-3738.

9. Yang, Y.-L.; Zhang, Z.; Zhang, X.-N.; Wang, D.; Wei, Y.; Shi, M., Lewis basecatalyzed reactions of cyclopropenones: novel synthesis of mono- or multi-substituted allenic esters. Chem. Commun. 2014, 50, 115-117.

10. Heiss, T. K.; Prescher, J. A., Cyclopropeniminium Ions Exhibit Unique Reactivity Profiles with Bioorthogonal Phosphines. J. Org. Chem. 2019, 84, 7443-7448.

11. Hemming, K.; Khan, M. N.; Kondakal, V. V. R.; Pitard, A.; Qamar, M. I.; Rice, C. R., Pyridines from Azabicyclo[3.2.0]hept-2-en-4-ones through a Proposed Azacyclopentadienone. Org. Lett. 2012, 14, 126-129. 


\section{${ }^{1} \mathrm{H},{ }^{13} \mathrm{C}$ and ${ }^{19} \mathrm{~F}$ NMR Spectra of Products}

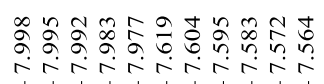
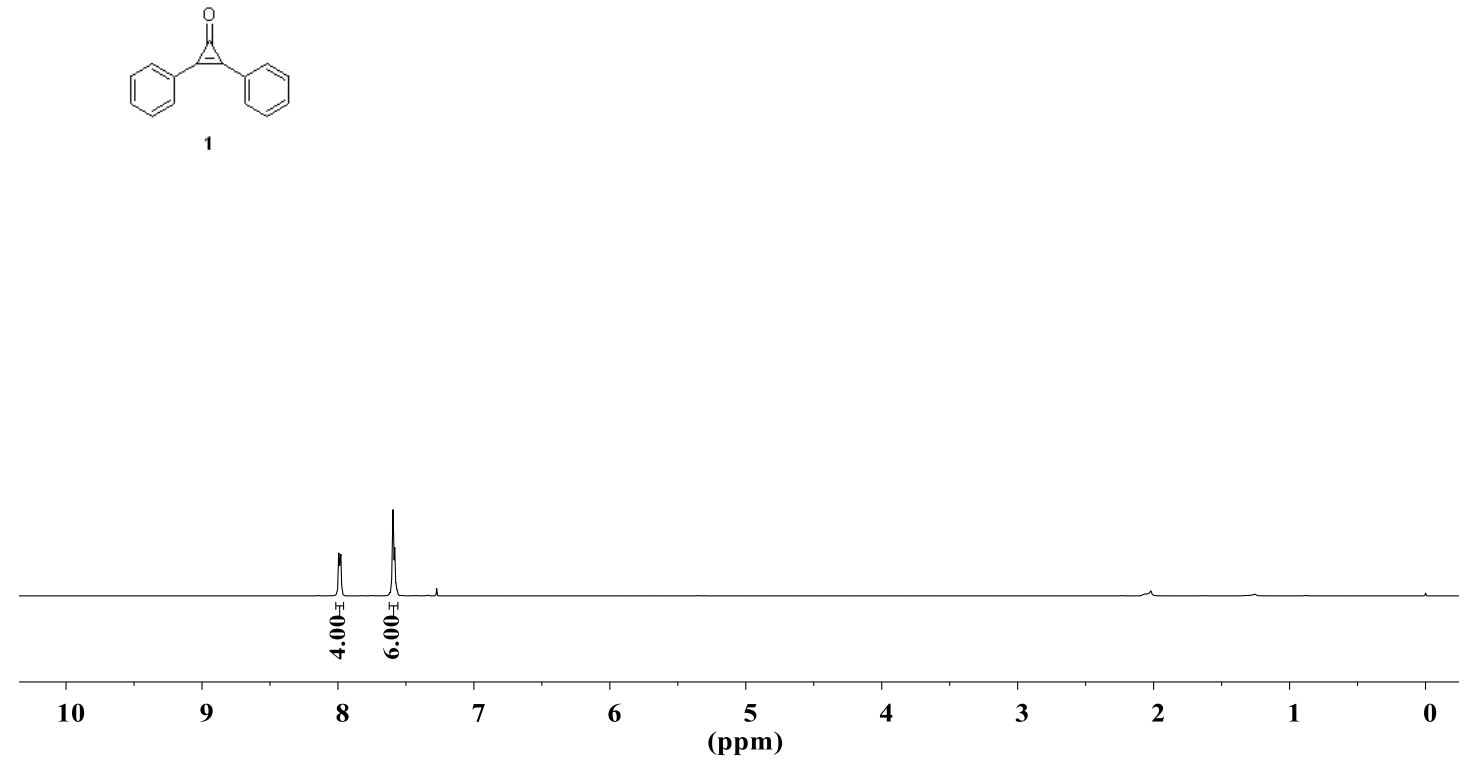

(500 $\mathrm{MHz}$ for ${ }^{1} \mathrm{H}$ NMR with $\mathrm{CDCl}_{3}$ as solvent)

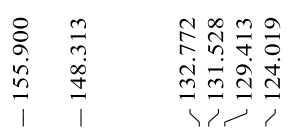

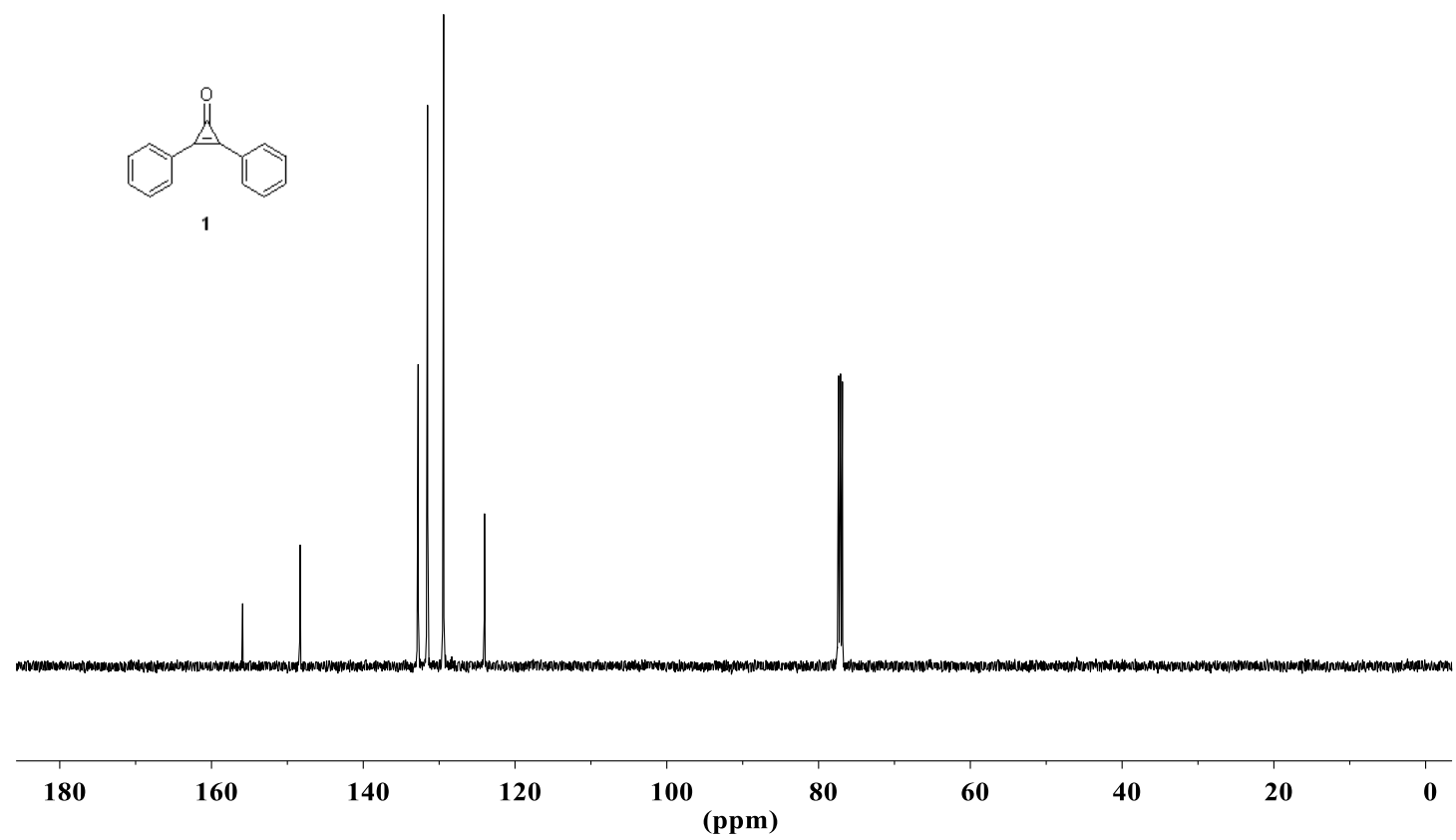

(125 MHz for ${ }^{13} \mathrm{C}$ NMR with $\mathrm{CDCl}_{3}$ as solvent) 


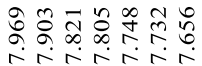

in
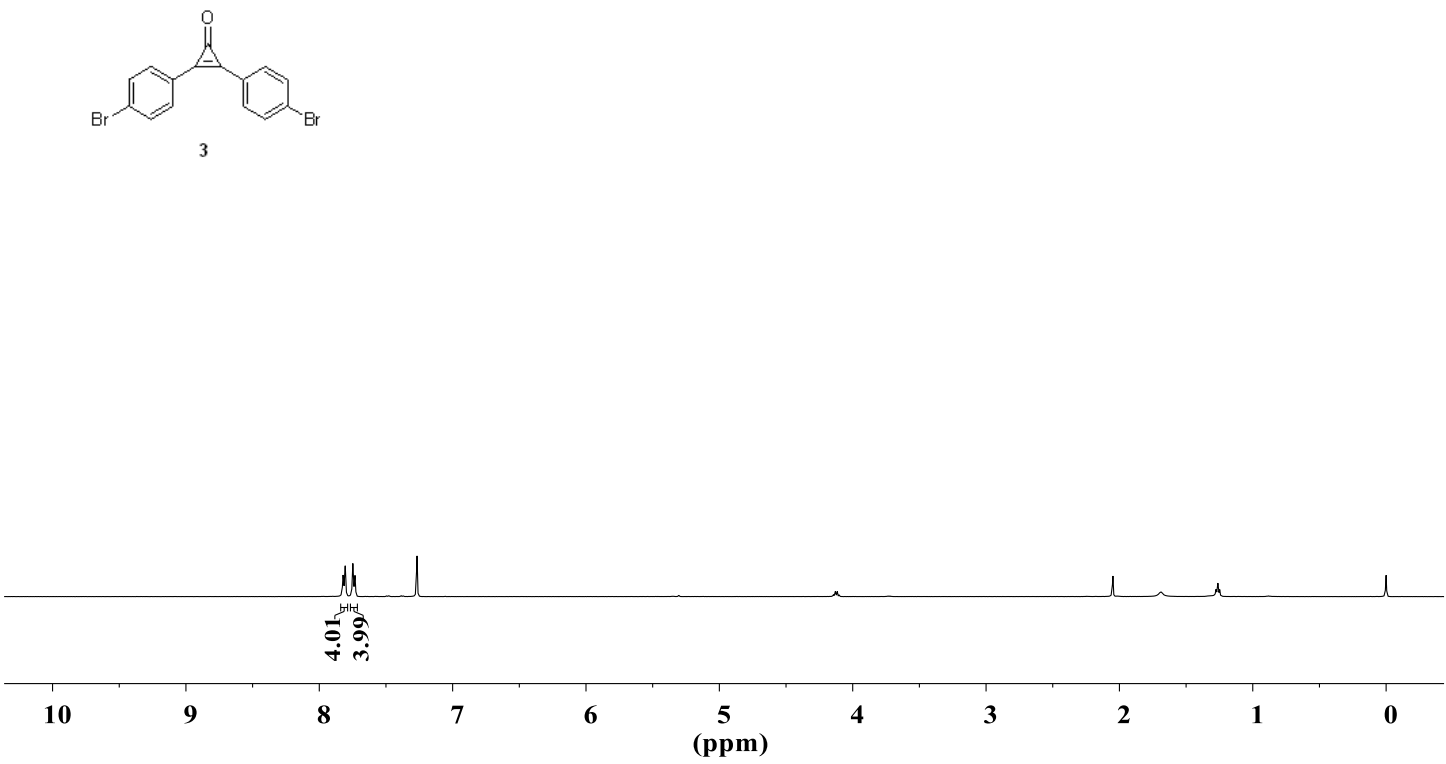

(500 MHz for ${ }^{1} \mathrm{H}$ NMR with $\mathrm{CDCl}_{3}$ as solvent)

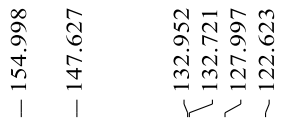
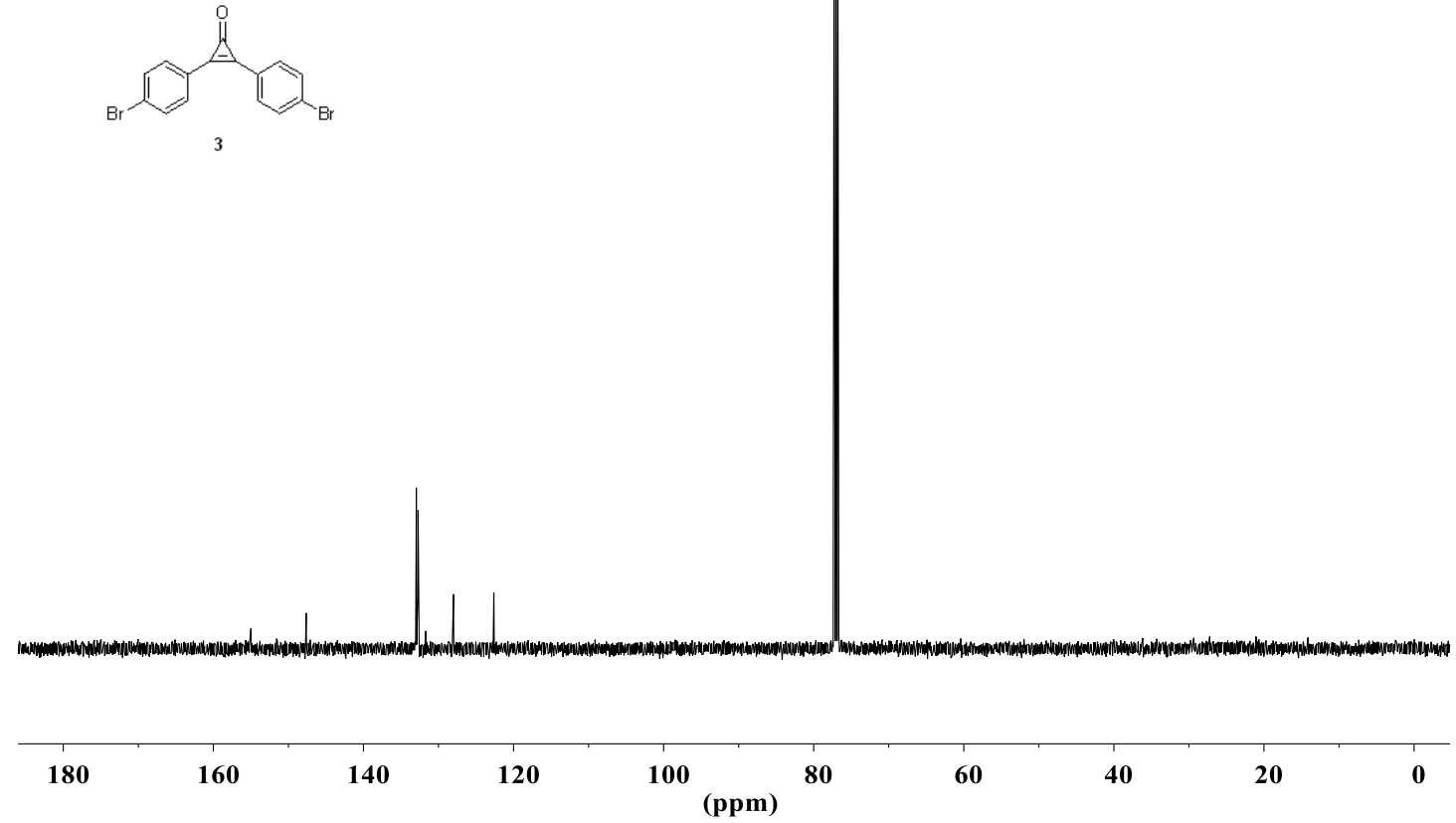

(125 MHz for ${ }^{13} \mathrm{C} \mathrm{NMR}$ with $\mathrm{CDCl}_{3}$ as solvent) 


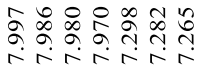

ritrir
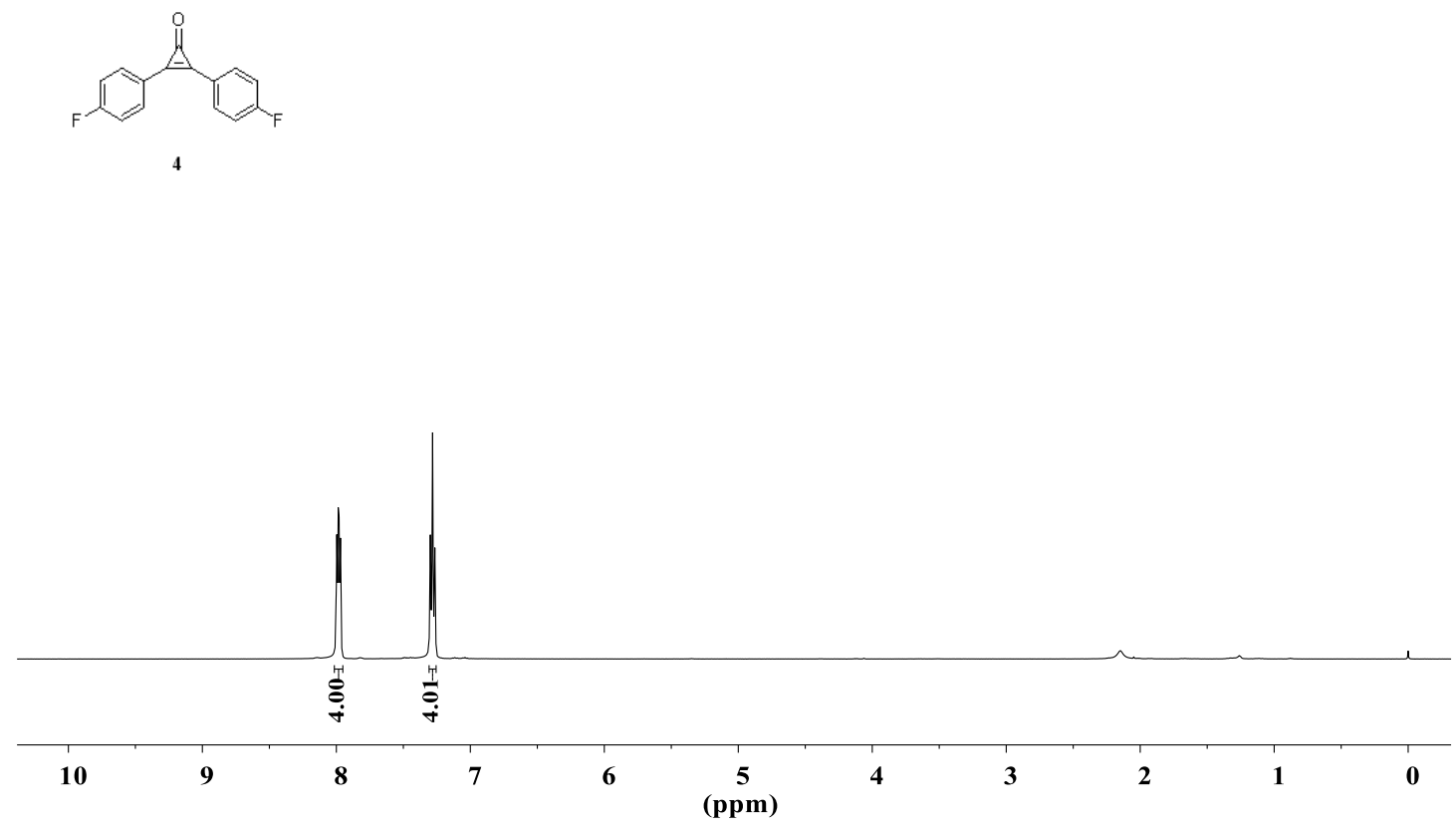

(500 $\mathrm{MHz}$ for ${ }^{1} \mathrm{H}$ NMR with $\mathrm{CDCl}_{3}$ as solvent)

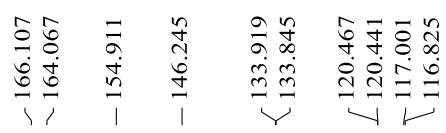

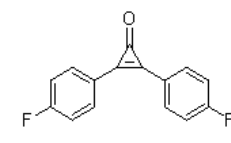

(125 MHz for ${ }^{13} \mathrm{C}$ NMR with $\mathrm{CDCl}_{3}$ as solvent) 


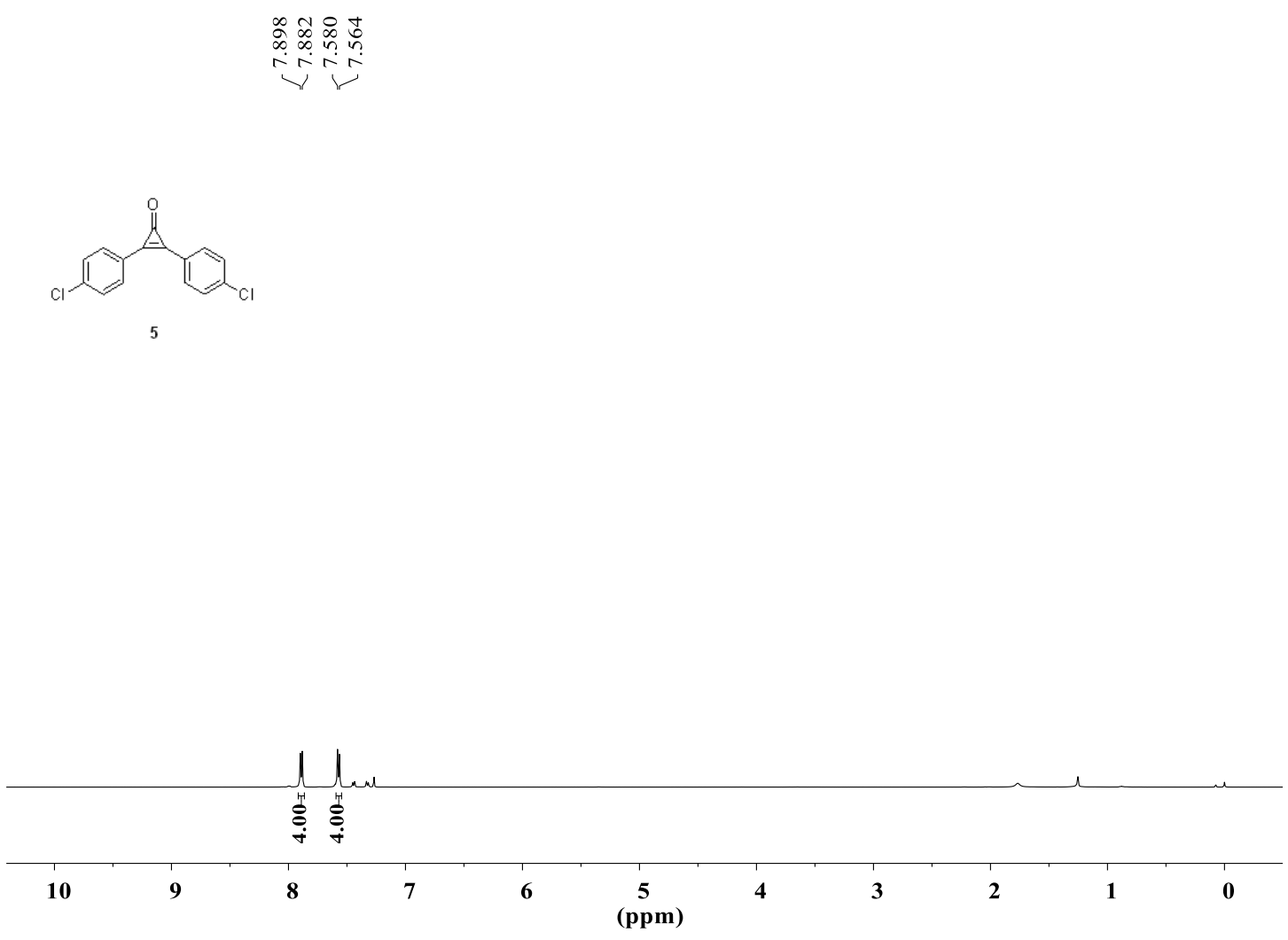

(500 $\mathrm{MHz}$ for ${ }^{1} \mathrm{H}$ NMR with $\mathrm{CDCl}_{3}$ as solvent)

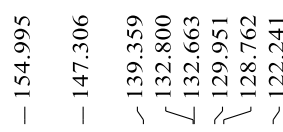
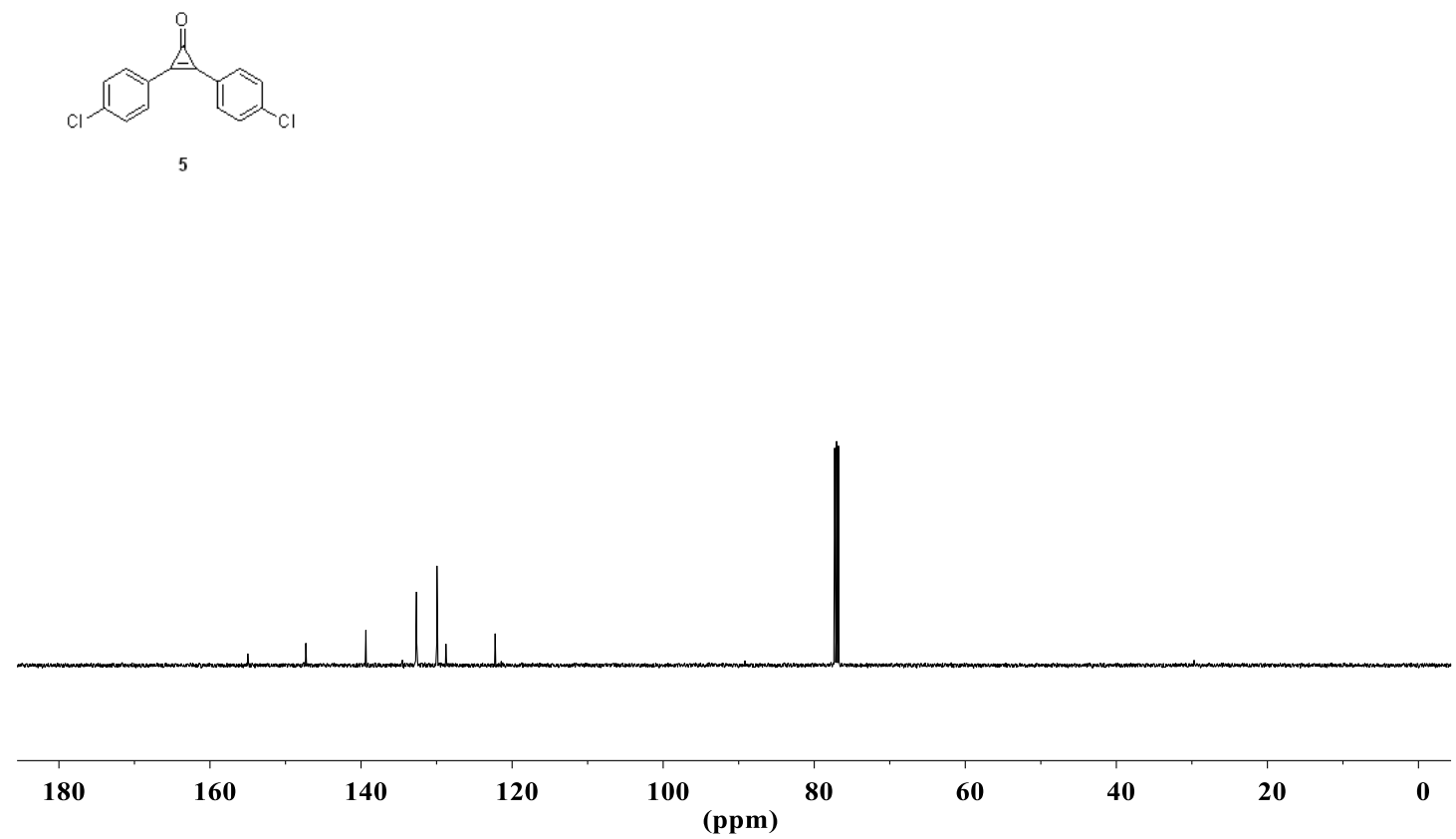

(125 MHz for ${ }^{13} \mathrm{C} \mathrm{NMR}$ with $\mathrm{CDCl}_{3}$ as solvent) 


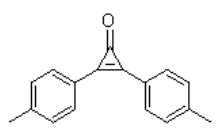

6

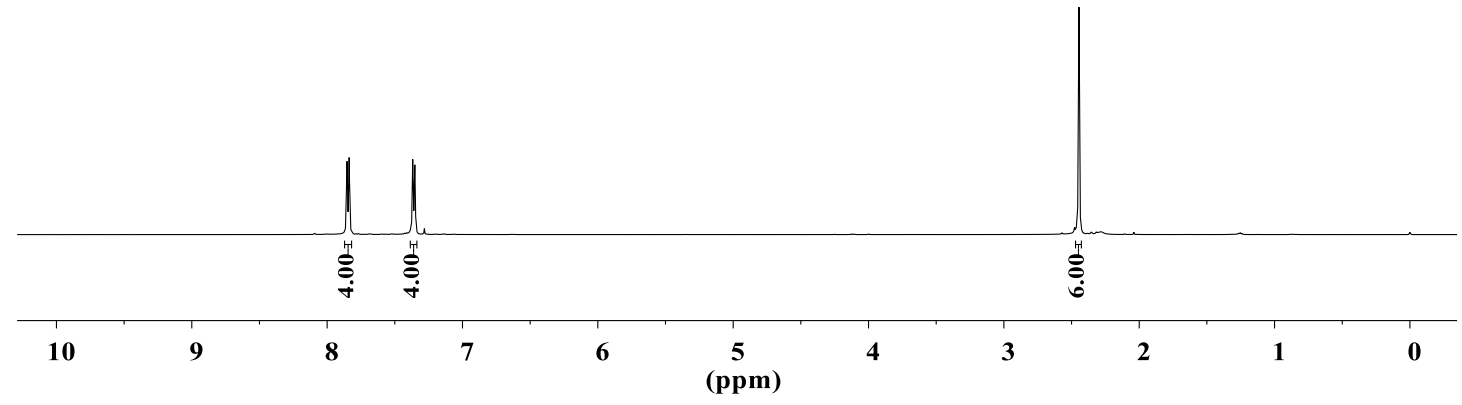

(500 MHz for ${ }^{1} \mathrm{H}$ NMR with $\mathrm{CDCl}_{3}$ as solvent)

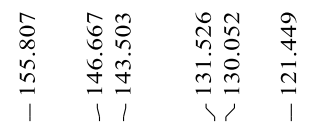

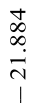

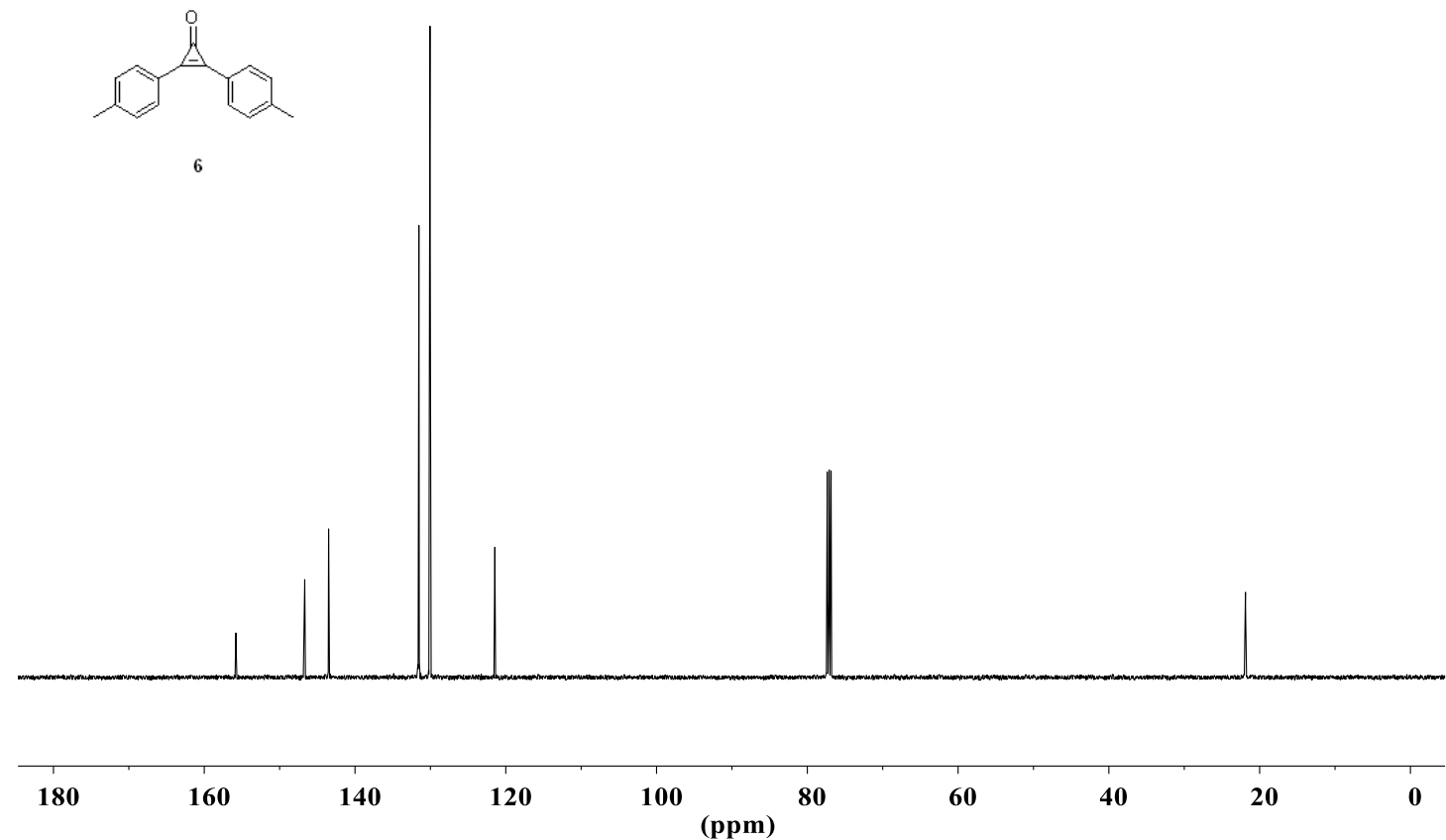

(125 $\mathrm{MHz}$ for ${ }^{13} \mathrm{C} \mathrm{NMR}$ with $\mathrm{CDCl}_{3}$ as solvent) 


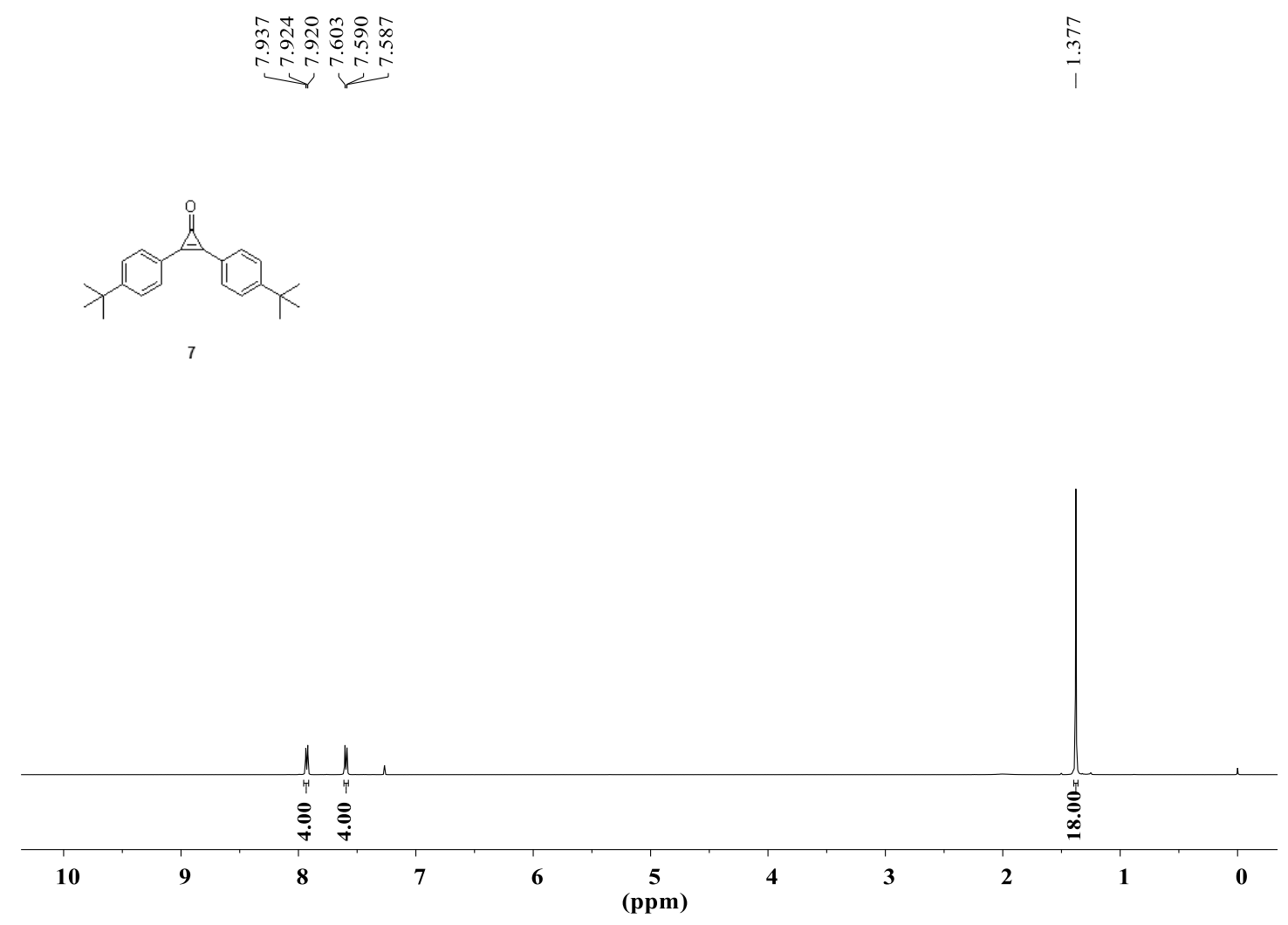

(500 MHz for ${ }^{1} \mathrm{H}$ NMR with $\mathrm{CDCl}_{3}$ as solvent)

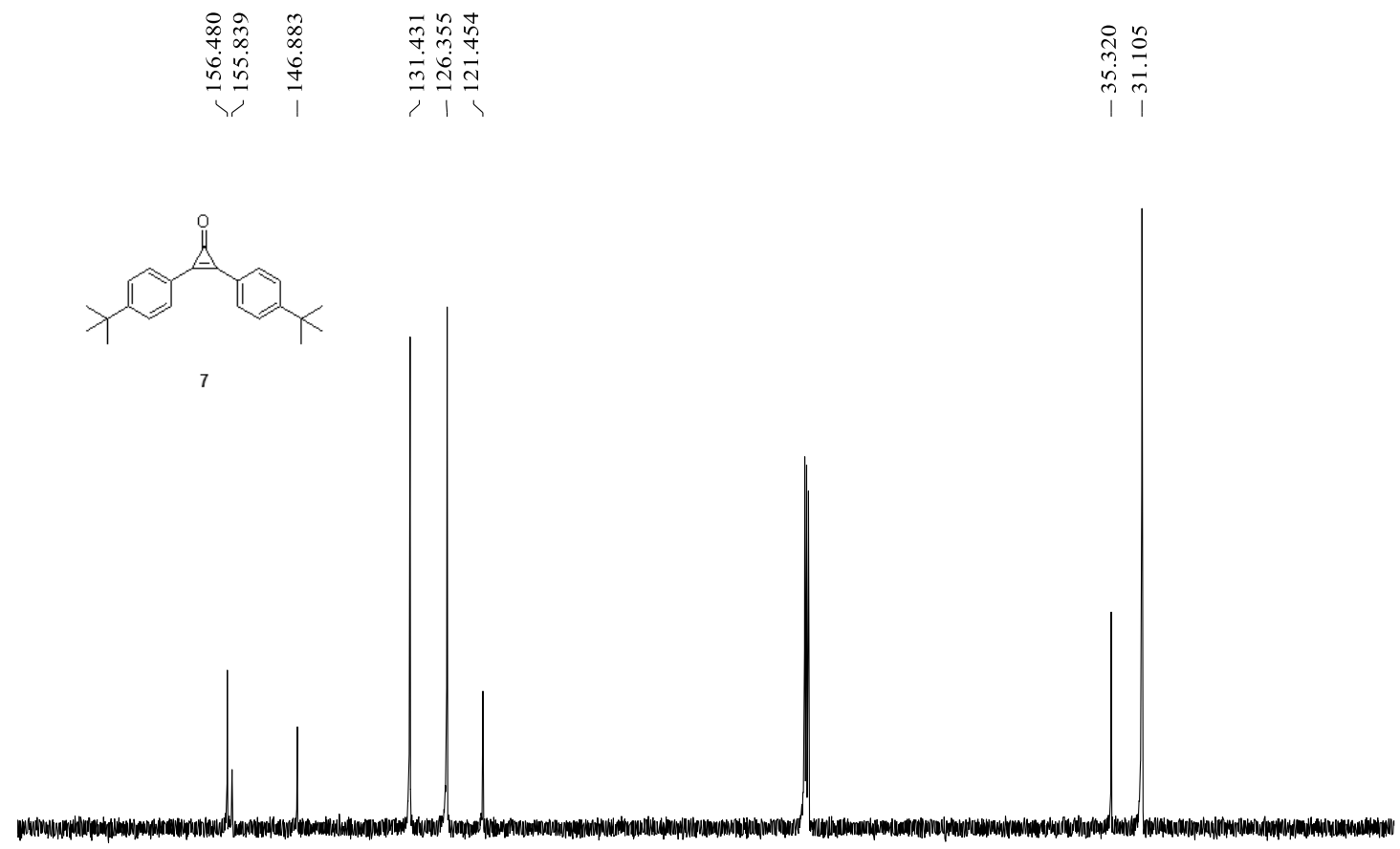

$\begin{array}{llllllllll}180 & 160 & 140 & 120 & 100 & 80 & 60 & 40 & 20 & 0\end{array}$

(125 MHz for ${ }^{13} \mathrm{C} \mathrm{NMR}$ with $\mathrm{CDCl}_{3}$ as solvent) 


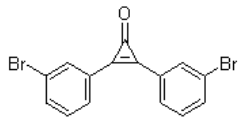

Mh

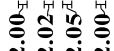

\begin{tabular}{llllllllllll}
\hline 10 & 9 & 8 & 7 & 6 & $\underset{(p p m)}{5}$ & 4 & 3 & 2 & 1 & 0
\end{tabular}

(500 MHz for ${ }^{1} \mathrm{H}$ NMR with $\mathrm{CDCl}_{3}$ as solvent)

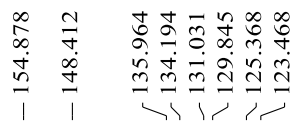

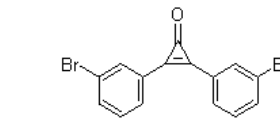

8

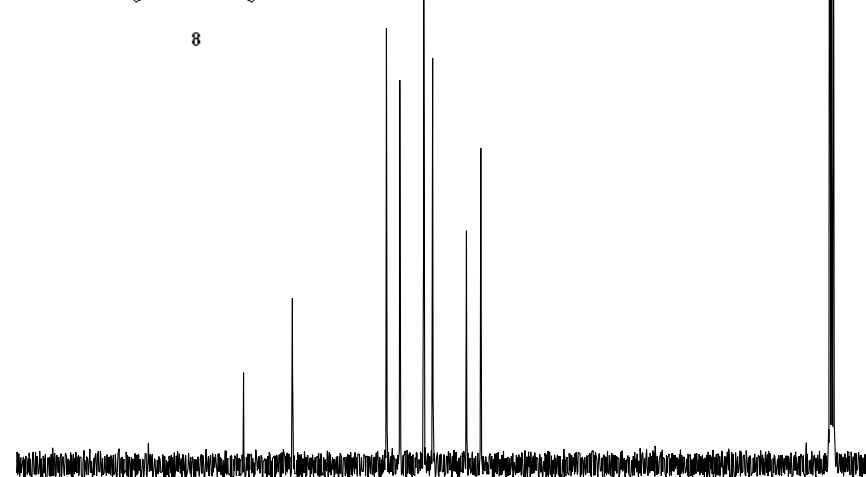

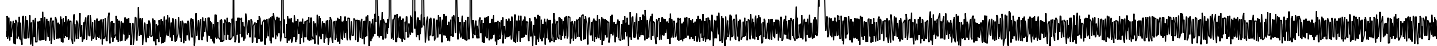

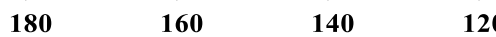

100

80

60

40

20

0

(125 $\mathrm{MHz}$ for ${ }^{13} \mathrm{C} \mathrm{NMR}$ with $\mathrm{CDCl}_{3}$ as solvent) 

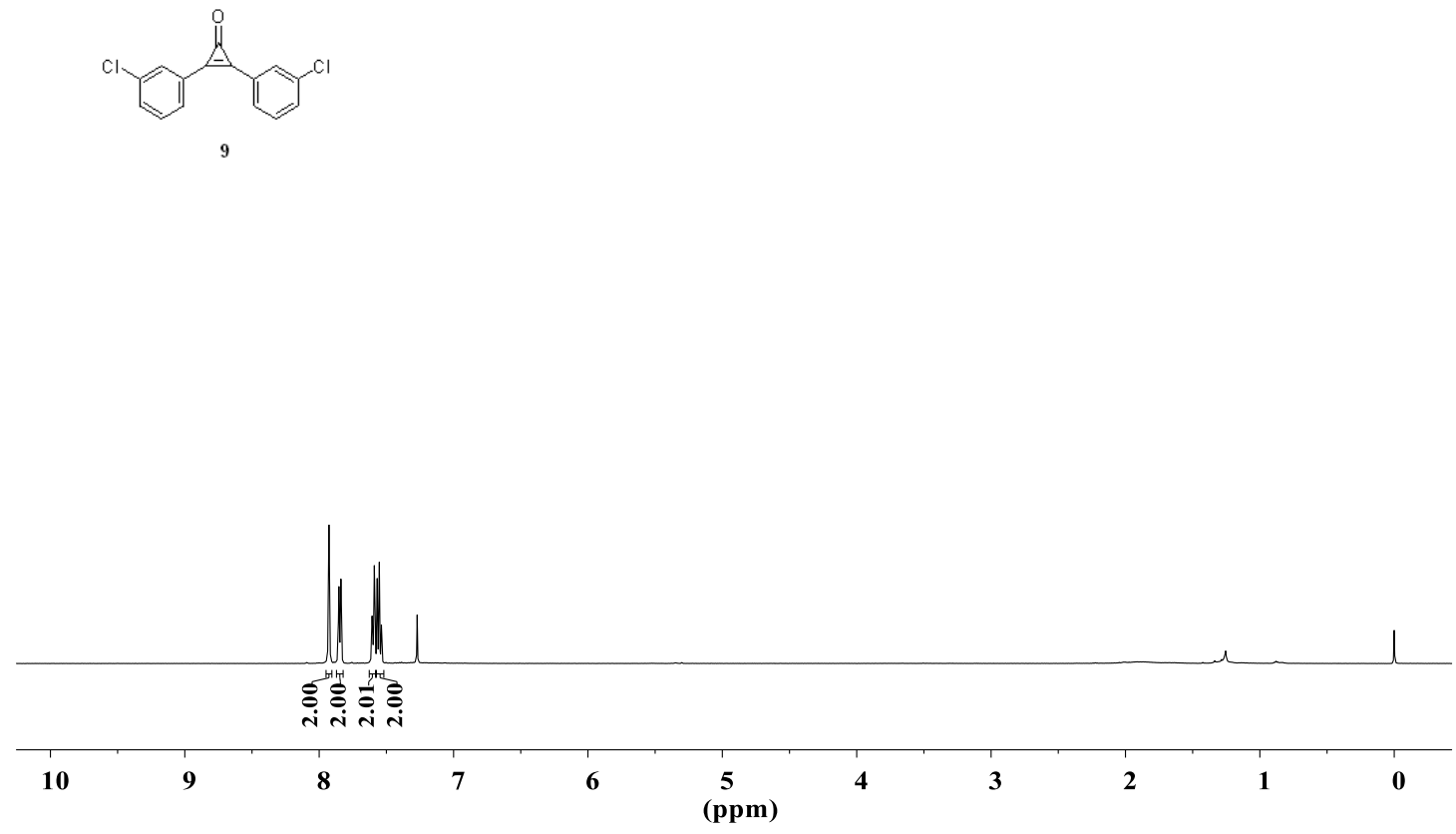

(500 MHz for ${ }^{1} \mathrm{H}$ NMR with $\mathrm{CDCl}_{3}$ as solvent)

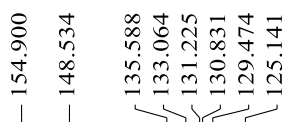
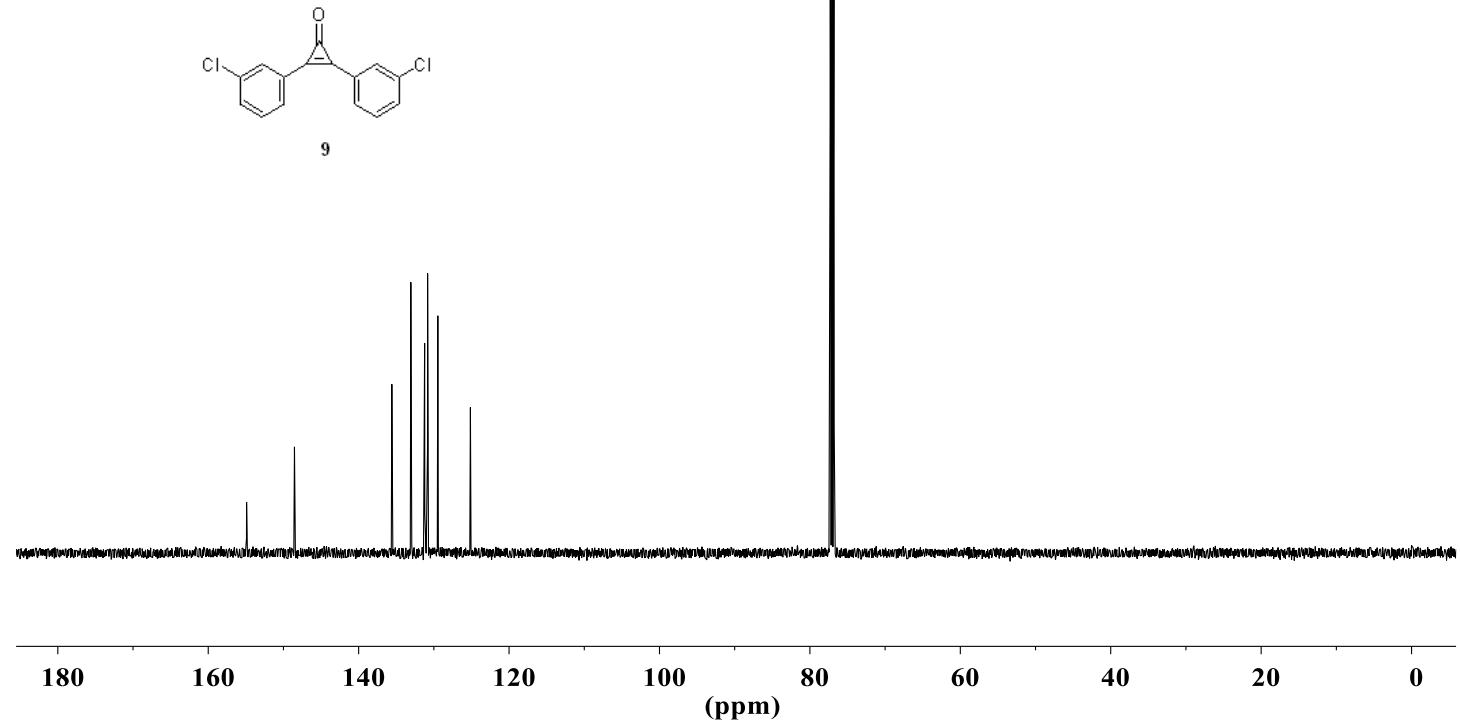

(125 $\mathrm{MHz}$ for ${ }^{13} \mathrm{C}$ NMR with $\mathrm{CDCl}_{3}$ as solvent) 


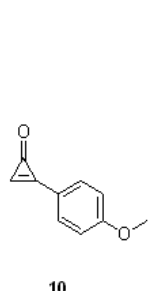

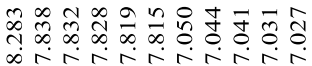

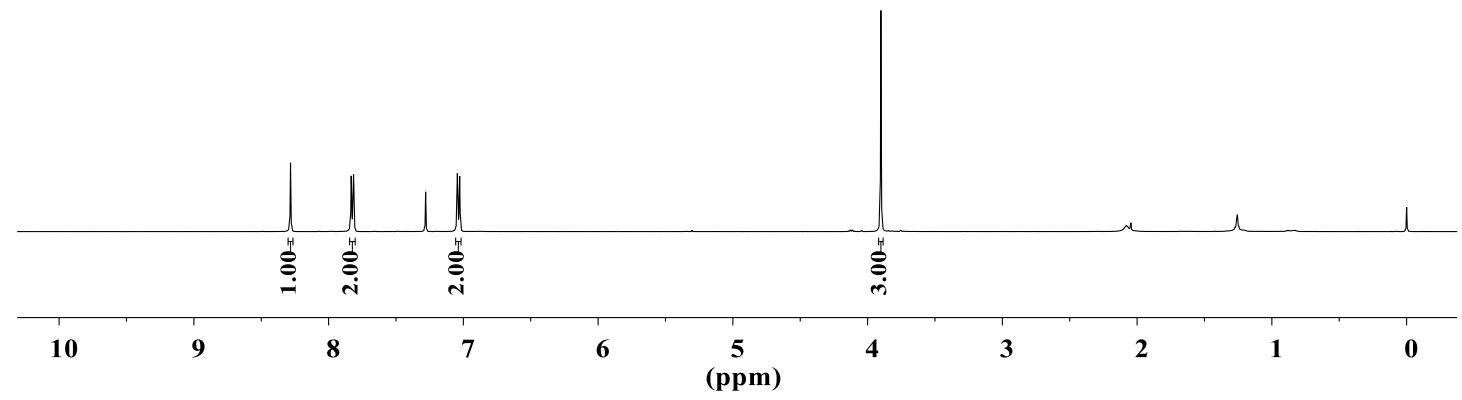

(500 MHz for ${ }^{1} \mathrm{H}$ NMR with $\mathrm{CDCl}_{3}$ as solvent)
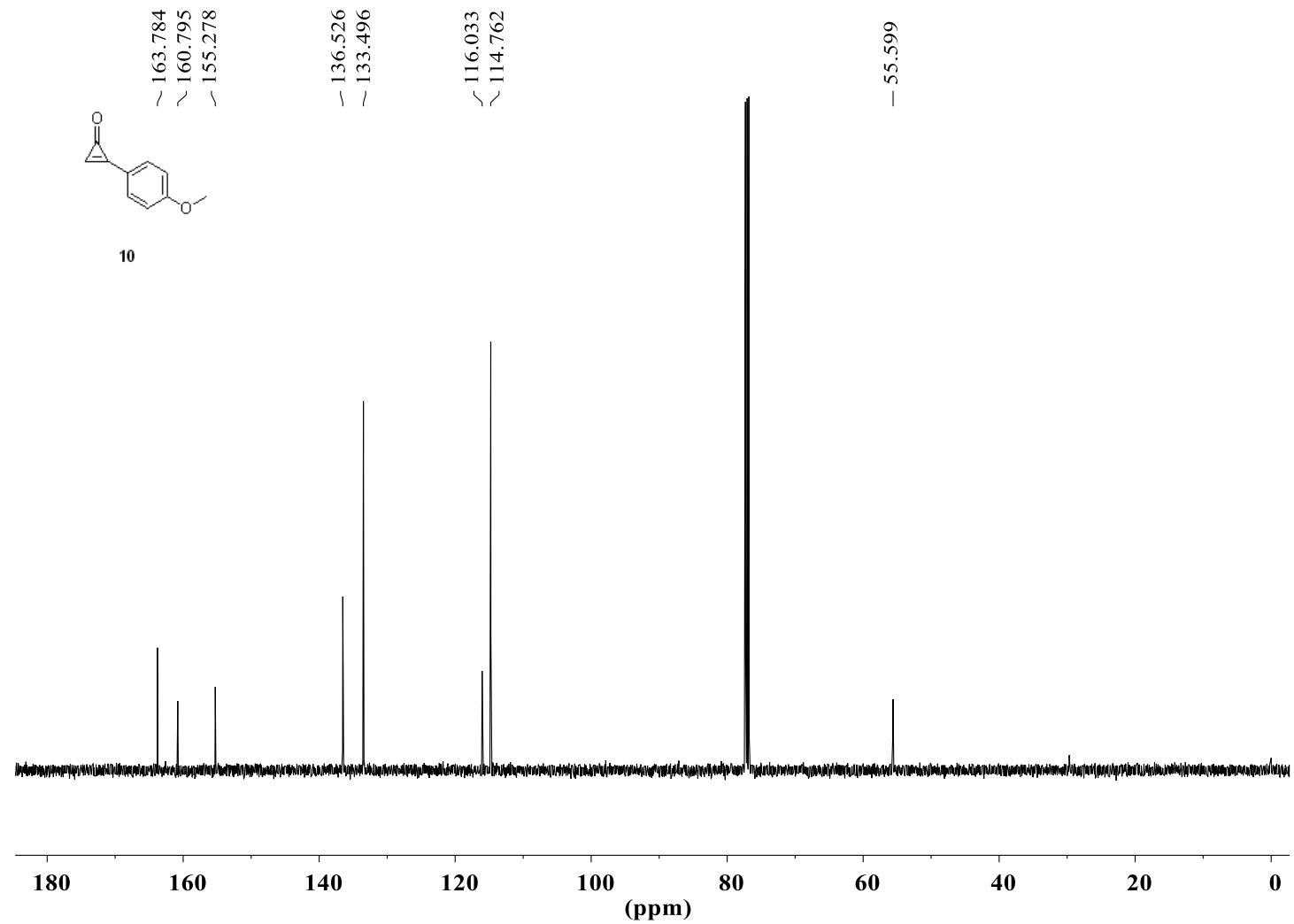

(125 $\mathrm{MHz}$ for ${ }^{13} \mathrm{C}$ NMR with $\mathrm{CDCl}_{3}$ as solvent) 


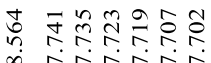

inara
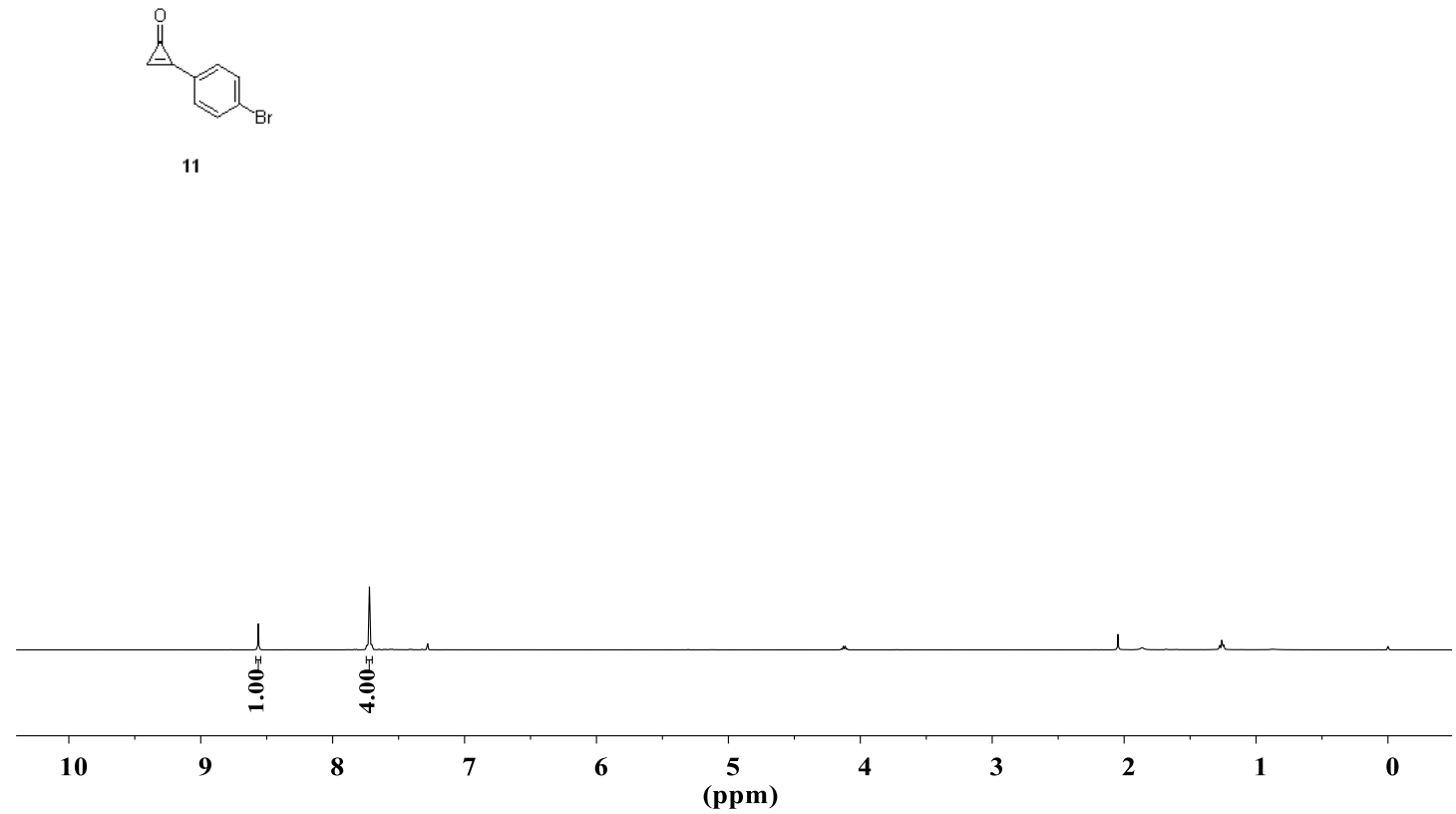

(500 MHz for ${ }^{1} \mathrm{H}$ NMR with $\mathrm{CDCl}_{3}$ as solvent)

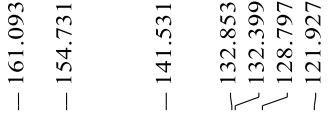
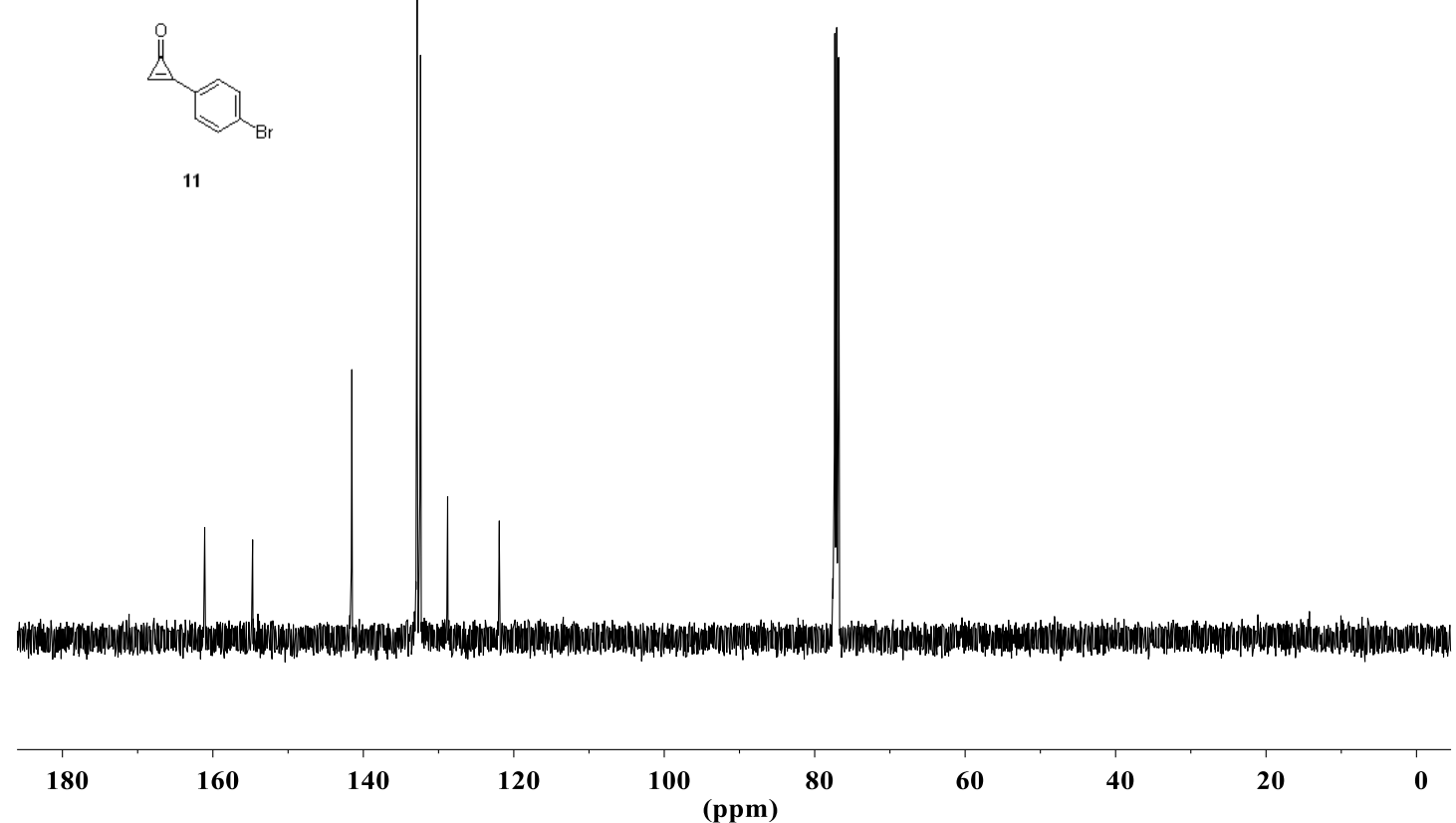

(125 $\mathrm{MHz}$ for ${ }^{13} \mathrm{C}$ NMR with $\mathrm{CDCl}_{3}$ as solvent) 


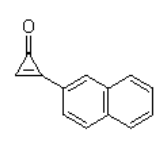

12

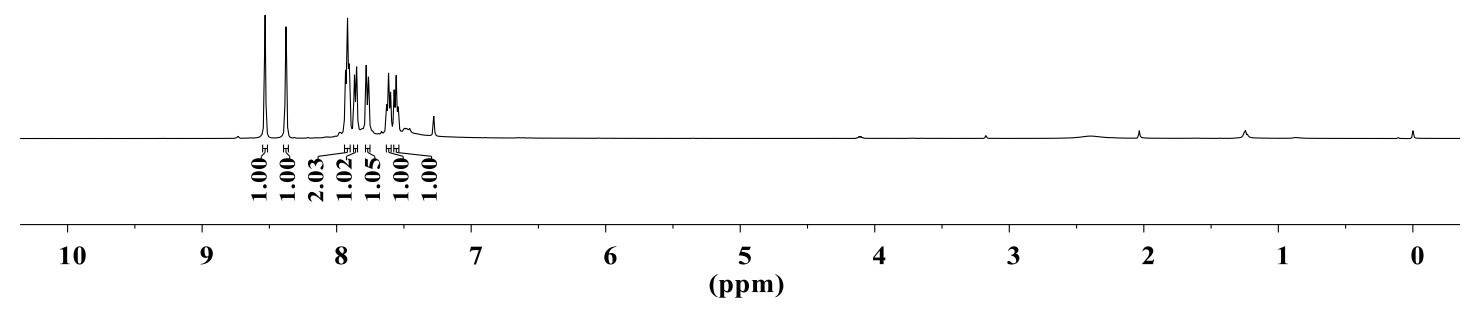

(500 $\mathrm{MHz}$ for ${ }^{1} \mathrm{H}$ NMR with $\mathrm{CDCl}_{3}$ as solvent)

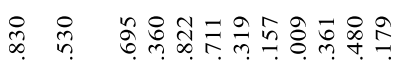

in ñ

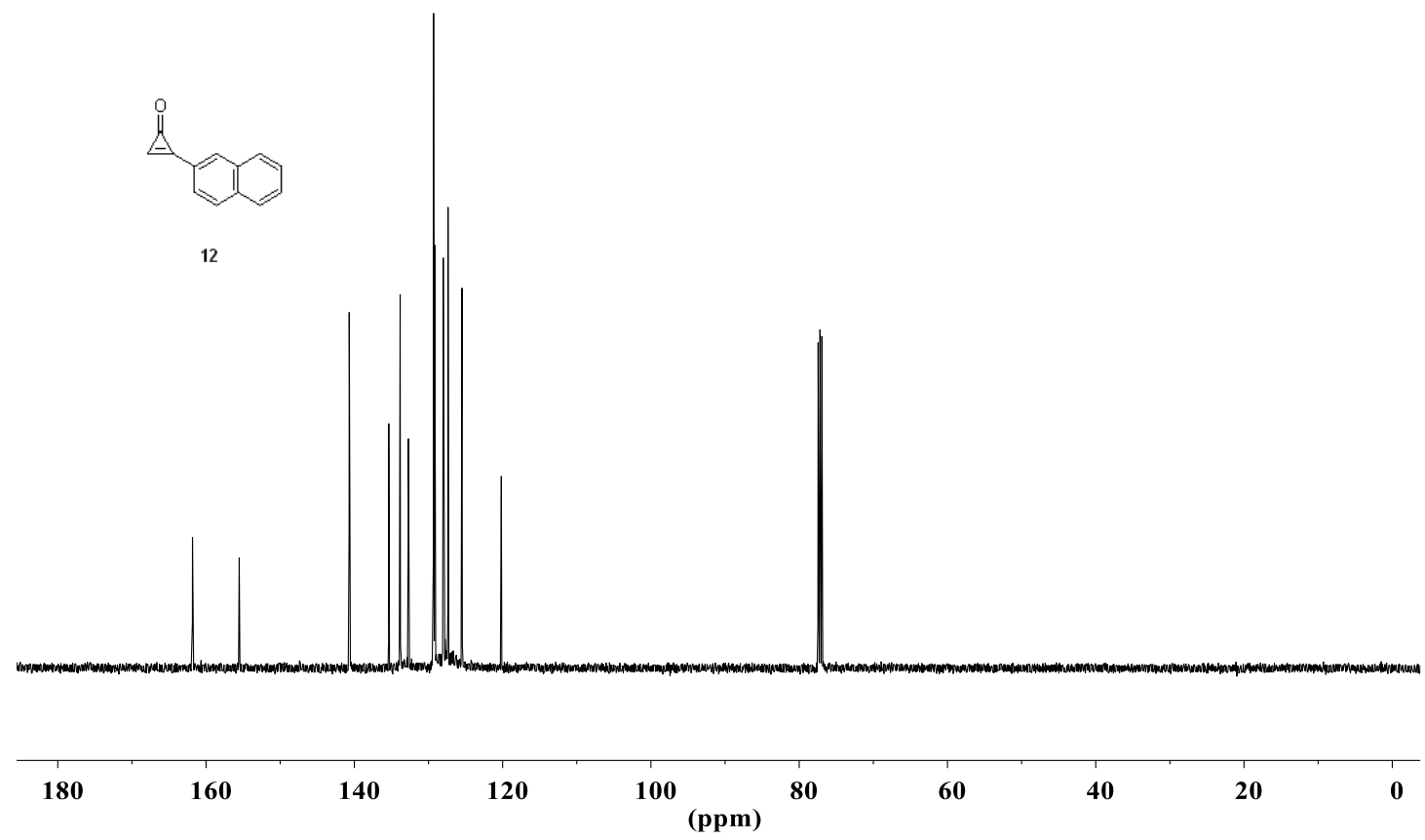

(125 $\mathrm{MHz}$ for ${ }^{13} \mathrm{C} \mathrm{NMR}$ with $\mathrm{CDCl}_{3}$ as solvent) 


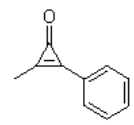

13

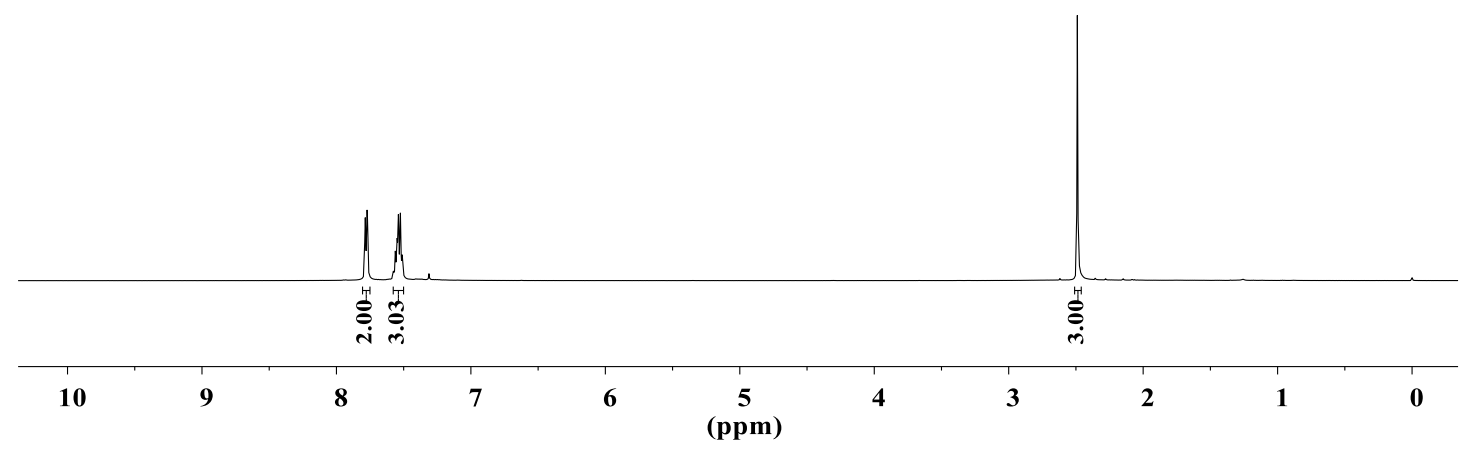

(500 MHz for ${ }^{1} \mathrm{H}$ NMR with $\mathrm{CDCl}_{3}$ as solvent)

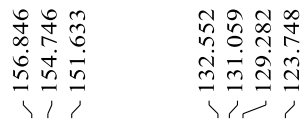

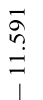

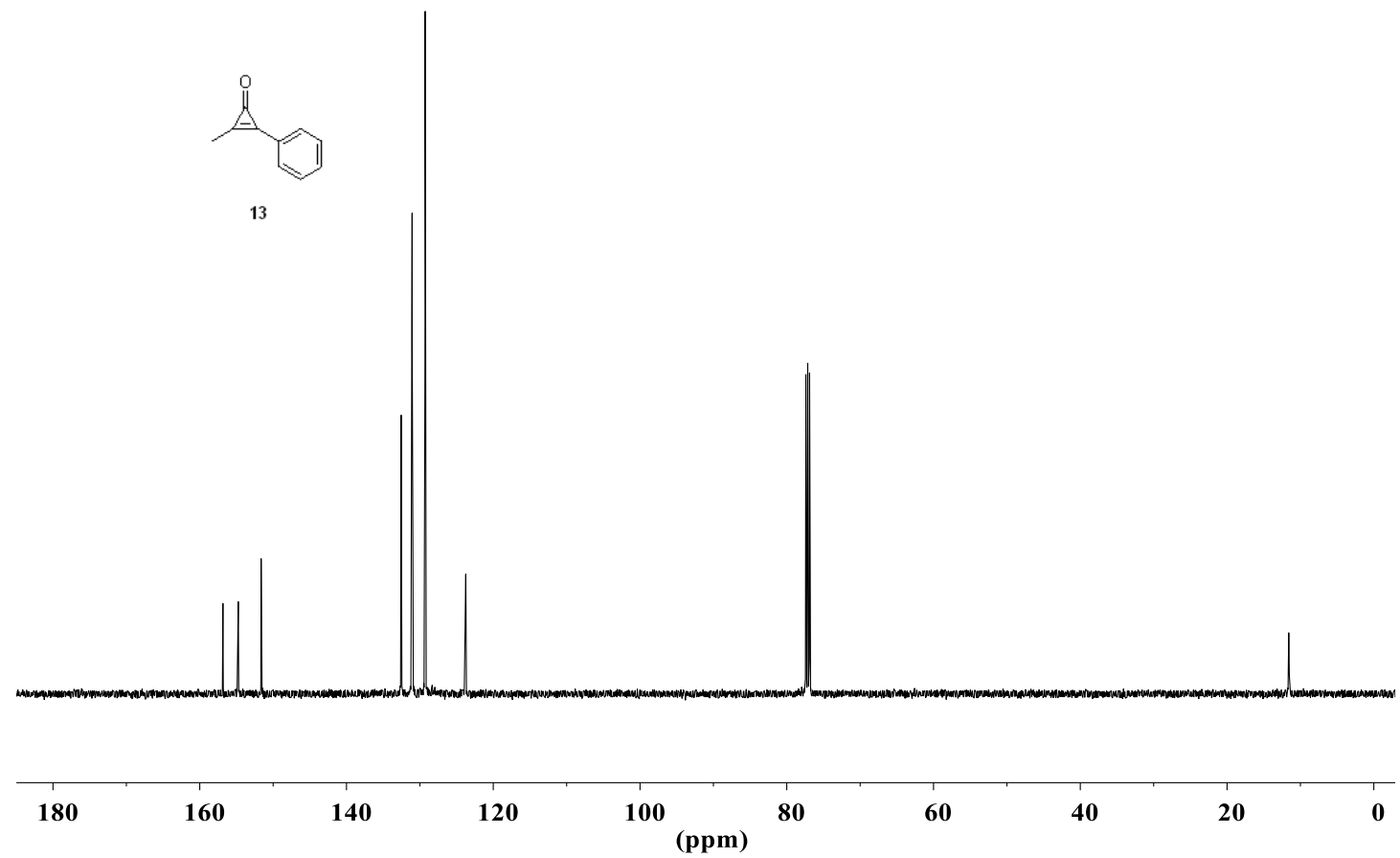

(125 MHz for ${ }^{13} \mathrm{C} \mathrm{NMR}$ with $\mathrm{CDCl}_{3}$ as solvent) 


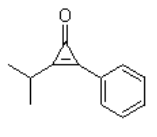

14

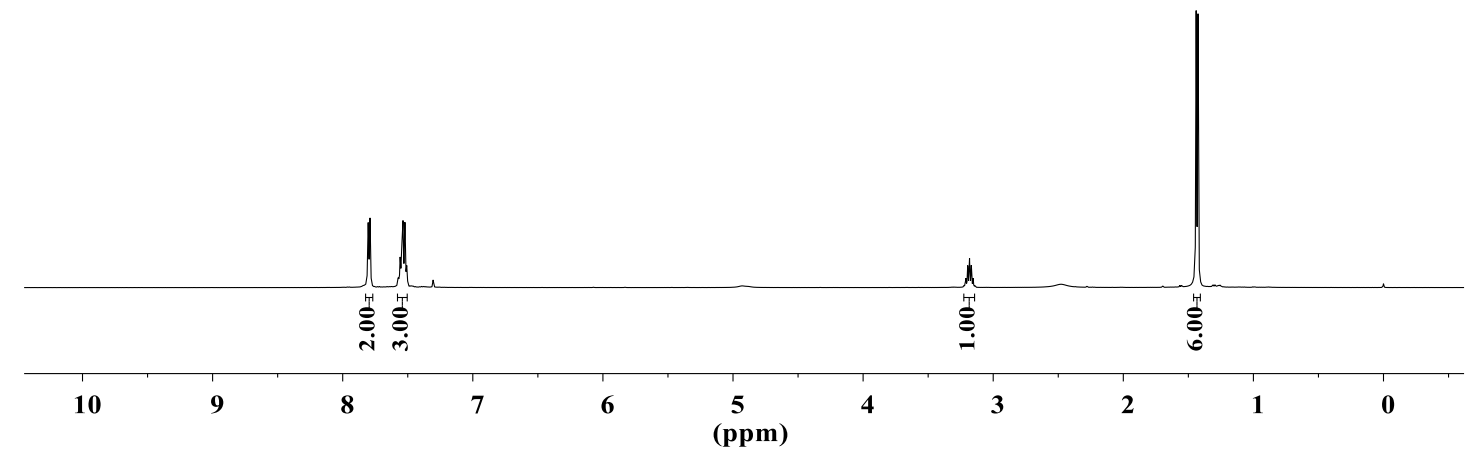

(500 MHz for ${ }^{1} \mathrm{H}$ NMR with $\mathrm{CDCl}_{3}$ as solvent)

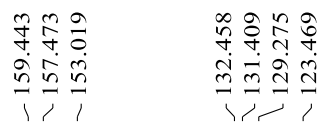

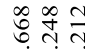

กิ่

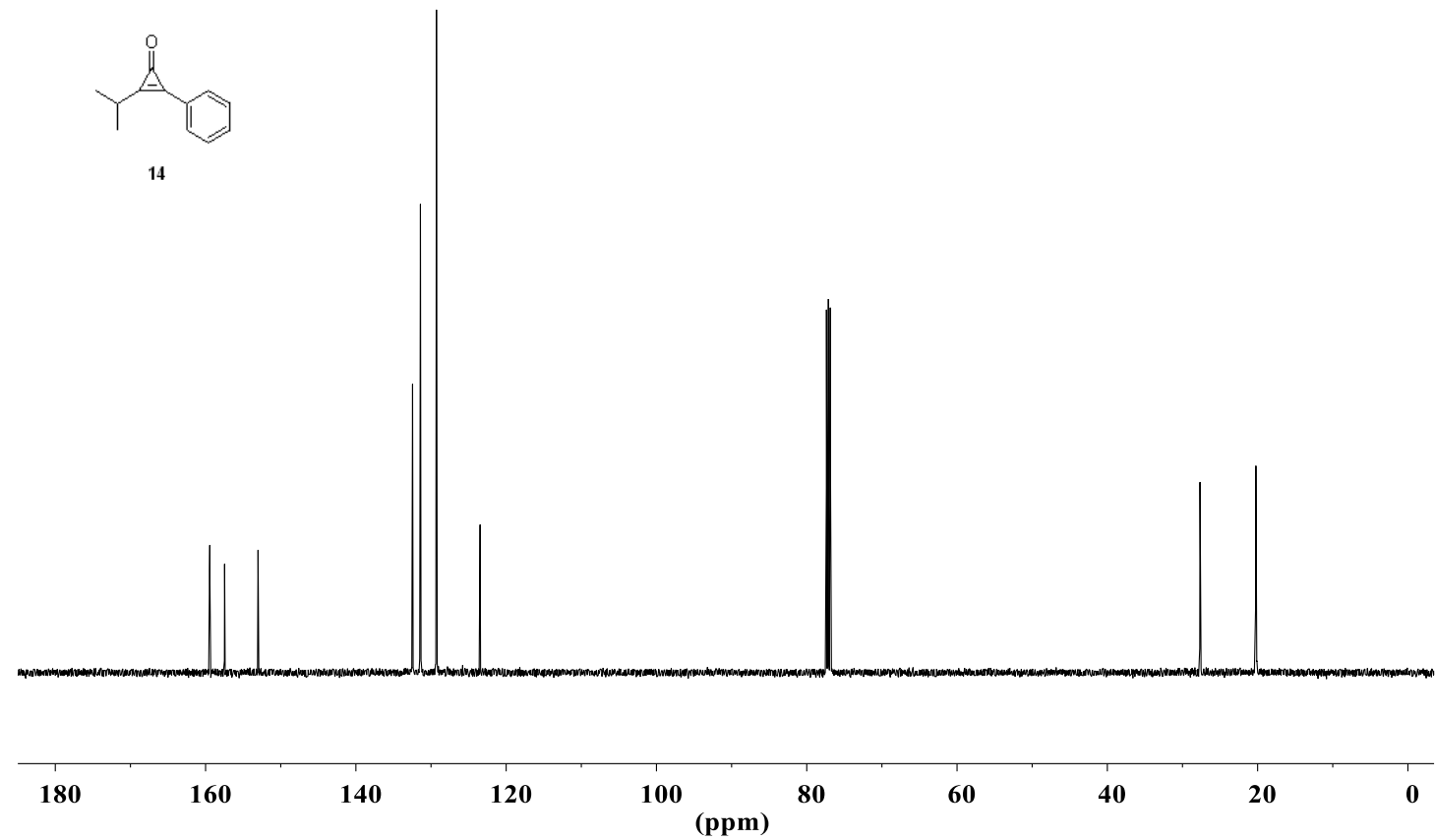

(125 $\mathrm{MHz}$ for ${ }^{13} \mathrm{C}$ NMR with $\mathrm{CDCl}_{3}$ as solvent) 


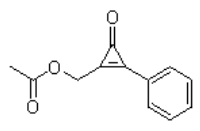

15

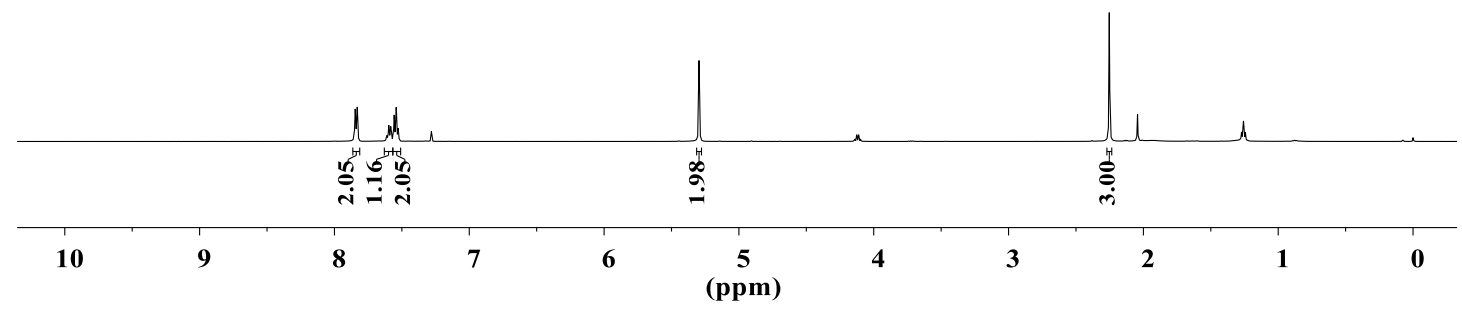

(500 MHz for ${ }^{1} \mathrm{H}$ NMR with $\mathrm{CDCl}_{3}$ as solvent)

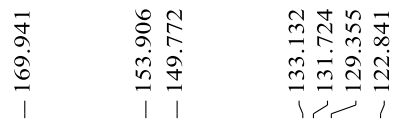

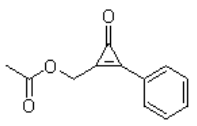

15

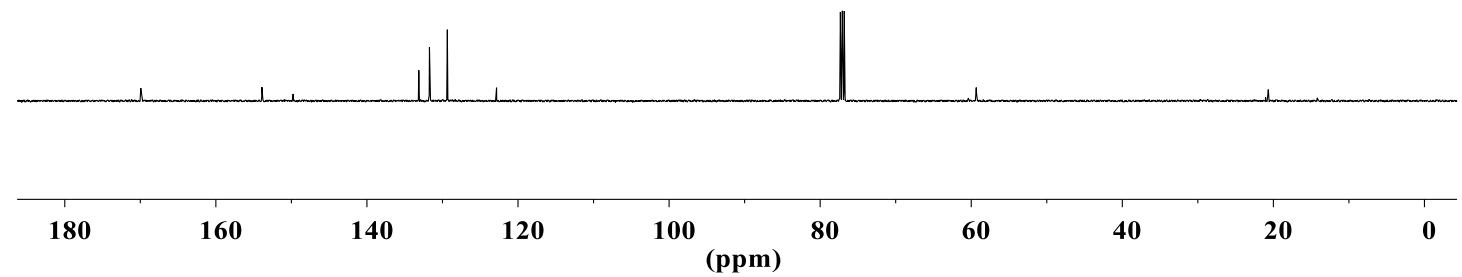

(125 MHz for ${ }^{13} \mathrm{C} \mathrm{NMR}$ with $\mathrm{CDCl}_{3}$ as solvent) 

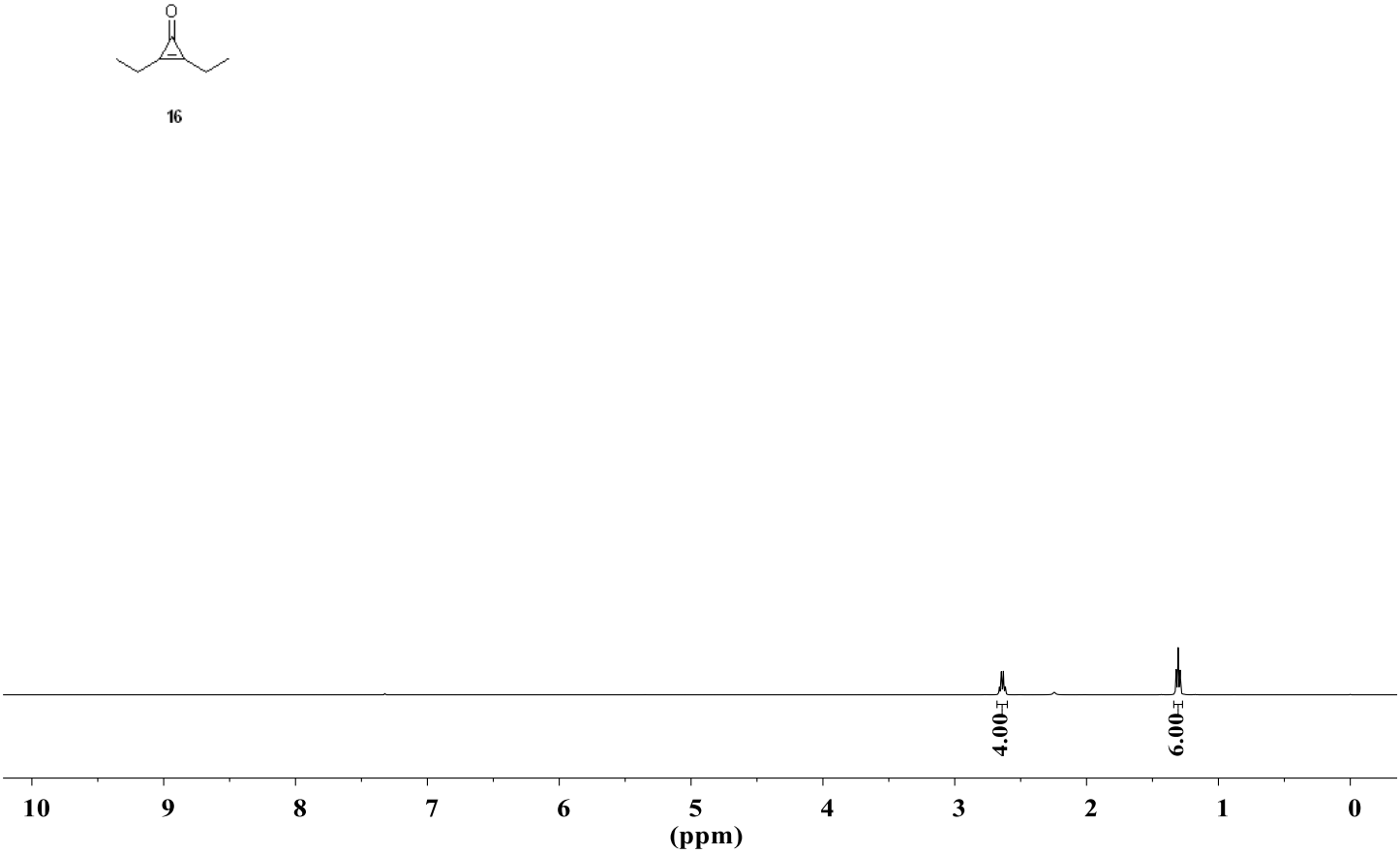

(500 MHz for ${ }^{1} \mathrm{H}$ NMR with $\mathrm{CDCl}_{3}$ as solvent)

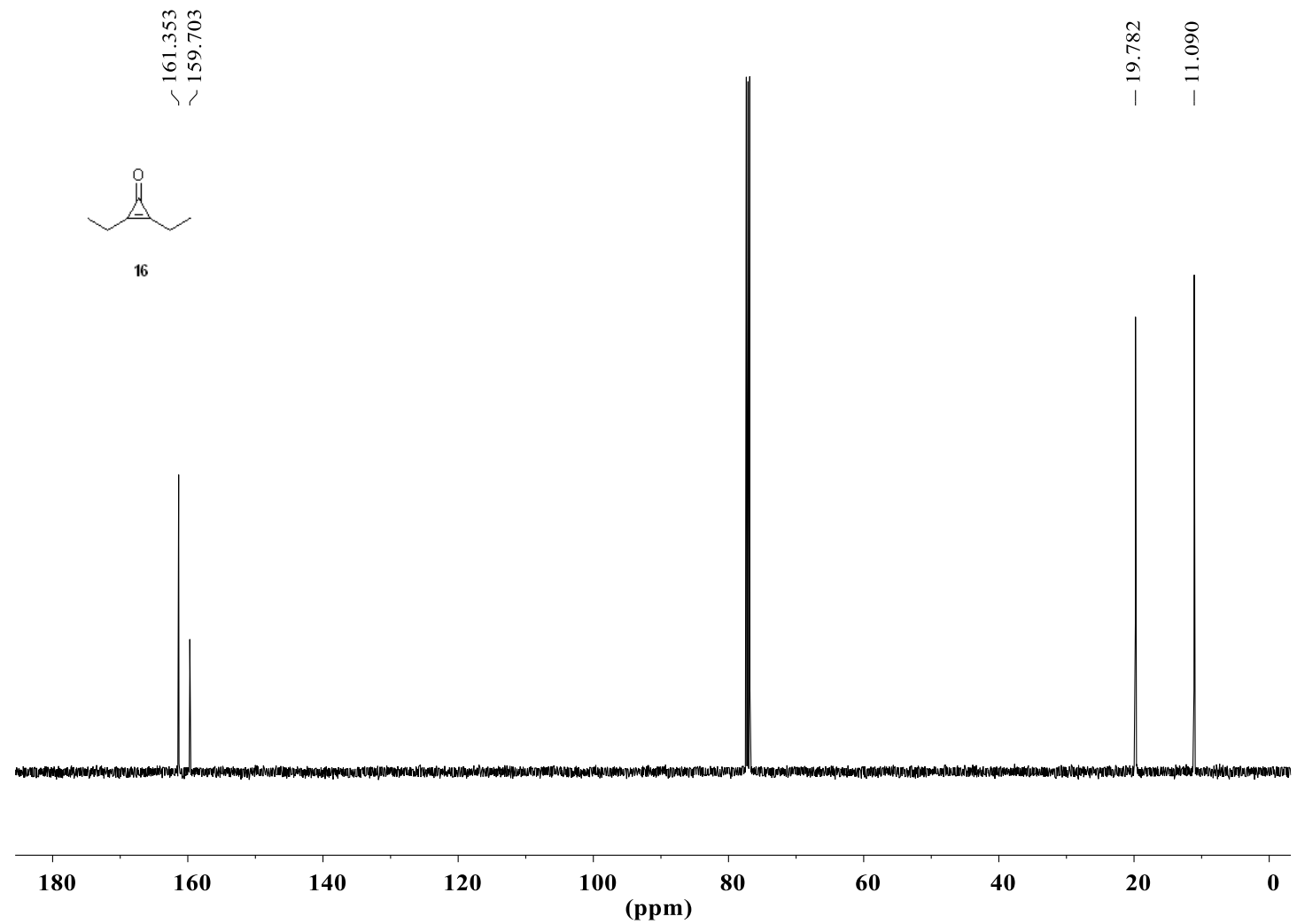

(125 MHz for ${ }^{13} \mathrm{C} \mathrm{NMR}$ with $\mathrm{CDCl}_{3}$ as solvent) 


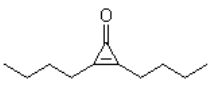

17

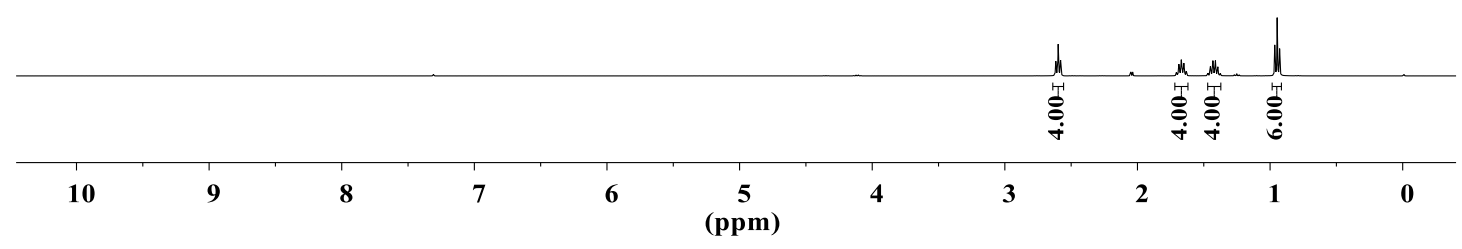

(500 MHz for ${ }^{1} \mathrm{H}$ NMR with $\mathrm{CDCl}_{3}$ as solvent)

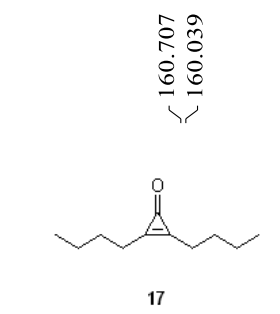

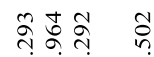

तथ

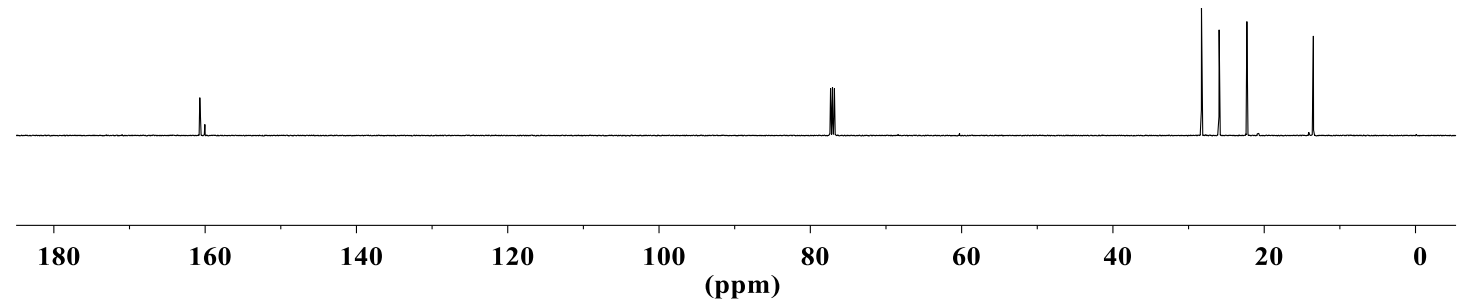

(125 $\mathrm{MHz}$ for ${ }^{13} \mathrm{C} \mathrm{NMR}$ with $\mathrm{CDCl}_{3}$ as solvent) 


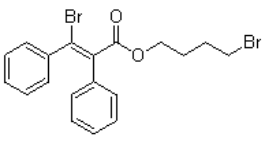

1a

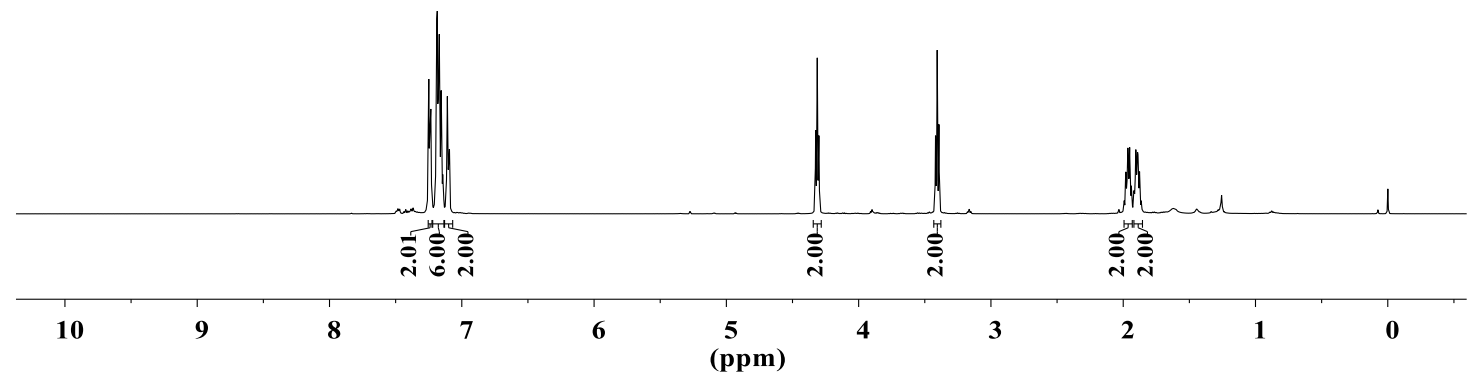

(500 MHz for ${ }^{1} \mathrm{H}$ NMR with $\mathrm{CDCl}_{3}$ as solvent)
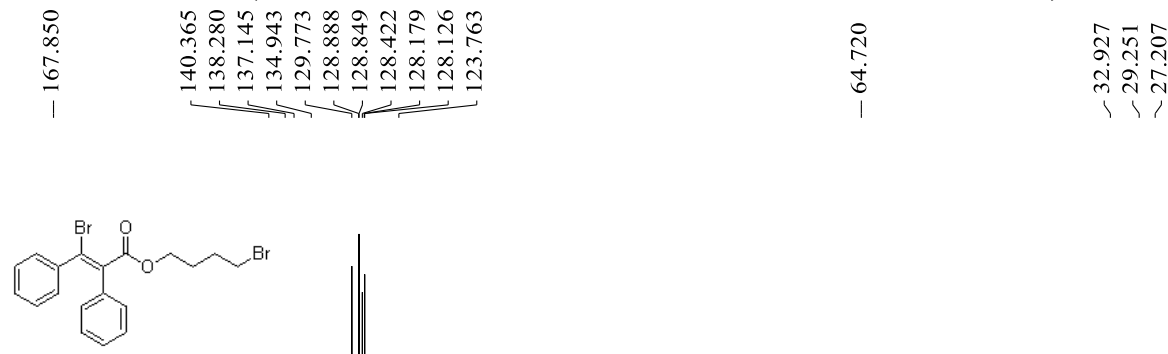

1a

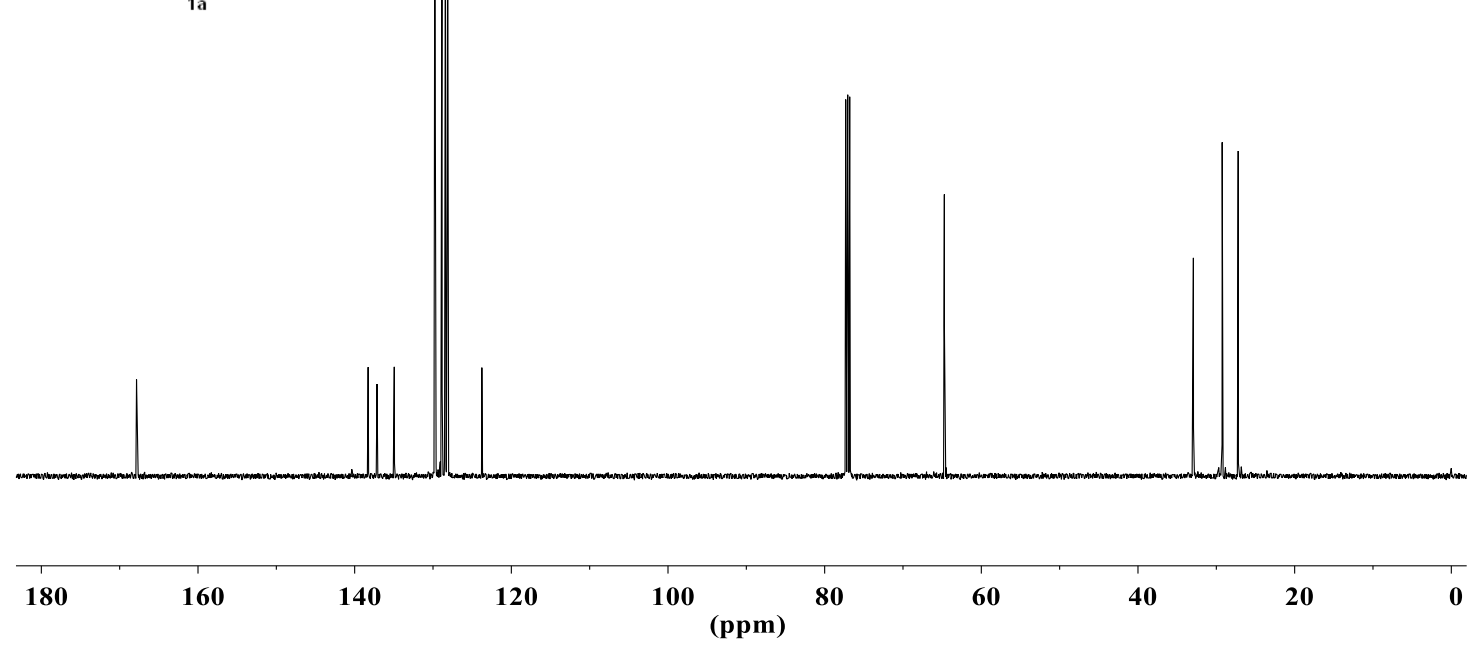

(125 MHz for ${ }^{13} \mathrm{C}$ NMR with $\mathrm{CDCl}_{3}$ as solvent) 


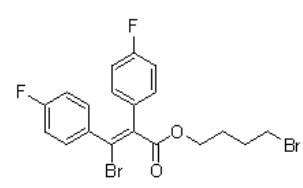

2a

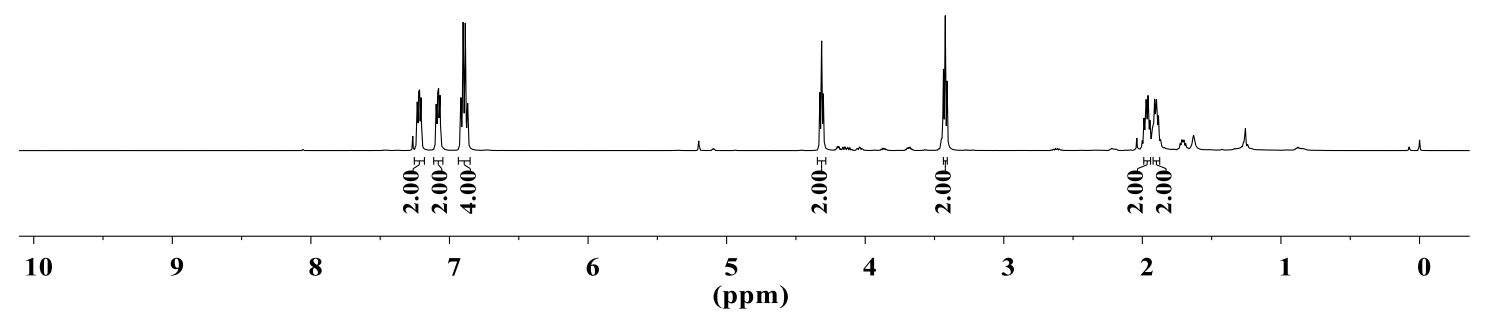

(500 $\mathrm{MHz}$ for ${ }^{1} \mathrm{H}$ NMR with $\mathrm{CDCl}_{3}$ as solvent)

ऊ

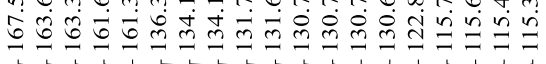

$\infty$
$\infty$
$\dot{b}$
$\dot{0}$
1

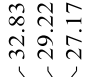

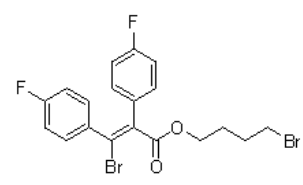

2a

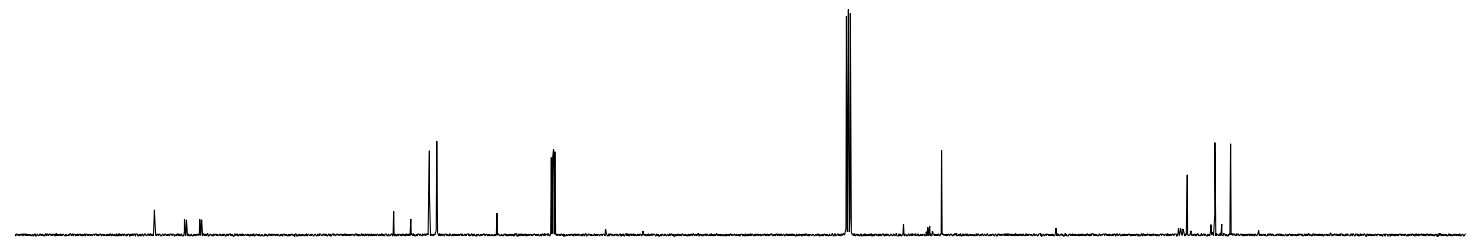

(125 MHz for ${ }^{13} \mathrm{C}$ NMR with $\mathrm{CDCl}_{3}$ as solvent) 


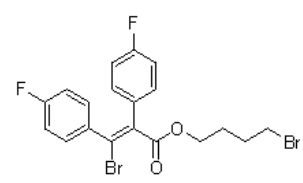

$2 a$

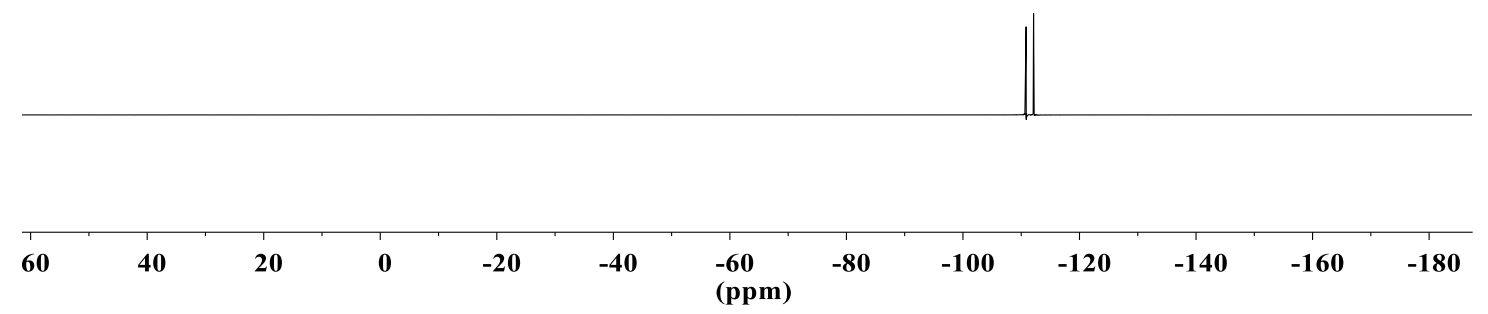

(500 $\mathrm{MHz}$ for ${ }^{1} \mathrm{H}$ NMR with $\mathrm{CDCl}_{3}$ as solvent)

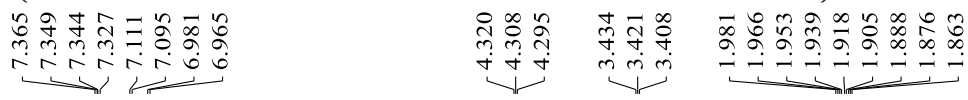

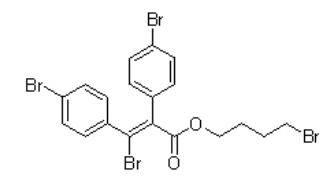

3a

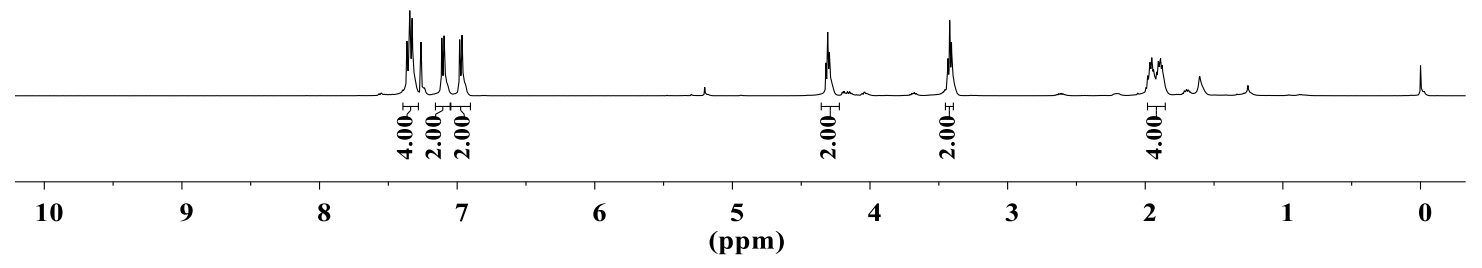

(500 $\mathrm{MHz}$ for ${ }^{1} \mathrm{H}$ NMR with $\mathrm{CDCl}_{3}$ as solvent) 


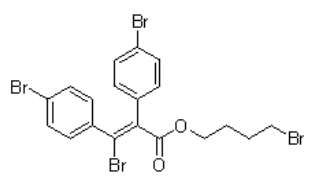

3a

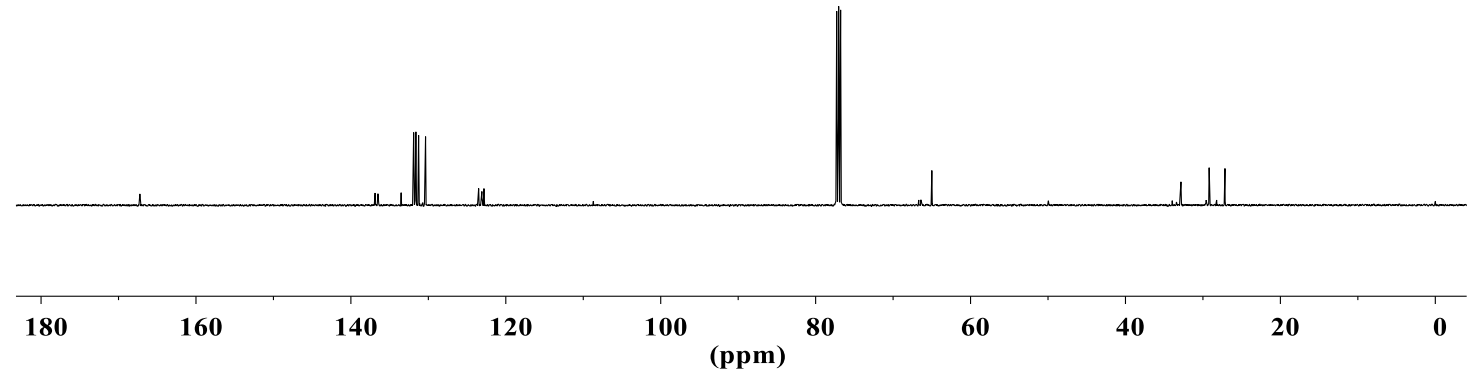

(125 $\mathrm{MHz}$ for ${ }^{13} \mathrm{C} \mathrm{NMR}$ with $\mathrm{CDCl}_{3}$ as solvent)

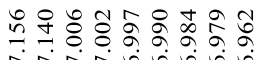

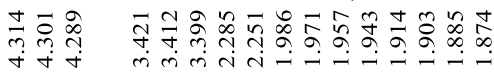

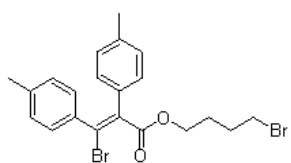

$4 a$

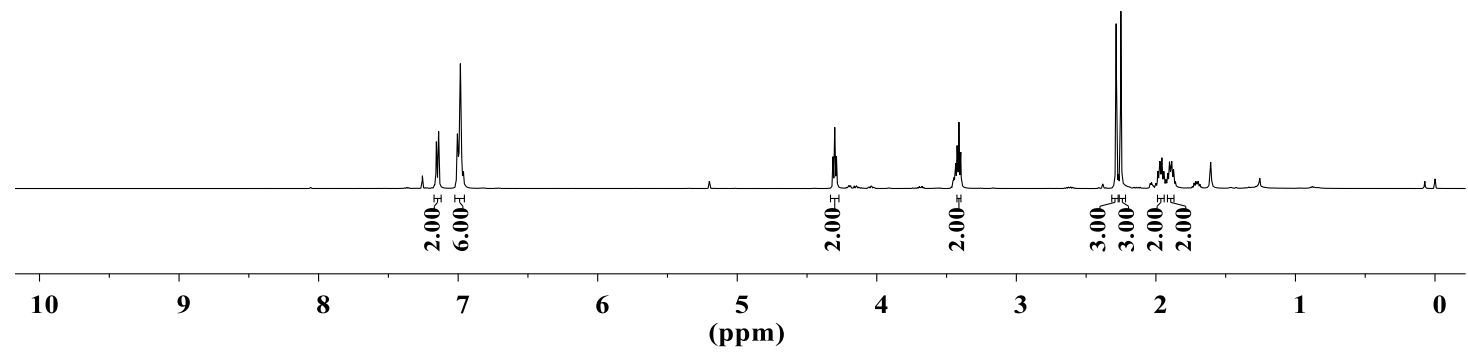

(500 MHz for ${ }^{1} \mathrm{H}$ NMR with $\mathrm{CDCl}_{3}$ as solvent) 

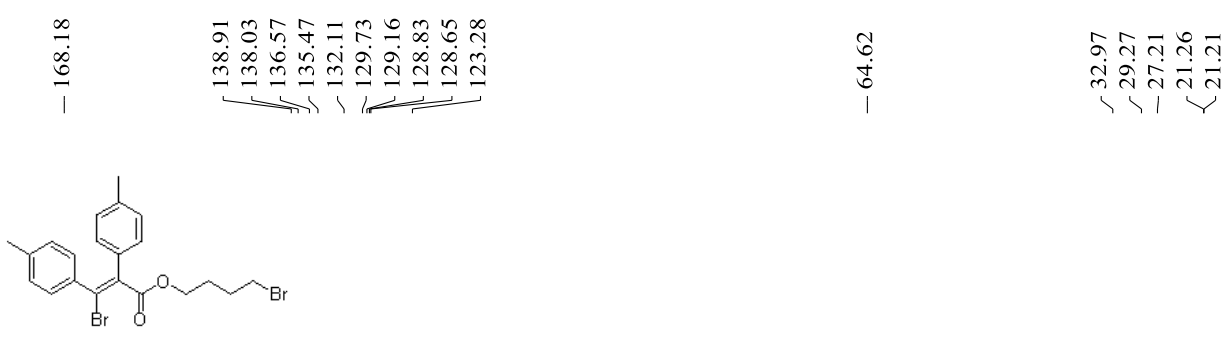

$4 a$

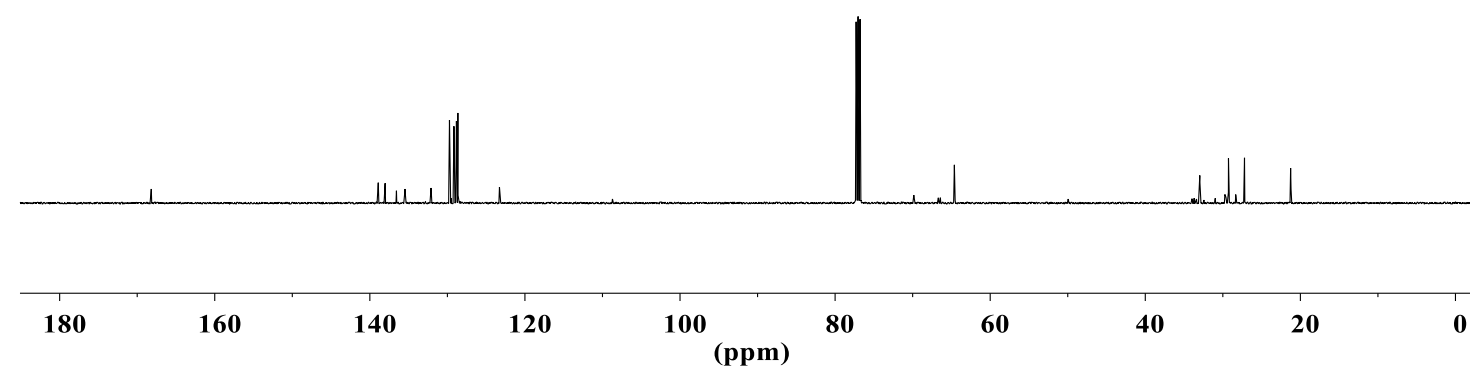

(125 MHz for ${ }^{13} \mathrm{C}$ NMR with $\mathrm{CDCl}_{3}$ as solvent)
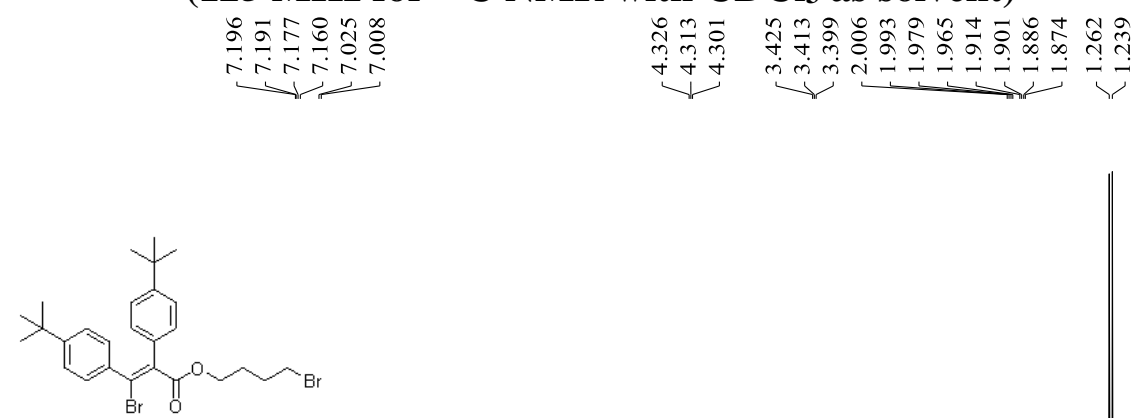

$5 a$

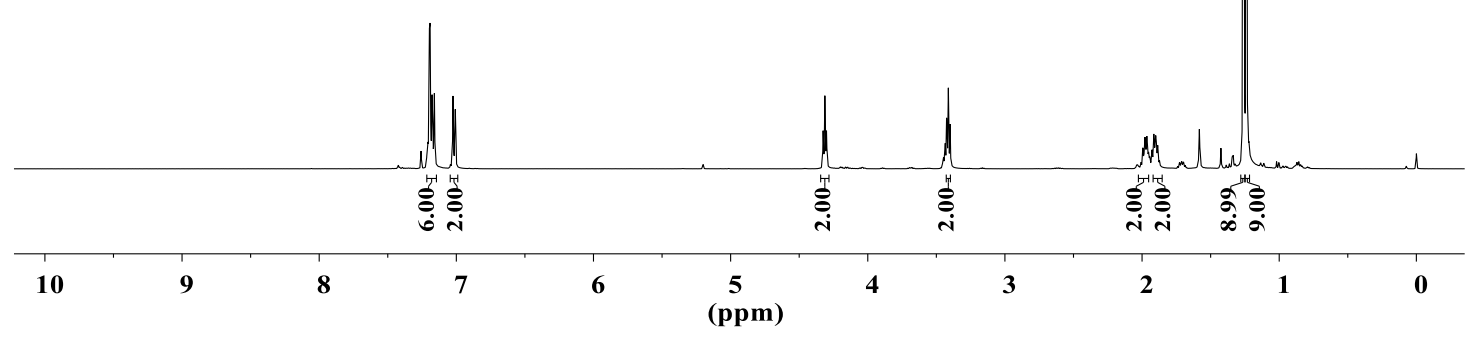

(500 MHz for ${ }^{1} \mathrm{H}$ NMR with $\mathrm{CDCl}_{3}$ as solvent) 


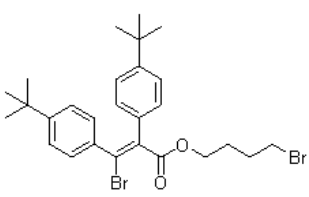

5 a

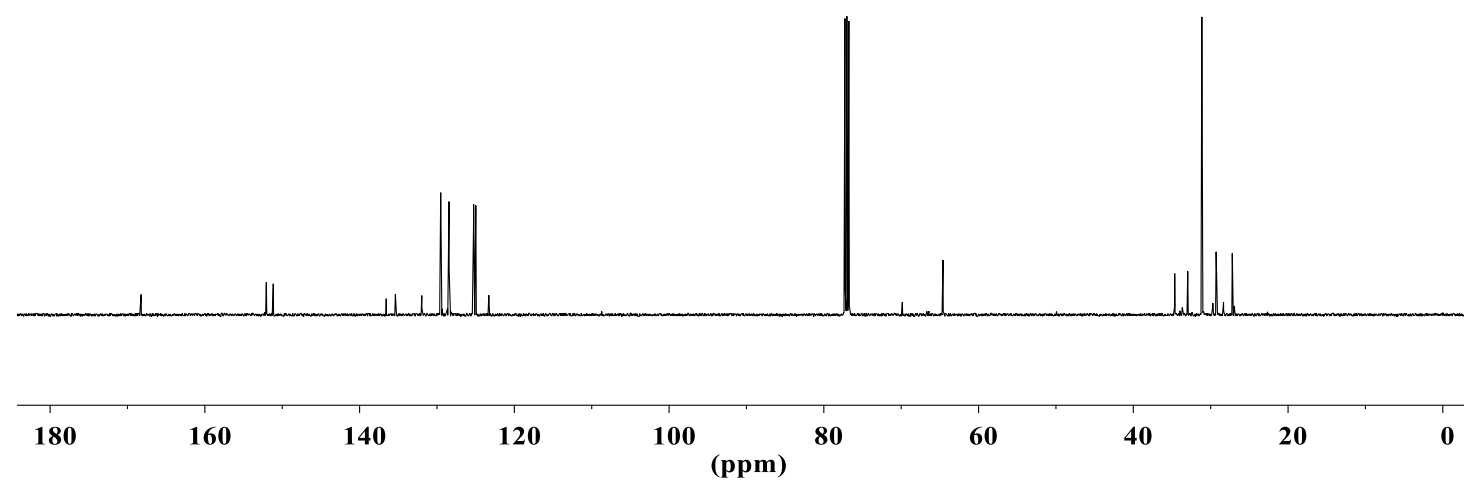

(125 MHz for ${ }^{13} \mathrm{C} \mathrm{NMR} \mathrm{with} \mathrm{CDCl}_{3}$ as solvent)

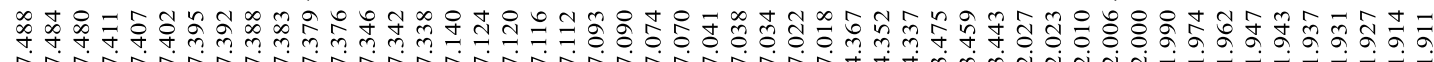

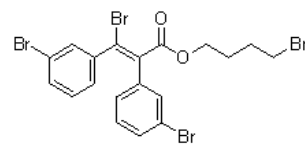

6 a

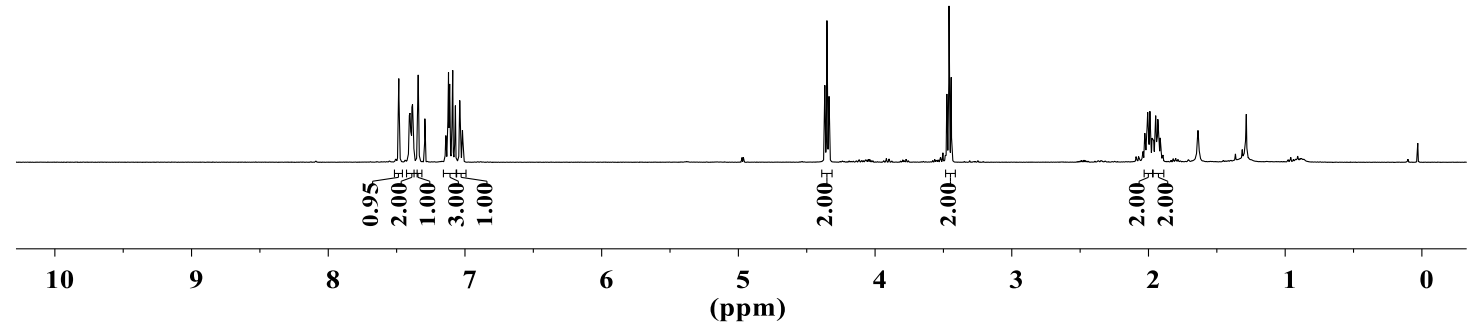

(500 MHz for ${ }^{1} \mathrm{H}$ NMR with $\mathrm{CDCl}_{3}$ as solvent) 


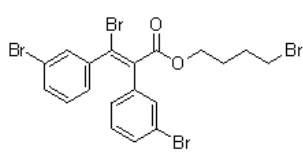

6 a

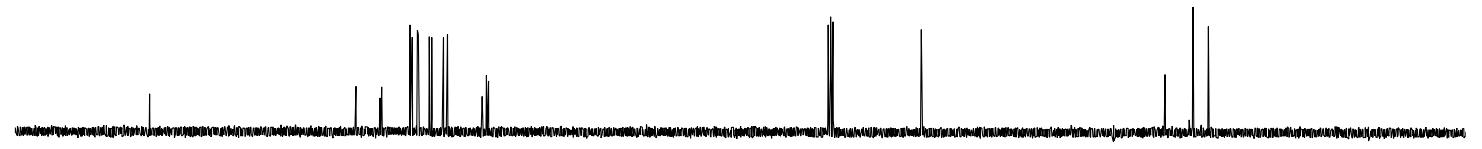

\begin{tabular}{llllllllll}
\hline 180 & 160 & 140 & 120 & 100 & 80 & 60 & 40 & 20 & 0
\end{tabular}

(125 MHz for ${ }^{13} \mathrm{C}$ NMR with $\mathrm{CDCl}_{3}$ as solvent)

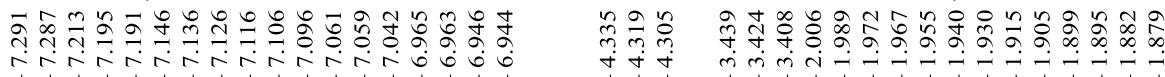
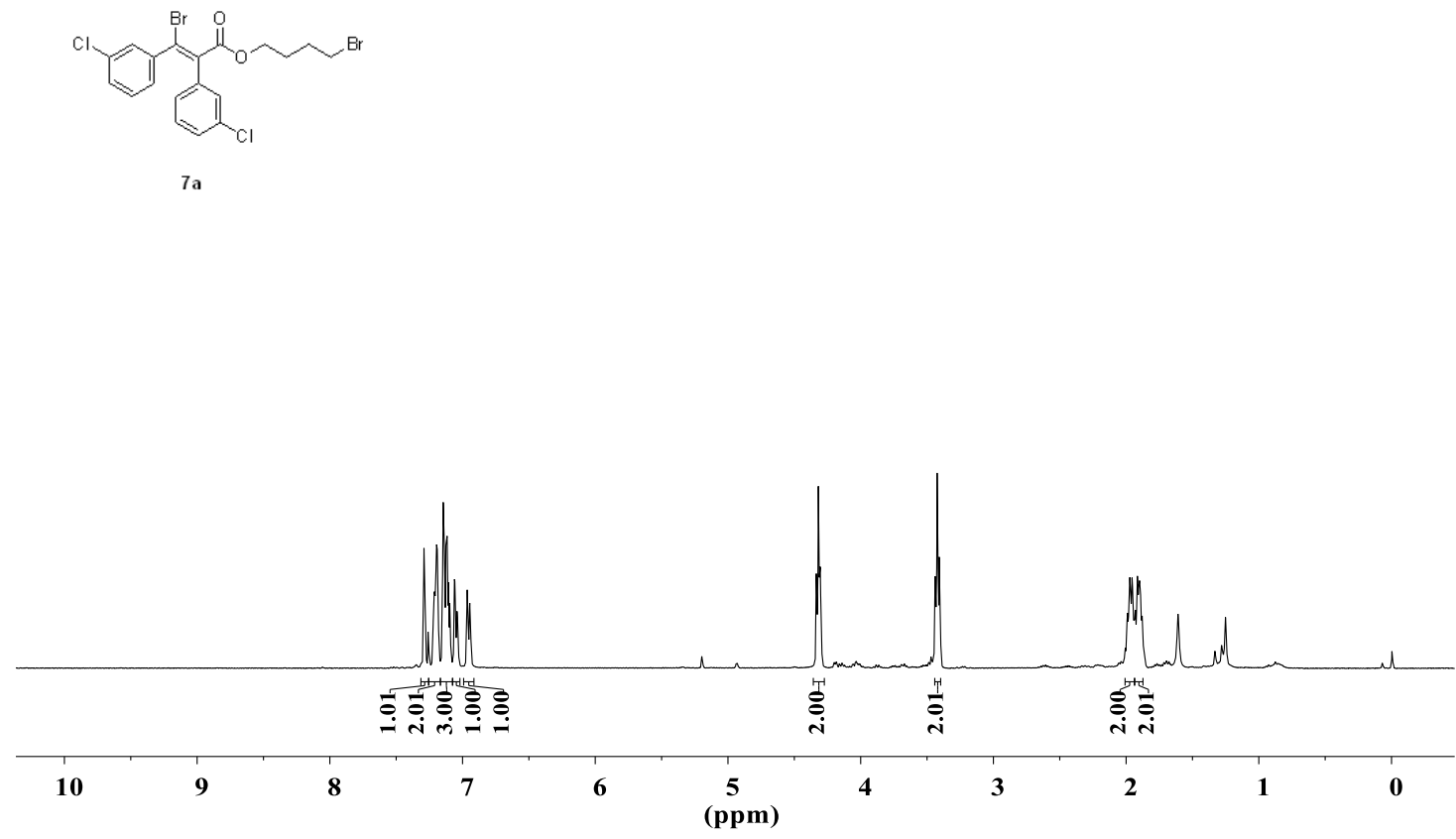

(500 MHz for ${ }^{1} \mathrm{H}$ NMR with $\mathrm{CDCl}_{3}$ as solvent) 


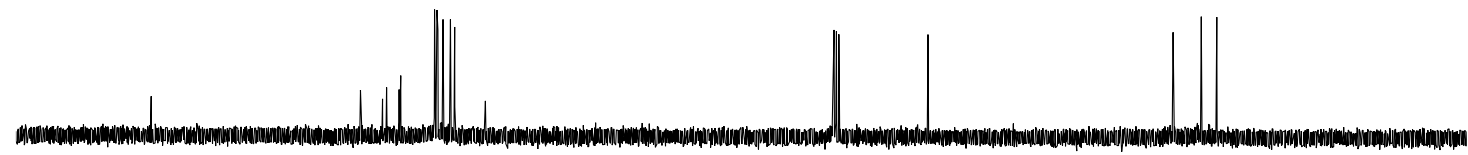

\begin{tabular}{llllllllll}
\hline 180 & 160 & 140 & 120 & 100 & 80 & 60 & 40 & 20 & 0
\end{tabular}

(125 MHz for ${ }^{13} \mathrm{C}$ NMR with $\mathrm{CDCl}_{3}$ as solvent)

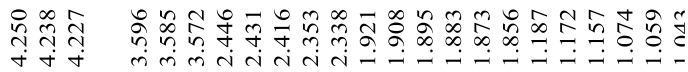<smiles>CCCCCOC(=O)C(Br)CC</smiles>

8 a

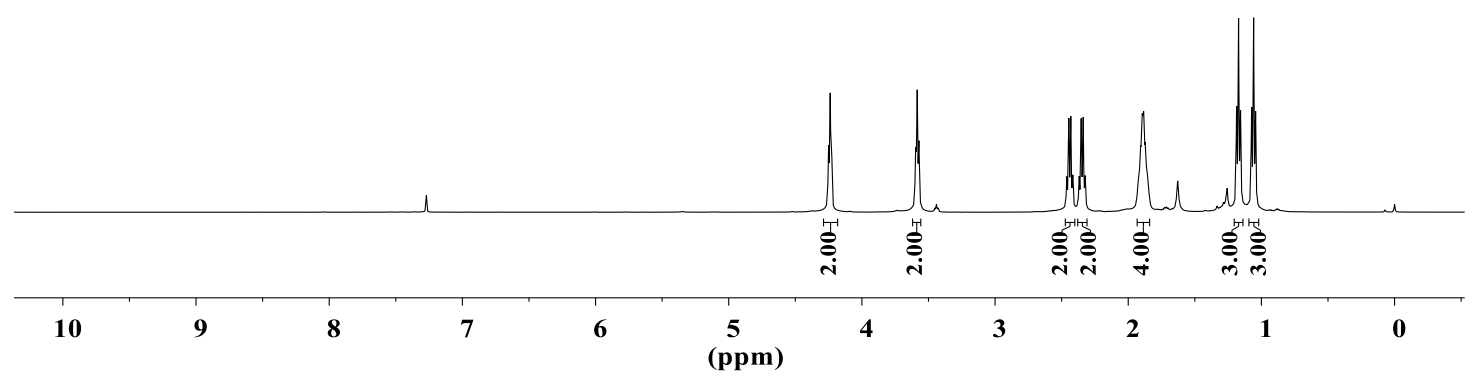

(500 MHz for ${ }^{1} \mathrm{H}$ NMR with $\mathrm{CDCl}_{3}$ as solvent) 


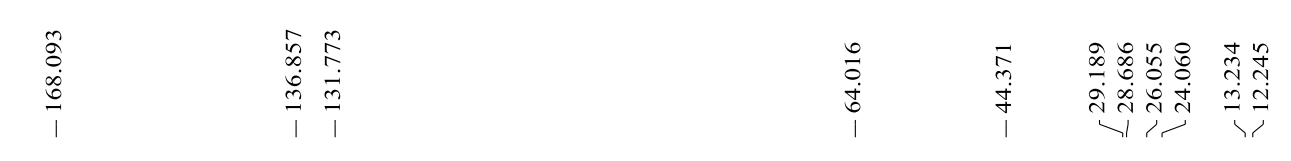

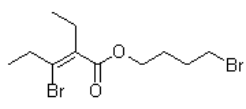

$8 \mathrm{a}$

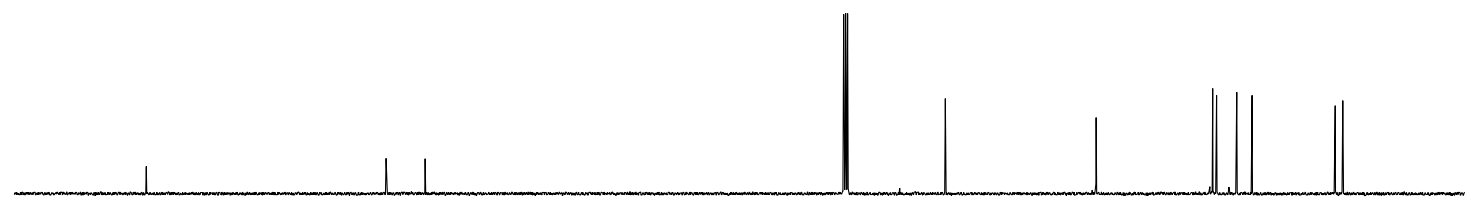

$\begin{array}{lllllllllll}180 & 160 & 140 & 120 & 100 & 80 & 60 & 40 & 20 & 0\end{array}$

(125 MHz for ${ }^{13} \mathrm{C}$ NMR with $\mathrm{CDCl}_{3}$ as solvent)

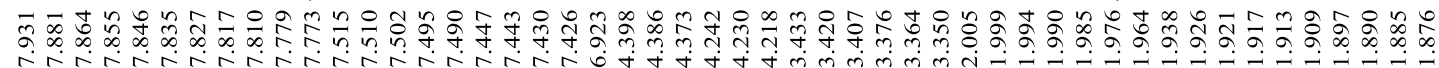
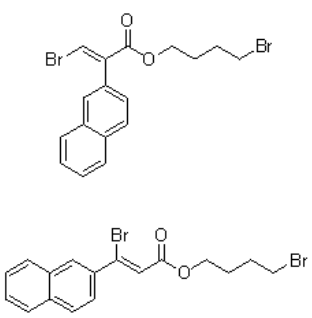

$9 a$ and $9 a^{\circ}$

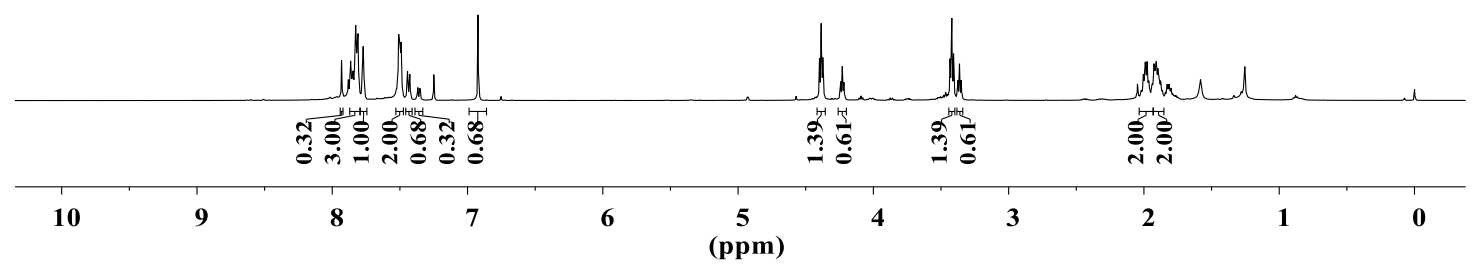

(500 MHz for ${ }^{1} \mathrm{H}$ NMR with $\mathrm{CDCl}_{3}$ as solvent) 

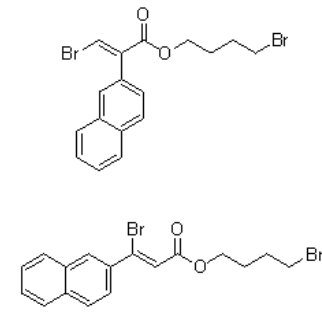

$9 \mathrm{a}$ and $9 \mathrm{a}^{\circ}$

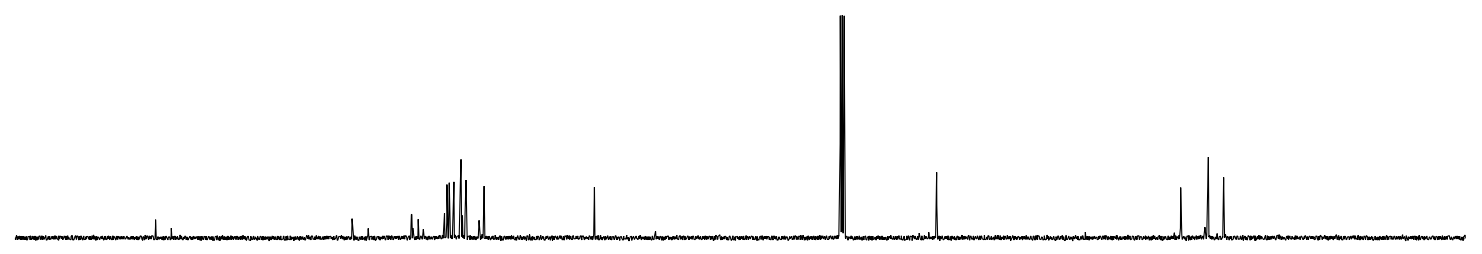

$180 \quad 160 \quad 140 \quad 120$

(ppm)

$80 \quad 60$

40

(125 $\mathrm{MHz}$ for ${ }^{13} \mathrm{C} \mathrm{NMR}$ with $\mathrm{CDCl}_{3}$ as solvent)

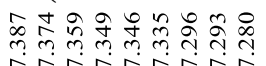

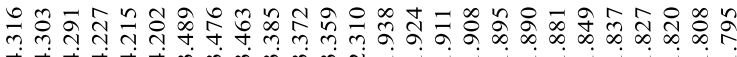

rarrarir

$\underbrace{.42}_{.4 .44 t}$<smiles>CCCCCOC(=O)/C(C)=C(\Br)c1ccccc1</smiles><smiles>CC(Br)=C(C(=O)OCCCCBr)c1ccccc1</smiles>

$10 \mathrm{a}$ and $10 \mathrm{a}^{\circ}$

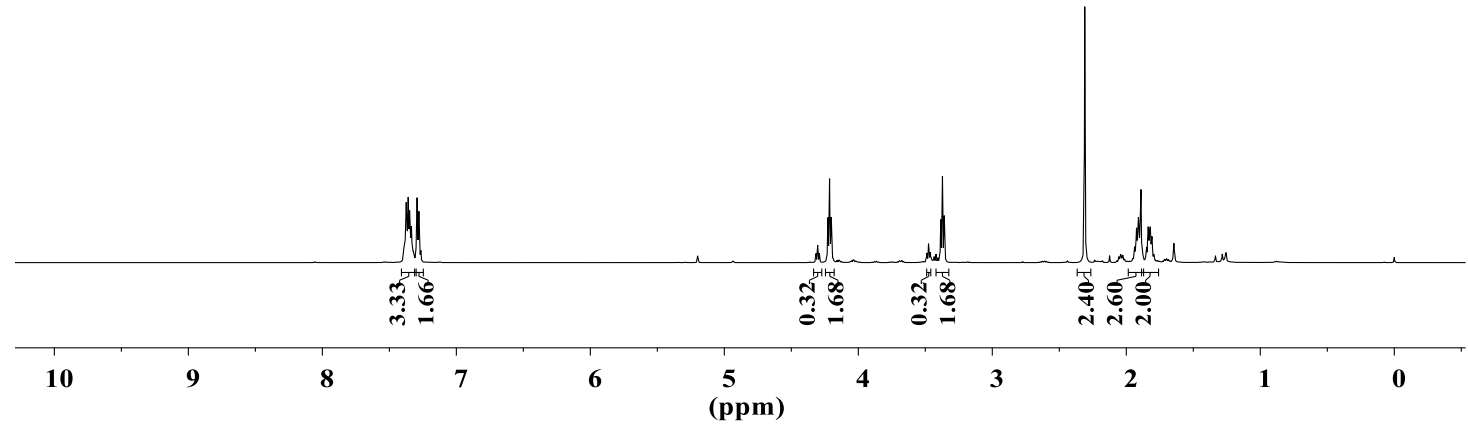

(500 MHz for ${ }^{1} \mathrm{H}$ NMR with $\mathrm{CDCl}_{3}$ as solvent) 
<smiles>CCCCCOC(=O)/C(C)=C(\Br)c1ccccc1</smiles><smiles>CC(Br)=C(C(=O)OCCCCBr)c1ccccc1</smiles>

$10 \mathrm{a}$ and $10 \mathrm{a}^{\circ}$

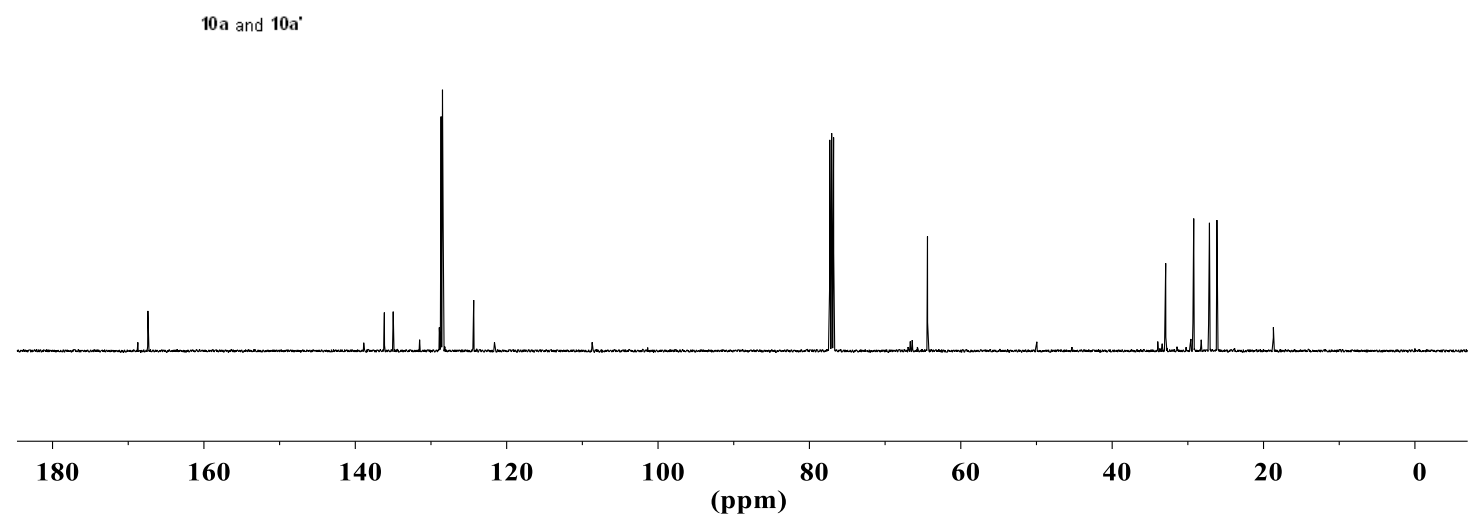

(125 MHz for ${ }^{13} \mathrm{C}$ NMR with $\mathrm{CDCl}_{3}$ as solvent)
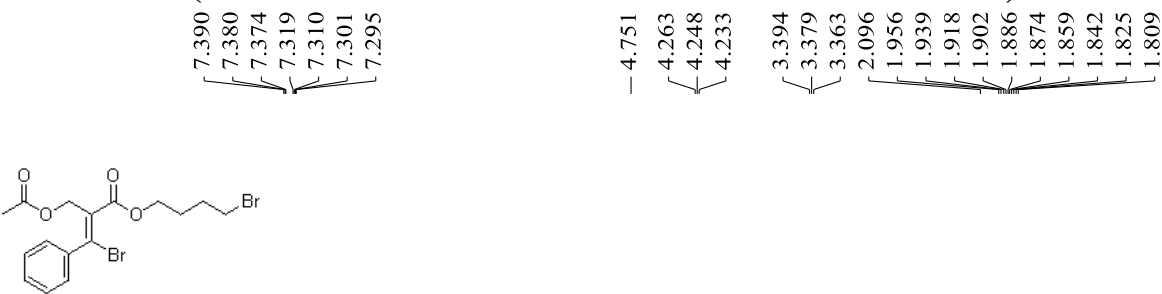

$11 \mathrm{a}$

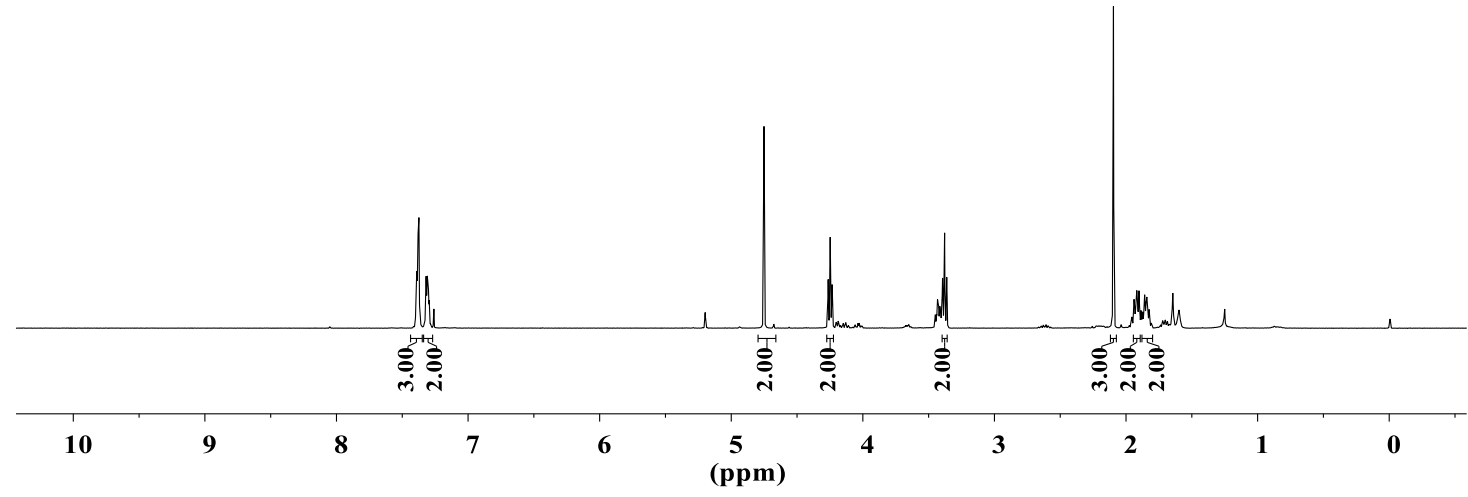

(500 MHz for ${ }^{1} \mathrm{H}$ NMR with $\mathrm{CDCl}_{3}$ as solvent) 


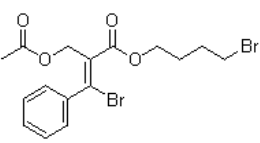

$11 \mathrm{a}$

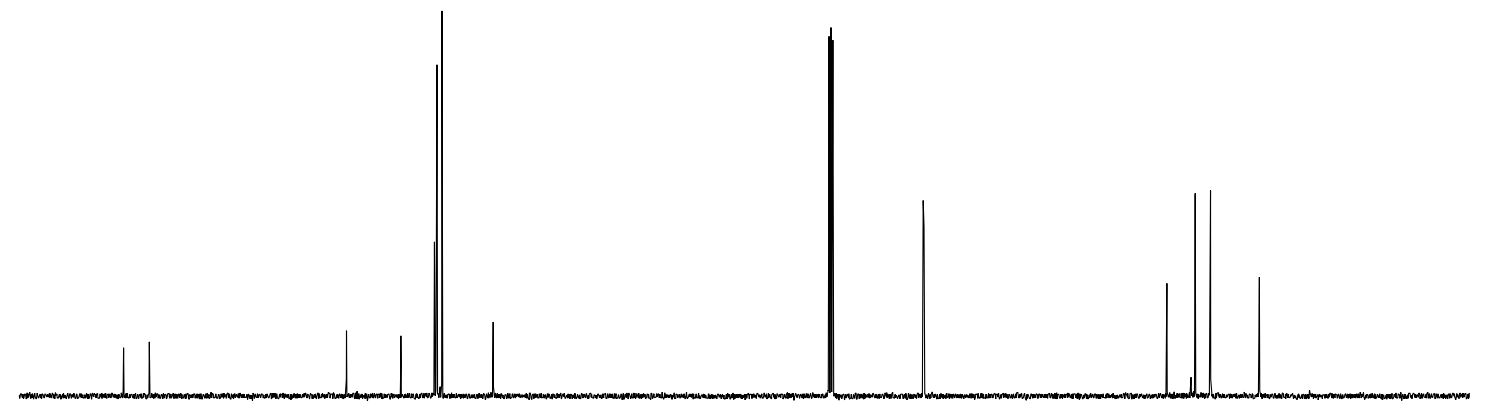

180

$160 \quad 140$
120

100

(ppm)

20

(125 MHz for ${ }^{13} \mathrm{C}$ NMR with $\mathrm{CDCl}_{3}$ as solvent)

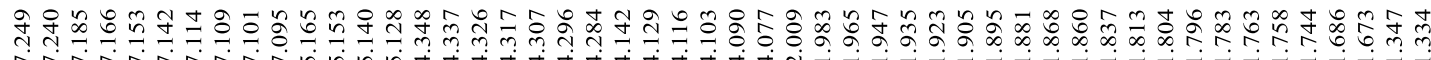

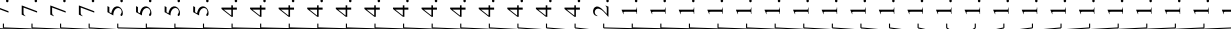

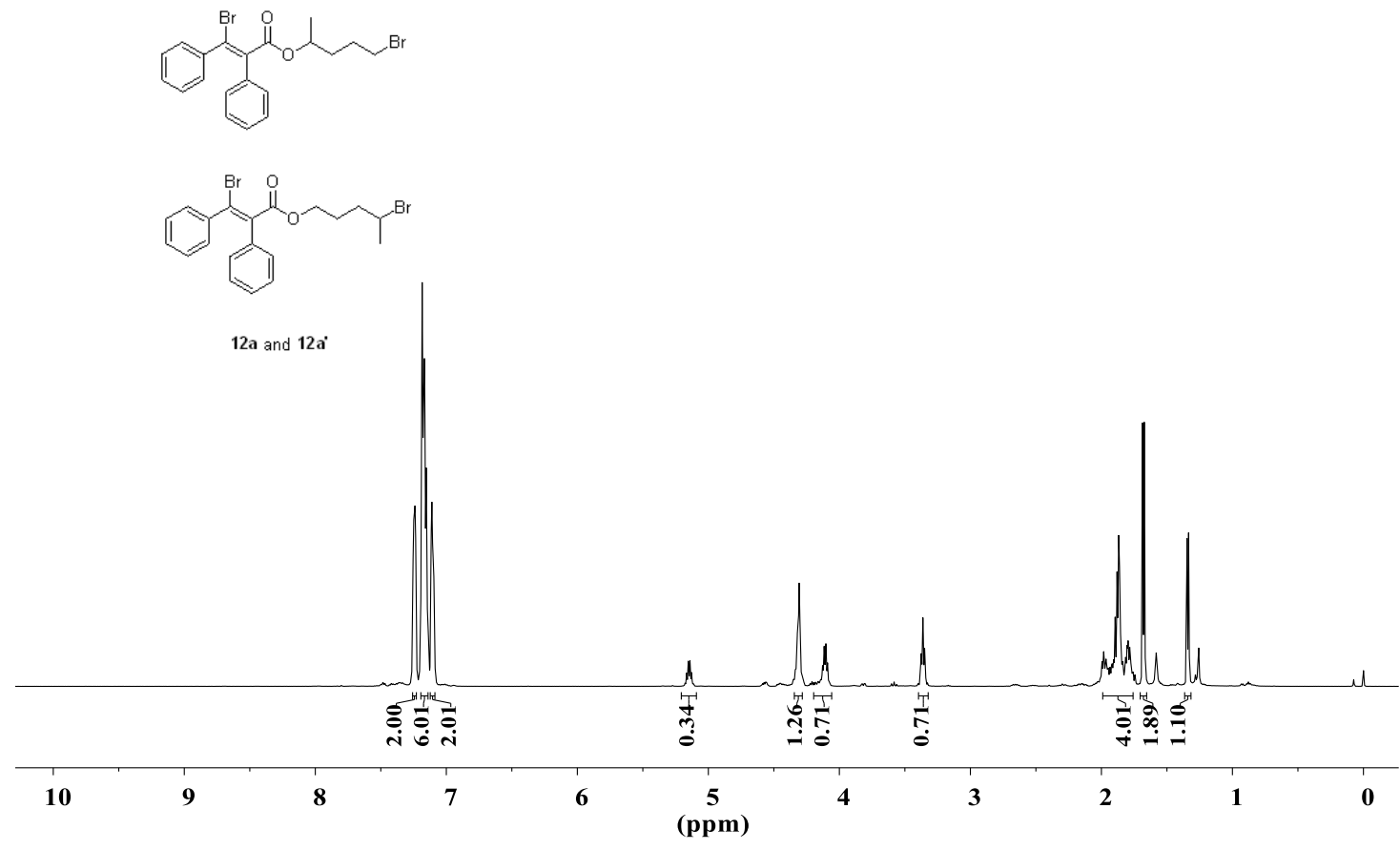

(500 MHz for ${ }^{1} \mathrm{H}$ NMR with $\mathrm{CDCl}_{3}$ as solvent) 
<smiles>CC(CCBr)OC(=O)C(=C(Br)c1ccccc1)c1ccccc1</smiles><smiles>CC(C)CCCOC(=O)C(Br)=C(c1ccccc1)c1ccccc1</smiles>

$12 \mathrm{a}$ and $12 \mathrm{a}$

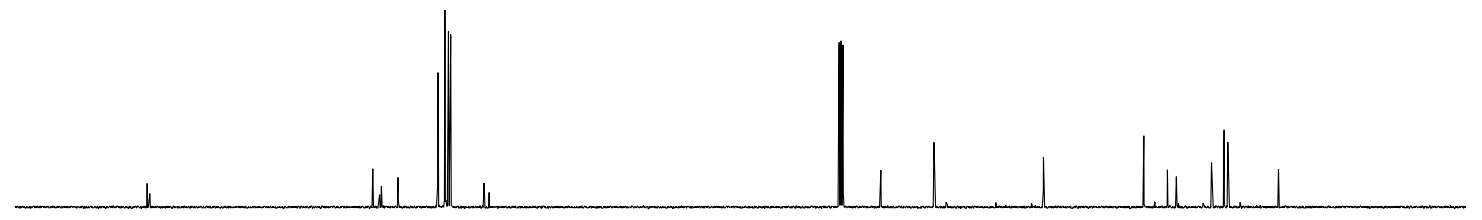

$\begin{array}{lllll}180 & 160 & 140 & 120 & 100\end{array}$

100

$80 \quad 60 \quad 40$

20 0

(125 MHz for ${ }^{13} \mathrm{C}$ NMR with $\mathrm{CDCl}_{3}$ as solvent)

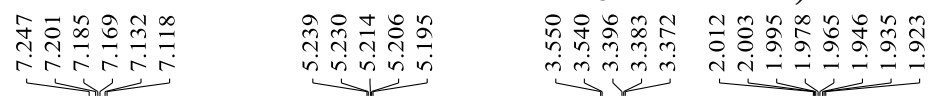

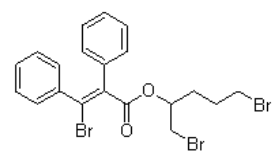

$13 \mathrm{a}$

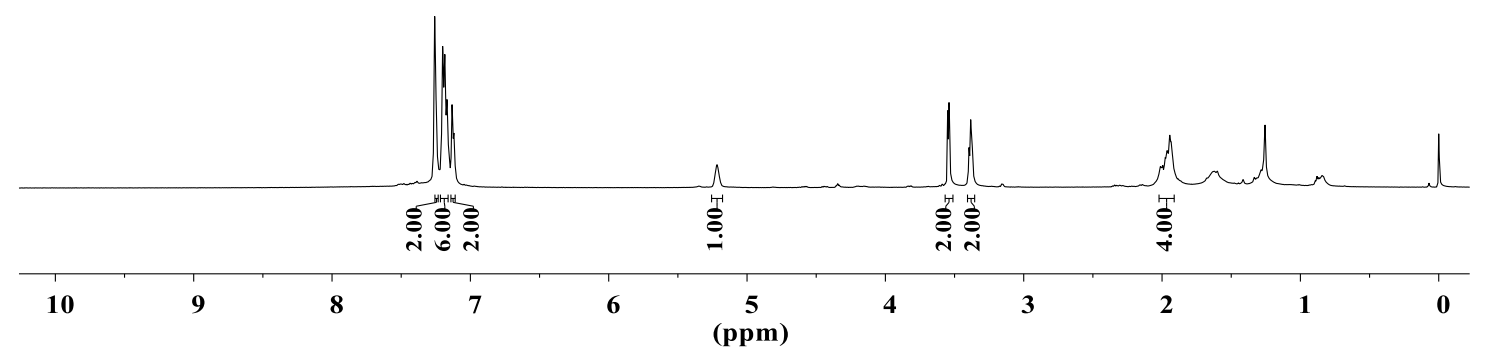

(500 MHz for ${ }^{1} \mathrm{H}$ NMR with $\mathrm{CDCl}_{3}$ as solvent) 

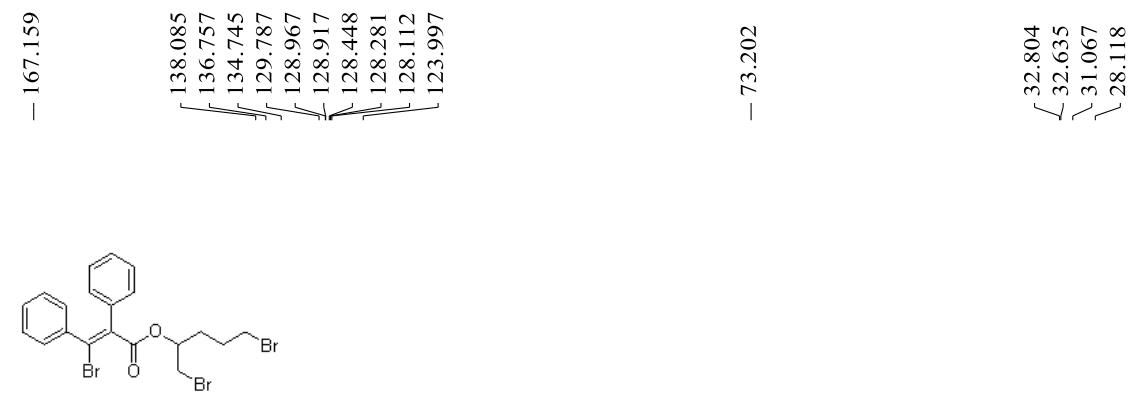

13a

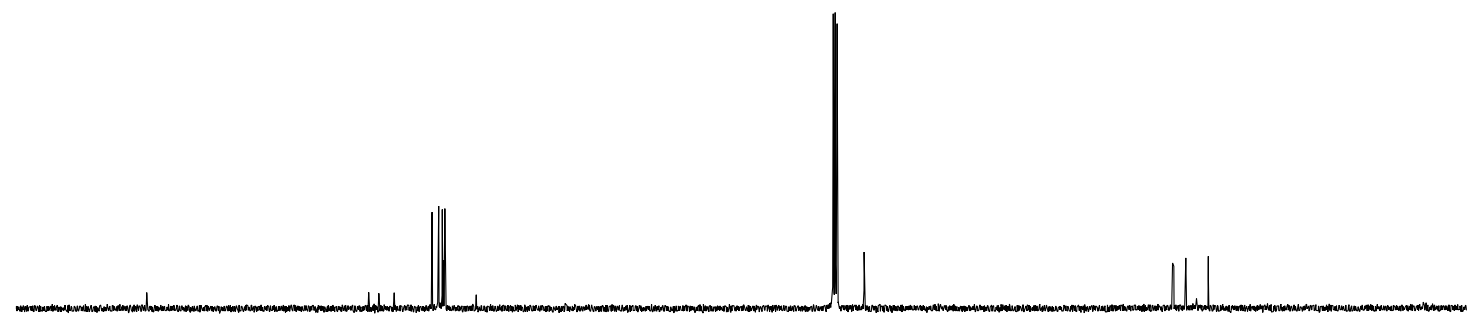

\begin{tabular}{|c|c|c|c|c|c|c|c|c|}
\hline 180 & 160 & 140 & 120 & 100 & 80 & 60 & 40 & 20 \\
\hline
\end{tabular}

(125 MHz for ${ }^{13} \mathrm{C}$ NMR with $\mathrm{CDCl}_{3}$ as solvent)

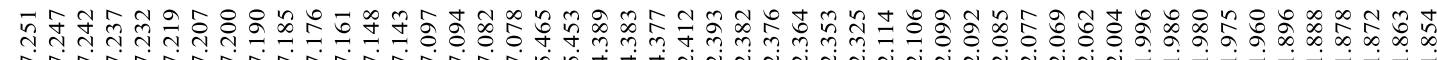

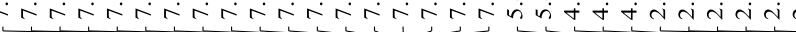

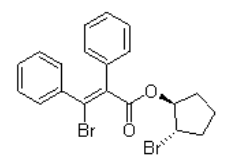

14a

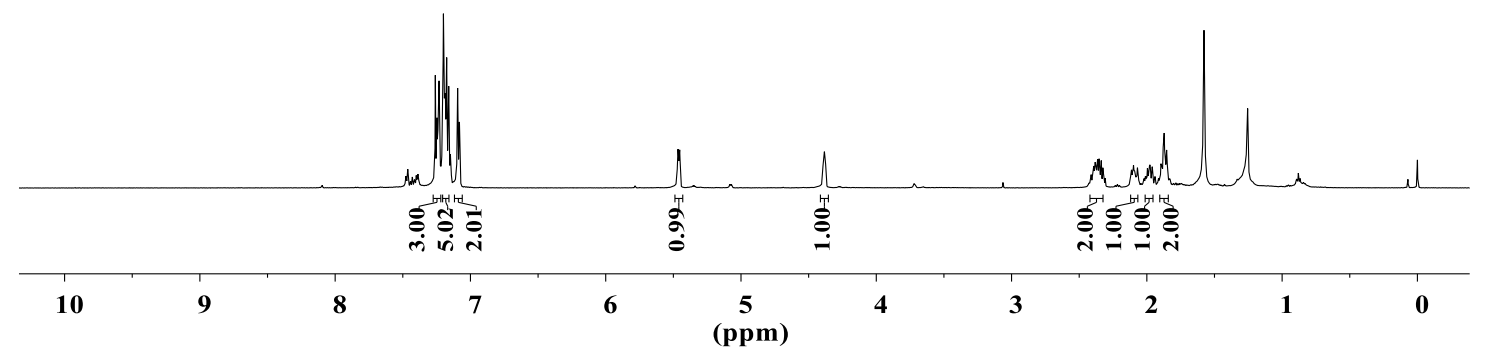

(500 MHz for ${ }^{1} \mathrm{H}$ NMR with $\mathrm{CDCl}_{3}$ as solvent) 


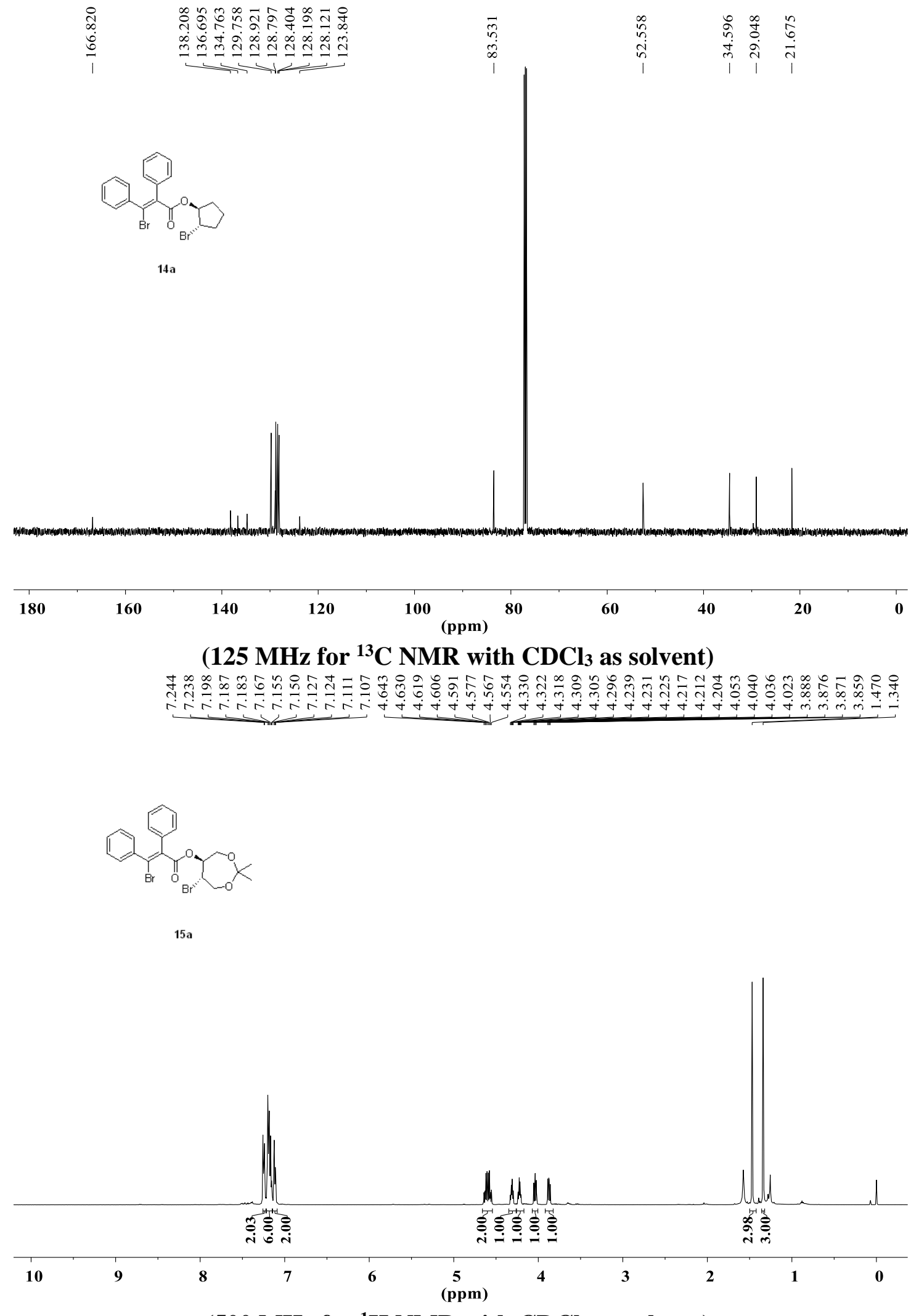

(500 MHz for ${ }^{1} \mathrm{H}$ NMR with $\mathrm{CDCl}_{3}$ as solvent) 


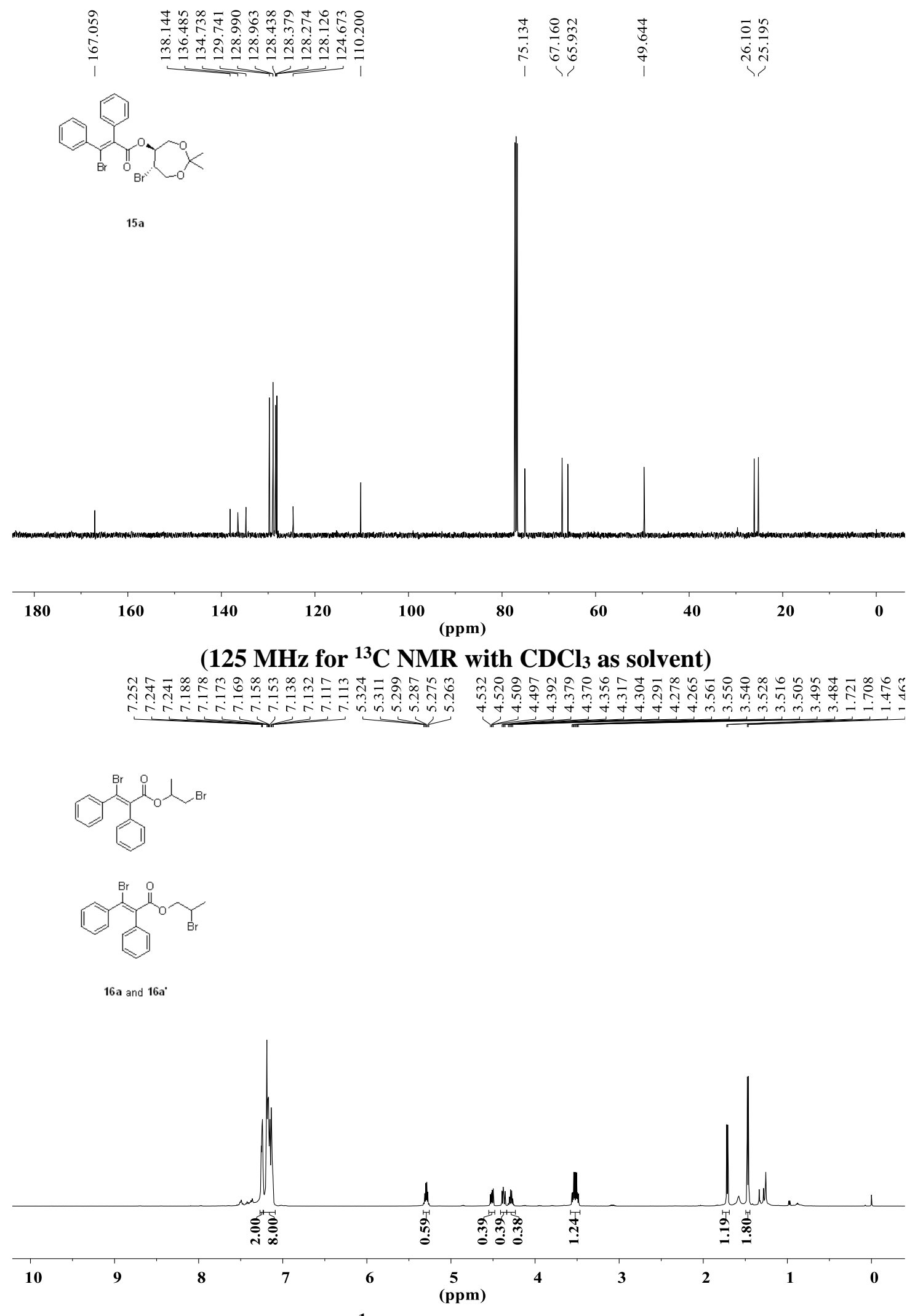

(500 MHz for ${ }^{1} \mathrm{H}$ NMR with $\mathrm{CDCl}_{3}$ as solvent) 


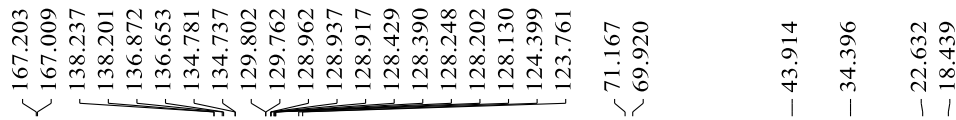

1)

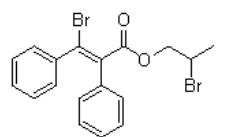

$16 \mathrm{a}$ and $16 \mathrm{a}^{\circ}$

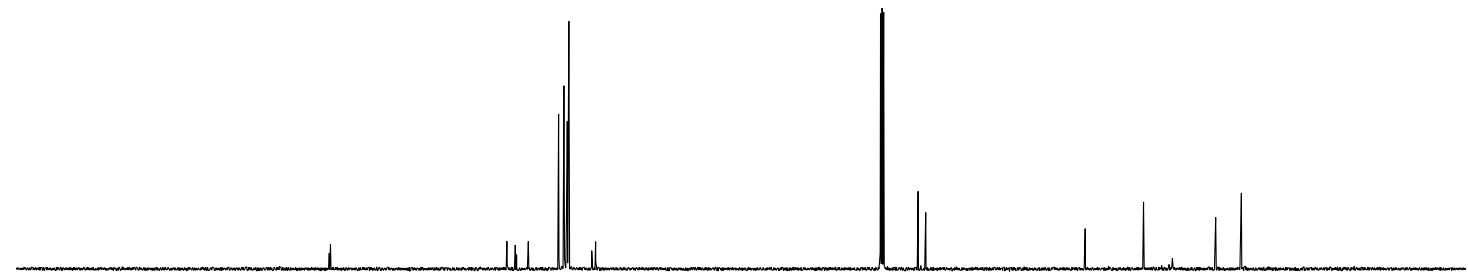

200

180

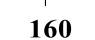

140

120

100

80

60

40

20

(125 MHz for ${ }^{13} \mathrm{C}$ NMR with $\mathrm{CDCl}_{3}$ as solvent)

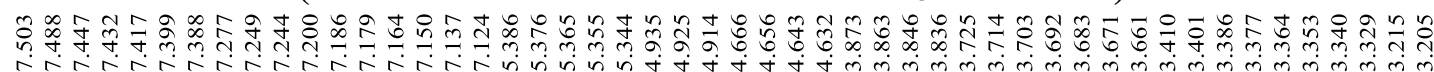

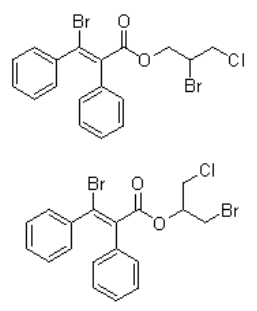

$17 \mathrm{a}$ and $17 \mathrm{a}^{\circ}$

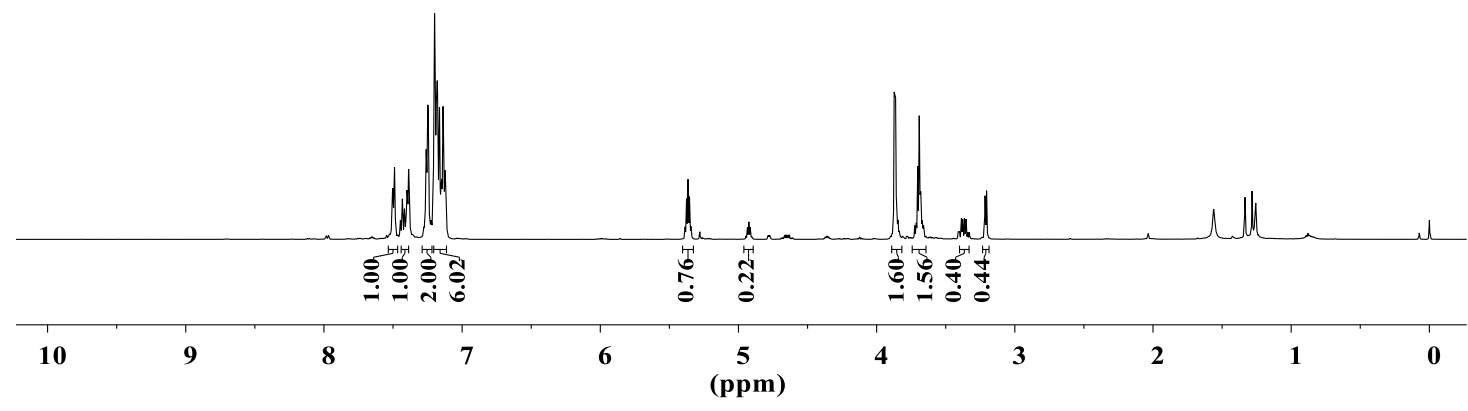

(500 MHz for ${ }^{1} \mathrm{H}$ NMR with $\mathrm{CDCl}_{3}$ as solvent) 

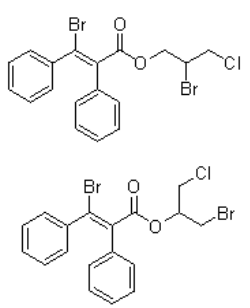

$17 \mathrm{a}$ and $17 \mathrm{a}$

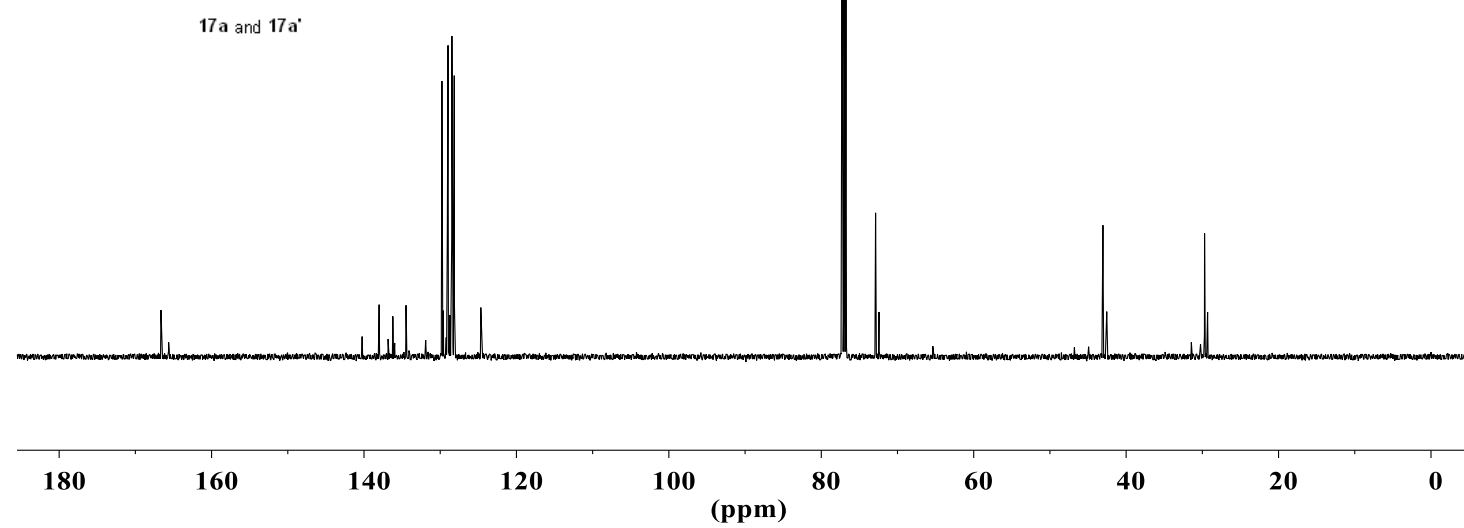

(125 MHz for ${ }^{13} \mathrm{C}$ NMR with $\mathrm{CDCl}_{3}$ as solvent)

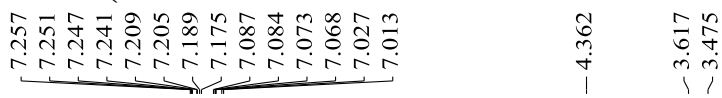

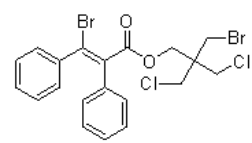

18 a

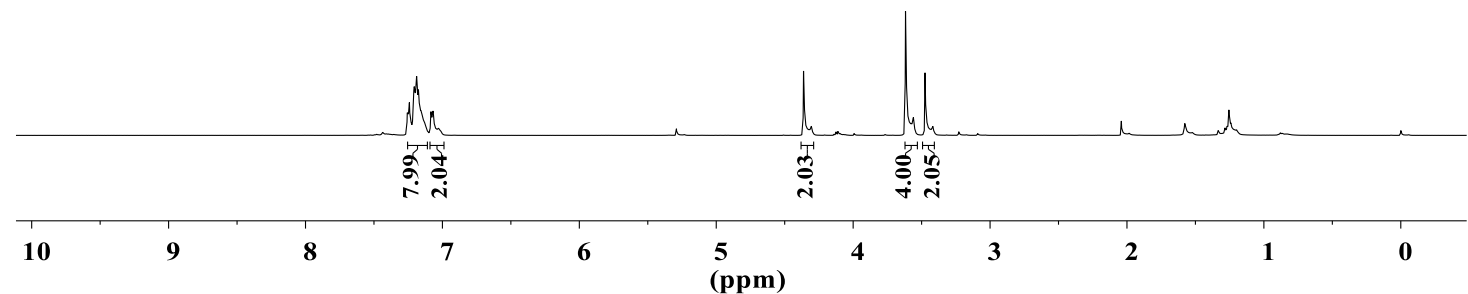

(500 $\mathrm{MHz}$ for ${ }^{1} \mathrm{H}$ NMR with $\mathrm{CDCl}_{3}$ as solvent) 


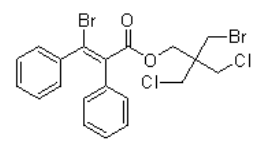

$18 \mathrm{a}$

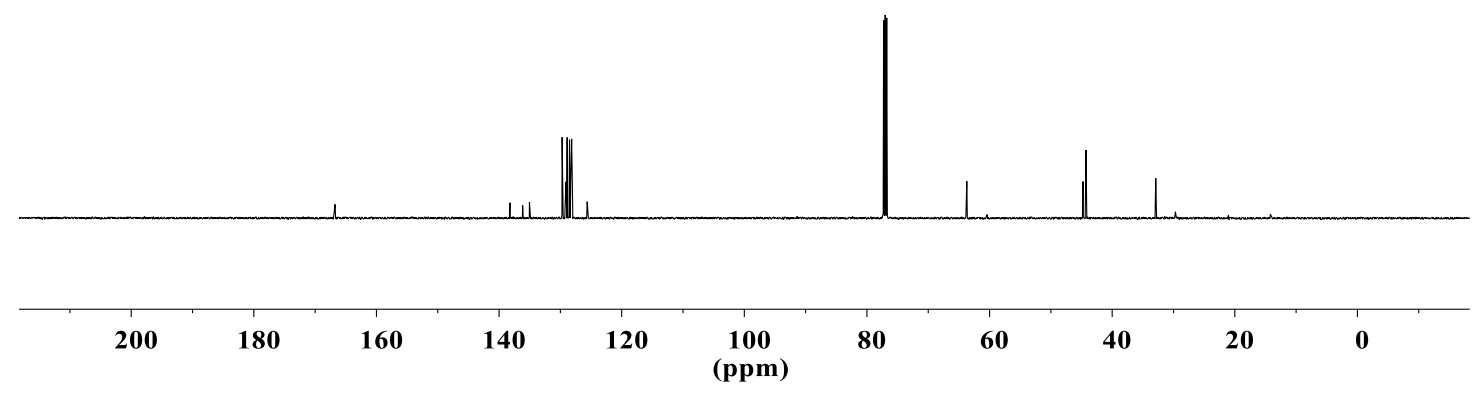

(125 $\mathrm{MHz}$ for ${ }^{13} \mathrm{C} \mathrm{NMR}$ with $\mathrm{CDCl}_{3}$ as solvent)

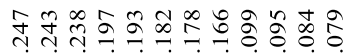

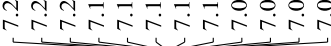

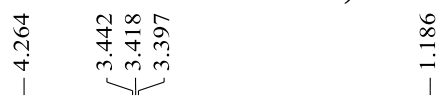

$\overbrace{\mathrm{Br}}^{\mathrm{B}}$

19a

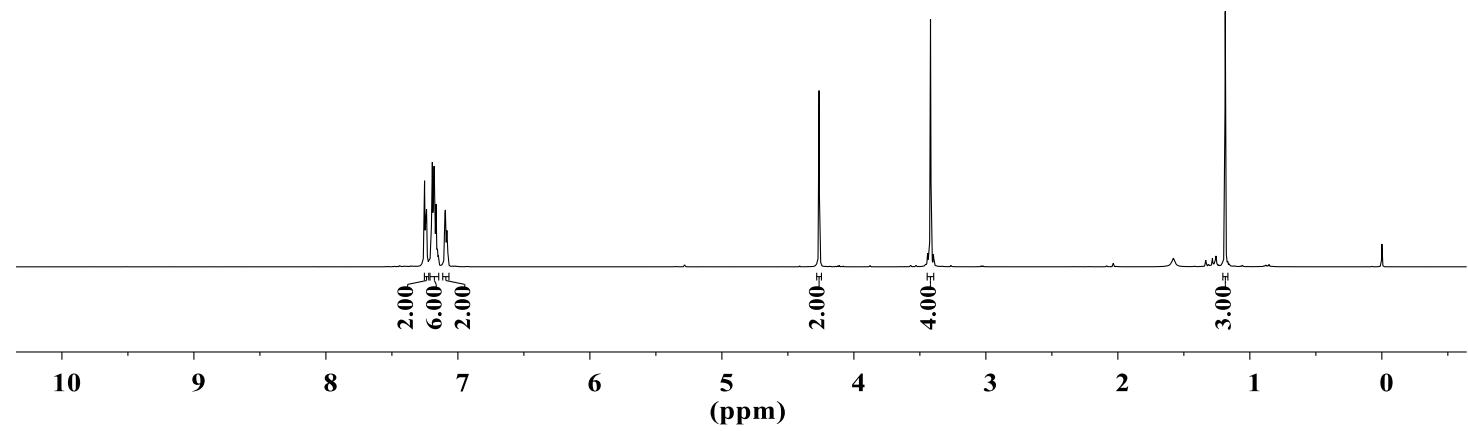

(500 MHz for ${ }^{1} \mathrm{H} \mathrm{NMR}$ with $\mathrm{CDCl}_{3}$ as solvent) 


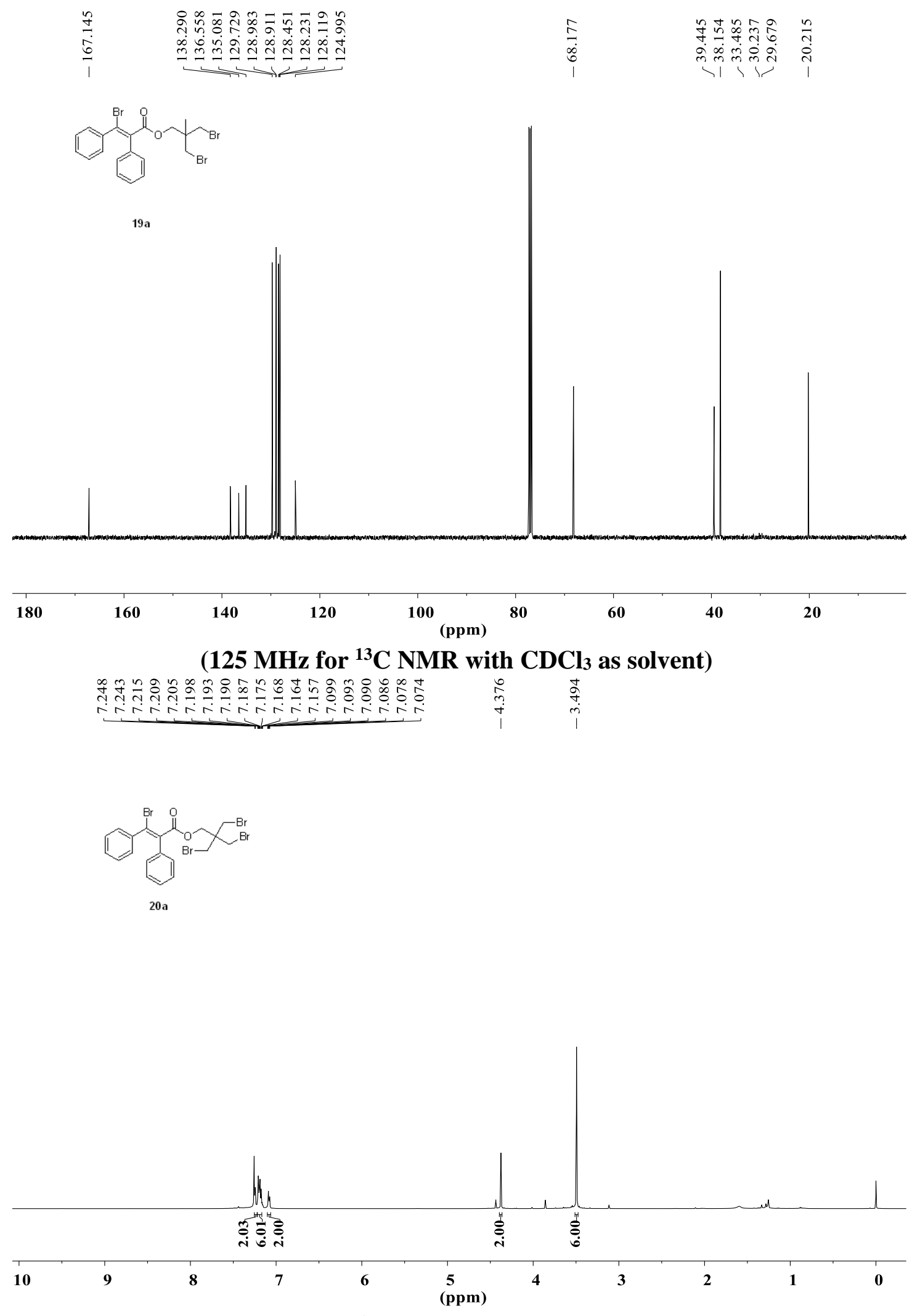

(500 MHz for ${ }^{1} \mathrm{H}$ NMR with $\mathrm{CDCl}_{3}$ as solvent) 


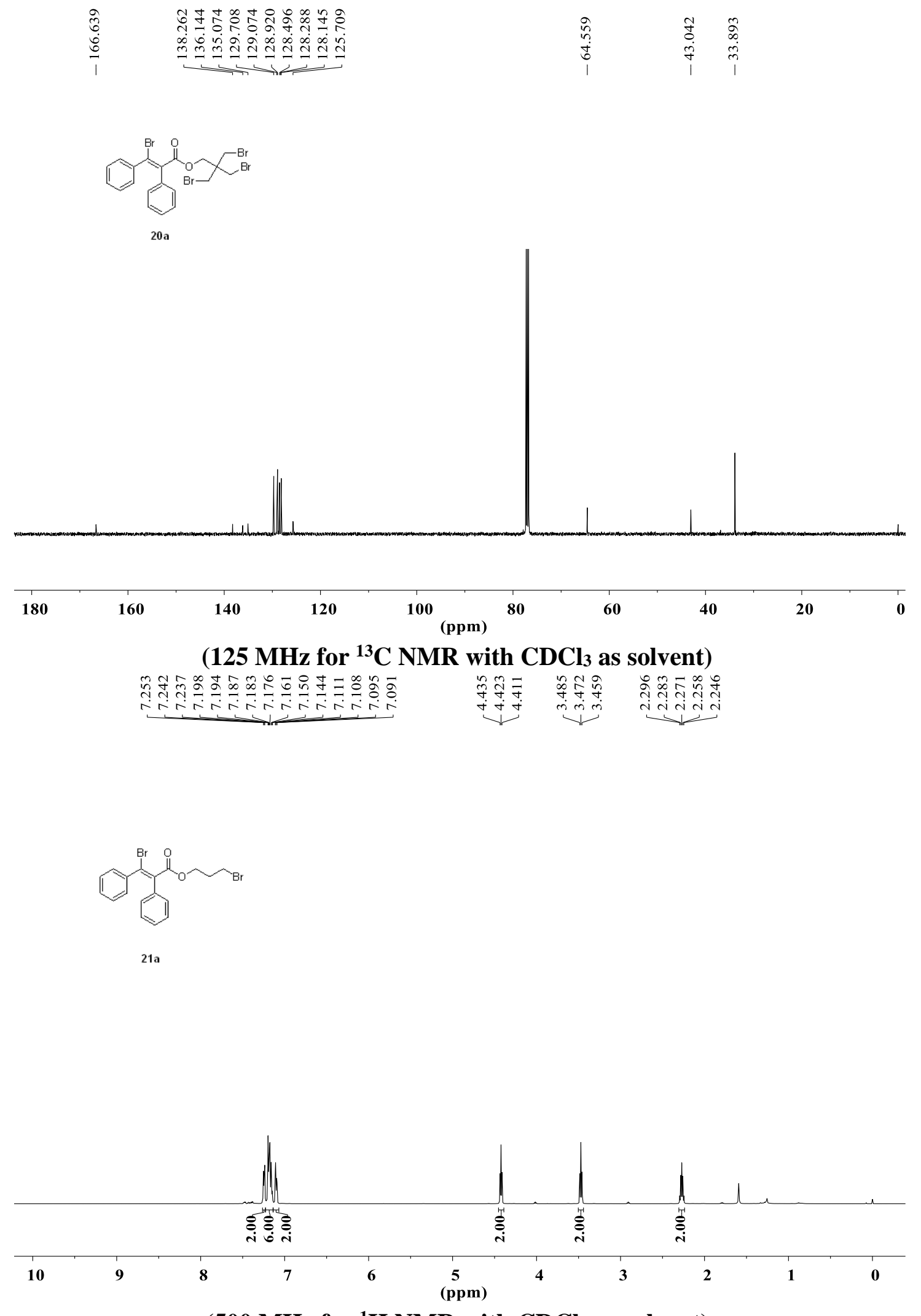

(500 $\mathrm{MHz}$ for ${ }^{1} \mathrm{H}$ NMR with $\mathrm{CDCl}_{3}$ as solvent) 


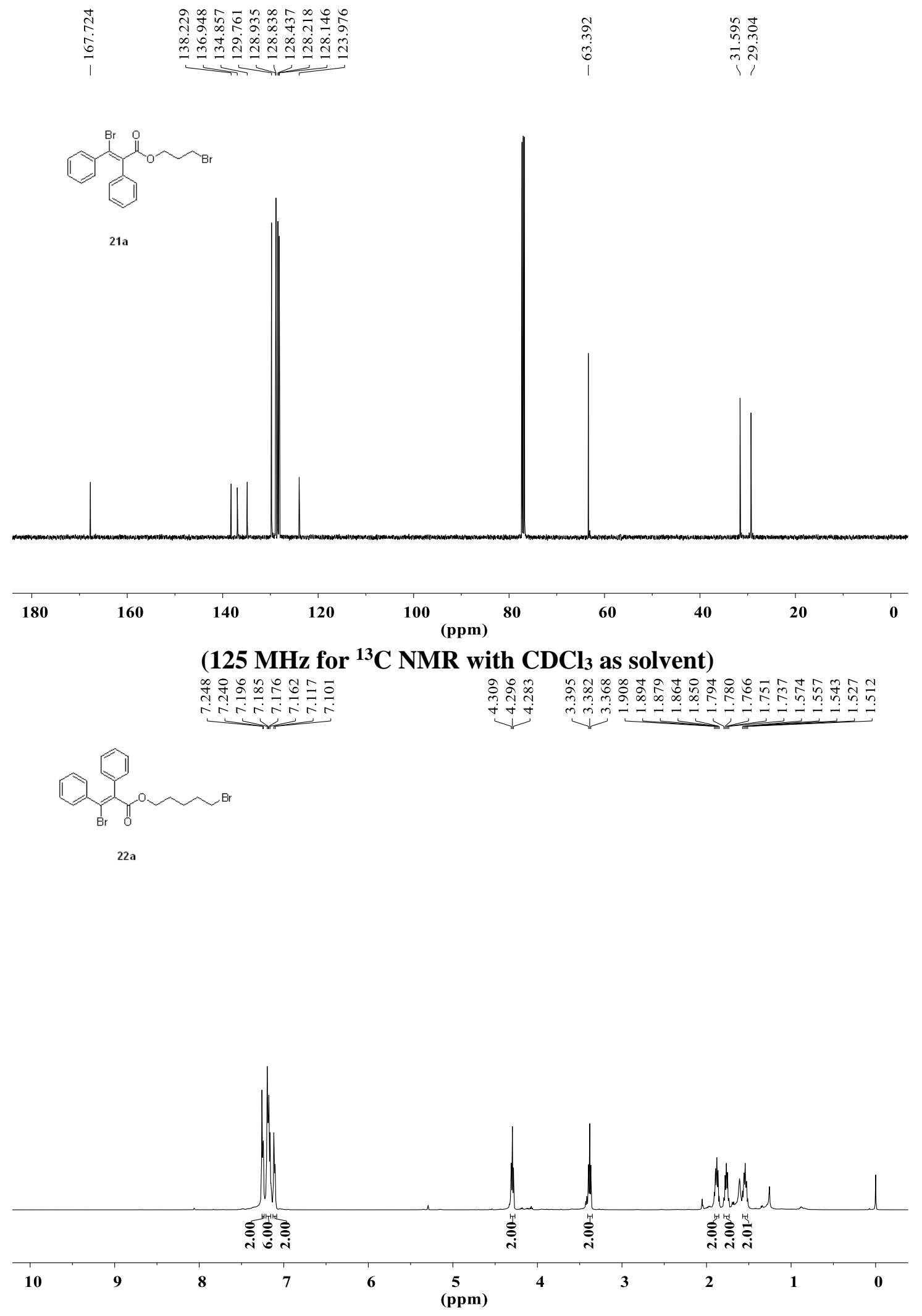

(500 $\mathrm{MHz}$ for ${ }^{1} \mathrm{H}$ NMR with $\mathrm{CDCl}_{3}$ as solvent) 


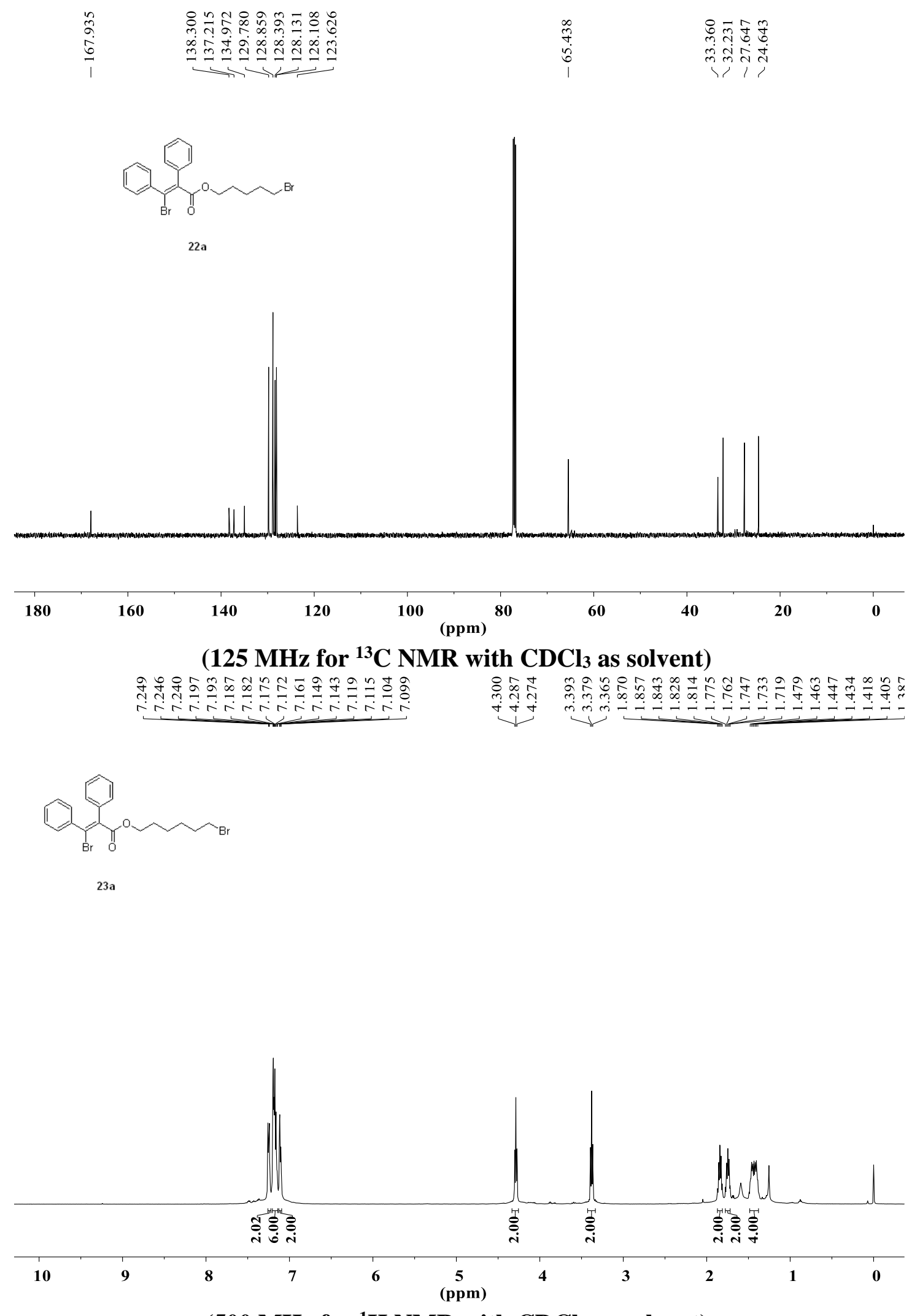

(500 MHz for ${ }^{1} \mathrm{H}$ NMR with $\mathrm{CDCl}_{3}$ as solvent) 


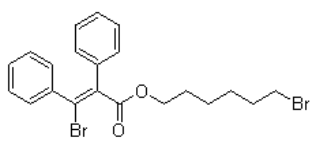

23
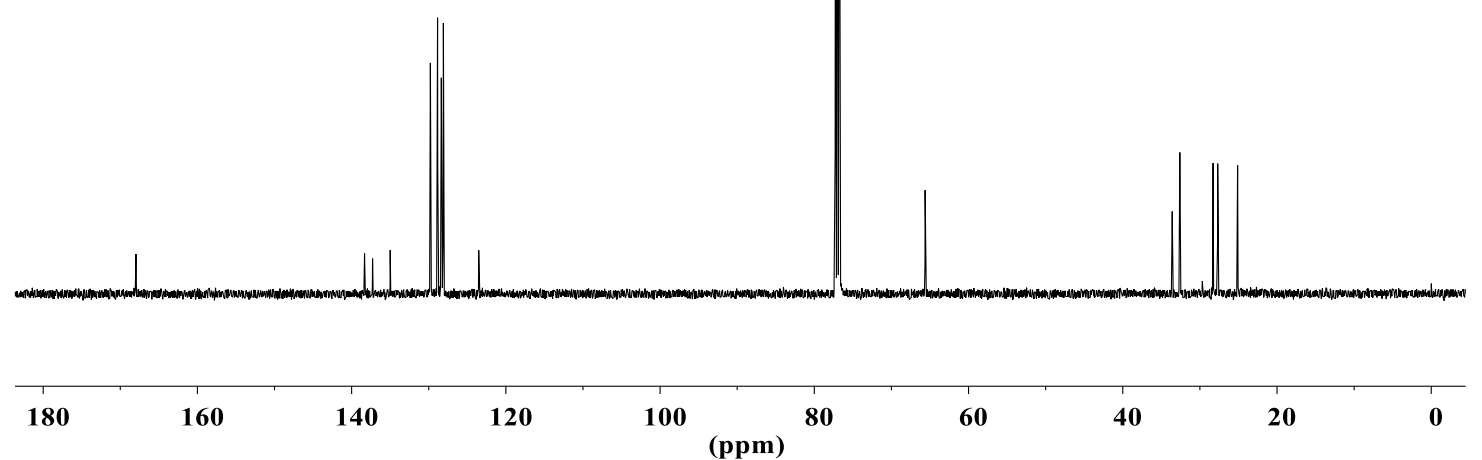

(125 MHz for ${ }^{13} \mathrm{C} \mathrm{NMR}$ with $\mathrm{CDCl}_{3}$ as solvent)

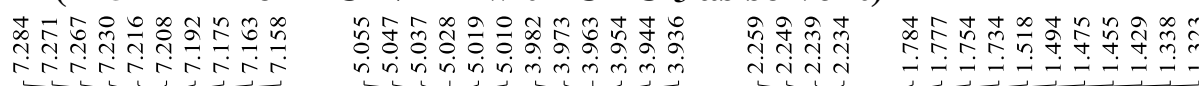
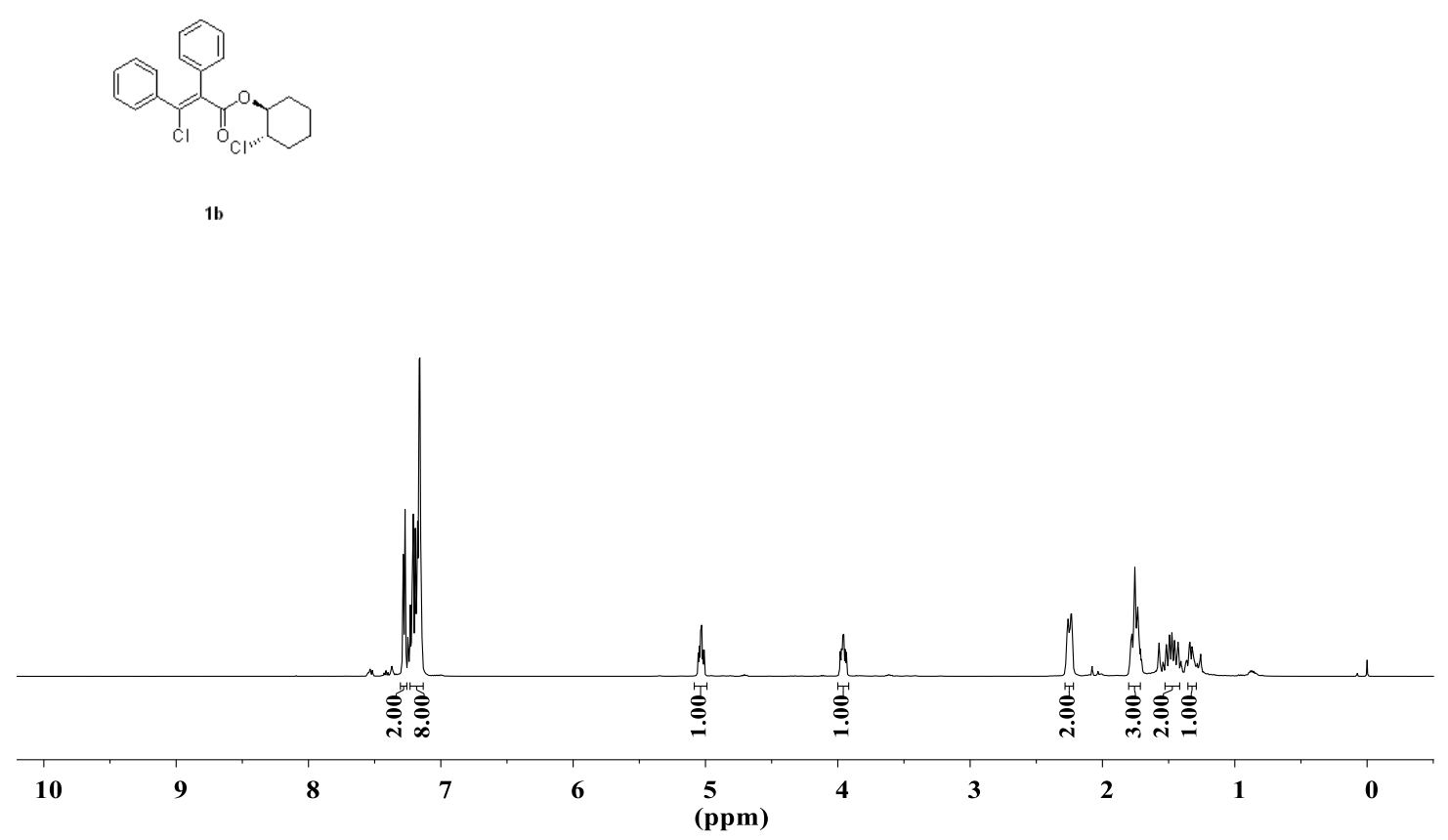

(500 MHz for ${ }^{1} \mathrm{H}$ NMR with $\mathrm{CDCl}_{3}$ as solvent) 

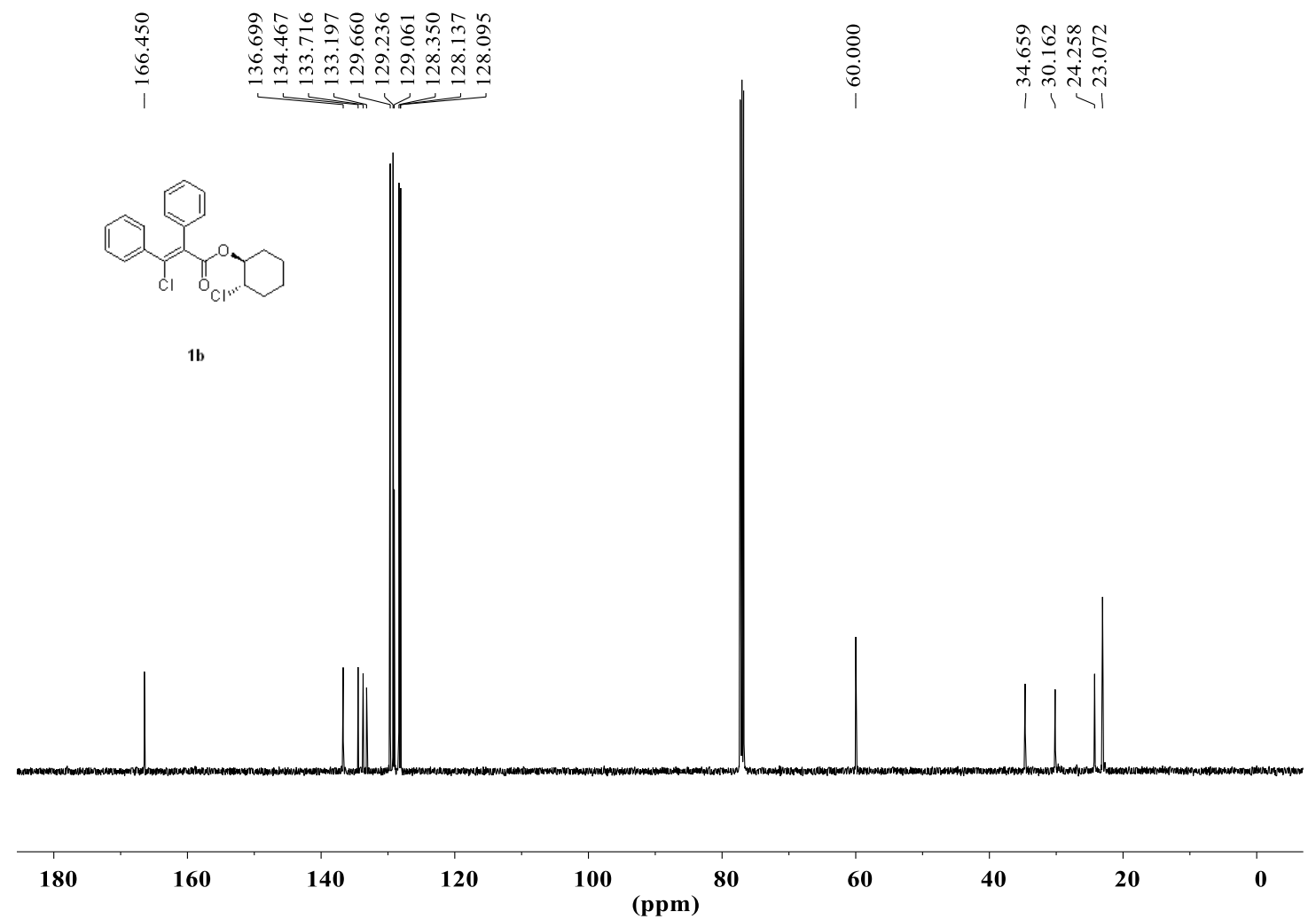

(125 $\mathrm{MHz}$ for ${ }^{13} \mathrm{C} \mathrm{NMR}$ with $\mathrm{CDCl}_{3}$ as solvent)

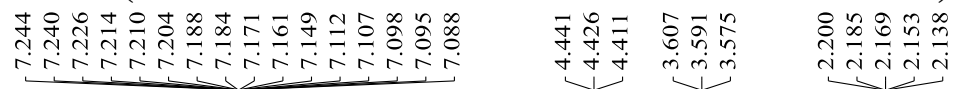<smiles>O=C(O)C(Cl)(c1ccccc1)c1ccccc1</smiles>

2b

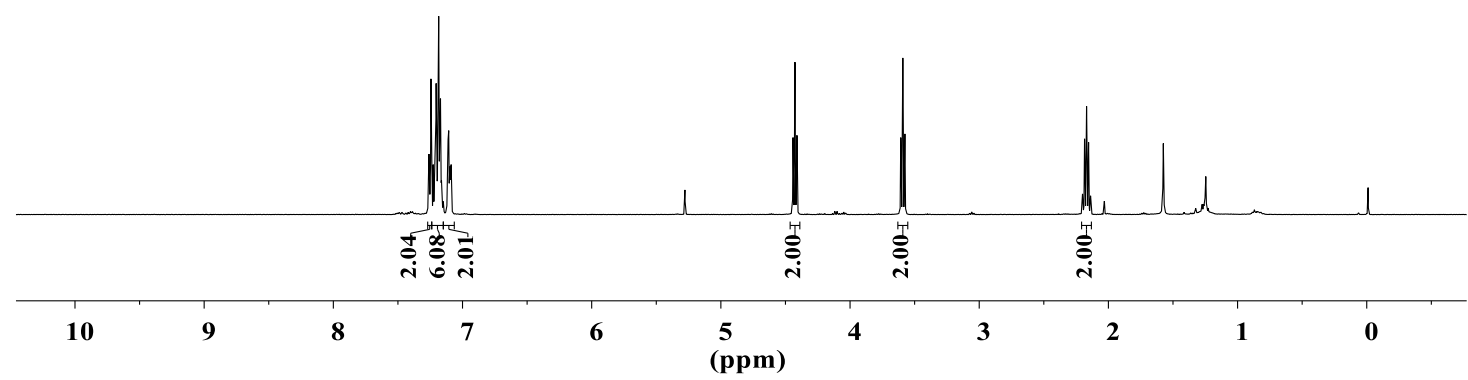

(500 MHz for ${ }^{1} \mathrm{H}$ NMR with $\mathrm{CDCl}_{3}$ as solvent) 


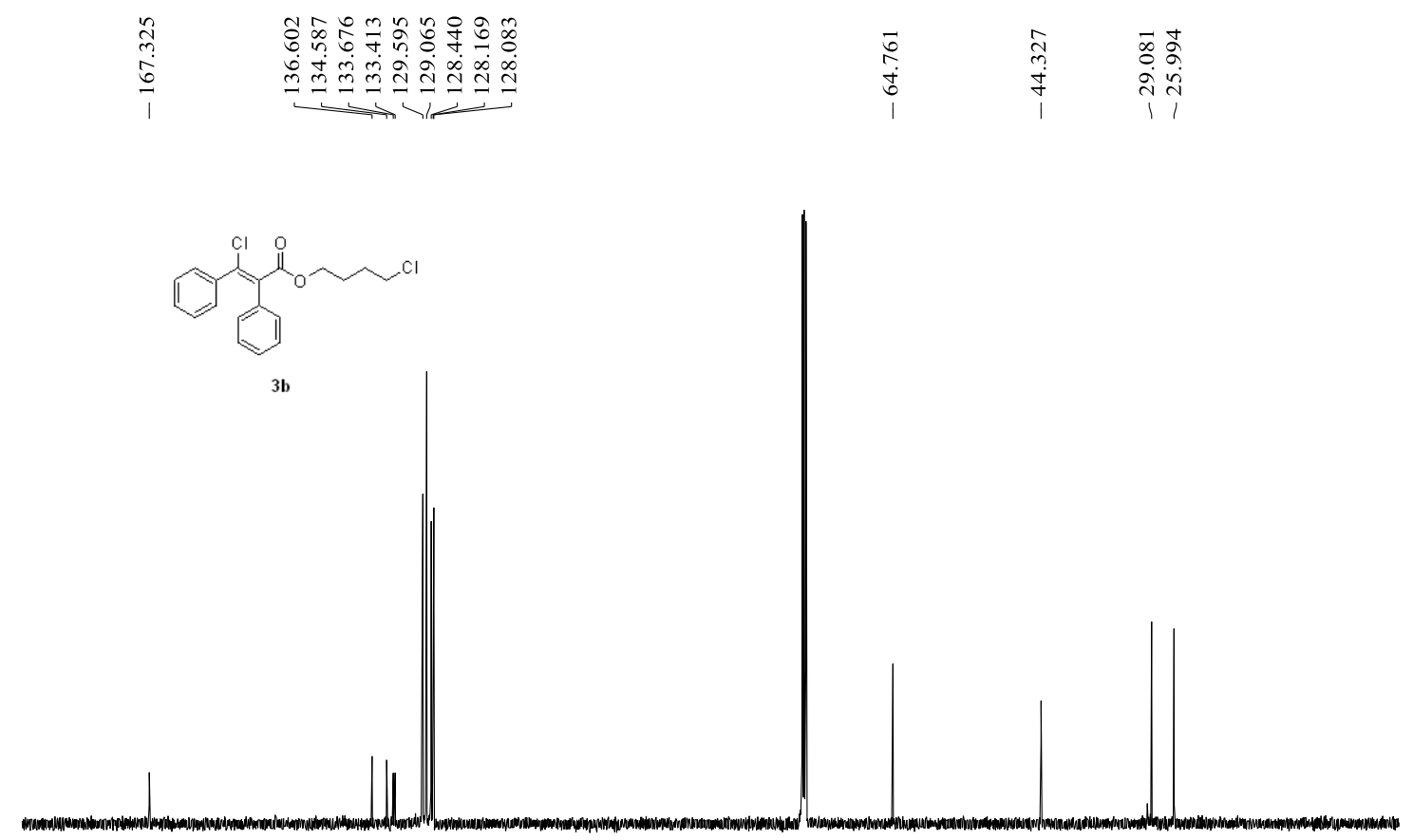

\begin{tabular}{|c|c|c|c|c|c|c|c|c|}
\hline 180 & 160 & 140 & 120 & 100 & 80 & 60 & 40 & 20 \\
\hline
\end{tabular}

(125 MHz for ${ }^{13} \mathrm{C}$ NMR with $\mathrm{CDCl}_{3}$ as solvent)

\begin{tabular}{|c|c|}
\hline 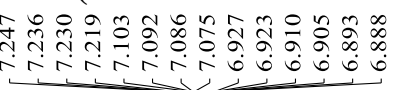 & 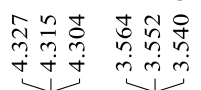 \\
\hline
\end{tabular}

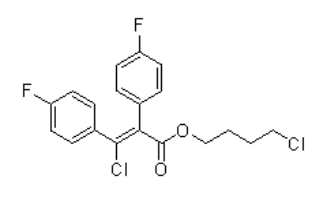

$4 \mathbf{b}$

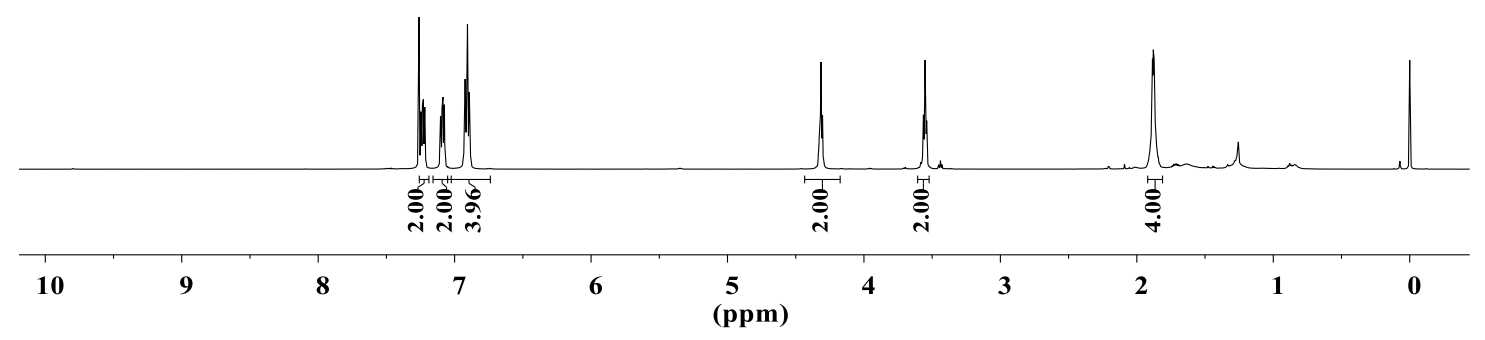

(500 MHz for ${ }^{1} \mathrm{H}$ NMR with $\mathrm{CDCl}_{3}$ as solvent) 


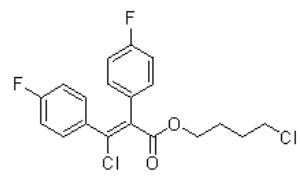

4b

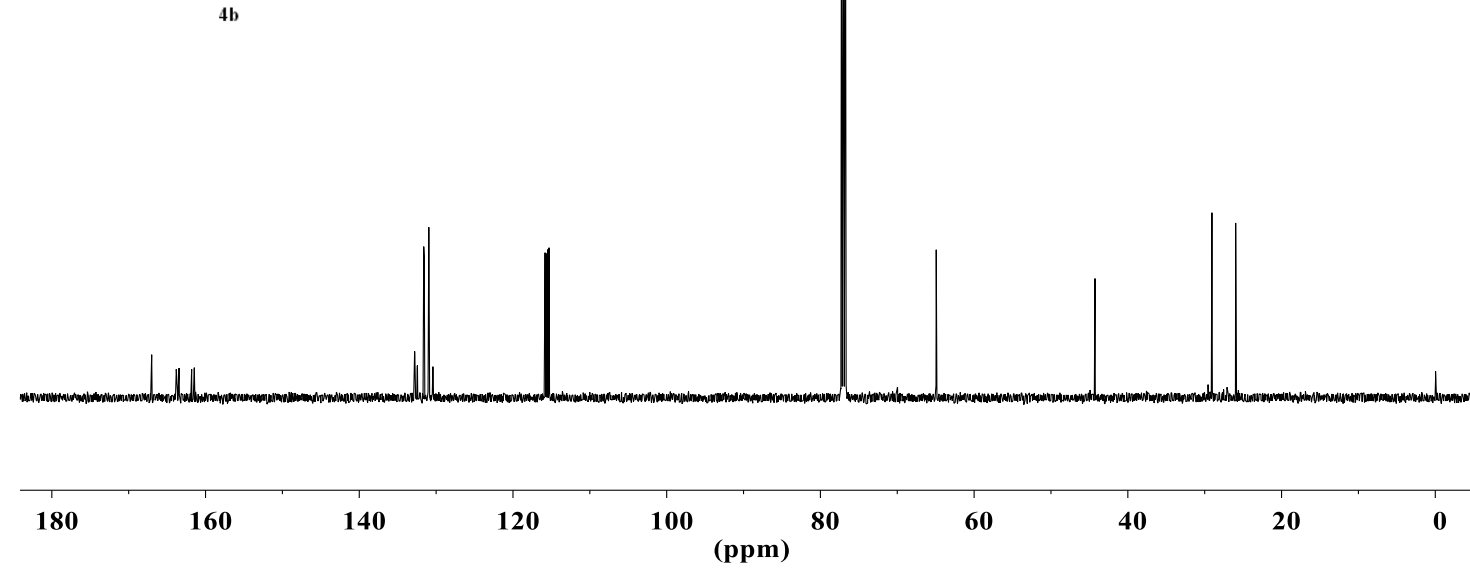

(125 MHz for ${ }^{13} \mathrm{C}$ NMR with $\mathrm{CDCl}_{3}$ as solvent)

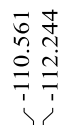

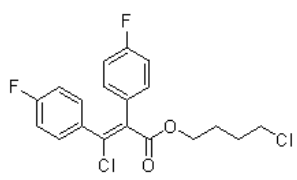

$4 \mathrm{~b}$

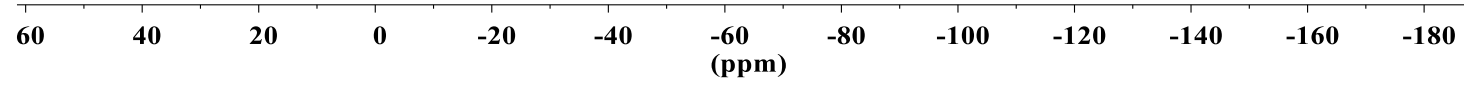

(470 MHz for ${ }^{19} \mathrm{~F}$ NMR with $\mathrm{CDCl}_{3}$ as solvent) 


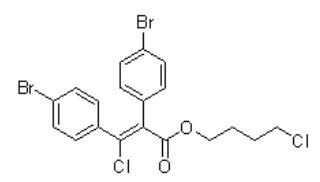

$5 b$

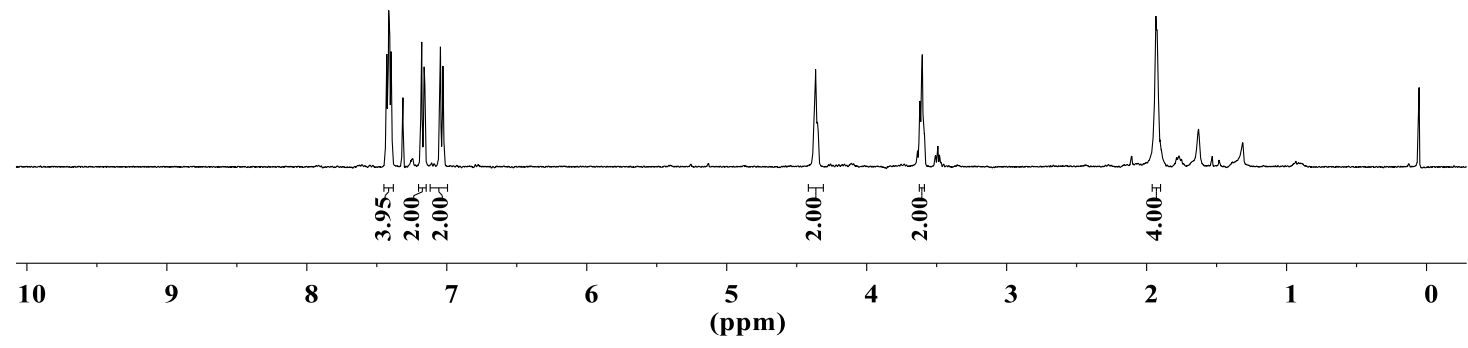

(500 MHz for ${ }^{1} \mathrm{H}$ NMR with $\mathrm{CDCl}_{3}$ as solvent)

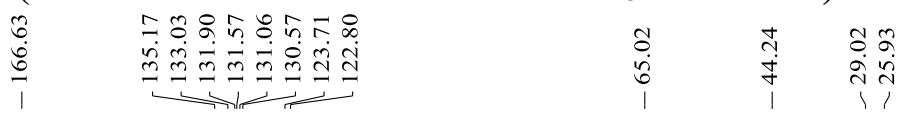

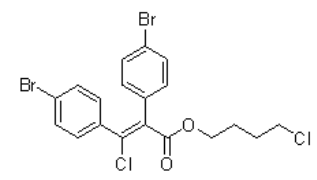

$5 b$

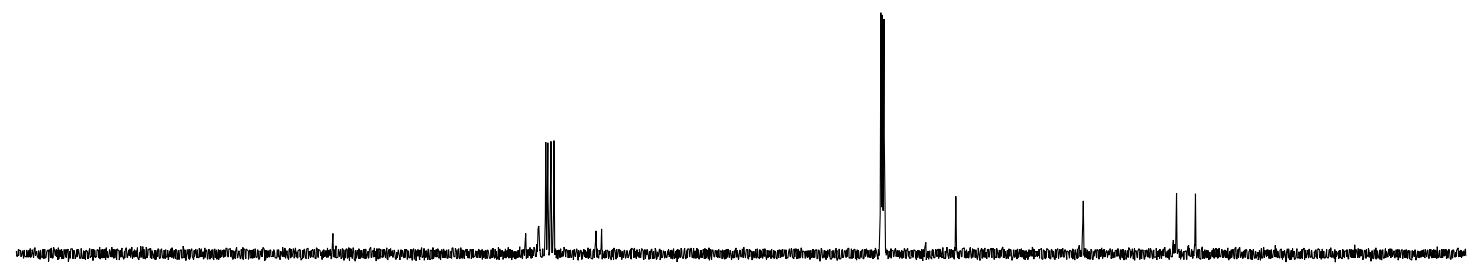

200

180

$160 \quad 140$

100

80

60

40

20

(125 MHz for ${ }^{13} \mathrm{C}$ NMR with $\mathrm{CDCl}_{3}$ as solvent) 


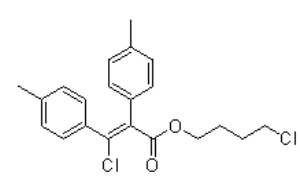

$6 \mathbf{b}$

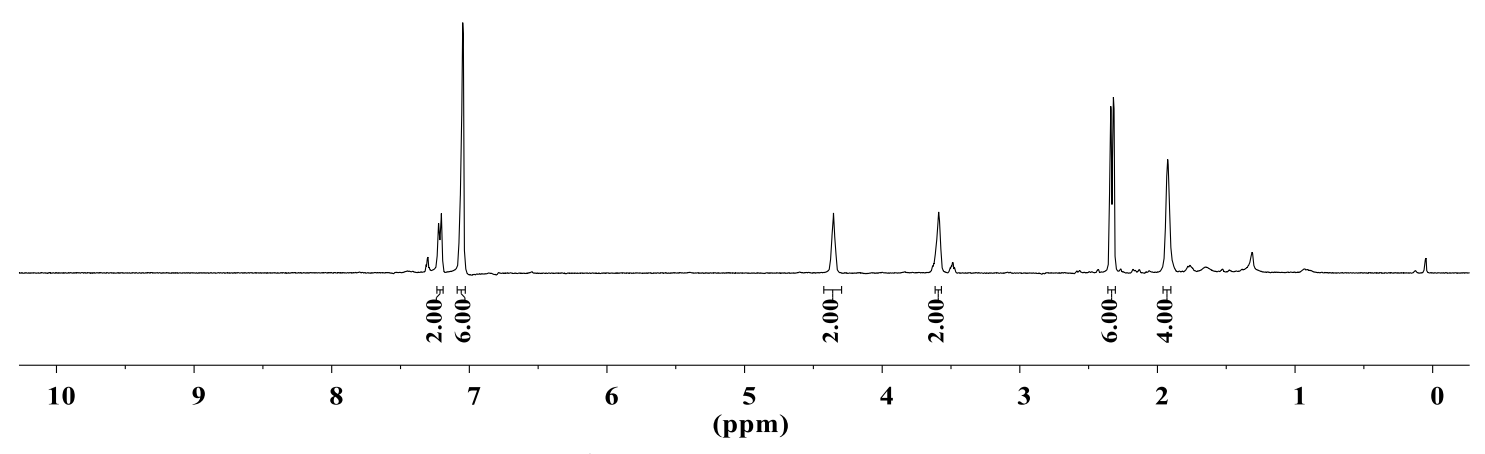

(500 $\mathrm{MHz}$ for ${ }^{1} \mathrm{H}$ NMR with $\mathrm{CDCl}_{3}$ as solvent)

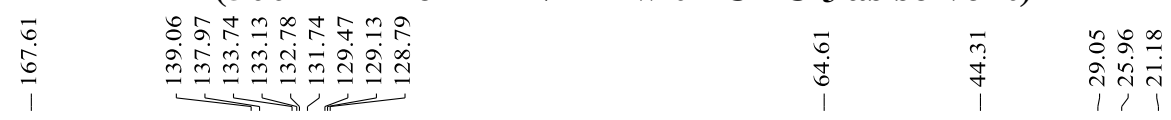

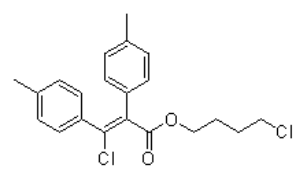

$6 \mathbf{b}$

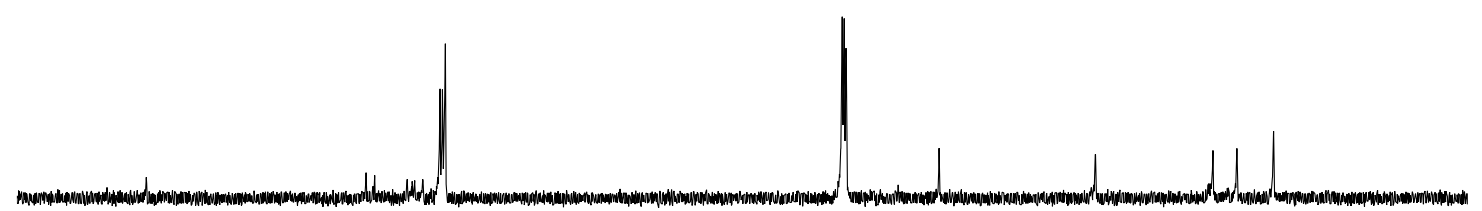

(125 MHz for ${ }^{13} \mathrm{C}$ NMR with $\mathrm{CDCl}_{3}$ as solvent) 


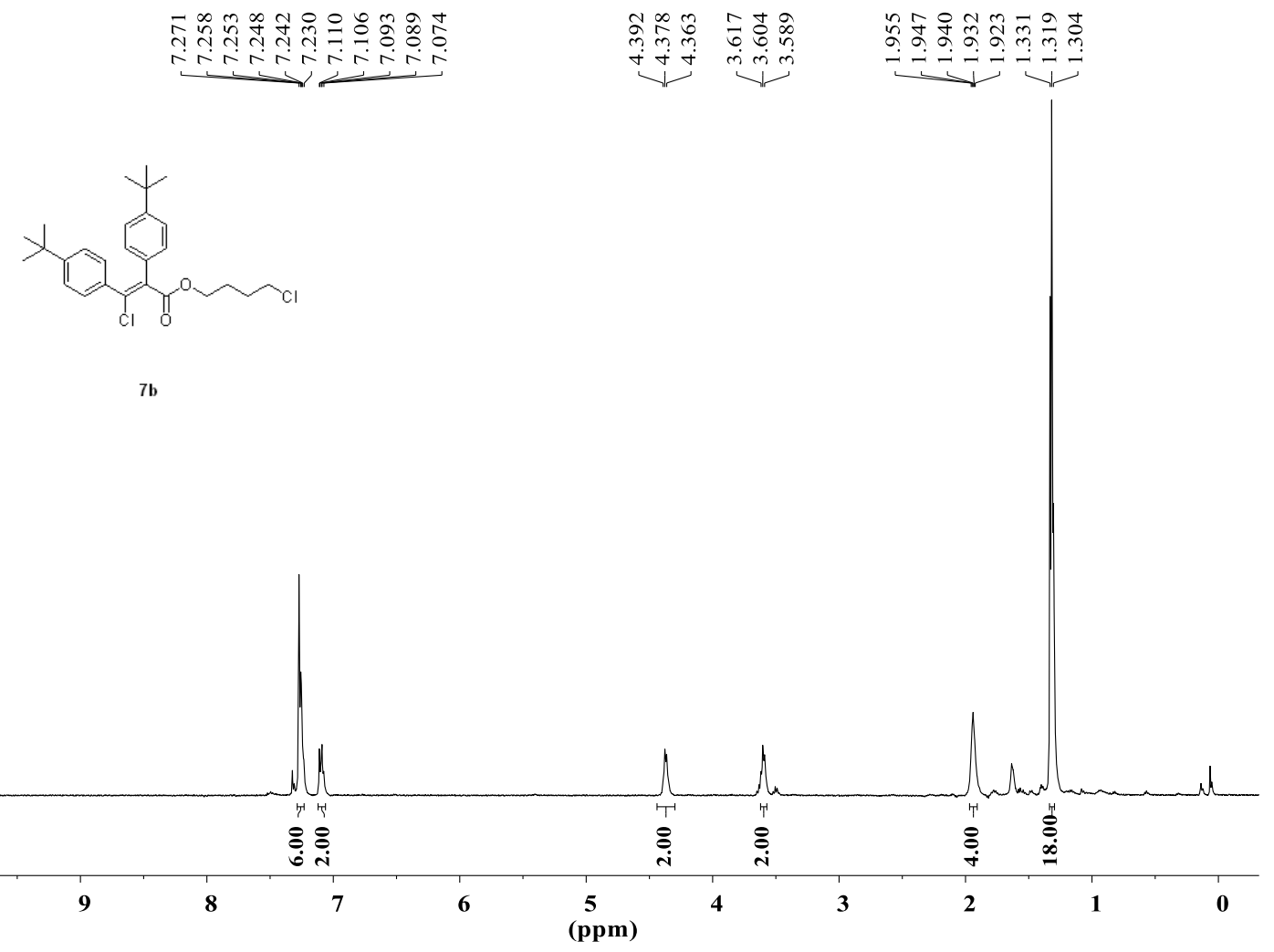

(500 $\mathrm{MHz}$ for ${ }^{1} \mathrm{H}$ NMR with $\mathrm{CDCl}_{3}$ as solvent)
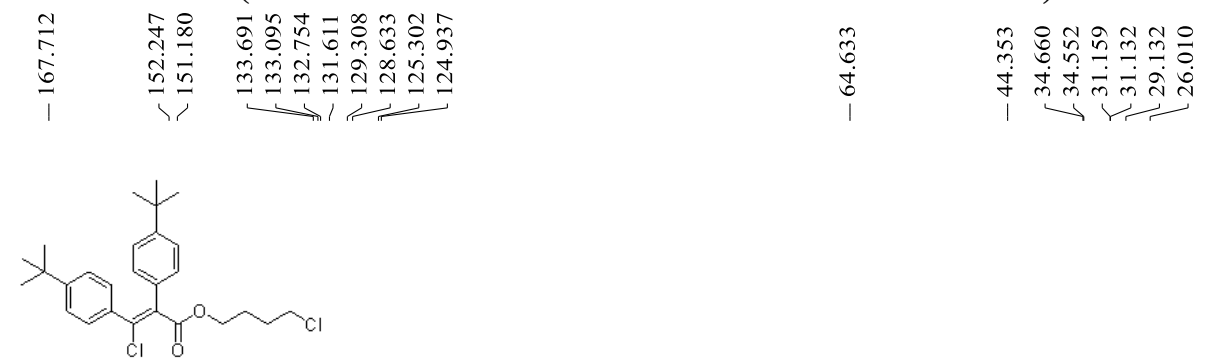

$7 \mathbf{b}$

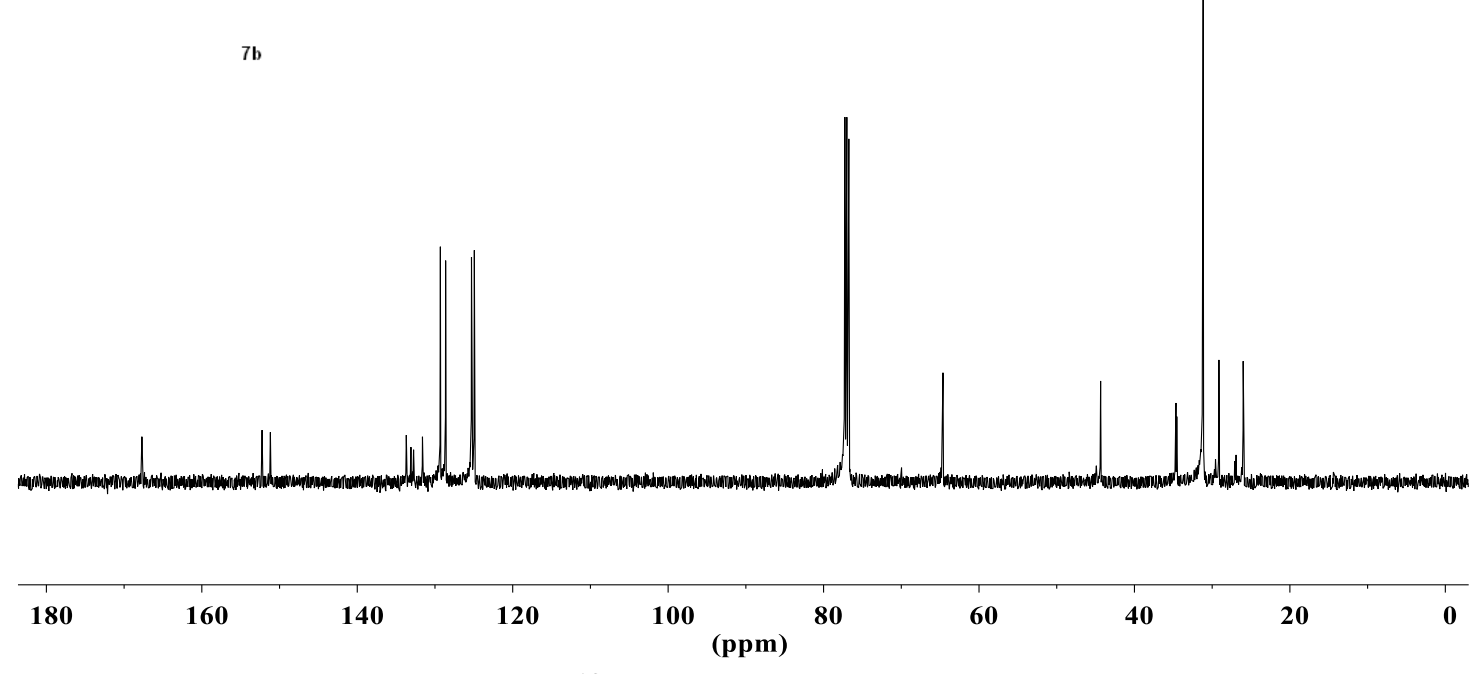

(125 $\mathrm{MHz}$ for ${ }^{13} \mathrm{C} \mathrm{NMR}$ with $\mathrm{CDCl}_{3}$ as solvent) 

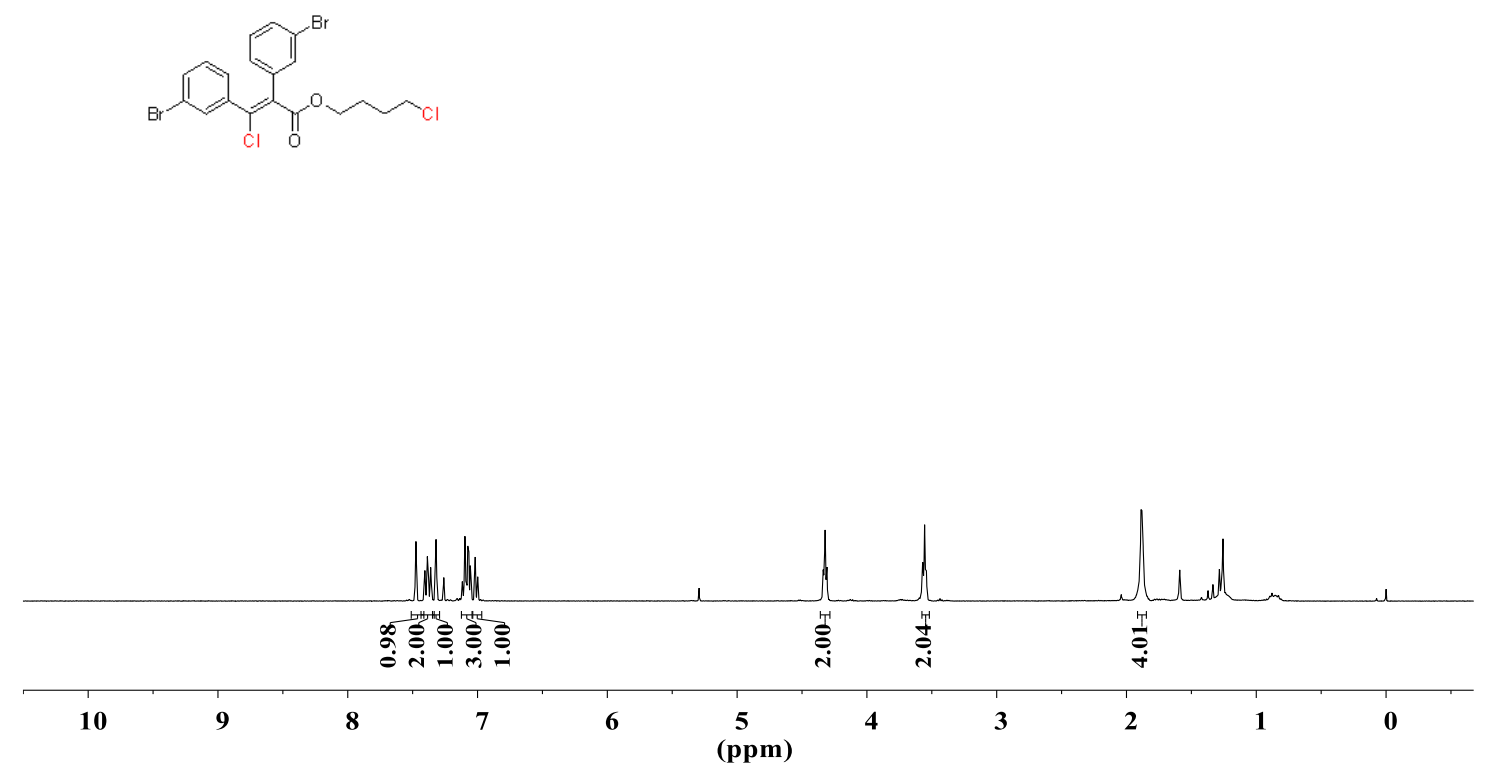

(500 MHz for ${ }^{1} \mathrm{H}$ NMR with $\mathrm{CDCl}_{3}$ as solvent)

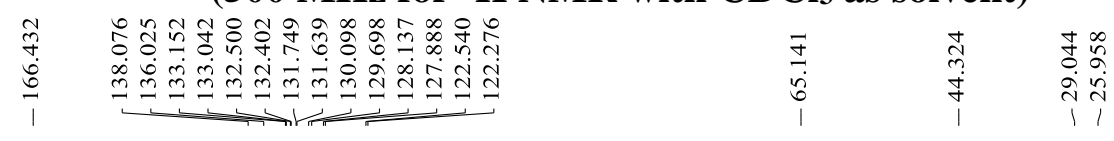

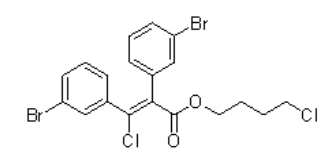

$8 \mathbf{b}$

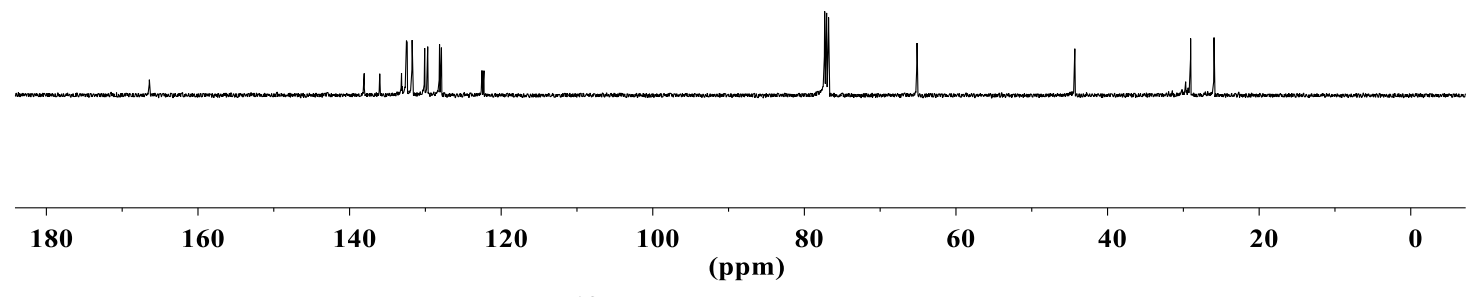

(125 $\mathrm{MHz}$ for ${ }^{13} \mathrm{C} \mathrm{NMR}$ with $\mathrm{CDCl}_{3}$ as solvent) 

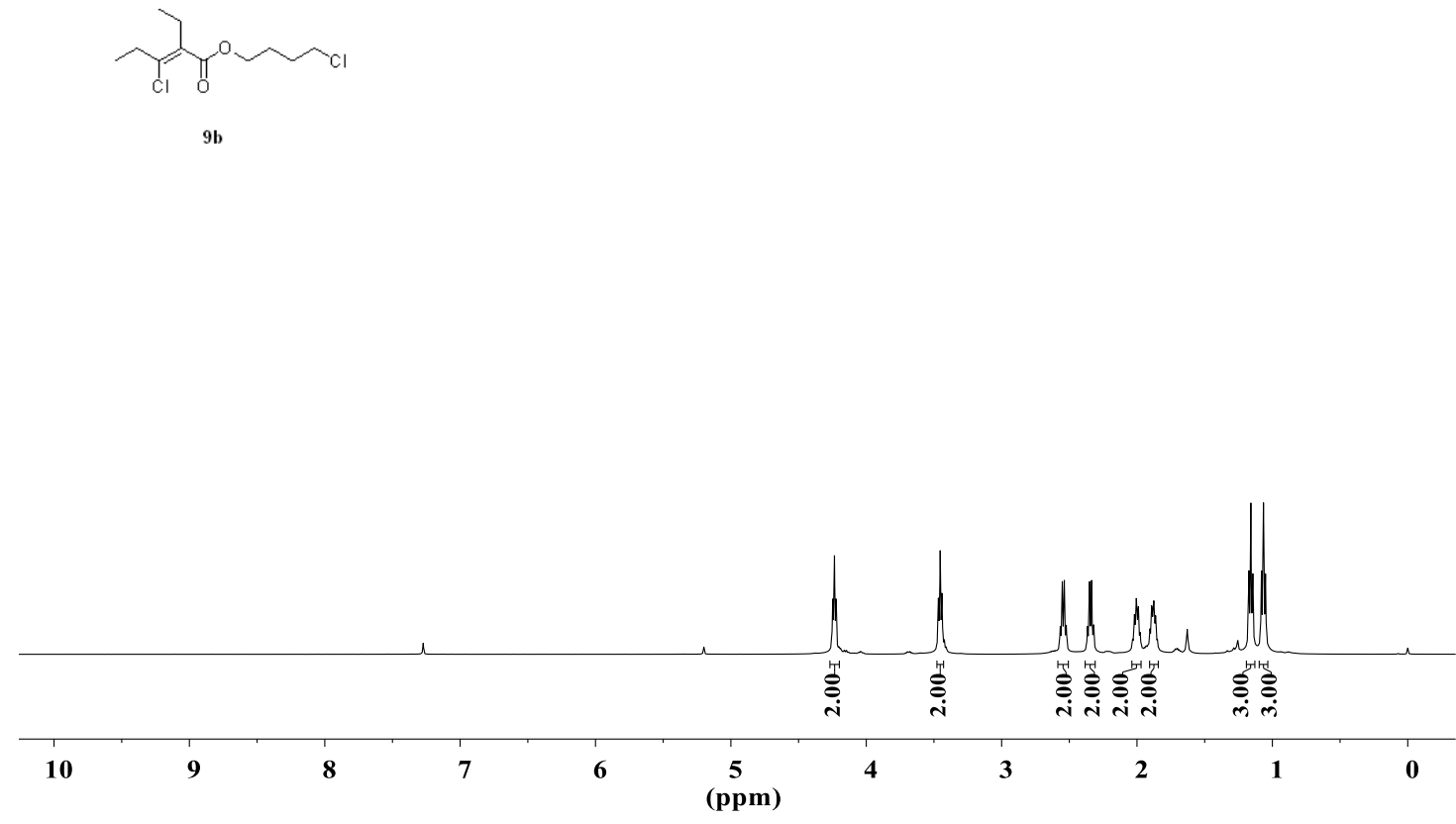

(500 MHz for ${ }^{1} \mathrm{H}$ NMR with $\mathrm{CDCl}_{3}$ as solvent)
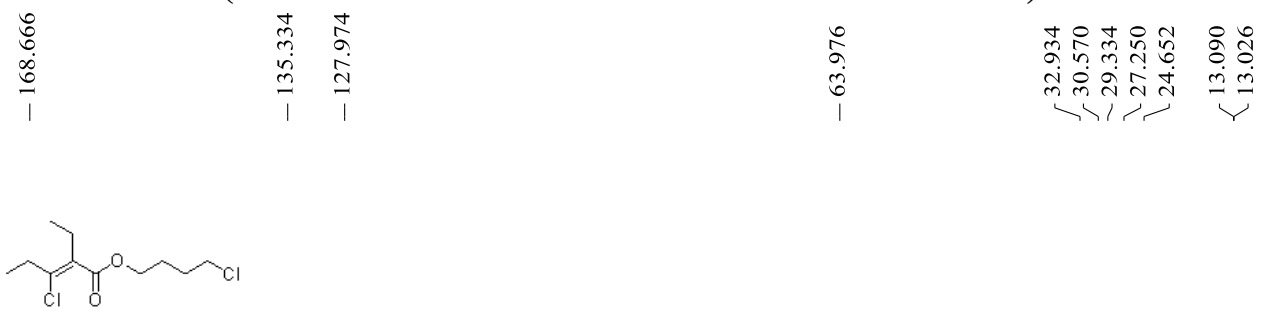

$9 b$

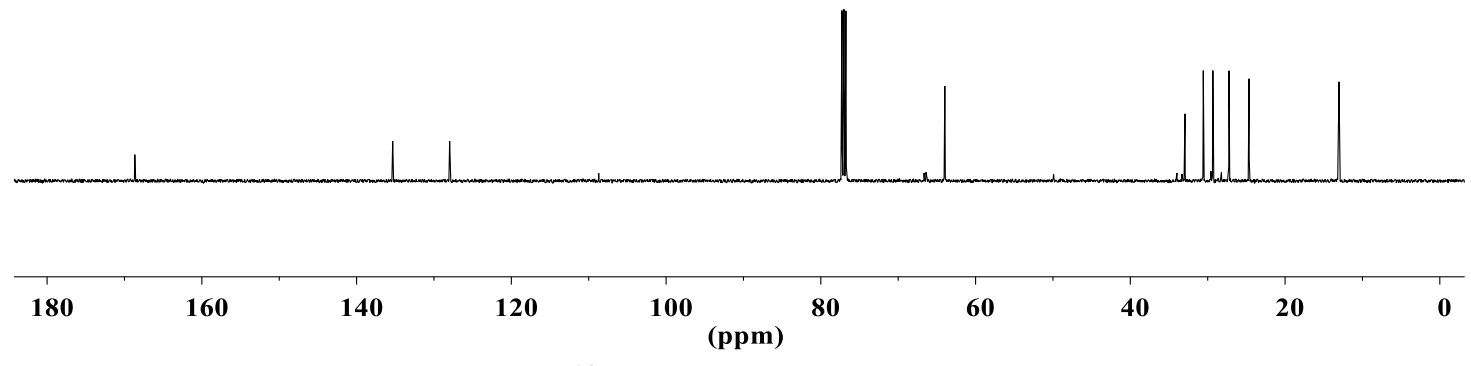

(125 MHz for ${ }^{13} \mathrm{C}$ NMR with $\mathrm{CDCl}_{3}$ as solvent) 


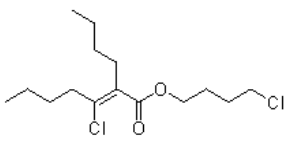

$10 \mathrm{~b}$

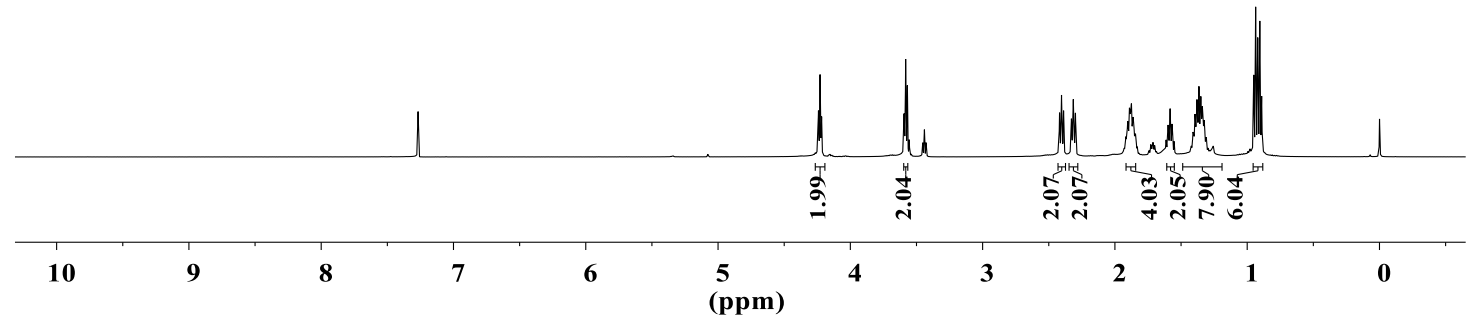

(500 MHz for ${ }^{1} \mathrm{H}$ NMR with $\mathrm{CDCl}_{3}$ as solvent)

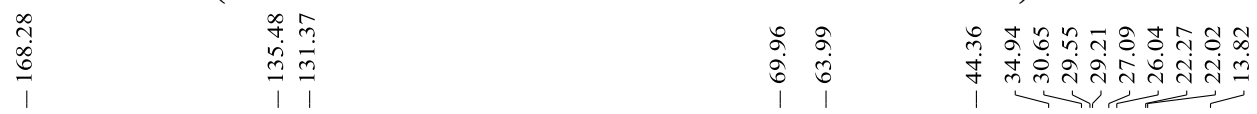

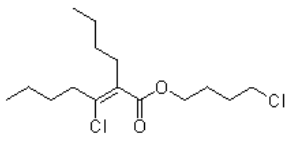

$10 \mathrm{~b}$

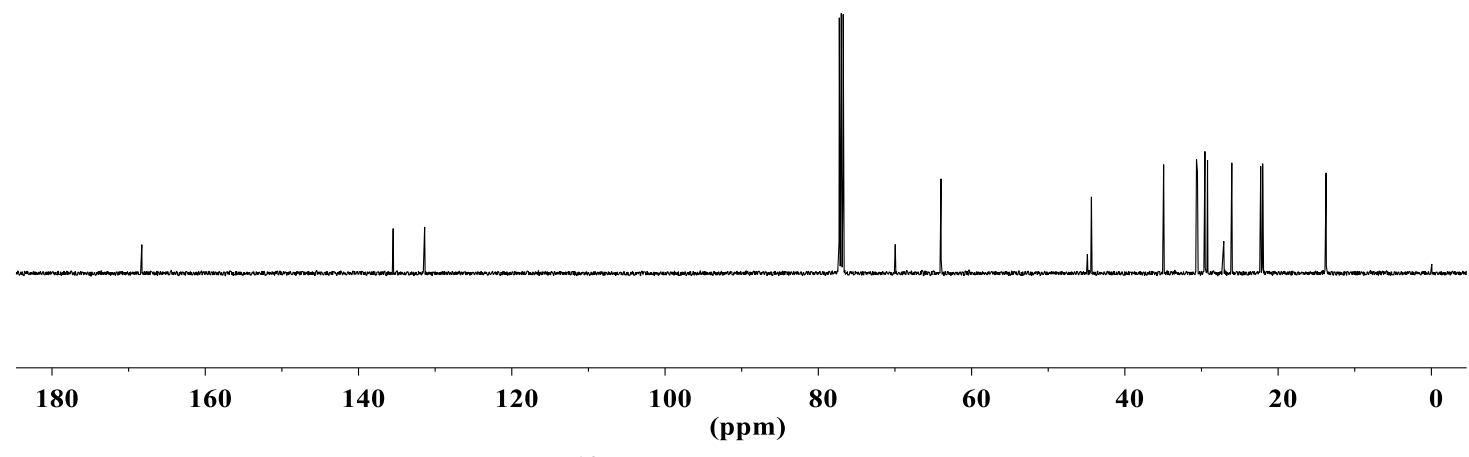

(125 $\mathrm{MHz}$ for ${ }^{13} \mathrm{C} \mathrm{NMR}$ with $\mathrm{CDCl}_{3}$ as solvent) 


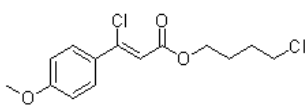

11b

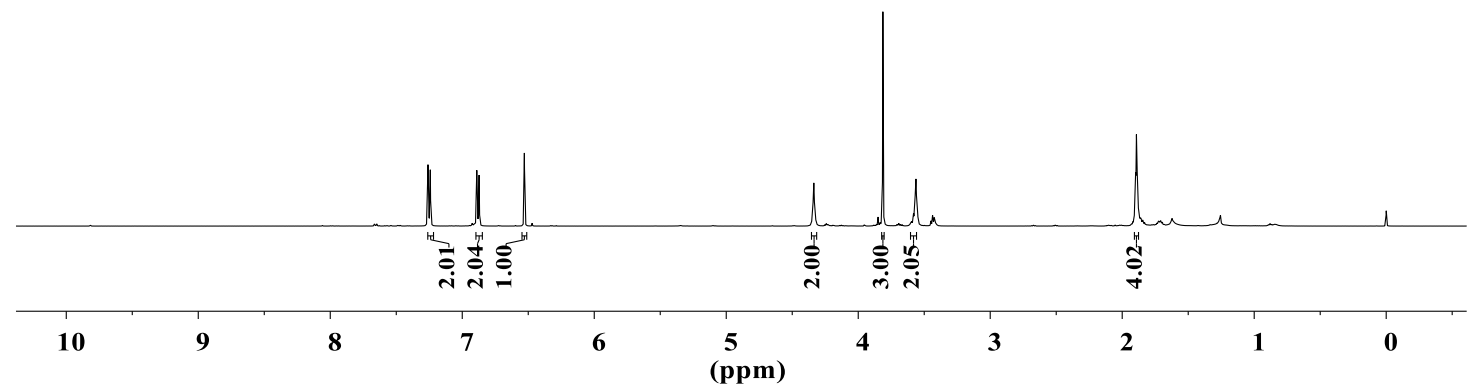

(500 $\mathrm{MHz}$ for ${ }^{1} \mathrm{H}$ NMR with $\mathrm{CDCl}_{3}$ as solvent)

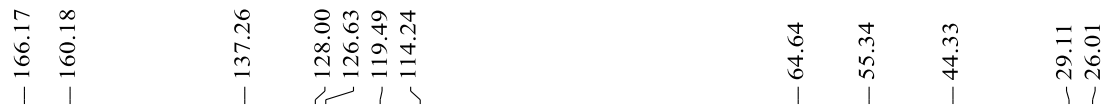

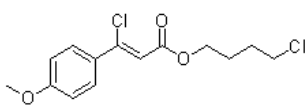

11b

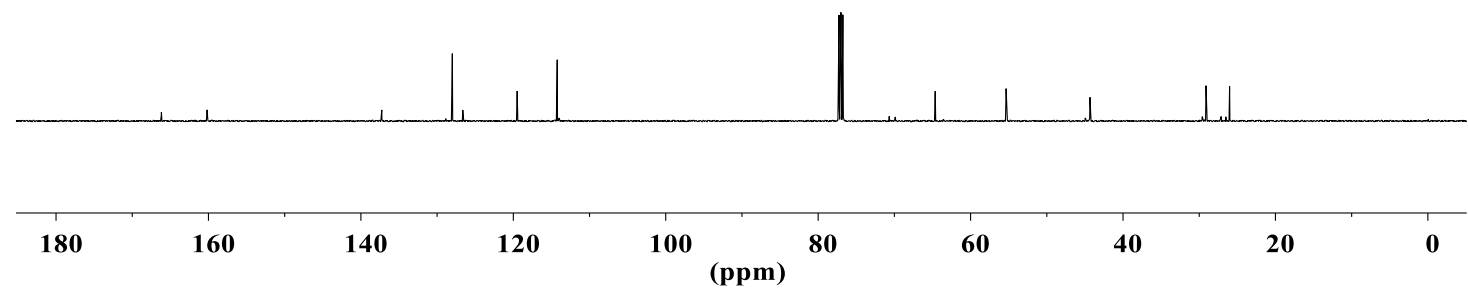

(125 $\mathrm{MHz}$ for ${ }^{13} \mathrm{C}$ NMR with $\mathrm{CDCl}_{3}$ as solvent) 


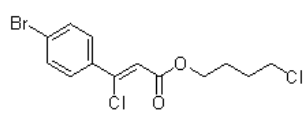

12

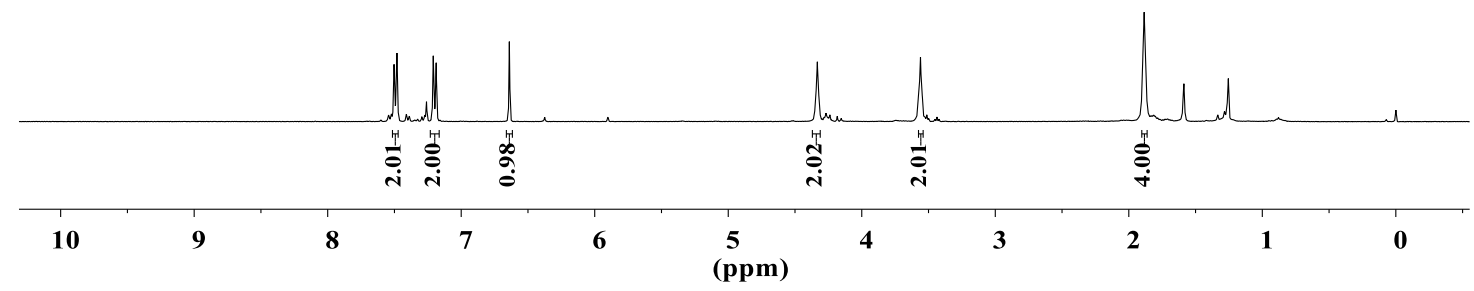

(500 MHz for ${ }^{1} \mathrm{H}$ NMR with $\mathrm{CDCl}_{3}$ as solvent)

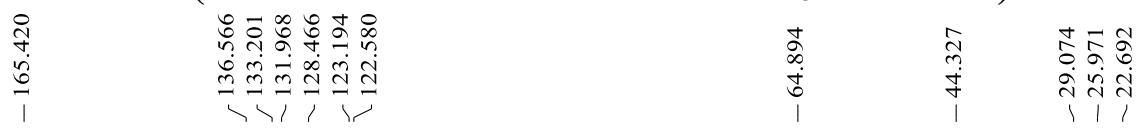

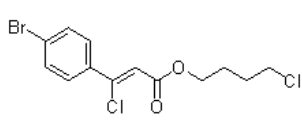

$12 \mathrm{~b}$

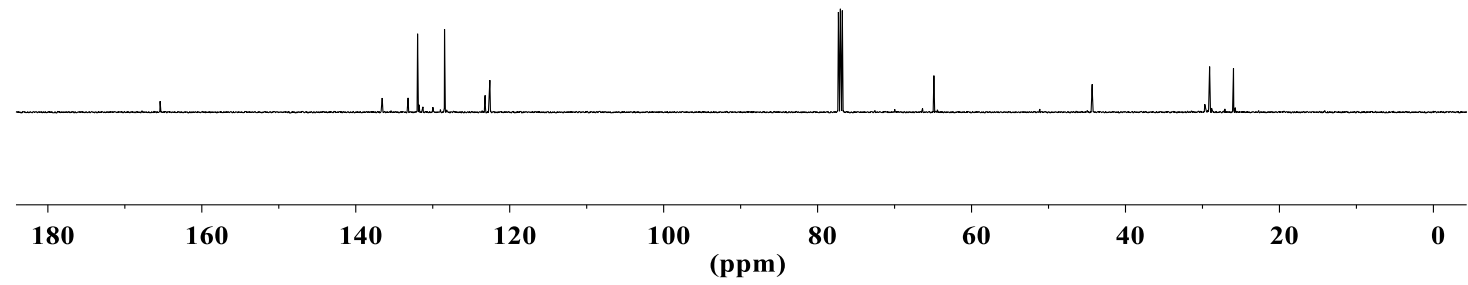

(125 $\mathrm{MHz}$ for ${ }^{13} \mathrm{C}$ NMR with $\mathrm{CDCl}_{3}$ as solvent) 


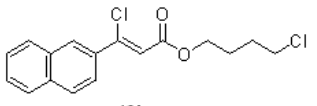

13

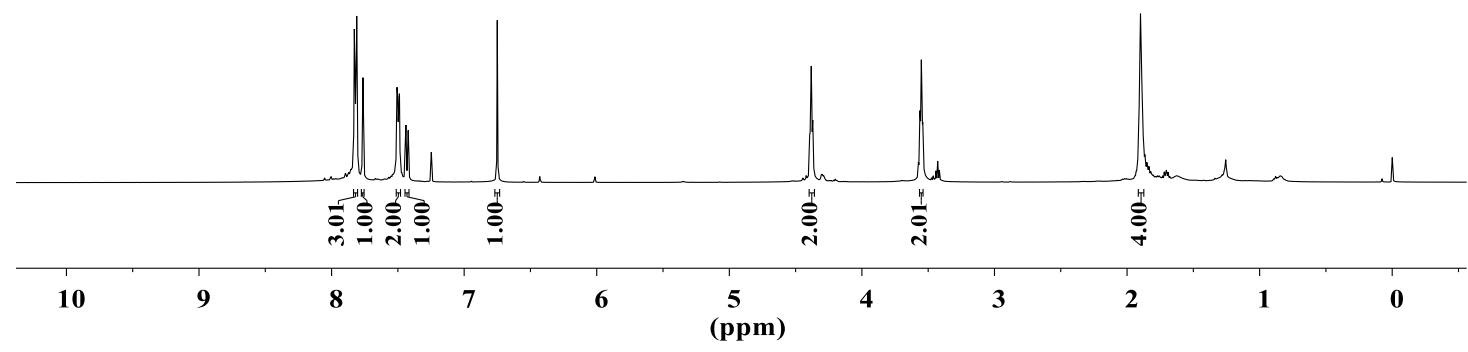

(500 MHz for ${ }^{1} \mathrm{H}$ NMR with $\mathrm{CDCl}_{3}$ as solvent)

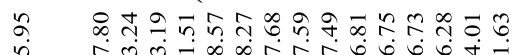

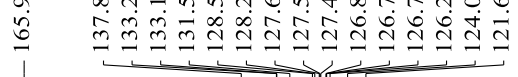

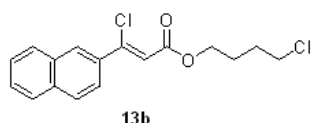

13b

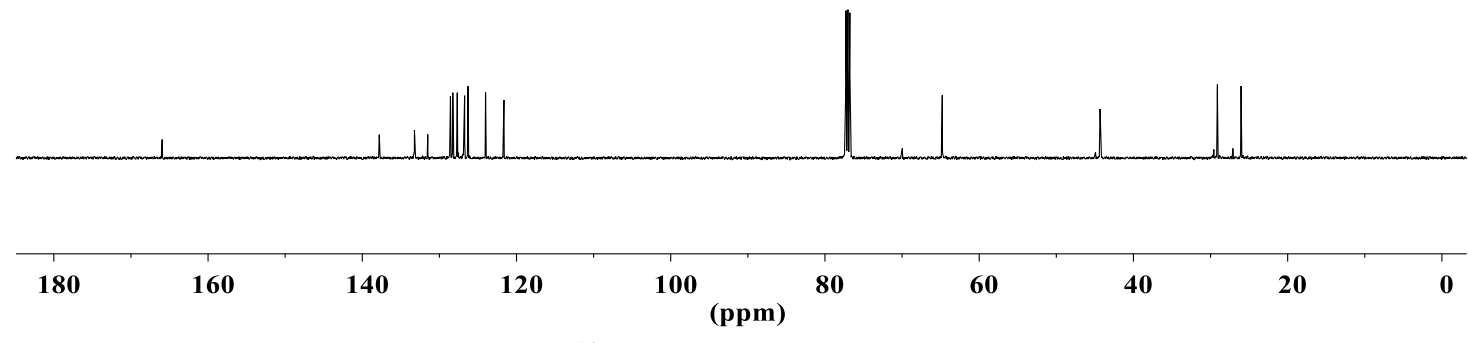

(125 $\mathrm{MHz}$ for ${ }^{13} \mathrm{C} \mathrm{NMR}$ with $\mathrm{CDCl}_{3}$ as solvent) 


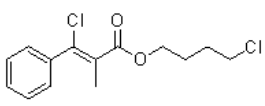

14b

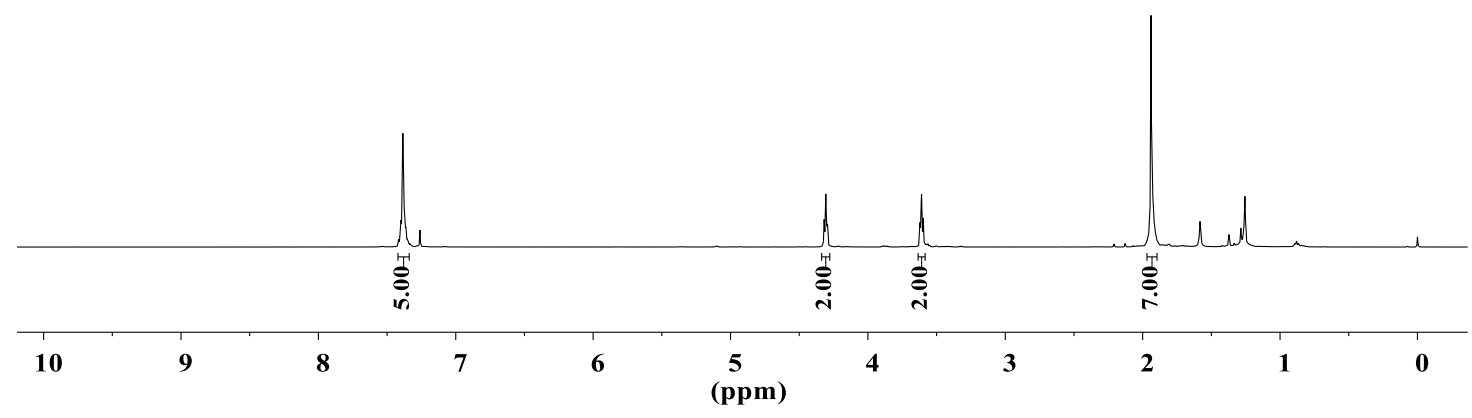

(500 MHz for ${ }^{1} \mathrm{H}$ NMR with $\mathrm{CDCl}_{3}$ as solvent)

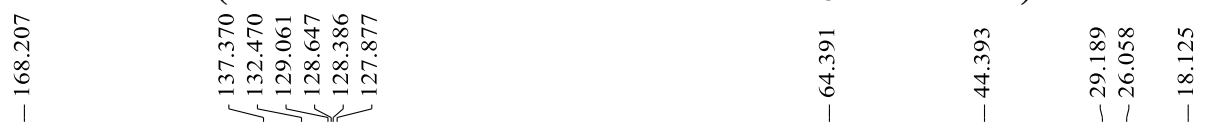

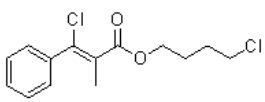

14b

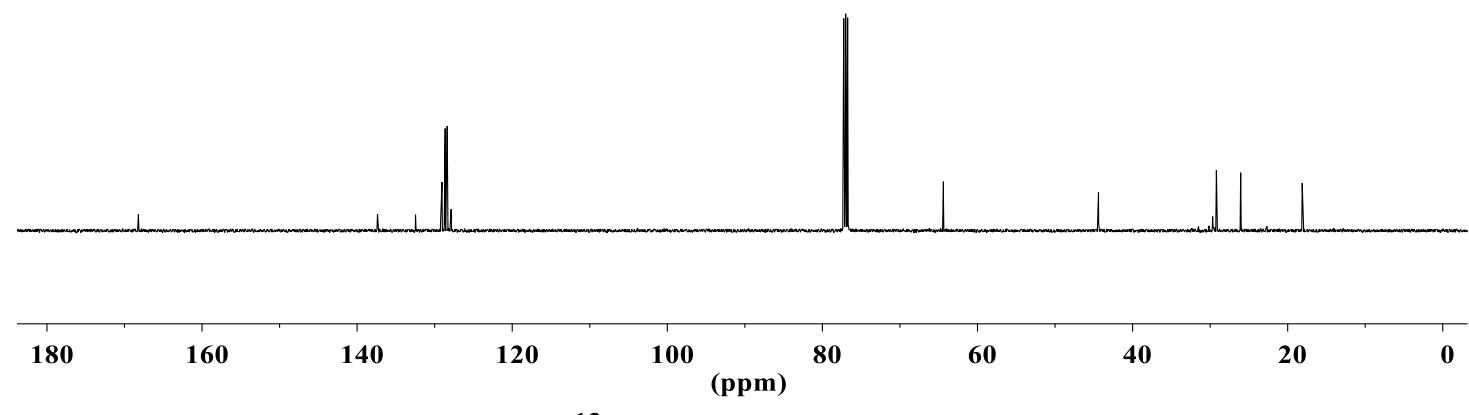

(125 MHz for ${ }^{13} \mathrm{C}$ NMR with $\mathrm{CDCl}_{3}$ as solvent) 


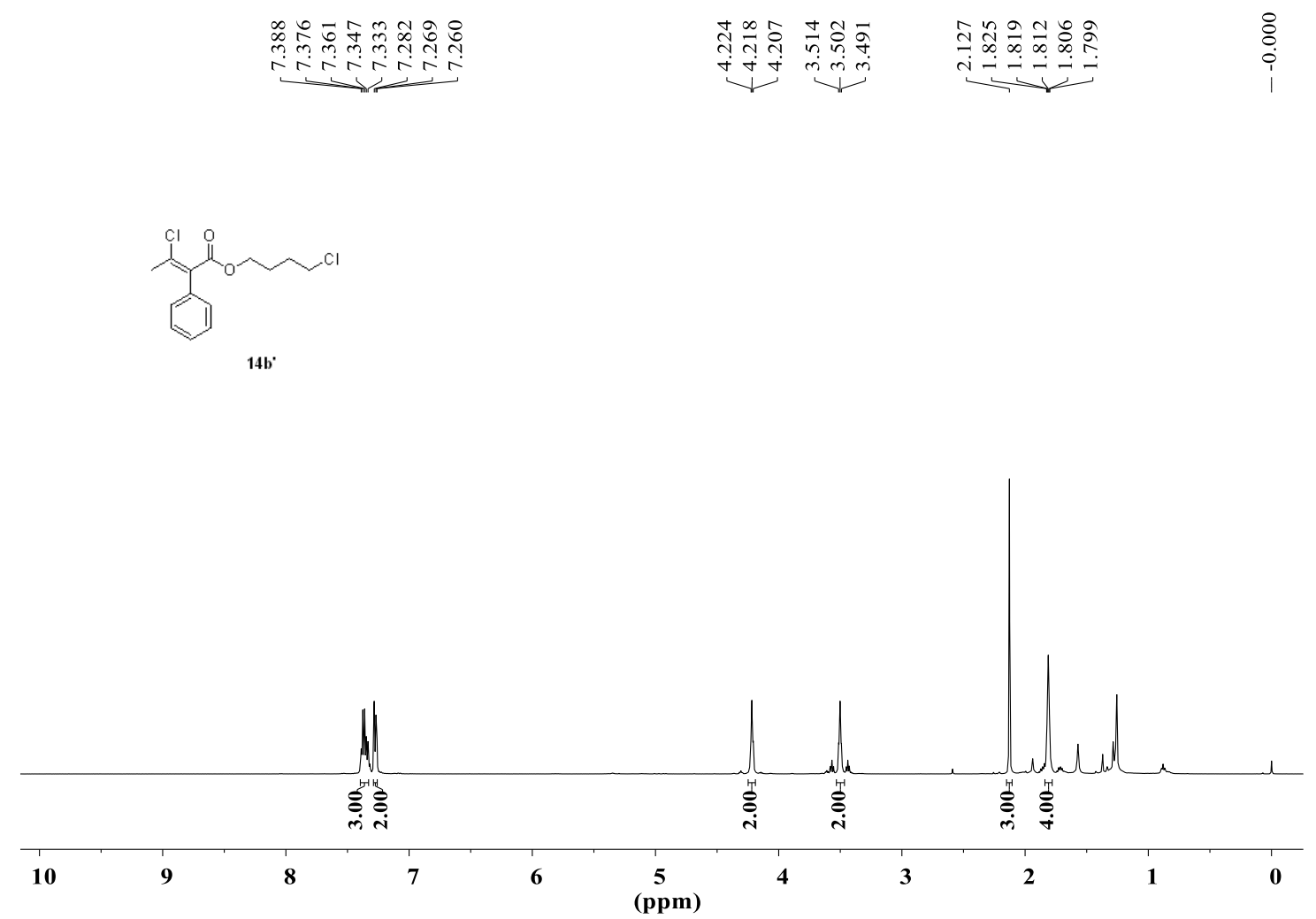

(500 $\mathrm{MHz}$ for ${ }^{1} \mathrm{H}$ NMR with $\mathrm{CDCl}_{3}$ as solvent)

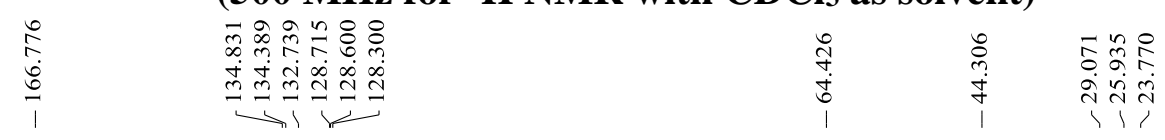
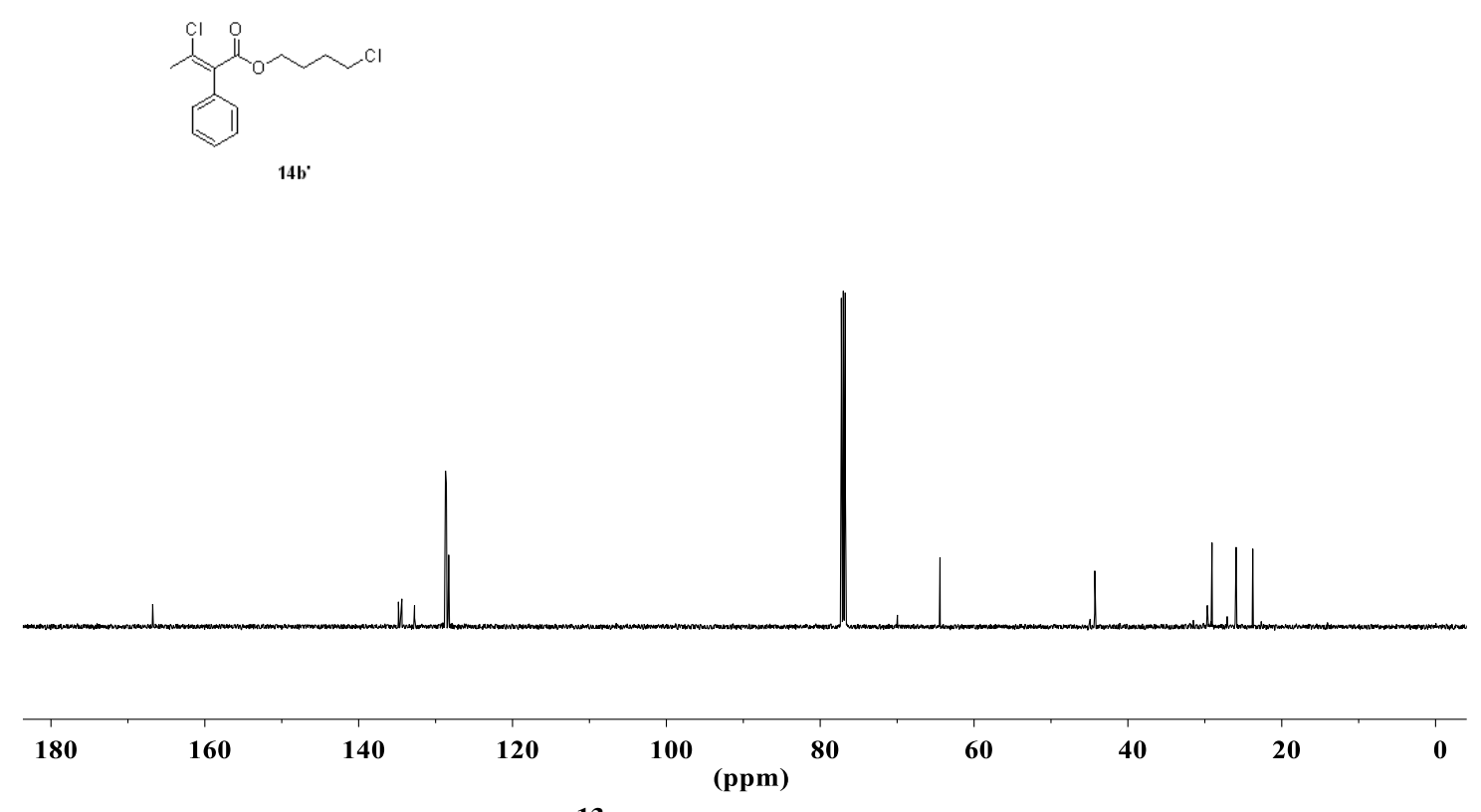

(125 $\mathrm{MHz}$ for ${ }^{13} \mathrm{C} \mathrm{NMR}$ with $\mathrm{CDCl}_{3}$ as solvent) 


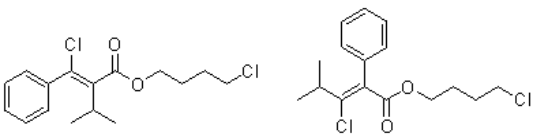

$15 \mathrm{~b}$ and $15 \mathrm{~b}$

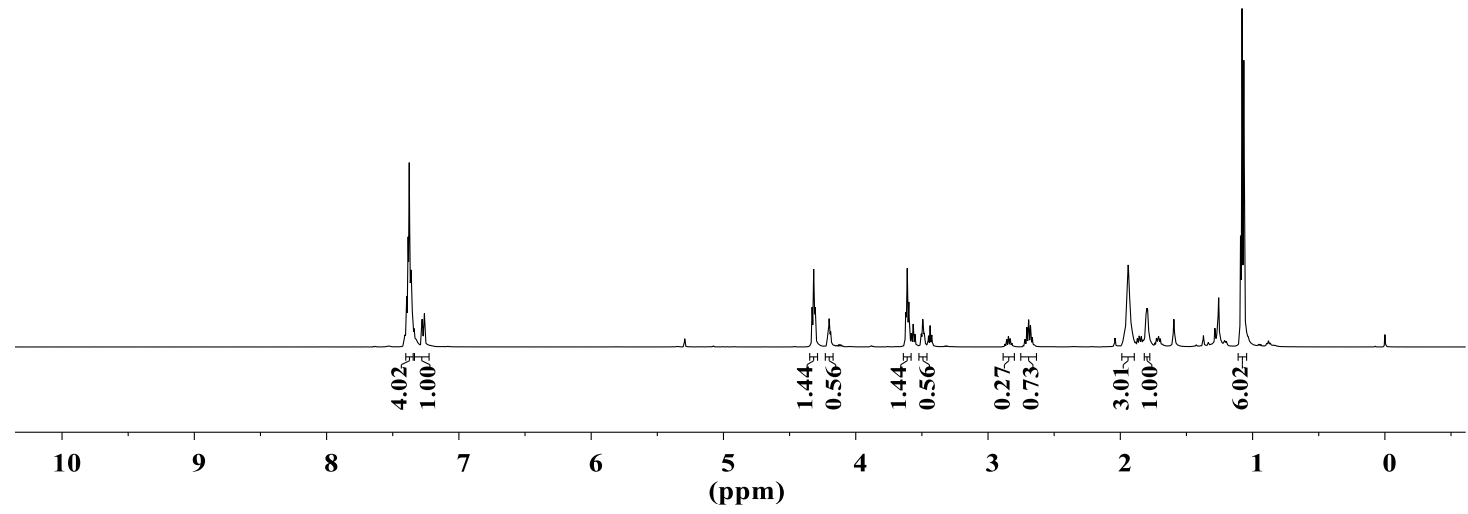

(500 MHz for ${ }^{1} \mathrm{H}$ NMR with $\mathrm{CDCl}_{3}$ as solvent)

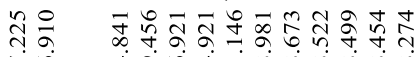

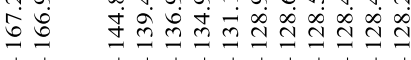

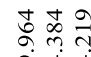

จ่

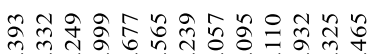

Ұ अ

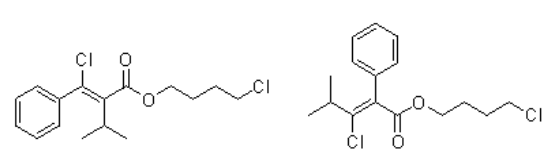

$15 \mathrm{~b}$ and $15 \mathrm{~b}$

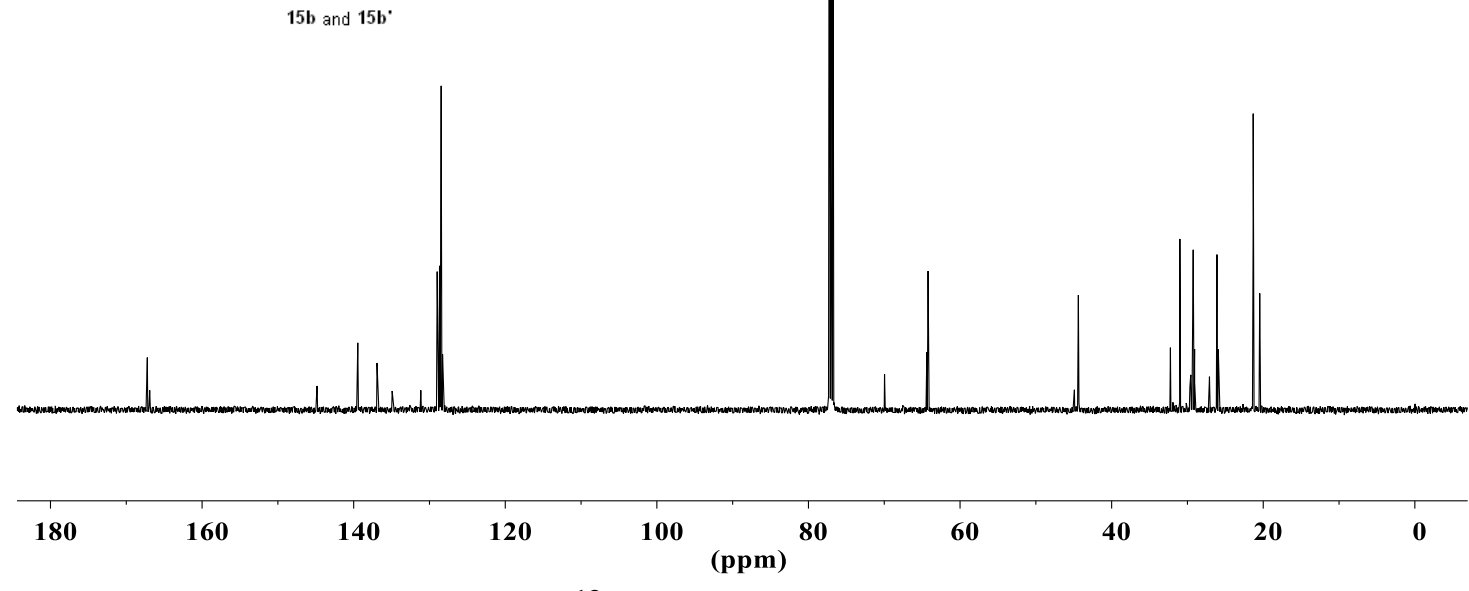

(125 $\mathrm{MHz}$ for ${ }^{13} \mathrm{C} \mathrm{NMR}$ with $\mathrm{CDCl}_{3}$ as solvent) 


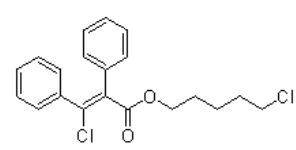

16b

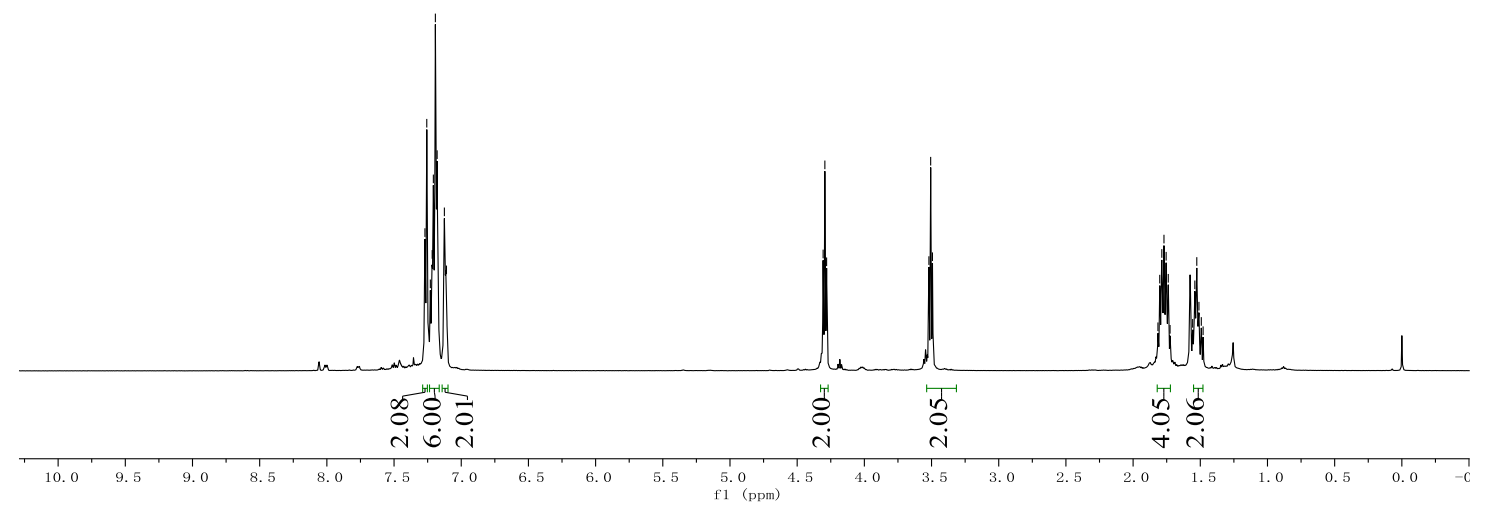

(500 MHz for ${ }^{1} \mathrm{H}$ NMR with $\mathrm{CDCl}_{3}$ as solvent)

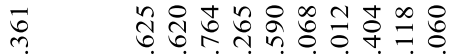

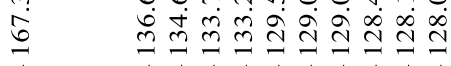

1 (1)

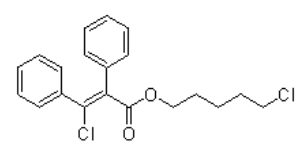

$16 b$
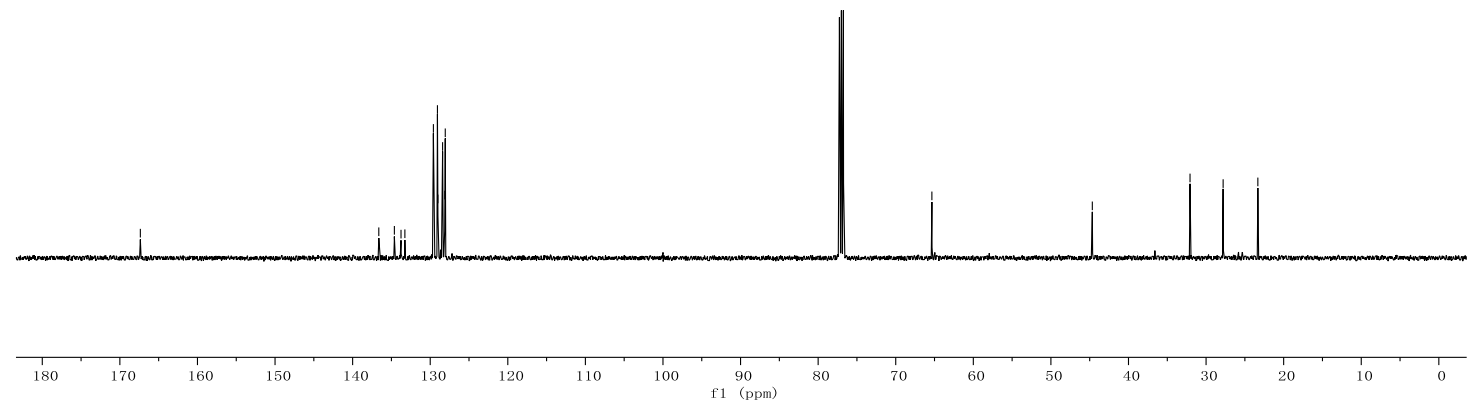

(125 $\mathrm{MHz}$ for ${ }^{13} \mathrm{C} \mathrm{NMR}$ with $\mathrm{CDCl}_{3}$ as solvent) 


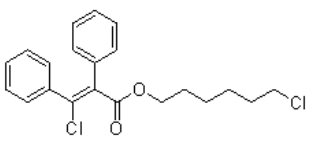

171

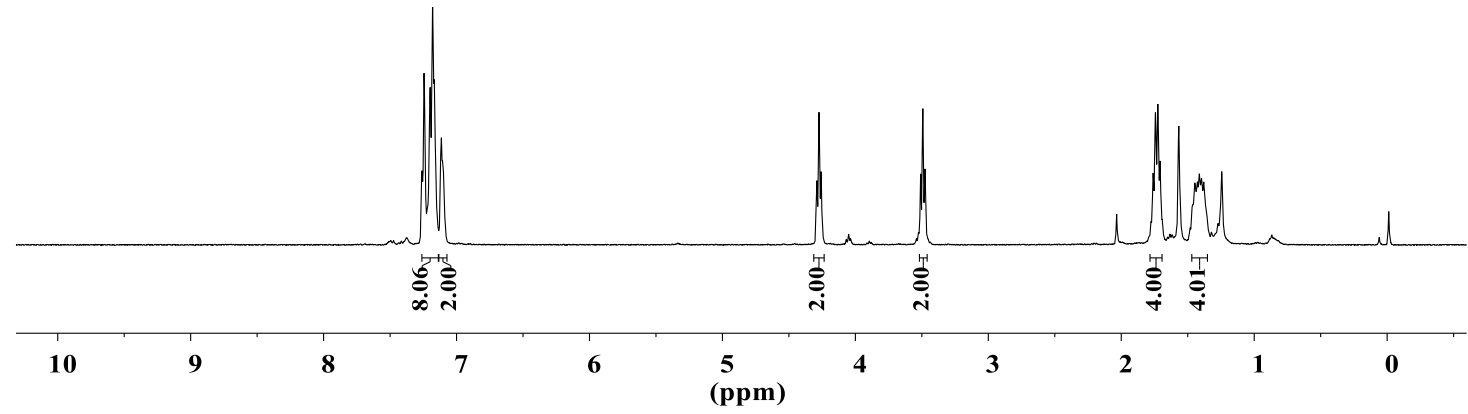

(500 MHz for ${ }^{1} \mathrm{H}$ NMR with $\mathrm{CDCl}_{3}$ as solvent)

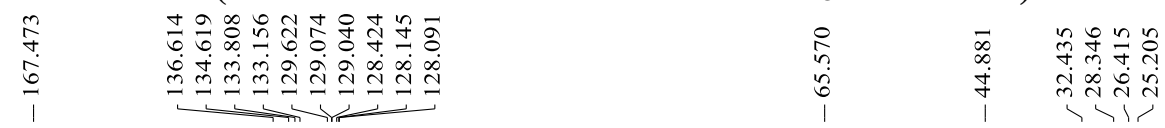
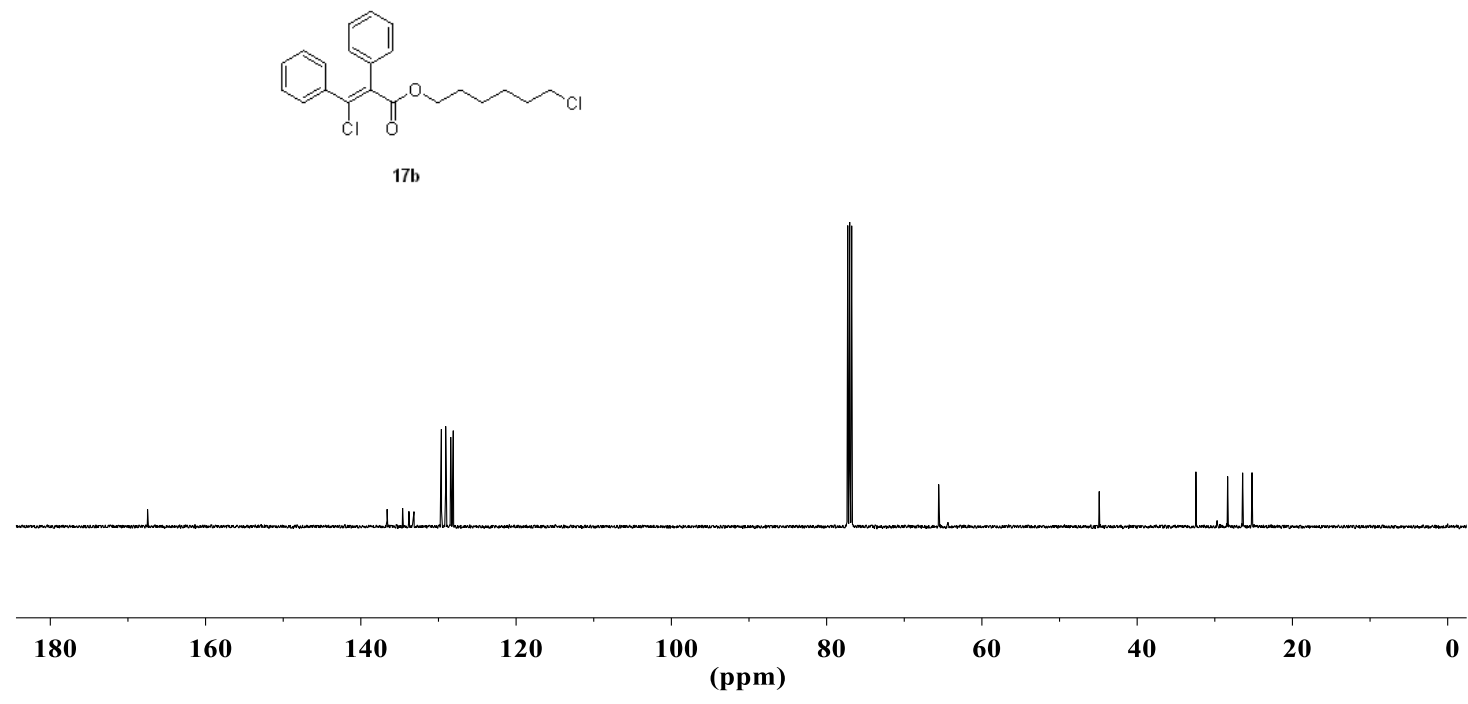

(125 $\mathrm{MHz}$ for ${ }^{13} \mathrm{C} \mathrm{NMR}$ with $\mathrm{CDCl}_{3}$ as solvent) 


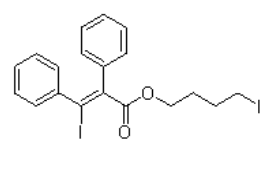

$1 c$

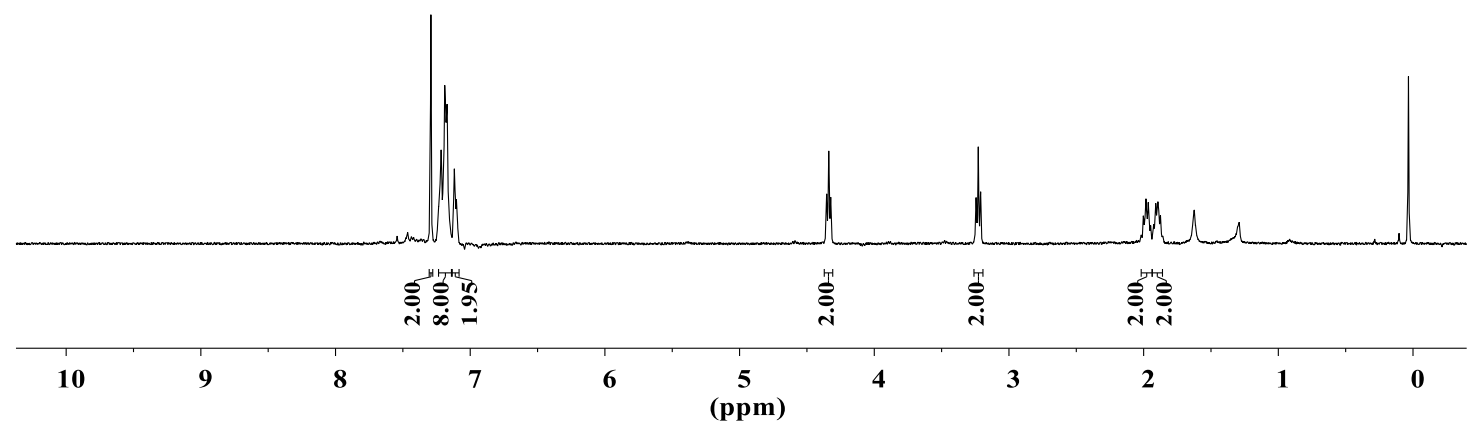

(500 MHz for ${ }^{1} \mathrm{H}$ NMR with $\mathrm{CDCl}_{3}$ as solvent)

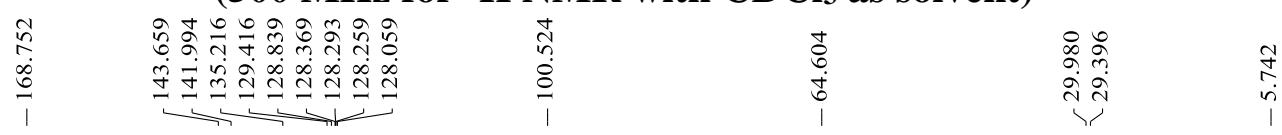

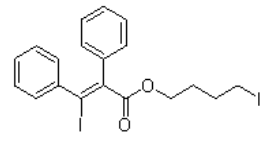

$1 c$

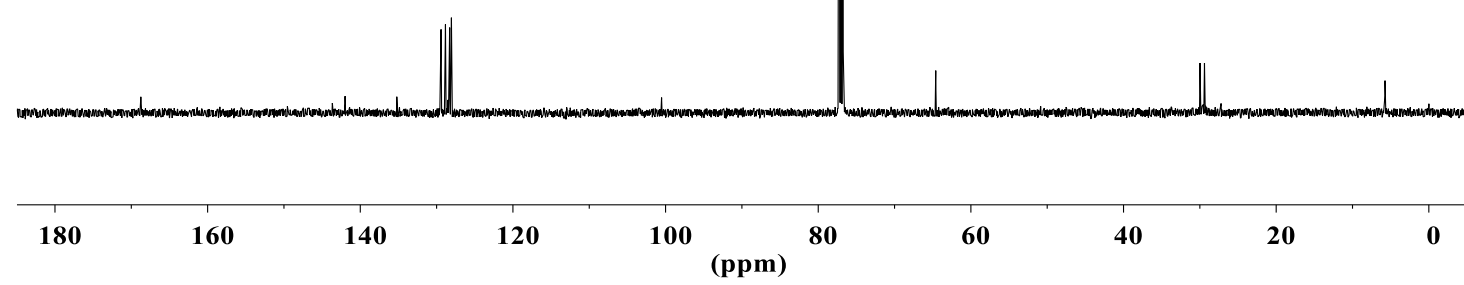

(125 $\mathrm{MHz}$ for ${ }^{13} \mathrm{C}$ NMR with $\mathrm{CDCl}_{3}$ as solvent) 


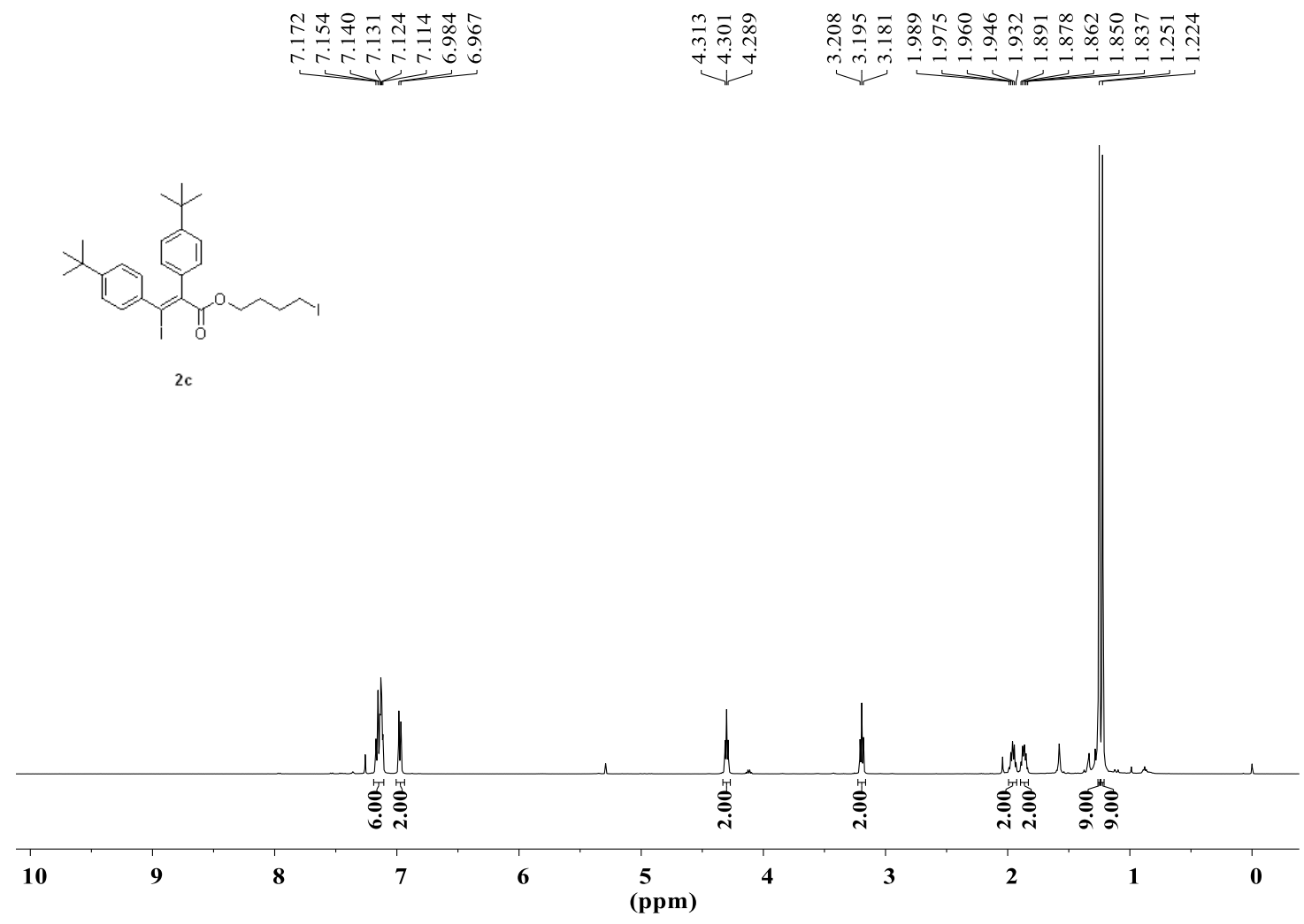

(500 MHz for ${ }^{1} \mathrm{H}$ NMR with $\mathrm{CDCl}_{3}$ as solvent)

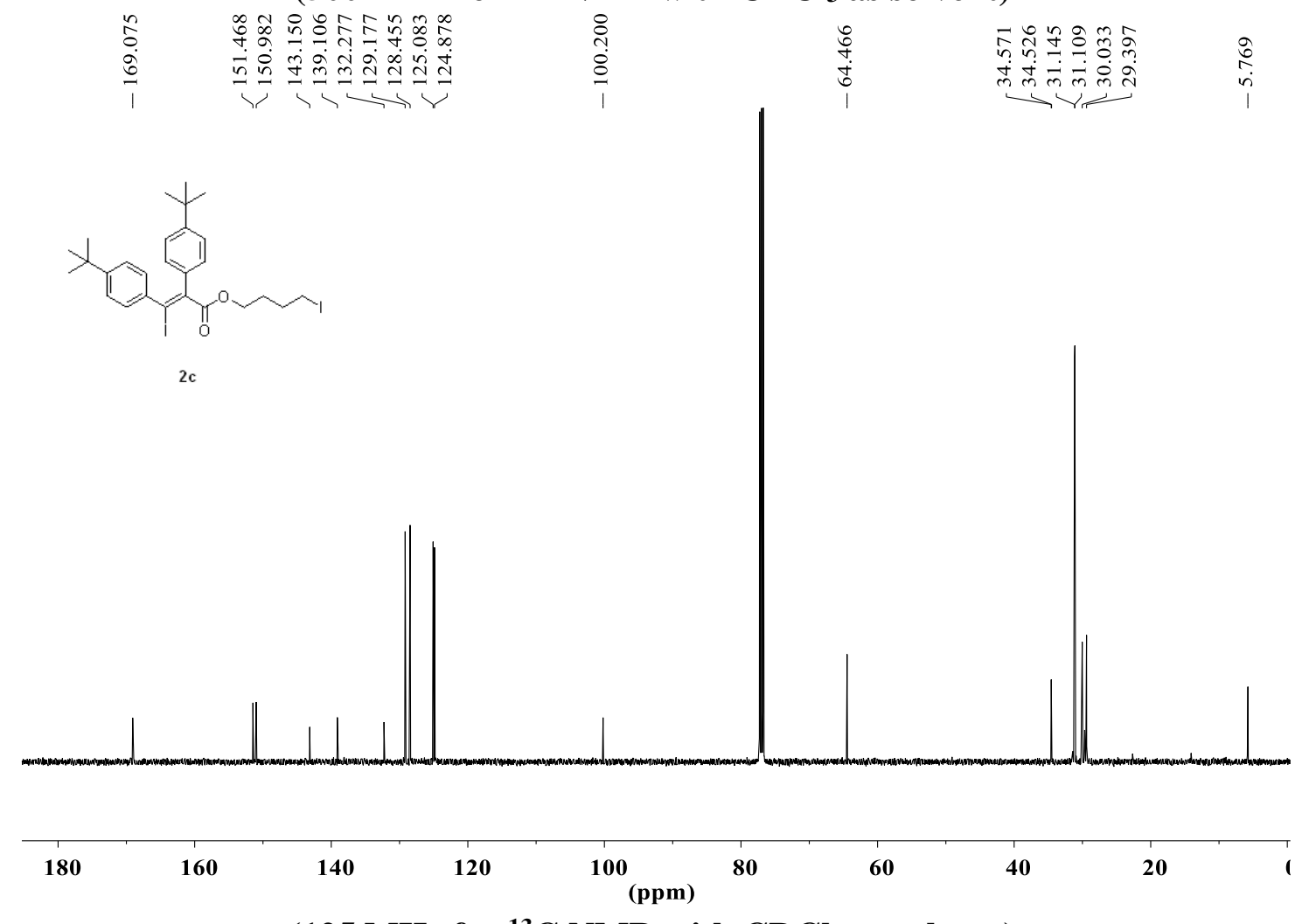

(125 MHz for ${ }^{13} \mathrm{C} \mathrm{NMR}$ with $\mathrm{CDCl}_{3}$ as solvent) 


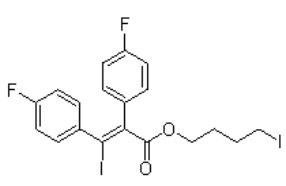

$3 c$

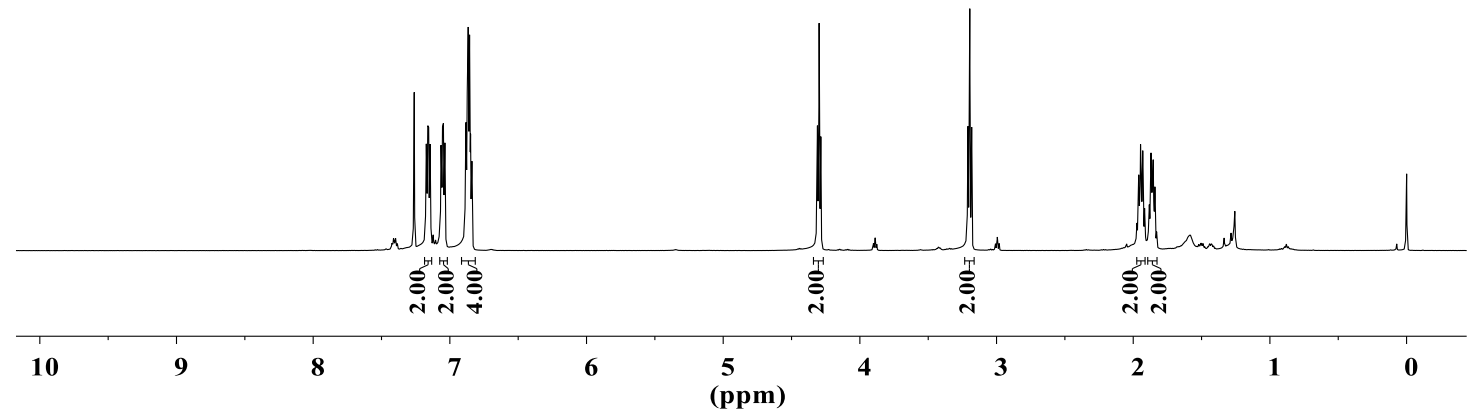

(500 MHz for ${ }^{1} \mathrm{H}$ NMR with $\mathrm{CDCl}_{3}$ as solvent)

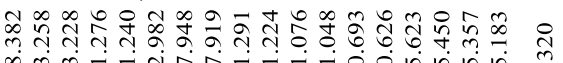

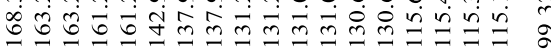
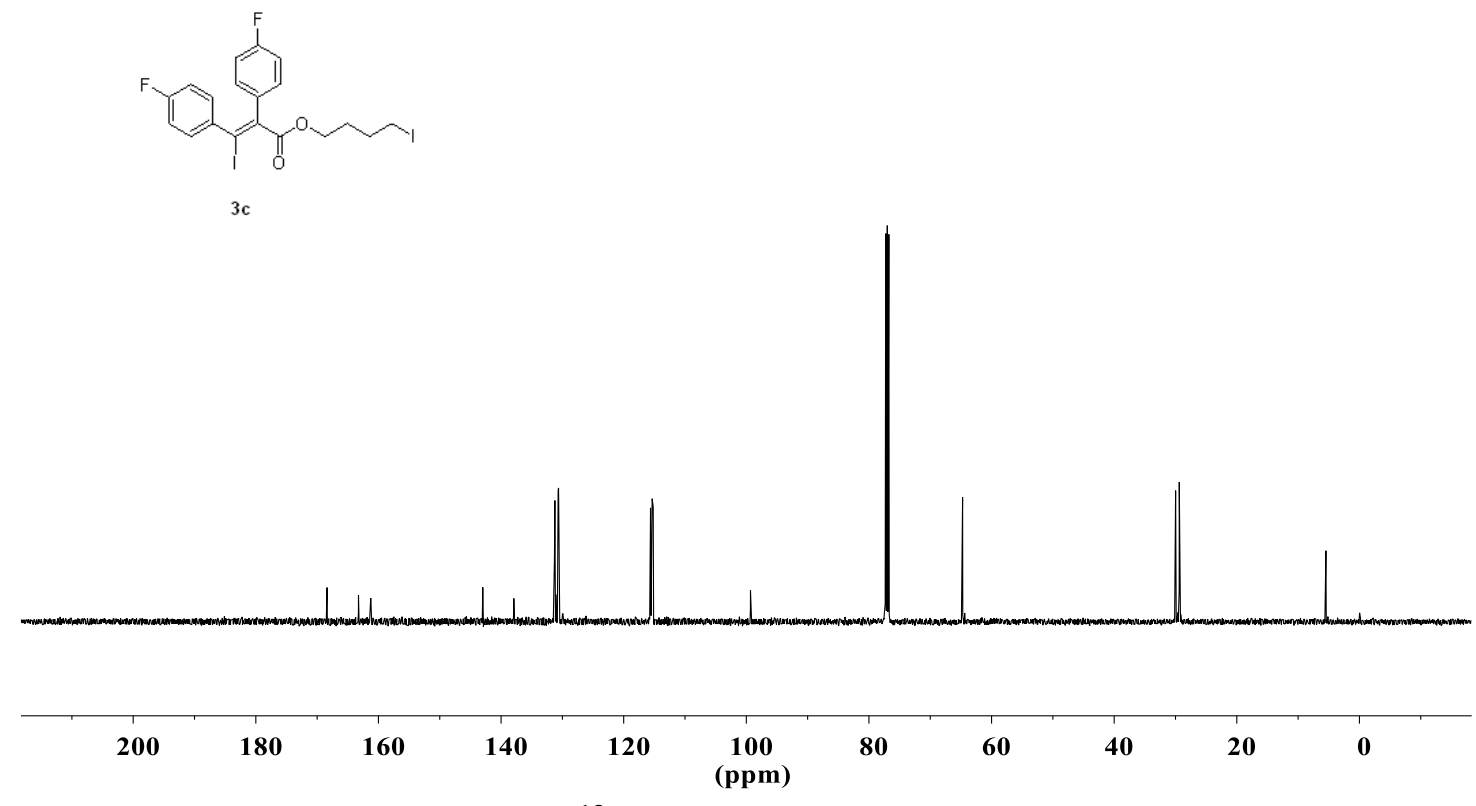

(125 $\mathrm{MHz}$ for ${ }^{13} \mathrm{C} \mathrm{NMR}$ with $\mathrm{CDCl}_{3}$ as solvent) 


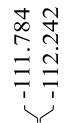

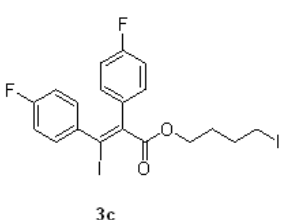

$3 c$

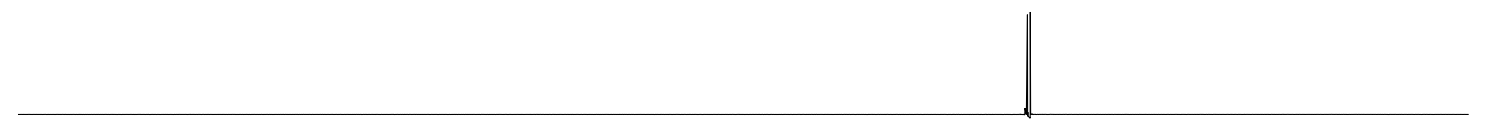

\begin{tabular}{|c|c|c|c|c|c|c|c|c|c|c|}
\hline 60 & 40 & 20 & 0 & -20 & -40 & $\begin{array}{l}-60 \\
(\mathrm{ppm})\end{array}$ & $\mathbf{- 8 0}$ & -100 & -120 & -140 \\
\hline
\end{tabular}

(470 MHz for ${ }^{19} \mathrm{~F}$ NMR with $\mathrm{CDCl}_{3}$ as solvent)
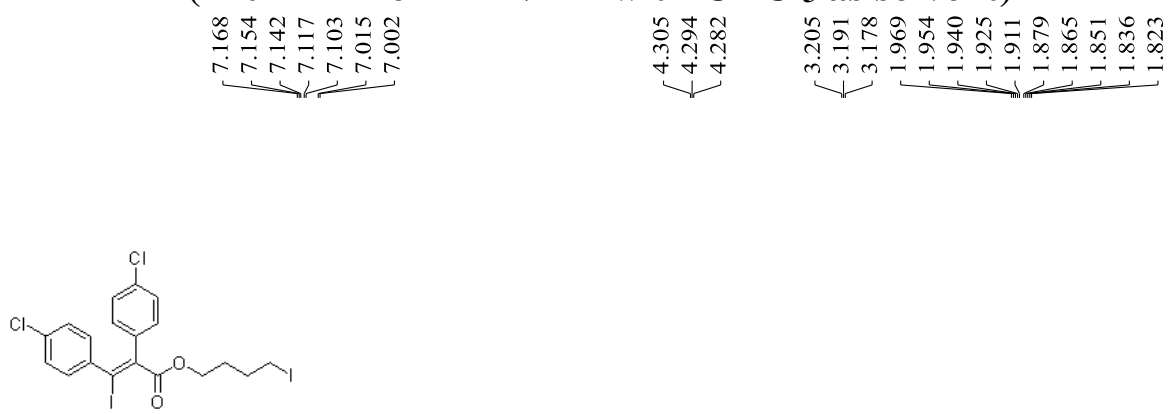

$4 \mathrm{c}$

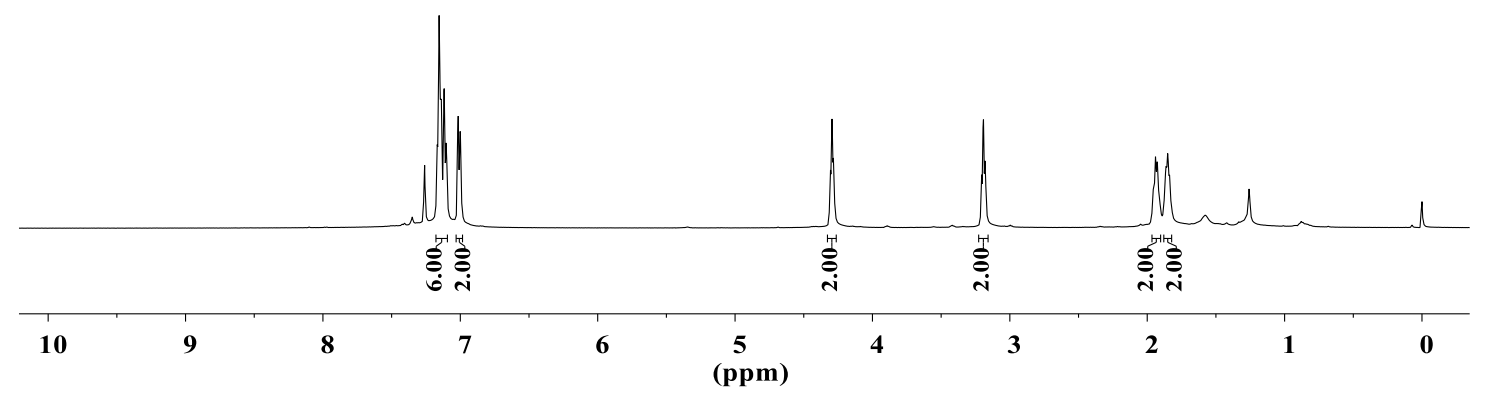

(500 MHz for ${ }^{1} \mathrm{H}$ NMR with $\mathrm{CDCl}_{3}$ as solvent) 


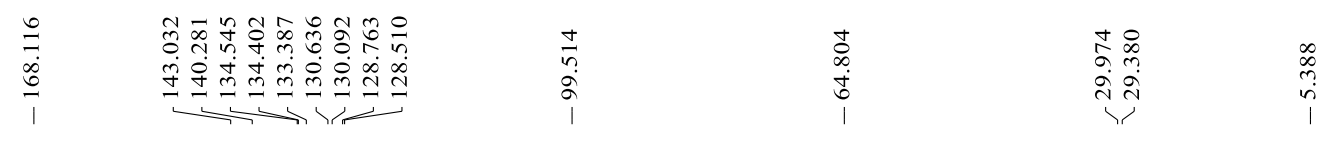

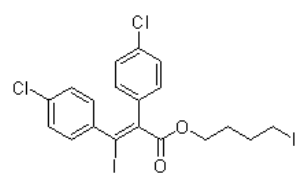

$4 c$
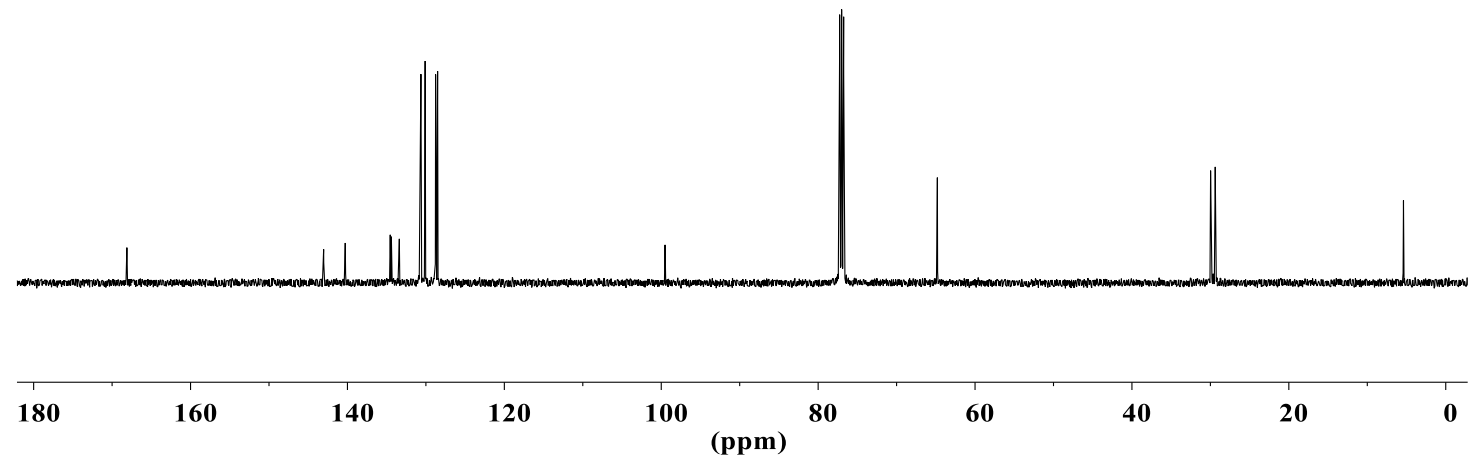

(125 $\mathrm{MHz}$ for ${ }^{13} \mathrm{C} \mathrm{NMR}$ with $\mathrm{CDCl}_{3}$ as solvent)

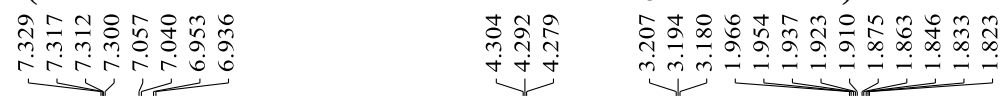

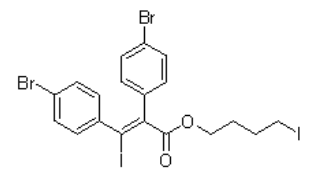

$5 c$

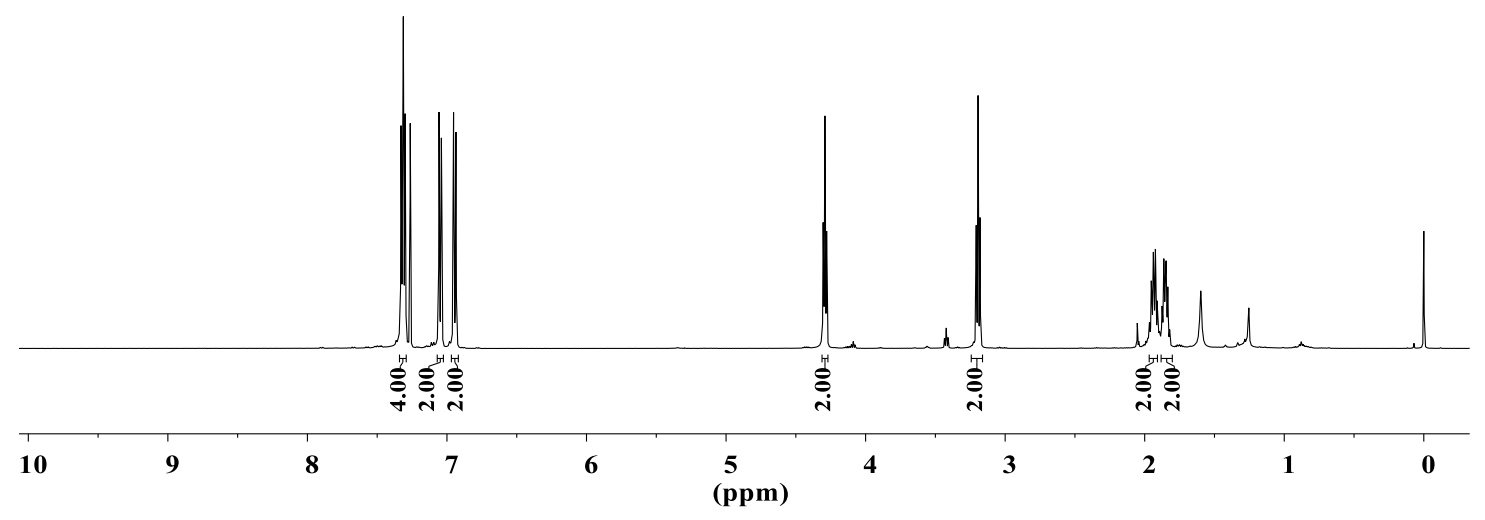

(500 MHz for ${ }^{1} \mathrm{H}$ NMR with $\mathrm{CDCl}_{3}$ as solvent) 


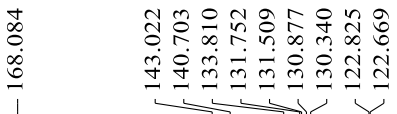

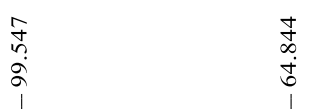

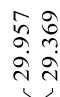

$\underset{\substack{q \\ \dot{q}}}{\dot{q}}$

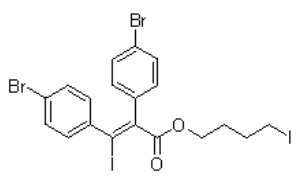

$5 c$

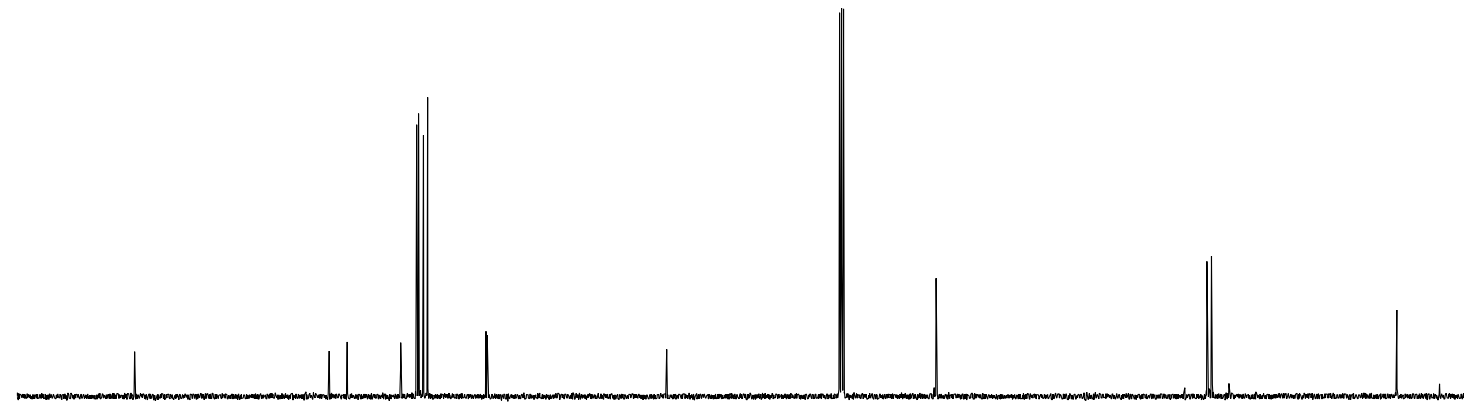

180

160

140

120

100

80

(ppm)

60

40

20

(125 MHz for ${ }^{13} \mathrm{C} \mathrm{NMR}$ with $\mathrm{CDCl}_{3}$ as solvent)

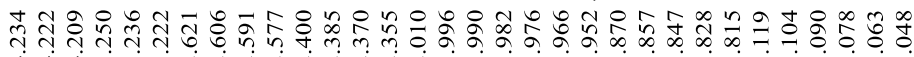

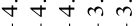

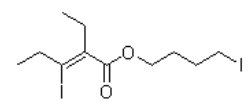

$6 c$

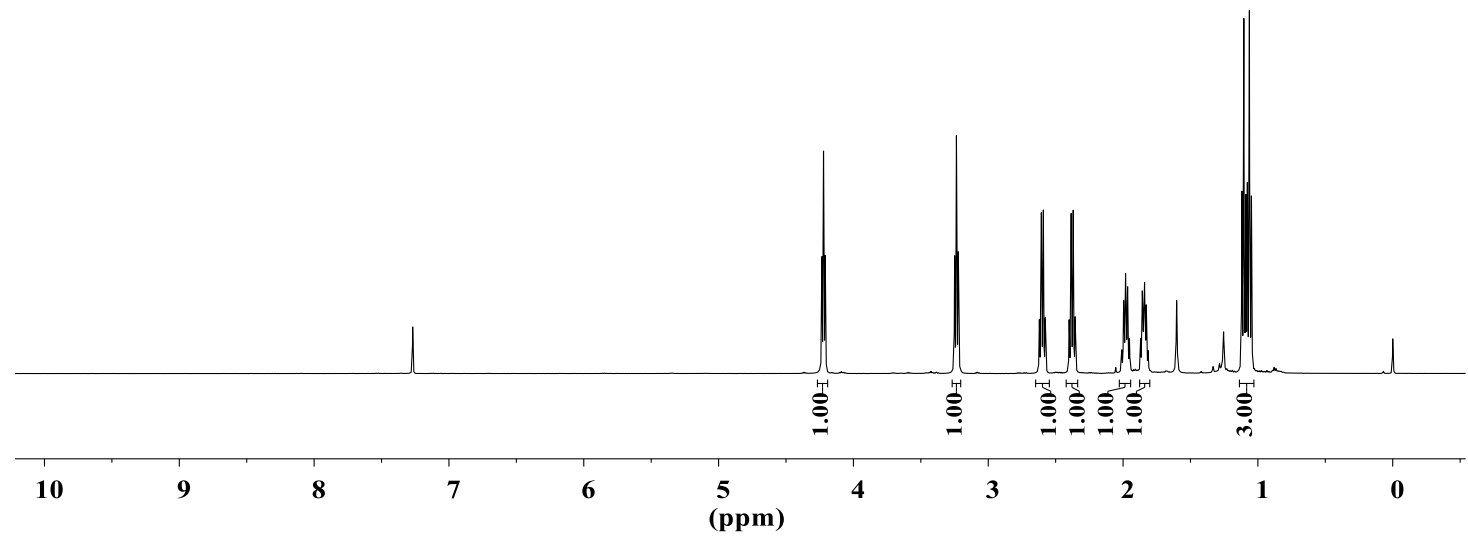

(500 MHz for ${ }^{1} \mathrm{H}$ NMR with $\mathrm{CDCl}_{3}$ as solvent) 


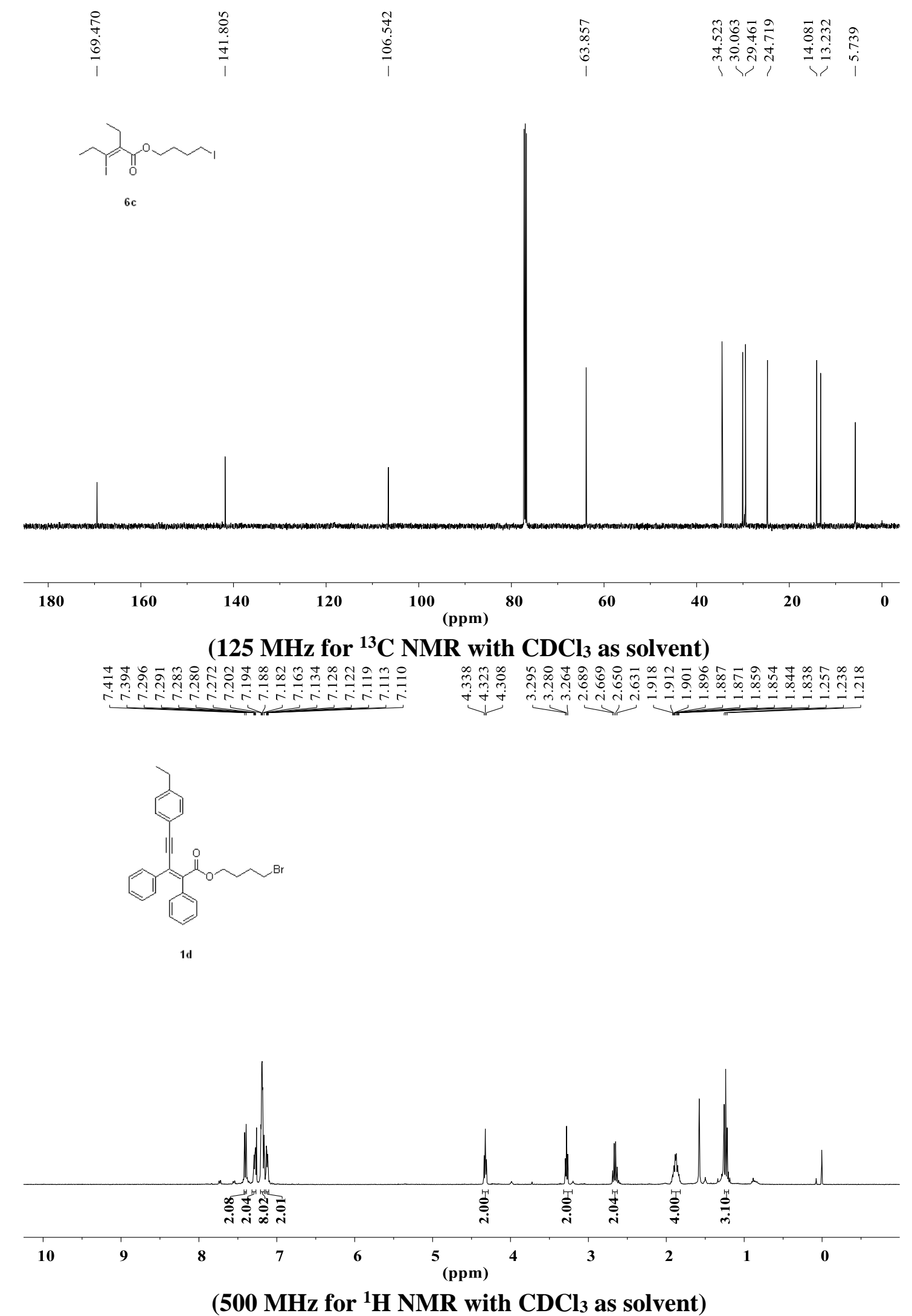




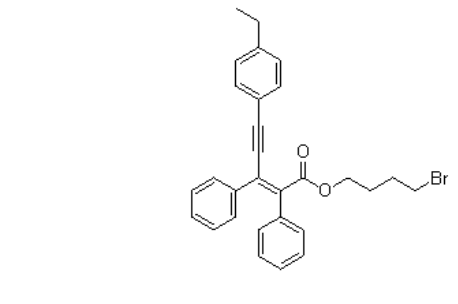

1d

\begin{tabular}{|c|c|c|}
\hline $\begin{array}{ll}\infty & \infty \\
\infty & \infty \\
\infty & \infty \\
& \infty \\
& \infty \\
1 & 1\end{array}$ & 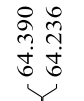 & 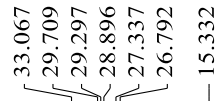 \\
\hline
\end{tabular}
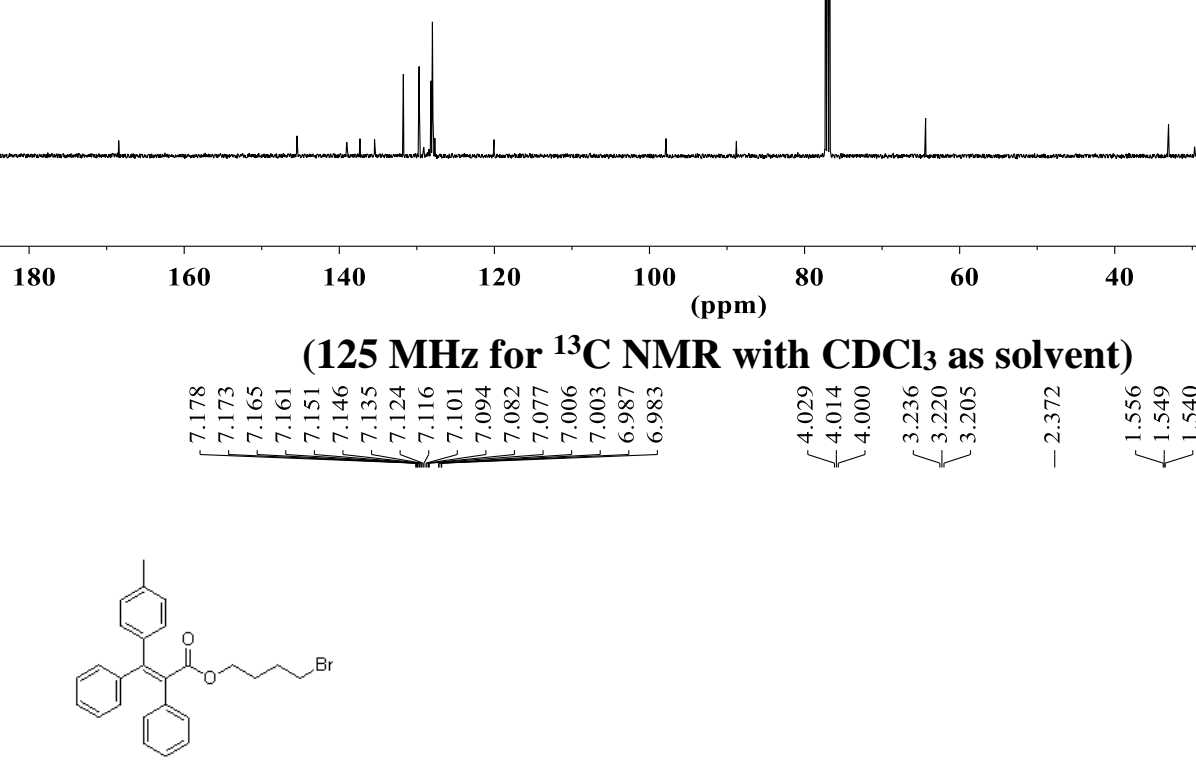

2d

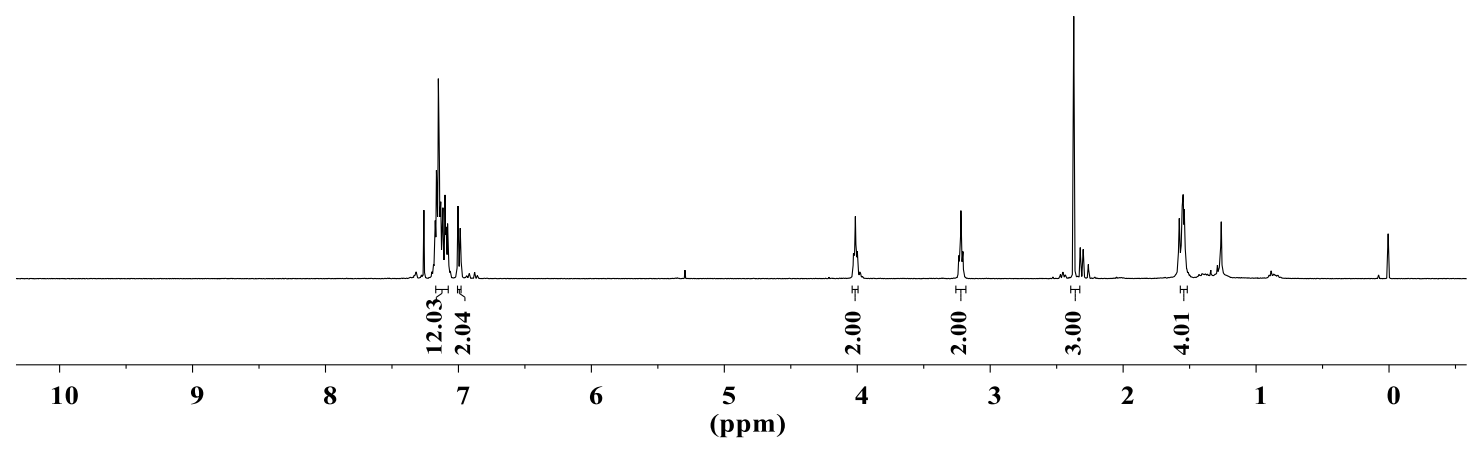

(500 MHz for ${ }^{1} \mathrm{H}$ NMR with $\mathrm{CDCl}_{3}$ as solvent) 


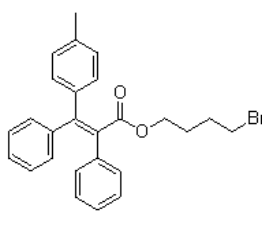

$2 d$

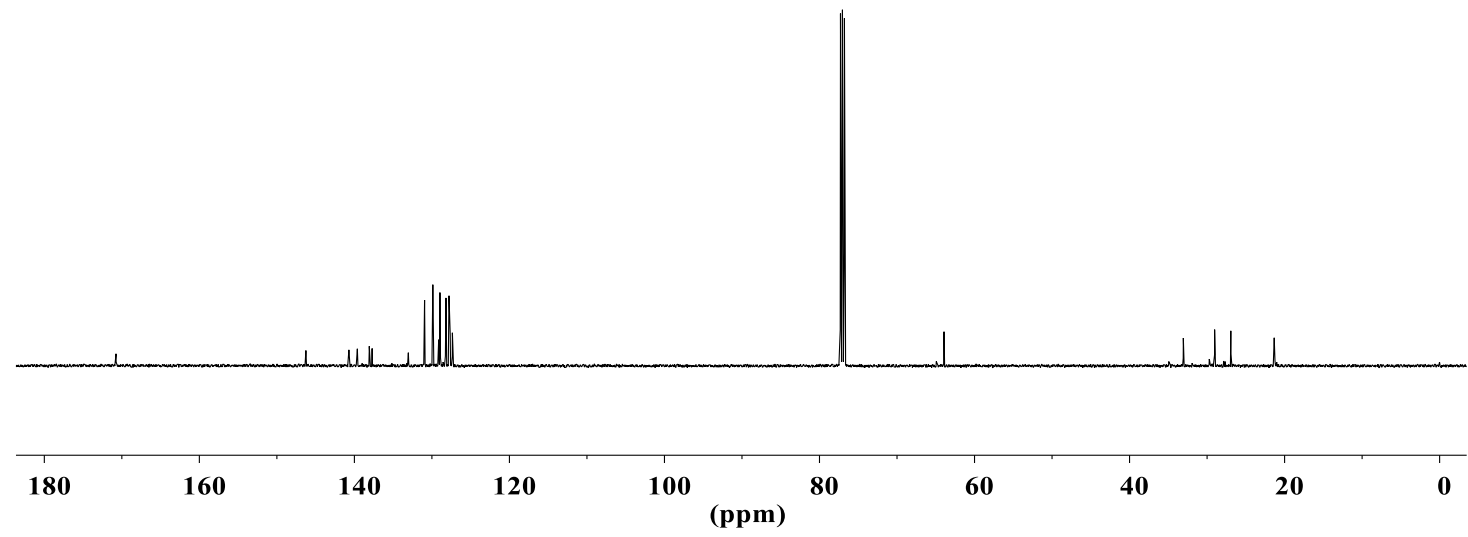

(125 MHz for ${ }^{13} \mathrm{C} \mathrm{NMR} \mathrm{with} \mathrm{CDCl}_{3}$ as solvent)

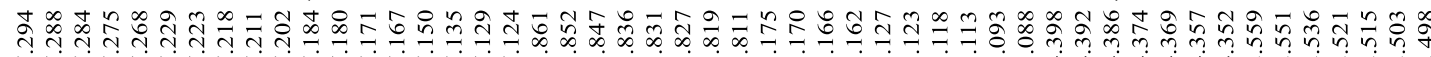

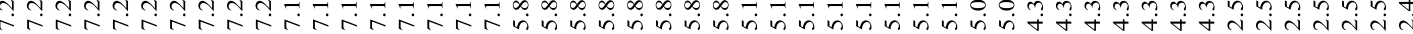
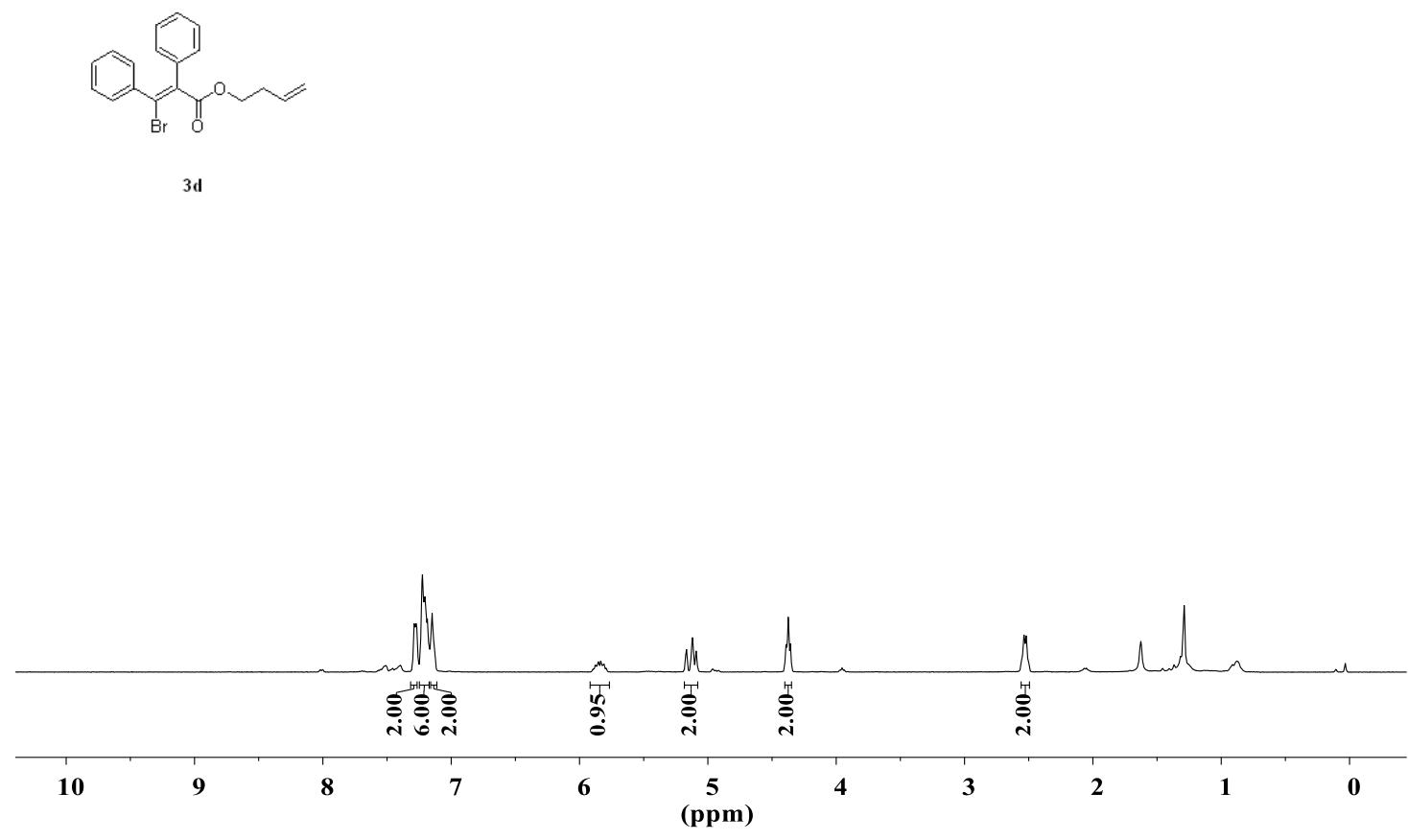

(500 MHz for ${ }^{1} \mathrm{H}$ NMR with $\mathrm{CDCl}_{3}$ as solvent) 


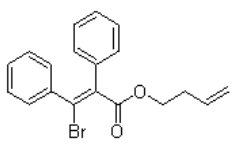

$3 d$

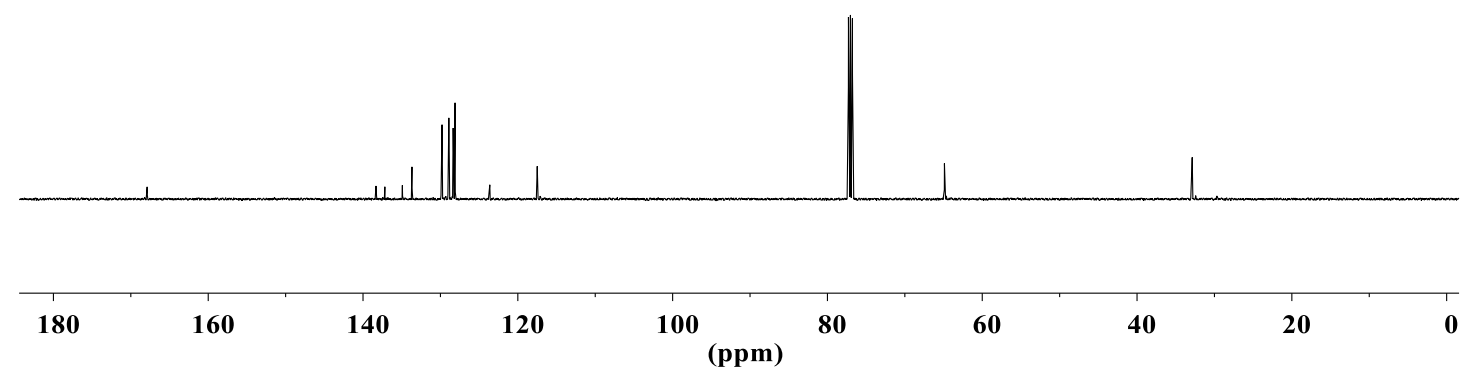

(125 $\mathrm{MHz}$ for ${ }^{13} \mathrm{C} \mathrm{NMR}$ with $\mathrm{CDCl}_{3}$ as solvent)

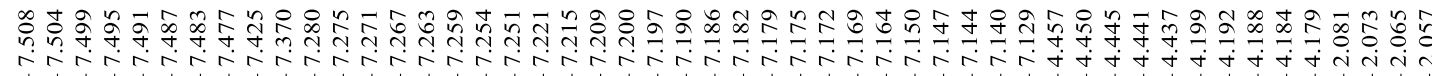

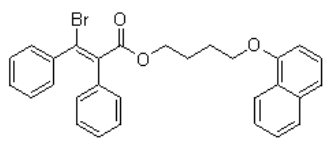

$4 d$

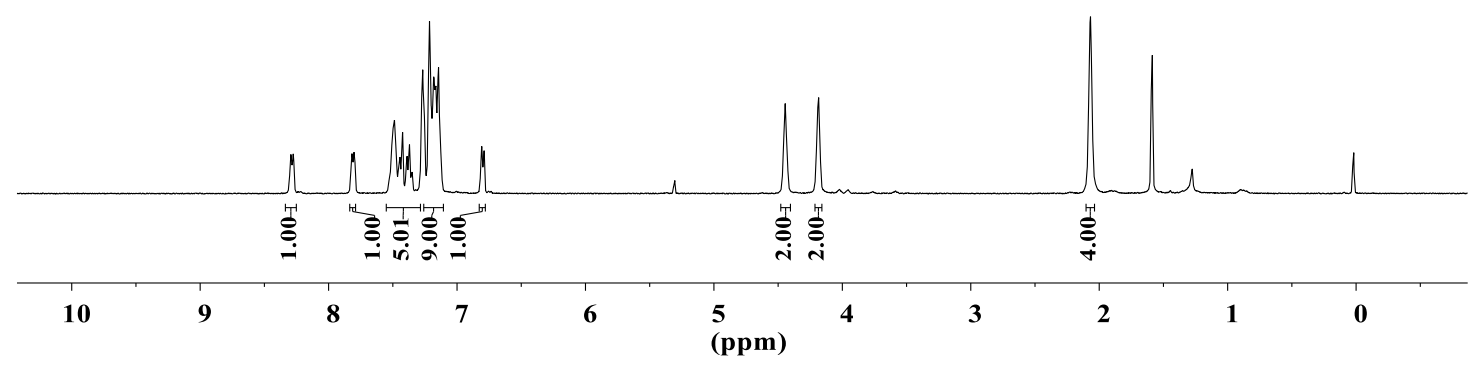

(500 MHz for ${ }^{1} \mathrm{H}$ NMR with $\mathrm{CDCl}_{3}$ as solvent) 


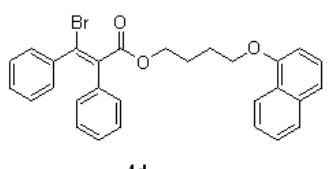

$4 d$

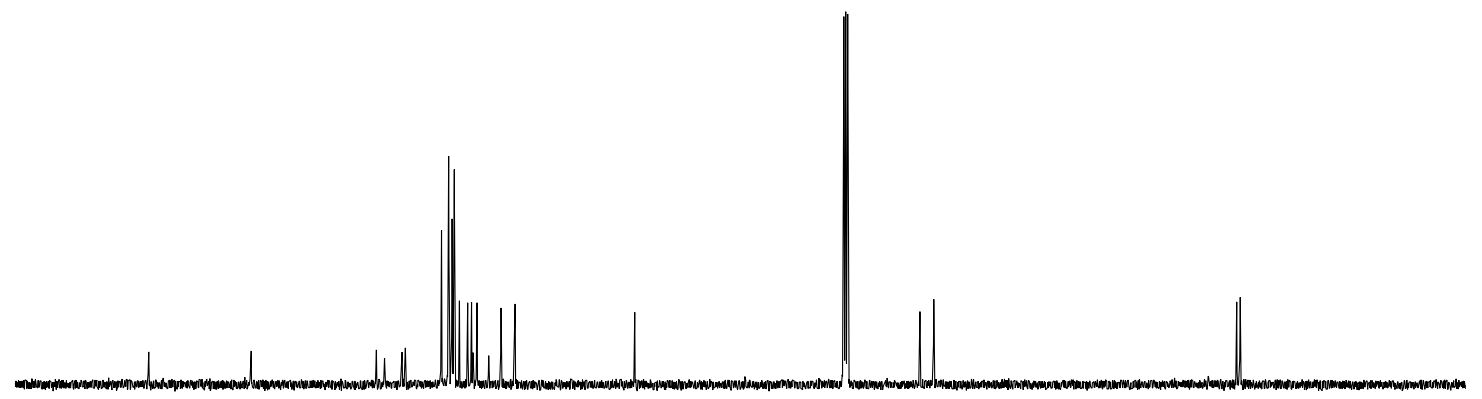

180

160

140

120

100

(ppm)

80

60

40

20

(125 MHz for ${ }^{13} \mathrm{C} \mathrm{NMR}$ with $\mathrm{CDCl}_{3}$ as solvent) 